\title{
$\Subset$ galaxies
}

\section{Radio Galaxies at}

\section{TeV Energies}




\section{Radio Galaxies at TeV Energies}





\section{Radio Galaxies at TeV Energies}

Special Issue Editor

Dorit Glawion

MDPI • Basel • Beijing • Wuhan • Barcelona • Belgrade

MDPI 
Special Issue Editor

Dorit Glawion

Erlangen Centre for Astroparticle Physics,

Friedrich-Alexander University Erlangen-Nürnberg

Germany

Editorial Office

MDPI

St. Alban-Anlage 66

4052 Basel, Switzerland

This is a reprint of articles from the Special Issue published online in the open access journal Galaxies (ISSN 2075-4434) from 2018 to 2020 (available at: https:/ /www.mdpi.com/journal/galaxies/special_ issues/RadioGalaxies).

For citation purposes, cite each article independently as indicated on the article page online and as indicated below:

LastName, A.A.; LastName, B.B.; LastName, C.C. Article Title. Journal Name Year, Article Number, Page Range.

\section{ISBN 978-3-03928-750-5 (Pbk) \\ ISBN 978-3-03928-751-2 (PDF)}

(C) 2020 by the authors. Articles in this book are Open Access and distributed under the Creative Commons Attribution (CC BY) license, which allows users to download, copy and build upon published articles, as long as the author and publisher are properly credited, which ensures maximum dissemination and a wider impact of our publications.

The book as a whole is distributed by MDPI under the terms and conditions of the Creative Commons license CC BY-NC-ND. 


\section{Contents}

About the Special Issue Editor . . . . . . . . . . . . . . . . . . . vii

\section{Dorit Glawion}

Radio Galaxies at TeV Energies: Preface

Reprinted from: galaxies 2020, 8, 18, doi:10.3390/galaxies8010018 . . . . . . . . . . . . . . 1

Frank M. Rieger and Amir Levinson

Radio Galaxies at VHE Energies

Reprinted from: galaxies 2018, 6, 116, doi:10.3390/galaxies6040116 . . . . . . . . . . . . . . 4

Kouichi Hirotani

Very High-Energy Emission from the Direct Vicinity of Rapidly Rotating Black Holes

Reprinted from: galaxies 2018, 6, 122, doi:10.3390/galaxies6040122 . . . . . . . . . . . . . . . 29

Manel Perucho

Dissipative Processes and Their Role in the Evolution of Radio Galaxies

Reprinted from: galaxies 2019, 7,70, doi:10.3390/galaxies7030070 . . . . . . . . . . . . . . . 66

Bindu Rani

Radio Galaxies-The TeV Challenge

Reprinted from: galaxies 2019, 7, 23, doi:10.3390/galaxies7010023 . . . . . . . . . . . . . . . . 101

Isak Delberth Davids, Markus Böttcher and Michael Backes

Centaurus A: Hard X-ray and High-Energy Gamma-Ray Light Curve Correlation

Reprinted from: galaxies 2019, 7, 44, doi:10.3390/galaxies7020044 . . . . . . . . . . . . . . 120

Silke Britzen, Christian Fendt, Michal Zajaček, Frédéric Jaron, Ilya Pashchenko, Margo F. Aller and Hugh D. Aller

3C 84: Observational Evidence for Precession and a Possible Relation to TeV Emission

Reprinted from: galaxies 2019, 7,72, doi:10.3390/galaxies7030072 . . . . . . . . . . . . . . . 128

Ranieri Diego Baldi, Eleonora Torresi, Giulia Migliori and Barbara Balmaverde

The High Energy View of FR0 Radio Galaxies

Reprinted from: galaxies 2019, 7,76, doi:10.3390/galaxies7030076 . . . . . . . . . . . . . . 159 



\section{About the Special Issue Editor}

Dorit Glawion is a research scientist at the Erlangen Centre for Astroparticle Physics at the Friedrich-Alexander University Erlangen-Nürnberg, Germany. Her main research interests are acceleration and emission mechanisms in high-energy astronomical objects using gamma-ray as well as multi-frequency observations. Objects in focus are supermassive black holes, active galactic nuclei, and extragalactic jets. Having been a member of the international gamma-ray observatory collaborations MAGIC and FACT before, she now is part of the H.E.S.S. collaboration. 



\title{
Radio Galaxies at TeV Energies: Preface
}

\author{
Dorit Glawion ${ }^{\dagger}$ \\ Landessternwarte, Zentrum für Astronomie, Universität Heidelberg, D-69117 Heidelberg, Germany; \\ dorit.glawion@fau.de \\ † Current Address: Erlangen Centre for Astroparticle Physics, Friedrich-Alexander-Universität \\ Erlangen-Nürnberg, D-91058 Erlangen, Germany.
}

Received: 31 January 2020; Accepted: 10 February 2020; Published: 22 February 2020

\begin{abstract}
The majority of the known extragalactic sky from $\mathrm{TeV}$ gamma-ray energies consists of blazars having plasma jets pointing in the direction of the line-of-sight, which results in a large Doppler boosting of their emission. Up to now, only six galaxies with a larger viewing angle have been detected in the $\mathrm{TeV}$ range. These objects also show fascinating properties, such as fast variability or spectral features and are called "radio galaxies". The TeV radio galaxies provide a unique laboratory for studying key aspects of active galactic nuclei. This Special Issue of Galaxies targets these exciting objects.
\end{abstract}

Keywords: active galactic nuclei; radio galaxies; emission: non-thermal; gamma-rays

\section{Introduction}

The jets of the majority of gamma-ray detected active galactic nuclei (AGN) are observed under very small angles between the jet-axis and the line-of-sight. Those objects are called blazars, an abbreviation for "blazing quasi stellar object". Due to the motion of the particles in the jet close to the speed of light, the radiation coming from blazars is strongly Doppler boosted. The frequently observed rapid variations of the brightness of these objects are related to larger, time-dilated emission regions. Instead, radio galaxies are being viewed under larger angles and the Doppler boosting of the flux is only moderate or negligible. For this reason almost all detected gamma-ray AGN are viewed under a small angle, because the amplification of the flux leads to a higher detection probability. It is also the reason why it was originally believed that blazars are the only objects that are detected in the gamma-ray band and which show flux variations. However, observations in recent years with the Fermi Large Area Telescope and imaging air Cherenkov telescopes have revealed that radio galaxies show similar variability time scales as blazars, which limit the theoretical models for particle acceleration and the emission, e.g., [1-3]. Furthermore, in case of a weak Doppler-boosted emission from an AGN jet with larger viewing angle, additional emission components may become visible.

So far, 78 individual AGN were detected by ground-based gamma-ray instruments until December 2019 in the very-high-energy (VHE) gamma-ray range between a few tens of $\mathrm{GeV}$ up to about $100 \mathrm{TeV}$. All but six of these objects are blazars. The non-blazar AGN are: Centaurus A, M87, NGC 1275, IC 310, PKS 0625-354, and 3C 264.

\section{Summary of the Contributions}

The present issue contains a general review by Rieger and Levinson with a summary of the observed characteristics of all radio galaxies in the VHE band together with a recap of state-of-the-art theoretical models for the gamma-ray emission in AGN [4]. A dedicated article by Hirotani reviews the production of VHE emission in the magnetosphere close to rotating black holes [5]. Perucho summarizes the conditions under which dissipative processes of magnetic or kinetic energy in 
relativistic jets occur and describes their role in the evolution of AGN jets and propagation [6]. Rani reviews combined radio and gamma-ray observations with special emphasis on the properties of $\mathrm{TeV}$ detected radio galaxies [7]. Such studies are crucial for the understanding of the location of gamma-ray emission sites jets of AGN in general.

Centaurus $\mathrm{A}$ is one of the best studied extragalactic objects in general and is located at a distance of $\sim 3.7 \mathrm{Mpc}$. The energy spectrum in the $\mathrm{GeV}$ to $\mathrm{TeV}$ band of Centaurus A shows an usual hardening at higher energies, indicating a new gamma-ray component connected with the high energy emission. So far, no flux variations were measured from Centaurus A in the gamma-ray band. For this issue, Davids et al. performed a correlation study of long-term light curves in the high-energy gamma-ray and X-ray band using Fermi-LAT and Swift-BAT data [8].

NGC 1275, also known as 3C 84, is the central galaxy of the Perseus cluster of galaxies. The sub-parsec radio jet shows a new component which appeared about ten years ago and keeps growing in brightness as it moves along the jet. In late 2016 to beginning of 2017, NGC 1275 showed an extremely bright and fast VHE outburst that was fifty times brighter than previously reported measurements. One research article in this issue by Britzen et al. [9] investigates high-resolution very-long-baseline-interferometry data of NGC 1275 and studies the correlation of the parsec-scale images of the jet with the flaring VHE emission behavior.

All detected non-blazar AGN show a Faranoff-Riley type I (FR I) radio morphology on kilo-parsec scales with jets ending in diffuse "edge darkened" plume-like structures. Baldi et al. examined for this issue the possibility of the detection of FR 0 radio galaxies in the VHE band, low-power radio galaxies with limited jet structures [10]. They review the results on the discovery of the FR 0 radio galaxy Tol 1326-379 in the gamma-ray band with Fermi-LAT.

This special issue contains a sample of articles that effectively summarize the main aspects of $\mathrm{TeV}$ detected radio galaxies from the observational, theoretical, as well as simulation point of view, highlights some individual objects, and discusses a possible new class of gamma-ray loud radio galaxies.

Funding: D.G. acknowledges the support through the grant 05A17VH5/BMBF/Wagner by the German Ministry for Education and Research (BMBF).

Acknowledgments: The author would like to thank all contributors to this Special Issue.

Conflicts of Interest: The author declares no conflict of interest.

\section{References}

1. Abramowski, A.; Acero, F.; Aharonian, F.; Akhperjanian, A.G.; Anton, G.; Balzer, A.; Barnacka, A.; Barres de Almeida, U.; Becherini, Y.; Becker, J.; et al. The 2010 Very High Energy $\gamma$-Ray Flare and 10 Years of Multi-wavelength Observations of M 87. Astrophys. J. 2012, 746, 151. [CrossRef]

2. Aleksić, J.; Ansoldi, S.; Antonelli, L.A.; Antoranz, P.; Babic, A.; Bangale, P.; Barrio, J.A.; González, J.B.; Bednarek, W.; Bernardini, E.; et al. Black hole lightning due to particle acceleration at subhorizon scales. Science 2014, 346, 1080-1084. [CrossRef] [PubMed]

3. MAGIC Collaboration; Ansoldi, S.; Antonelli, L.A.; Arcaro, C.; Baack, D.; Babić, A.; Banerjee, B.; Bangale, P.; Barres de Almeida, U.; Barrio, J.A.; et al. Gamma-ray flaring activity of NGC 1275 in 2016-2017 measured by MAGIC. Astron. Astrophys. 2018, 617, A91.

4. Rieger, F.M.; Levinson, A. Radio Galaxies at VHE Energies. Galaxies 2018, 6, 116. [CrossRef]

5. Hirotani, K. Very High-Energy Emission from the Direct Vicinity of Rapidly Rotating Black Holes. Galaxies 2018, 6, 122. [CrossRef]

6. Perucho, M. Dissipative processes and their role in the evolution of radio galaxies. Galaxies 2019, 7, 70. [CrossRef]

7. Rani, B. Radio Galaxies-The TeV Challenge. Galaxies 2019, 7, 23. [CrossRef]

8. Davids, I.D.; Böttcher, M.; Backes, M. Centaurus A: Hard X-ray and high-energy gamma-ray light curve correlation. Galaxies 2019, 7, 44. [CrossRef] 
9. Britzen, S.; Fendt, C.; Zajaček, M.; Jaron, F.; Pashchenko, I.; Aller, M.F.; Aller, H.D. 3C 84: Observational Evidence for Precession and a Possible Relation to TeV Emission. Galaxies 2019, 7, 72. [CrossRef]

10. Baldi, R.D.; Torresi, E.; Migliori, G.; Balmaverde, B. The High Energy View of FR0 Radio Galaxies. Galaxies 2019, 7, 76. [CrossRef]

(c) 2020 by the authors. Licensee MDPI, Basel, Switzerland. This article is an open access article distributed under the terms and conditions of the Creative Commons Attribution (CC BY) license (http://creativecommons.org/licenses/by/4.0/). 


\title{
Radio Galaxies at VHE Energies
}

\author{
Frank M. Rieger ${ }^{1, *}$ and Amir Levinson ${ }^{2,3}$ \\ 1 ZAH, Institut für Theoretische Astrophysik, Heidelberg University, Philosophenweg 12, \\ 69120 Heidelberg, Germany \\ 2 The Raymond and Beverly Sackler School of Physics and Astronomy, Tel Aviv University, \\ Tel Aviv 69978, Israel; Levinson@tauex.tau.ac.il \\ 3 Yukawa Institute for Theoretical Physics, Kyoto University, Oiwake-cho, Kitashirakawa, Sakyo-ku, \\ Kyoto 606-8502, Japan \\ * Correspondence: f.rieger@uni-heidelberg.de
}

Received: 9 October 2018; Accepted: 8 November 2018; Published: 15 November 2018

\begin{abstract}
Radio Galaxies have by now emerged as a new $\gamma$-ray emitting source class on the extragalactic sky. Given their remarkable observed characteristics, such as unusual gamma-ray spectra or ultrafast VHE variability, they represent unique examples to probe the nature and physics of active galactic nuclei (AGN) in general. This review provides a compact summary of their observed characteristics at very high $\gamma$-ray energies (VHE; greater than $100 \mathrm{GeV}$ ) along with a discussion of their possible physics implications. A particular focus is given to a concise overview of fundamental concepts concerning the origin of variable VHE emission, including recent developments in black hole gap physics.
\end{abstract}

Keywords: gamma-rays; radio galaxies; emission: non-thermal; origin: jet; origin: black hole

\section{Introduction}

The current decade has seen a tremendous progress in the extragalactic Gamma-Ray Astronomy. Numerous new sources have been discovered by the current generation of instruments, sometimes with highly unexpected and extreme characteristics. More than 2900 of the identified or associated high energy (HE, greater than $100 \mathrm{MeV}$ ) sources in the Fermi-LAT eight-year Point Source List (FL8Y) ${ }^{1}$, are active galactic nuclei (AGN) of the blazar class. In the very high energy (VHE, greater than $100 \mathrm{GeV}$ ) domain the detection of about $70 \mathrm{AGN}$ is currently summarised in the TeVcat catalog ${ }^{2}$. Again, most of these sources are of the blazar type, i.e., radio-loud AGN such as BL Lac objects where the jet is thought to be inclined at very small viewing angles $i$ to the line of sight. This results in substantial Doppler-boosting of their intrinsic jet emission, $S(v)=D^{a} S^{\prime}\left(v^{\prime}\right)$ where $D=1 /\left[\Gamma_{b}\left(1-\beta_{b} \cos i\right)\right]$ denotes the Doppler factor and $\Gamma_{b}=\left(1-v_{b}^{2} / c^{2}\right)^{-1 / 2}$ the jet bulk Lorentz factor and typically $a \geq 2$, privileging their detection on the sky. Nevertheless, non-blazar AGN such as Radio Galaxies (RGs), while less occurrent, have in the meantime solidly emerged as a new gamma-ray emitting source class as well. With their jets misaligned and associated Doppler boosting effects modest, they enable unique insights into often hidden regions and processes. This review aims at a compact summary of their properties and highlights their role in facilitating theoretical progress in AGN physics.

The unification model of radio-loud AGNs postulates that RGs are viewed at a substantial inclination $i$ to the jet axis so that the broad-line optical emitting regions become obscured by a dusty component ("torus" or warped disk) in Narrow Line RGs (NLRGs) such as in Cen A or M87 [1,2].

\footnotetext{
http://fermi.gsfc.nasa.gov/ssc/data/access/lat/fl8y/.

http://tevcat.uchicago.edu.
} 
Depending on their radio structure, RGs have early on been divided into Fanaroff-Riley I and II sources (FR I, FR II) [3], the former one (FR I) encompassing lower radio luminosity, edge-darkened sources and the latter one (FR II) higher luminous, edge-brightened sources where the radio lobes are dominated by bright hot spots. Various considerations suggest that the high-power FR II sources might be accreting in a "standard" (geometrically thin, optically thick) mode, while most FR I sources are probably supported by a radiatively inefficient accretion flow (RIAF) [4,5].

The general relationship between the blazar and RG class is complex. Urry \& Padovani (1995) have described BL Lacs as beamed FR I RGs [6], though evidence exists that the parent population of BL Lac objects contains both FR I and FR II sources $[7,8]$. A more detailed view might be to posit that X-ray loud BL Lacs (mostly HBLs, peaking in UV / X-rays) are preferentially associated with FR I, while radio-loud BL Lacs (mostly LBL, peaking in the infrared) could show a mixture of FR I and FR II morphologies [9].

\section{Radio Galaxies as VHE Emitters-Experimental Status}

In the HE range Fermi-LAT has detected about 20 RGs e.g., [10]. Six of them are also known as VHE emitters (see Figure 1), including M87 $(d \sim 16 \mathrm{Mpc}$ ), the first extragalactic source detected at VHE energies, and Cen $\mathrm{A}$, the nearest $(d \sim 4 \mathrm{Mpc}) \mathrm{AGN}$ to us.

\begin{tabular}{|c|c|c|c|c|}
\hline Name & Cross-ID & Type & Distance & $\mathrm{BH}$ mass $\left[10^{8} \mathrm{M}_{\text {sun }}\right]$ \\
\hline Cen A & NGC 5I28, & FR I & 3.7 Mpc & $(0.5-1)$ \\
\hline M87 & NGC 4486, Virgo A & FR I & $16 \mathrm{Mpc}$ & $(20-60)$ \\
\hline NGC 1275 & $3 \mathrm{C} 84$, Perseus A & FR I & $70 \mathrm{Mpc}$ & $3-4$ \\
\hline IC 310 & В03।3+4II & FR I/BL Lac & $80 \mathrm{Mpc}$ & $3[0.3 ?]$ \\
\hline $3 C 264$ & NGC 3862 & FR I & $95 \mathrm{Mpc}$ & $4-5$ \\
\hline PKS 0625-35 & $\mathrm{OH} 342$ & FR I/BL Lac & $220 \mathrm{Mpc}$ & $\sim 10$ \\
\hline
\end{tabular}

Figure 1. Radio galaxies reported at VHE energies, including estimates for their black hole masses. Cross-IDs give their alternative source identifications. Two sources, IC 310 and PKS 0625-35, may be of a transitional type.

This emergence of RGs as a new VHE emitting source class is particularly interesting. Given the substantial misalignment of their jets $\left(i>10^{\circ}\right)$, RGs are commonly thought to be characterized by rather modest Doppler boosting only (bulk Doppler factor $D \leq$ a few). If, following simple unification considerations, the nuclear emission of FR I type RGs is interpreted as "misaligned BL Lac type" (i.e., of a jet-related, homogeneous synchrotron self-Compton (SSC) origin, yet with small Doppler factor) [11], only a few sources should become detectable at $\mathrm{GeV}$ energies (which seems indeed to be the case), and almost none at TeV energies. The discovery of RGs as a new VHE emitting source class thus points to a more complex situation, and promises new insights into some of the fundamental (and often hidden) non-thermal processes in $\gamma$-ray emitting AGN. The following aims to provide a short summary of the experimental source characteristics: 


\subsection{PKS 0625-354}

The detection of VHE emission from PKS 0625-354 $(z=0.055)$ above $200 \mathrm{GeV}$ (at a level of $\sim 6 \sigma$ in $5.5 \mathrm{~h}$ of data) has been recently reported by H.E.S.S. [12]. No significant variability is found in the data. The VHE spectrum extends up to $\sim 2 \mathrm{TeV}$ and is compatible with a rather steep power law of photon index $\Gamma_{\gamma} \sim-2.8 \pm 0.5$. The VHE power is moderate with an apparent isotropic luminosity of the order of $L_{\mathrm{VHE}} \sim 5 \times 10^{42} \mathrm{erg} / \mathrm{s}$. Both leptonic and hadronic SED interpretations seem possible [12].

PKS 0625-354 is thought to harbour a black hole of mass $M_{\mathrm{BH}} \sim 10^{9} M_{\odot}$ [13] that is probably accreting in an inefficient mode. The source is known as a low excitation line radio-loud AGN, but being a transitional FR I/BL Lac object its proper classification has been debated. Recent findings are favouring its classification as a BL Lac object with non-modest Doppler boosting [14-16]. It inclusion in the list of (misaligned) "radio galaxies" may thus have to be re-considered, limiting possible inferences as to the physical origin of its non-thermal emission based on current data e.g., [12,17].

\section{2. $3 C 264$}

The most recent addition to the RG list has been the FR I source 3C 264 ( $d \sim 95 \mathrm{Mpc})$ seen by VERITAS (with a significance level of $\sim 5.4 \sigma$ in $12 \mathrm{~h}$ of data) [18]. Given an estimated black hole mass $M_{\mathrm{BH}} \sim 5 \times 10^{8} M_{\odot}[19]$, the VHE luminosity appears to be moderate ( $\sim 1 \%$ of the Crab Nebula) with an isotropic equivalent $L(>300 \mathrm{GeV}) \sim 10^{42} \mathrm{erg} / \mathrm{s}$. 3C 264 has been included in the 3FHL catalog, that lists Fermi-LAT sources which are significantly detected above $10 \mathrm{GeV}$ [20]. The reported VHE flux level seems roughly compatible with a simple power law extrapolation based on the 3FHL results (FHL photon index of $-1.65 \pm 0.33$ ). There are indications, though, that the VHE spectrum is relatively hard (when compared to other VHE sources) with a photon index close to $\Gamma_{\gamma} \sim-2.3$. The source shows a low, weakly variable VHE flux along with some month-scale variations. While 3C 264 is known for rapidly evolving knot structures in its jet up to some hundred of parsecs [21], no major knot activity has been observed around the time of the VERITAS observations. Given the previously noted unclear classification of PKS 0625-354, 3C 264 may be the most distant RG detected at VHE so far.

\subsection{NGC 1275}

NGC 1275 (3C 84), the central Perseus cluster RG at a distance of $\sim 70 \mathrm{Mpc}$, has been detected at VHE energies above $100 \mathrm{GeV}$ by MAGIC, initially (based on data between 2009-2011) at moderate flux levels ( $\sim 3 \%$ of the Crab Nebula) and with a very steep VHE spectrum (photon index of $\Gamma_{\gamma} \sim-4.1$ if characterized by a power law) extending up to $\sim 650 \mathrm{GeV}[22,23]$. When HE (Fermi-LAT) and VHE data are combined, the average ("quiescent") $\gamma$-ray spectrum appears compatible with either a log-parabola or a power-law with a sub-exponential cut-off, suggestive of a common physical origin and of a peak or cut-off around several GeV. More recently, MAGIC has reported the detection of strong VHE activity with flux levels increased by up to a factor of 50 around 31 December 2016 and 1 January 2017 (reaching $\sim 1.5$ of the Crab Nebula or an isotropic equivalent $L_{V H E} \sim 10^{45} \mathrm{erg} / \mathrm{s}$ ) [24].

Significant day-scale variability has been observed, with the flux doubling timescales as short as $\Delta t_{\text {obs }} \sim 10$ h. The VHE SED measured up to $>1 \mathrm{TeV}$ shows a curved shape (cf. Figure 2), compatible with an exponential cut-off around a few hundred GeV. The possibility of a joined HE-VHE fit along with day-scale variability, suggests that the HE-VHE emission originates in a (possibly, single) compact zone. The physical nature of this emission is not yet clear, though magnetospheric processes have been favoured over mini-jets- and jet-cloud-interaction scenarios [24].

The central engine in NGC 1275 hosts a black hole of mass $M_{\mathrm{BH}} \sim(3-4) \times 10^{8} M_{\odot}[25,26]$ and exhibits a pc-scale radio jet orientated at $i \sim 30-60^{\circ}[27,28]$. Its inferred jet power is of the order of $L_{j} \sim(0.5-1) \times 10^{44} \mathrm{erg} / \mathrm{s}[26,29]$. The high ratio $L_{V H E} / L_{j} \sim 10$ thus raises questions for a magnetospheric origin of the gamma-ray flare emission, cf. [30] (see also below), unless strong short-term magnetic flux increase would occur. On the other hand, a homogeneous SSC interpretation, assuming the sub-pc scale jet to be weakly misaligned $\left(i \lesssim 20^{\circ}\right)$, would be in tension with the inferred 
jet inclination on pc-scales. This could perhaps be alleviated if the emitting component would, for example, follow a non-straight trajectory that relaxes with distances, or if the jet has some internal structure (e.g., spine-shear) allowing for multiple contributions and a more complex inverse Compton interplay [31]. Opacity constraints may pose a severe problem, though (see below). At the moment detailed modelling seems required before firm conclusions can be drawn.

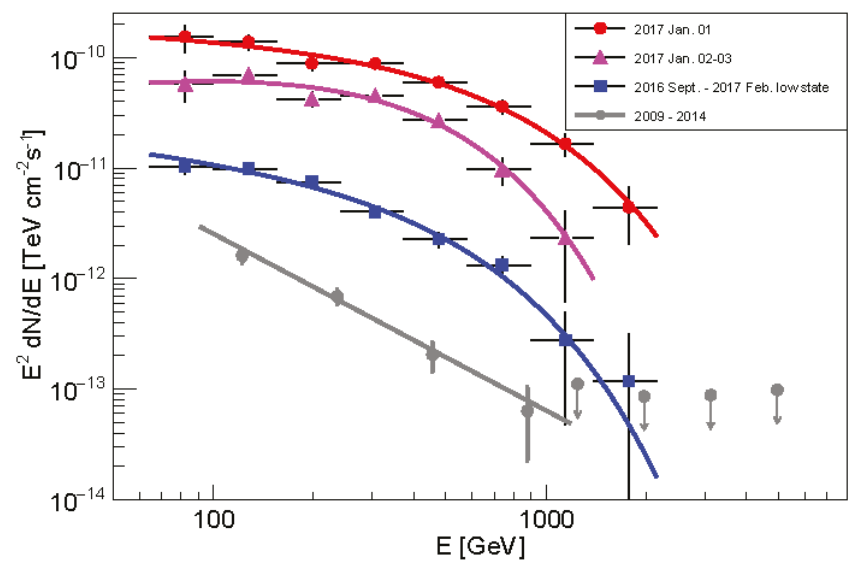

Figure 2. The VHE spectral energy distributions (SEDs) of NGC 1275 as measured by MAGIC during different periods. Significant curvature is evident, suggestive of an exponential cut-off around a few hundred GeV. For comparison the averaged spectrum based on observations in 2009 to 2014 is shown in grey. From Ref. [24].

\subsection{Centaurus $A$}

As the nearest $\mathrm{AGN}(d \simeq 3.7 \mathrm{Mpc})$ Centaurus $\mathrm{A}(\mathrm{Cen} A)$ belongs to the best studied extragalactic sources. Its central engine hosts a black hole of mass $(0.5-1) \times 10^{8} M_{\odot}$ e.g., [32] and emits (assuming a quasar-type SED) a bolometric luminosity of $L_{\mathrm{bol}} \sim 10^{43} \mathrm{erg} / \mathrm{s}$ [33]. This is much less than the expected Eddington luminosity $L_{E d d}$ and suggests that accretion in its inner part might occur in a radiatively inefficient mode [34,35]. At radio frequencies, Cen A has revealed a peculiar morphology including a compact radio core, a sub-pc scale jet and counter-jet, a one-sided kpc-scale jet and inner lobes, up to giant outer lobes with a length of hundreds of kiloparsec. VLBI observations indicate that Cen A is a "non-blazar" source with its inner jet misaligned by $i \sim(12-45)^{\circ}$ based on TANAMI jet-counter jet flux ratio measurements, and characterized by moderate bulk flow speeds in the radio band of $u_{j}<0.5$ c only e.g., [36].

At VHE energies Cen A has been the second RG detected by H.E.S.S. [37]. A recent, updated analysis based on more than $200 \mathrm{~h}$ of data shows that the VHE emission extends from $250 \mathrm{GeV}$ up to $\sim 6 \mathrm{TeV}$ and is compatible with a single, rather hard power-law of photon index $\Gamma_{\gamma} \simeq-2.5 \pm 0.1$ [38]. The source is relatively weak with an equivalent apparent isotropic luminosity of $L(>250 \mathrm{GeV}) \simeq(1-2) \times 10^{39} \mathrm{erg} / \mathrm{s}$. No significant VHE variability has been found, neither on monthly or yearly timescales, so that an extended origin or contribution (i.e., within the angular resolution $\sim 0.1^{\circ}$ of H.E.S.S., corresponding to $\sim 5 \mathrm{kpc}$ ) of the VHE emission cannot per se be discarded.

At $\mathrm{HE}$ energies, both the core region (i.e., within $\sim 0.1^{\circ}$ ) and the giant lobes of Cen $\mathrm{A}$ have been detected by Fermi-LAT [39-42]. Results concerning the latter indicate that HE lobe emission substantially extends beyond the radio maps. The HE emission of the lobes (most likely due to leptonic IC-CMB and IC-EBL, possibly with some additional hadronic pp) is of a particular interest as it provides model-independent information about the spatial distribution of the non-thermal electrons. Fermi-LAT has by now reported extended HE emission from only two RGs, Cen A and Fornax A ( $d \sim 20 \mathrm{Mpc})$ [43]. The core region of Cen A, on the other hand, was initially detected up to $10 \mathrm{GeV}$ (at a level of $4 \sigma$ ) based 
on ten months of data, with the HE spectrum at that time seemingly compatible with a single power law with photon index $\Gamma_{p}=-2.67 \pm 0.1$. While this HE power index is very close to the VHE one, a simple extrapolation of the HE power-law was soon found to under-predict the fluxes measured at $\mathrm{TeV}$ energies. The comparison was based on non-simultaneous HE and VHE data, but the absence of variability in both energy bands suggested that the discrepancy might be real. Refined analyses based on larger data sets have in the meantime found intriguing evidence for an unusual spectral hardening of the core spectrum by $\Delta \Gamma \sim 0.5$ around a few GeV [44,45]. The most recent analysis, involving contemporary VHE and HE data, finds (at a level of $4 \sigma$ ) that the HE spectral index changes around $E_{b} \simeq 2.8 \mathrm{GeV}$ from $\Gamma_{\gamma} \simeq-2.7$ (below $E_{b}$ ) to about $\simeq-2.3$ (above $E_{b}$ ), respectively [38], see Figure 3.

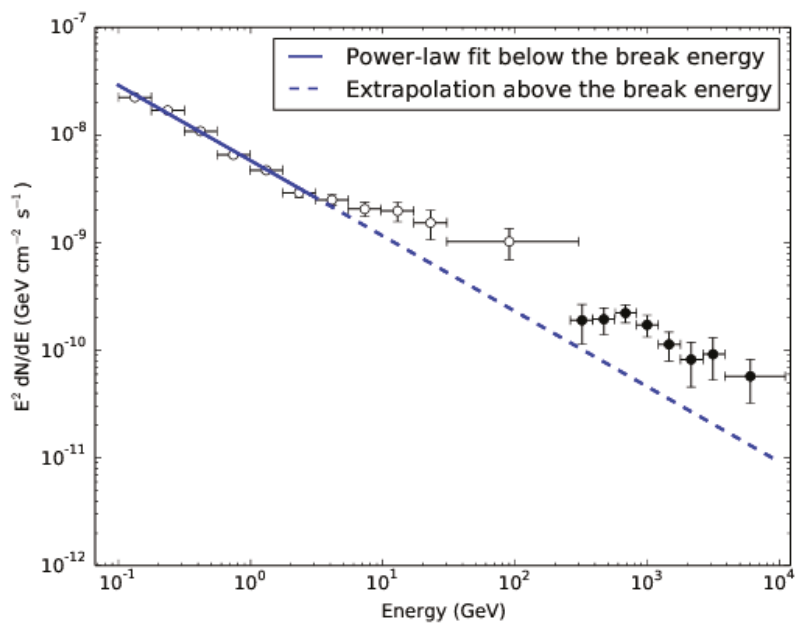

Figure 3. The gamma-ray core spectrum of Cen A above $100 \mathrm{MeV}$ based on 8 year of Fermi-LAT and more than 200 h of H.E.S.S. data. The spectrum shows an unusual spectral hardening at $E_{b} \simeq 2.8 \mathrm{GeV}$, with photon index changing by $\sim 0.4 \pm 0.1$ (assuming a broken power law), see Ref. [38] for details. This spectral feature is most naturally attributed to a second emission component that emerges towards highest energies and that allows to smoothly connect the HE emission (above $E_{b}$ ) with the VHE one.

For AGN spectral steepening at gamma-ray energies is a familiar feature that can be related to classical constraints on the acceleration and radiation efficiencies. The observed spectral hardening in Cen A is unusual in this regard; in a "misaligned BL Lac approach" it is best understood as related to the presence of an additional emission component beyond the conventional single-zone SSC-contribution that often satisfactorily describes the SED in blazars. Apart from circumstantial evidence for the blazar Mkn 501 [46,47], Cen A is the first source where spectral results provide clear evidence for the appearance of a physically distinct component above a few GeV. Unfortunately, Cen A is a rather weak $\gamma$-ray emitting source, which significantly limits the possibilities to further probe its variability characteristics, particularly above the break. This makes it difficult to observationally disentangle the true nature of the second component with current data.

In principle a variety of different (not mutually exclusive) interpretations as to its astrophysical origin are conceivable. Related proposals in the literature operate on different scales (from a few $r_{g}$ to several $\mathrm{kpc}$ ) and include: (i) (rotational) magnetospheric models that are based on leptonic inverse Compton (IC) processes in an under-luminous accretion environment [48,49], (ii) inner (parsec-scale and below) jet scenarios that invoke differential IC scattering in a stratified jet [50], multiple SSC-emitting components moving at different angles to the line of sight [51] or photo-meson 
$(\mathrm{p} \gamma)$ interactions of UHE protons in a strong photon field [52-54] along with lepto-hadronic combinations $[55,56]$; alternatively, the hardening could be related to $\gamma$-ray induced pair-cascades in a strong disk photon field [57], a dusty torus-like region [58] or the overall host photon field [59]. Moreover, the limited angular resolution of Fermi-LAT and H.E.S.S. $(\sim 5 \mathrm{kpc})$, and the fact that no significant statistical evidence for variability has been found so far, also allows for (iii) scenarios where the emission arises on larger scales; extended scenarios in this context include the interaction of energetic protons with ambient matter (pp) in its kpc-scale region [44], the overall $\gamma$-ray contribution of a supposed population of millisecond pulsars [45], or the IC contribution by its kpc-scale jet via up-scattering off various photon fields (e.g., host galaxy starlight or CMB) [60,61], up to more extraordinary explanations invoking the self-annihilation of dark matter particles of mass $\sim 3 \mathrm{TeV}$ within a central dark matter spike [45].

While, given current knowledge, not all of these models are equally likely, and all of them come with some challenges see e.g., [62], further observational input (such as evidence for VHE variability or extension, the latter now possibly been seen [63]) is needed to better constrain them and help disclosing the real nature of this new component.

\subsection{IC 310}

The Perseus Cluster RG IC 310, located at a distance of $d \sim 80 \mathrm{Mpc}(z=0.019)$, has received particular attention in recent times. The source, originally detected by MAGIC during a campaign in 2009-2010 [64], has shown extreme VHE variability during a strong flare in November 2012, revealing VHE flux variations on timescales as short as $\Delta t \simeq 5 \mathrm{~min}$ [65], see Figure 4 . The 2012 VHE flare spectrum appears compatible with a single, hard power law of photon index $\Gamma_{\gamma} \gtrsim-2$ (and possibly as low as $\sim-1.5$ ) over a range from $70 \mathrm{GeV}$ to $8.3 \mathrm{TeV}$, with no indications of any internal absorption see also [66]. The source can reach high VHE flux levels, corresponding to an isotropic-equivalent luminosity of $L_{V H E} \simeq 2 \times 10^{44} \mathrm{erg} \mathrm{s}^{-1}$. IC 310 is commonly believed e.g., [65] to harbour a black hole of mass $M_{B H} \simeq 3 \times 10^{8} M_{\odot}$ but see also Ref. [67], for a ten times smaller estimate and has for some time been classified as a head-tail RG. The apparent lack of jet bending along with more recent indications for a one-sided pc-scale radio jet inclined at $i \lesssim 38^{\circ}$ suggests, however, that IC 310 is a transitional source at the borderline dividing low-luminosity RGs and BL Lac objects [68].

The extreme VHE variability along with the high VHE power ( $\left.\gtrsim L_{\mathrm{Edd}} / 200\right)$ and the hard $\gamma$-ray spectrum are surprising findings for a misaligned source. Based on a variety of considerations, including the orientation of its jet (probably $i \sim[10-20]^{\circ}$ ) as well as kinetic jet power and timing constraints, Aleksić et al. [65] have disfavoured several alternative models for rapid VHE variability such as magnetic reconnection e.g., [69] or jet-cloud and star interaction e.g., [70]. This inference is, however, less robust as has been shown later on cf. [71], for details. Nevertheless, the fact that the VHE flux varies on timescales $\Delta t$ much shorter than the light travel time across the black hole horizon, $r_{g}\left(3 \times 10^{8} M_{\odot}\right) / c=25 \mathrm{~min}$, has been interpreted as evidence for the occurrence of gap-type particle acceleration on sub-horizon scales, i.e., in unscreened electric field regions ("gaps") of height $h \simeq 0.2 r_{g}$ e.g., $[65,72]$. Questions concerning such an interpretation are related to the fact that the characteristic VHE power of a (steady) gap scales with the jet power, $L_{V H E} \sim L_{\mathrm{j}}\left(h / r_{g}\right)^{a}, a=2-4$ [30], the latter of which is known to be rather modest on average for IC 310, i.e., $L_{j} \sim 10^{43} \mathrm{erg} \mathrm{s}^{-1}$ cf. also, [73]. Unless strong (short-term) magnetic flux increases would occur, the expected gap output would under-predict the VHE fluxes measured during the flaring state. IC 310 has subsequently (after November 2012) shown a rather low $\mathrm{TeV}$ emission state with a steeper spectrum $(\Gamma \sim-2.4)$ measured up to $\sim 3 \mathrm{TeV}$ and with little evidence for variability. The multi-wavelength SED during this state appears to be satisfactorily reproducible with a one-zone SSC model using parameters that are comparable to those found for other misaligned, $\gamma$-ray emitting AGN [66]. 


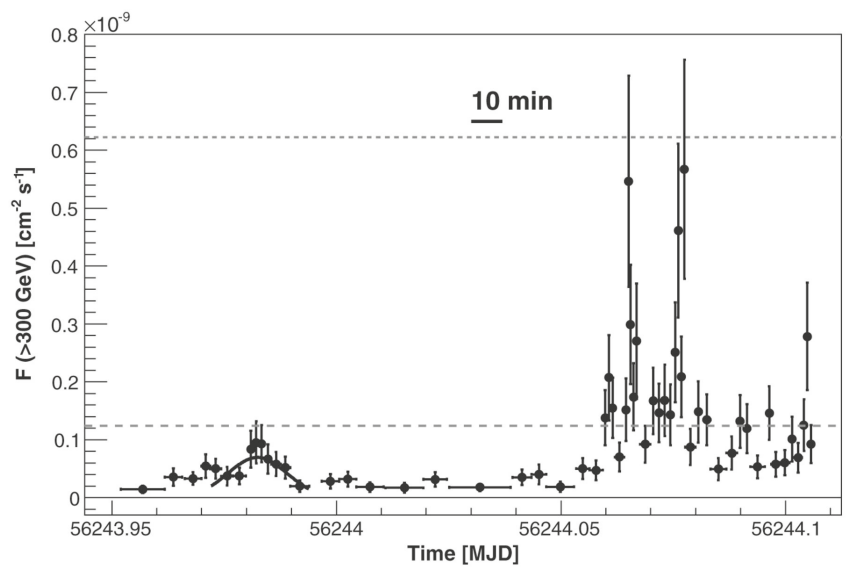

Figure 4. VHE light curve of IC 310 above $300 \mathrm{GeV}$ as observed by MAGIC during 12-13 November 2012. Rapid VHE variability on doubling timescale well below $10 \mathrm{~min}$ is apparent in the light curve. The two gray lines indicate flux levels of 1 and 5 Crab units, respectively. From Ref. [65].

\section{6. $M 87$}

The Virgo Cluster galaxy M87 (NGC 4486) has been the first extragalactic source detected at VHE energies [74]. Classified as low-excitation, weak-power FR I source, M87 hosts one of the most massive black holes of $M_{\mathrm{BH}} \simeq(2-6) \times 10^{9} M_{\odot}$ e.g., [75], and is thought to be accreting in a radiatively inefficient (RIAF) mode [76]. Given its proximity at a distance of $d \simeq 16.4 \mathrm{Mpc}$ [77] and its large mass-scale $r_{g}$, M87 has become a prominent target to probe jet formation scenarios with high-resolution radio observations down to scales of tens of gravitational radii e.g., [78-82]. Its sub-parsec scale radio jet appears misaligned by an angle $i \sim(15-25)^{\circ}$ and shows a rather complex structure, seemingly compatible with a slower, mildly relativistic $(\beta \sim 0.5 \mathrm{c})$ layer and a faster moving, relativistic spine $\left(\Gamma_{b} \sim 2.5\right)$ see e.g., [82]. Indications of a parabolic jet shape suggest that the jet initially experiences some external confinement as by a disk wind [83]. In general, the inferred jet seeds and inclinations are consistent with rather modest Doppler factors $D \lesssim$ (for review, see e.g., [84]).

At VHE energies, M87 is well known for its rapid day-scale variability (flux doubling time scales $\Delta t_{\text {obs }} \sim 1 \mathrm{~d}$ ) during active source states, and a rather hard, featureless photon spectrum compatible with a single power law (of index $\Gamma_{\gamma}=-2.2 \pm 0.2$ in high, and somewhat steeper $\Gamma_{\gamma} \sim-2.6$ in low states) extending from $\sim 300 \mathrm{GeV}$ up to $\sim 10 \mathrm{TeV}$ [85-89]. Both the observed rapid VHE variability and the hard VHE spectrum are remarkable features for a misaligned AGN, and reminiscent of those seen in IC 310. Based on the first 10 months of data, Fermi-LAT has reported HE gamma-ray emission from M87 up to $30 \mathrm{GeV}$ [90] with a photon spectrum then seemingly compatible with a single power-law of index $\Gamma_{\gamma}=-2.26 \pm 0.13$ and comparable to the one(s) in the VHE high states. Nevertheless, a simple extrapolation of this HE power-law to the VHE regime turned out to be insufficient to account for the flux levels measured during the TeV high states (up to equivalent levels of $L(>350 \mathrm{GeV}) \sim 5 \times 10^{41}$ erg/s, e.g., [88]), suggesting that the high states might be accompanied by the emergence of an additional component [84]. No evidence for significant flux variations (down to timescales of 10 days) has been found during these early HE observations, though on experimental grounds the occurrence of shorter-timescale variations cannot per se be excluded. Similar spectral results have been reported in the 3FGL catalog ( $4 \mathrm{yr}$ of data), with the HE spectrum below $10 \mathrm{GeV}$ compatible with a single power-law of $\Gamma_{\gamma}=-2.04 \pm 0.07$ [91], but with indications for a possible change above $10 \mathrm{GeV}$. The most recent analysis based on $\sim 8 \mathrm{yr}$ of Fermi-LAT data reports evidence for month-type HE variability and indications for excess emission over the standard power-law model above $\sim 10 \mathrm{GeV}$, similar to earlier findings in Cen A [92], see also Figure 5. When viewed in an HE-VHE context, these findings 
are most naturally explained by an additional emission component that dominates the highest-energy part of the spectrum and allows for a smooth HE-VHE spectral connection. As the HE spectrum extends to about $100 \mathrm{GeV}$ without indications for a cut-off and the VHE thresholds reach down to about $200 \mathrm{GeV}$, variability seen with high statistics at VHE can be used to constrain the nature of this additional component. This contrasts with Cen A where no significant VHE variability has been found yet. For M87 current findings do support proposals in which the emission arises on innermost jet scales and below.

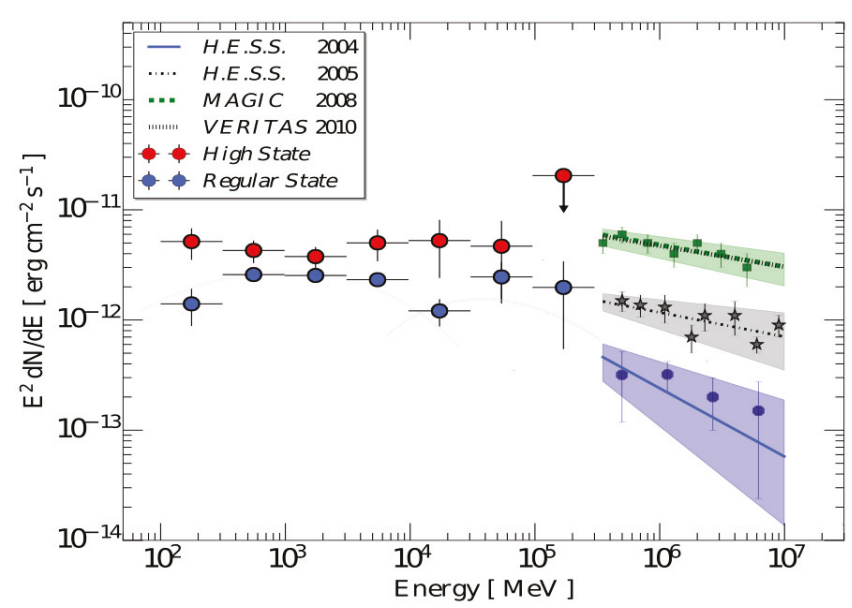

Figure 5. Gamma-ray SED for M87 based on $~ 8$ year of Fermi-LAT data including the different observed VHE states. The average ("regular") spectrum shows a break in the SED around $\sim 10 \mathrm{GeV}$, suggestive of an additional HE component. The break appears masked in the "high state" by flaring above $\sim 10 \mathrm{GeV}$. The current situation now in principle allows for a smooth connection of $\mathrm{HE}$ and VHE states. This suggests that the nature of this additional component is constrained by the observed VHE variability. The light grey curves for the two components are intended to guide the eyes only. Following Ref. [92].

Light travel time arguments in fact point to a compact VHE emission region $\left(R<c \Delta t_{\mathrm{obs}} D\right)$ in M87 of a size comparable to the Schwarzschild radius $r_{s}=(0.6-1.8) \times 10^{15} \mathrm{~cm}$ of its black hole. Similar as for Cen A, a variety of models have been introduced to account for this, cf. Figure 6 for an exemplary illustration (see Refs. [51,55,70,93-98]). The interested reader is referred to Refs. [84,99] for a more detailed description and discussion of them.

M87 has been repeatedly active over the past ten years, with VHE high states being detected in 2005, 2008 and 2010, and an elevated one (flux levels 2-3 times higher than average) in 2012. Interestingly, during all high states, day-scale VHE variability has been found. The 2012 monitoring data by VERITAS do not reveal a bright flare, but the light curve indicates VHE variability on timescales of (at least) weeks, suggesting that the often called "quiescent" state also shows some longterm evolution [100], cf. also [92]. No major VHE flare has been seen since then, though hints for day-scale variability in 2013 have been reported [101]. As the angular resolution of current VHE instruments is limited (to scales of $\sim 25 \mathrm{kpc}$ for M87), coordinated VLBI radio observations, capable of probing down to scales of tens of gravitational radii, have been performed during the 2008, 2010 and 2012 high VHE states. These results indicate that the $\mathrm{TeV}$ emission is accompanied by (delayed) radio core flux enhancements, supporting proposals that the VHE emission originate at the jet base very near to the black hole $[80,87,102,103]$. The radio-VHE correlation along with the required compactness of the VHE zone have served as a strong motivation to explore plasma injection via gap-type magnetospheric 
processes close to the black hole $[98,104,105]$. Concerning its rapid VHE variability, M87 shows some similarities with IC 310, though its associated VHE luminosity output is more than 100 times smaller than the one for IC 310.

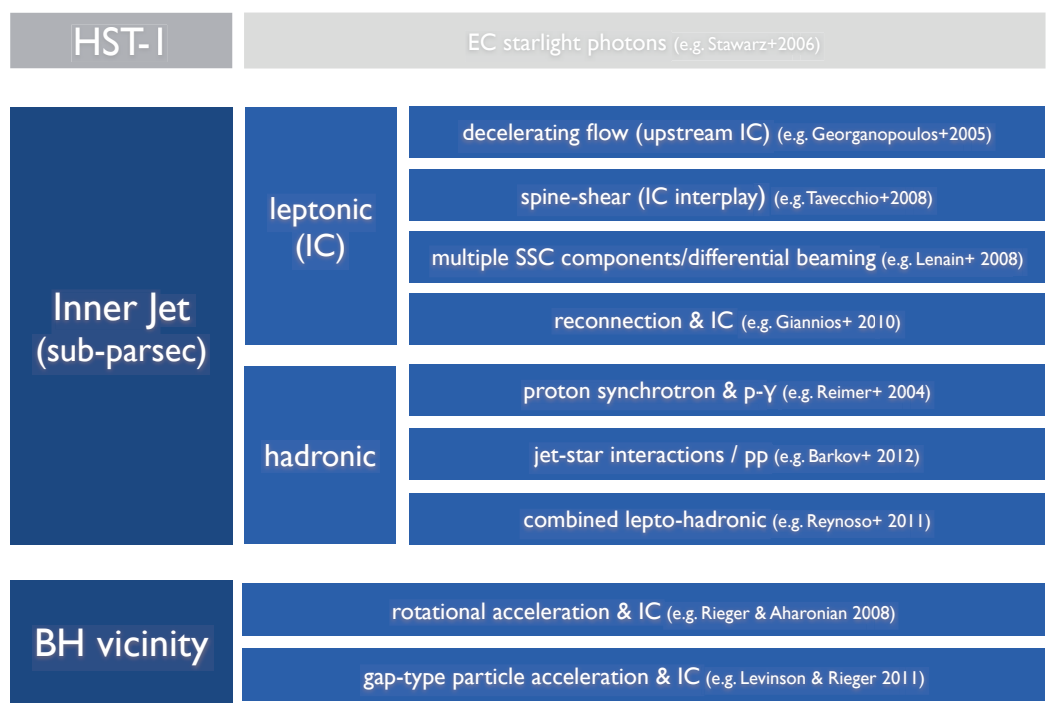

Figure 6. Possible scenarios for the origin of the variable VHE emission in M87 with exemplary references. Day-scale variability favours models on scales of the inner jet and below. "IC" refers to inverse Compton radiation.

\section{Models for the HE-VHE $\gamma$-ray Emission}

In the following we describe and comment on some recent theoretical trends and developments concerning the origin of the VHE emission beyond conventional jet (one-zone) synchrotron self-Compton (SSC) models. We distinguish between scenarios aimed at addressing the highly variable VHE part (as in M87) and those focusing on an apparently steady VHE part (as for Cen A).

\subsection{Variable VHE and Black Hole Gap Models}

The activation of Blandford-Znajek (BZ) outflows requires continuous injection of plasma in the magnetospheric region enclosed between the inner and outer light cylinders, the origin of which is yet an open issue. To fully screen out the magnetosphere, the plasma injection rate must be sufficiently high to maintain the density everywhere in the magnetosphere above the Goldreich-Julian (GJ) value. Whether the dense accretion flow surrounding the black hole can provide sufficient charges for complete screening is unclear at present; direct feeding seems unlikely, as charged particles would have to cross magnetic field lines on a timescale much shorter than the accretion time in order to reach the polar outflow. Plasma injection by virtue of macroscopic instabilities, that might lead to re-arrangement of the magnetic field configuration, is a possibility, however, the growth time of such instabilities may be much longer than the gap evacuation time, $\sim r_{g} / c$, and it is quite likely that even if occasional injection of plasma into the casual section of the magnetospheric does occur, the plasma density may not be sustained above the required level at all times. An alternative charge supply mechanism is pair creation on magnetic field lines via annihilation of $\mathrm{MeV}$ photons that emanate from a RIAF during low accretion states, or from a putative accretion disk corona during intermediate states. To estimate the $\gamma$-ray luminosity required for complete screening, we note that the density of pairs thereby created is roughly $n_{ \pm} \simeq \sigma_{\gamma \gamma} n_{\gamma}^{2} r_{g} / 3$ [98], where $\sigma_{\gamma \gamma}$ is the pair production cross section, $n_{\gamma} \simeq 10^{22} m^{-1} \tilde{R}_{\gamma}^{-2} l_{\gamma} \mathrm{cm}^{-3}$ is the 
density of MeV photons, $l_{\gamma}=L_{\gamma} / L_{E d d}$ and $R=\tilde{R} r_{g}$ are, respectively, the Eddington ratio and radius of the radiation source, and $m=M / M_{\odot}$ is the black hole mass in solar mass units. This should be compared with the GJ density, $n_{G J}=\Omega B /(2 \pi e c)=2 \times 10^{11} B_{8}\left(\Omega / \omega_{H}\right) m^{-1} \mathrm{~cm}^{-3}$, expressed here in terms of the angular velocity of magnetic surfaces $\Omega$, the angular velocity of the black hole $\omega_{H} \simeq c / 2 r_{g}$, the strength of the magnetic field near the horizon $B=10^{8} B_{8}$ Gauss, and the magnitude of the electron charge $e>0$. The requirement $n_{ \pm}>n_{G J}$ implies

$$
l_{\gamma}>10^{-3} B_{8}^{1 / 2}\left(\Omega / \omega_{H}\right)^{1 / 2}(\tilde{R} / 30)^{2} .
$$

For sources accreting in the RIAF regime, the gamma-ray luminosity can be related to the accretion rate, albeit with a large uncertainty, using an ADAF model. The strength of the magnetic field near the horizon also scales with the accretion rate, roughly as $B \simeq 10^{9}(\dot{m} / \mathrm{m})^{1 / 2} \mathrm{G}$, where $\dot{m}=\dot{M} / \dot{M}_{E d d}$, and $\dot{M}_{E d d}=10 L_{E d d} / c^{2}$. These two relations can be combined to yield the critical accretion rate below which the magnetosphere is expected to be starved,

$$
\dot{m}<4 \times 10^{-3} m^{-1 / 7} .
$$

For M87, where $m=6 \times 10^{9}$ (up to a factor of two), this implies starvation at $\dot{m} \lesssim 10^{-4}$, which seems to be above its inferred accretion rate. In sources accreting well above the critical ADAF rate, the accretion flow is anticipated to be cold, and the emission spectrum is unlikely to extend to energies above the electron mass. Gamma-rays may, nonetheless, originate from a tenuous corona, if present. No reliable constraints on the spectrum and luminosity of this coronal component have been imposed thus far. In principle, it could be that in sources that accrete at relatively high rates the magnetic field is much higher than in RIAF sources, while the gamma-ray luminosity (of the corona) is smaller. If indeed true, it could mean that gap emission in such objects may be more intense than in RIAF sources. Equation (2) has been employed to show $[30,72,98]$ that under conditions likely to prevail in many stellar and supermassive black hole systems, the annihilation rate of disk photons is insufficient to maintain the charge density in the magnetosphere at the GJ value, giving rise to formation of spark gaps [106-109]. It has been further pointed out [30,49,72,98,110-112] that the gap activity may be imprinted in the high-energy emission observed in these sources, whereby the variable $\mathrm{TeV}$ emission detected in M87 [85,87] and IC310 [65] was speculated to constitute examples of the signature of magnetospheric plasma production on horizon scales $[30,72,98,105,109,110]$. In what follows we provide a concise overview of recent black hole gap models.

\subsubsection{Stationary Gap Models}

Stationary models tacitly assume that a gap forms in a localized region of the magnetosphere outside which the ideal MHD condition (that is, $\mathbf{E} \cdot \mathbf{B}=0$ ) prevails. To gain insight it is instructive to derive the gap electrostatic equation in flat spacetime first. The generalization to Kerr spacetime then readily follows. In general, the angular velocity of magnetic field lines, $\Omega=\Omega \hat{z}$, is conserved along magnetic surfaces only in regions where the ideal MHD condition is satisfied. However, under the assumption that the gap constitutes a small disturbance in the global magnetosphere, the variation of $\Omega$ across the gap (due to the finite potential drop) can be ignored. It is then convenient to transform to a rotating coordinate system, $t^{\prime}=t, r^{\prime}=r, \theta^{\prime}=\theta$ and $\varphi^{\prime}=\varphi-\Omega t$, here in spherical coordinates, in which the electric and magnetic fields are given by $\mathbf{B}^{\prime}=\mathbf{B}, \mathbf{E}^{\prime}=\mathbf{E}+\mathbf{v} \times \mathbf{B}$ in terms of their components in the non-rotating frame (using geometric units, $c=1$ ), where $\mathbf{v}=\mathbf{\Omega} \times \mathbf{r}$ is the tangential velocity of the magnetic flux tube. It can be readily shown that in the rotating frame Gauss's law takes the form

$$
\nabla \cdot \mathbf{E}^{\prime}=4 \pi\left(\rho_{e}-\rho_{G J}\right),
$$

where $\rho_{e}$ is the charge density and $\rho_{G J}=-\nabla \cdot(\mathbf{v} \times \mathbf{B}) / 4 \pi$ denotes the Goldreich-Julian (GJ) density. In dipolar and split monopole geometries this simplifies to the well known result $\rho_{G J}=-\boldsymbol{\Omega} \cdot \mathbf{B} / 2 \pi$. 
Equation (3) indicates that a charge density $\rho_{e}=\rho_{G J}$ is needed to screen out a parallel $E_{\|}$. The generalization to Kerr geometry is straightforward. In Boyer-Lindquist coordinates, $(t, r, \theta, \varphi)$, the electric field component in the rotating coordinate frame is $F_{\mu t}^{\prime}=F_{\mu t}+\Omega F_{\mu \varphi}$, where $F_{\mu \nu}$ denotes the electromagnetic tensor, and we henceforth adopt the metric signature $(-,+,+,+)$. The GR equations can be recast in a form similar to that in flat spacetime upon using quantities measured in the local zero angular momentum observer (ZAMO) frame. For illustration, we adopt a split monopole geometry, defined by the potential $A_{\varphi}=\Psi_{H}(1-\cos \theta)$, with $\Psi_{H}$ being the magnetic flux on the horizon. The non-corotating electric field, measured by a ZAMO, is then given by $E_{r}^{\prime}=\sqrt{A} F_{r t}^{\prime} / \Sigma$ in terms of the metric functions (in geometric units) $\Sigma=r^{2}+a^{2} \cos ^{2} \theta$ and $A=\left(r^{2}+a^{2}\right)^{2}-a^{2} \Delta \sin ^{2} \theta$, with $\Delta=r^{2}+a^{2}-2 M r, M$ and $a$ being the black hole mass and specific angular momentum (spin parameter), respectively. The general relativistic Gauss's law reads:

$$
\partial_{r}\left(\sqrt{A} E_{r}^{\prime}\right)=4 \pi \Sigma\left(\rho_{e}-\rho_{G J}\right),
$$

where

$$
\rho_{G J}=\frac{\Psi_{H}}{4 \pi \sqrt{-g}} \partial_{\theta}\left[\frac{\sin ^{2} \theta}{\alpha^{2}}(\omega-\Omega)\right]
$$

is the general relativistic (GR) version of the GJ density, $\alpha=(\Sigma \Delta / A)^{1 / 2}$ is the lapse function and $\omega=2 a \mathrm{Mr} / A$ is the ZAMO angular velocity. In flat spacetime $\sqrt{A}=\Sigma=r^{2}$, and Equation (4) reduces to Equation (3) in spherical coordinates. The charge density is related to the proper densities, $n_{ \pm}$, and the time component of the four-velocities, $u_{ \pm}^{0}$, of the $\mathrm{e}^{ \pm}$pairs through $\rho_{e}=e\left(n_{+} u_{+}^{0}-n_{-} u_{-}^{0}\right)$. Equation (4) is subject to the condition $\mathbf{E}^{\prime}=0$ at the inner and outer gap boundaries, which follows from the assertion that the flow outside the gap is in a force-free state. This implies that $E_{r}^{\prime}$ does not change sign, and that $\left|E_{r}^{\prime}\right|$ has a maximum inside the gap (see inset in Figure 7). An immediate consequence is that $\rho_{G J}$ must change sign inside the gap [72,113]. Thus, the gap should form around the null surface on which $\rho_{G J}$ vanishes. Such a null surface is a distinctive feature of a Kerr black hole that results from the frame dragging effect (see Figure 7$)^{3}$.

Now, from the above it is seen that at the null surface the gap electric field scales as $\left|E_{r}^{\prime}\right| \propto h^{2}$ with the gap width $h$, and the potential as $V \propto h^{3}$. The maximum electric current flowing through the gap is limited by $j^{r}=\left|\rho_{G J}\left(r_{1}\right)\right| c \sim|d \rho / d r|_{0} h / 2$, where $r_{1}$ is the outer gap boundary and the subscript 0 designates values at the null surface. Thus, the maximum power that can be tapped, $L_{\text {gap }} \simeq 2 \pi r_{H}^{2} j V$, and, hence, the maximum gamma-ray luminosity that can be emitted by the gap, scale as $h^{4}$. A more precise expression for $L_{\text {gap }}$ is derived in Ref. [72], see also [30]. Since the gap closure condition restricts the multiplicity inside the gap to unity, the gap width $h$ increases with decreasing pair production opacity. The salient lesson is that in steady gap models the output power of the gap increases steeply with decreasing disk luminosity. Detectability of gap emission favours low luminosity sources. We shall see shortly that this is not true in case of intermittent gaps. A quantitative treatment of gap emission requires inclusion of plasma dynamics, radiation back-reaction, Compton scattering and pair production. Details are given in Refs. [72,111,113,114]. It is found that the characteristic spectrum produced in the gap consists of two components: curvature emission that peaks at sub-TeV energies, and inverse Compton emission that peaks at 10 to $100 \mathrm{TeV}$, depending on the black hole mass. Refs. $[72,111,112,114]$ predict that gap emission from stellar and supermassive black holes should be detected by upcoming experiments.

3 A null surface exists in a pulsar outer gap for other reasons. 


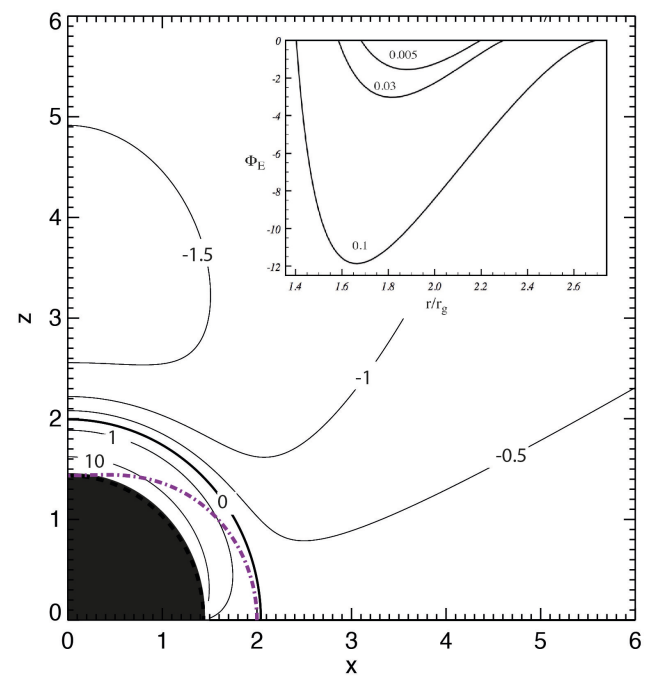

Figure 7. Contours of $\rho_{G J}$ for a split monopole geometry with $a / M=0.9, \Omega=0.5 \omega_{H}$. The numbers that label the curves are values of $\rho_{G J}(r, \theta)$, normalized by $a B_{H} / 2 \pi$, where $B_{H}=\Psi_{H} / \sqrt{A_{H}}$ is the strength of the magnetic field on the horizon. The thick solid line corresponds to the null surface on which $\rho_{G J}=0$. The black circle delineates the interior of the black hole, and the purple dashed-dotted line marks the static surface. The inset shows solutions for the electric flux, $\Phi_{E}=\sqrt{A} E_{r}^{\prime}$, computed from Equation (4) with $\rho_{e}<<\rho_{G J}$ along a magnetic surface inclined at $\theta=30^{\circ}$, in a magnetosphere of a supermassive black hole of mass $M=10^{9} \mathrm{M}_{\odot}$, for $B_{H}=10^{4} \mathrm{G}$.

\subsubsection{Time-Dependent Models}

The main difficulty with steady gap solutions for the null surface is that they exist only under highly restricted conditions, that may not apply to most objects [113]. The reason is that the gap closure condition (i.e., unit multiplicity) imposes a relation between the global magnetospheric current (through the gap width) and the pair production opacity, which in reality are two independent and unrelated quantities. An exemplary illustration for the existence regime of steady gap solutions is displayed in Figure 8, for a supermassive black hole of mass $m=10^{9}$ and a power-law disk emission spectrum. Similar results are obtained for stellar mass black holes. This example indicates that in practice, steady gaps can form only in sources with extremely low Eddington ratios. Additional difficulty stems from an inconsistency between the flow direction at the outer gap boundary and the ideal MHD flow below the stagnation surface $[113,115]$. Finally, the stability of steady gaps is questionable. The main conclusion is that sparking of starved magnetospheric regions is likely to be inherently intermittent.

Attempts to construct 1D time-dependent models have been reported recently [116,117]. The analysis described in Ref. [116] was performed using a newly developed, fully GR (in Kerr geometry) particle-in-cell code that implements Monte-Carlo methods to compute the interaction of pairs and gamma-rays with the soft photons emitted by the accretion flow. As in the steady models mentioned above, the gap is assumed to be a small disturbance that does not affect the global magnetospheric structure. The evolution of the electric field is governed by the equation

$$
\partial_{t}\left(\sqrt{A} E_{r}^{\prime}\right)=-4 \pi\left(\Sigma j^{r}-J_{0}\right),
$$

that generalizes the flat spacetime model derived in Ref. [118]. Here $j^{r}=e\left(n_{+} u_{+}^{r}-n_{-} u_{-}^{r}\right)$ is the radial electric current density, expressed in terms of the proper electron $\left(n_{-}\right)$and positron $\left(n_{+}\right)$ densities and their four-velocities $u_{-}$and $u_{+}$, and $J_{0}$ represents the global magnetospheric current, 
which is an input parameter of the model in addition to $\Omega$. The initial condition, $E_{r}^{\prime}(t=0, r)$, is obtained upon solving Equation (4) at the beginning of each run. For details the reader is referred to Ref. [116]. The analysis indicates that when the Thomson length for collision with disk photons becomes smaller than the gap width, i.e., $\tau_{0}>1$, screening of the gap occurs, following a prompt discharge phase that exhausts the initial energy stored in the gap, through low amplitude, rapid plasma oscillations that produce self-sustained pair cascades, with quasi-stationary pair and gamma-ray spectra. An example is exhibited in Figures 9 and 10. The gamma-ray spectrum emitted from the gap peaks in the $\mathrm{TeV}$ band (Figure 10), with a total luminosity that constitutes a fraction of about $10^{-5}$ of the corresponding Blandford-Znajek power. This seems different than the spectrum predicted by steady gap models. As those simulations are demanding, only a small range of parameters was explored in [116]. Future studies should investigate how the emission properties depend on the black hole mass, the target radiation spectrum and the magnetic field strength.

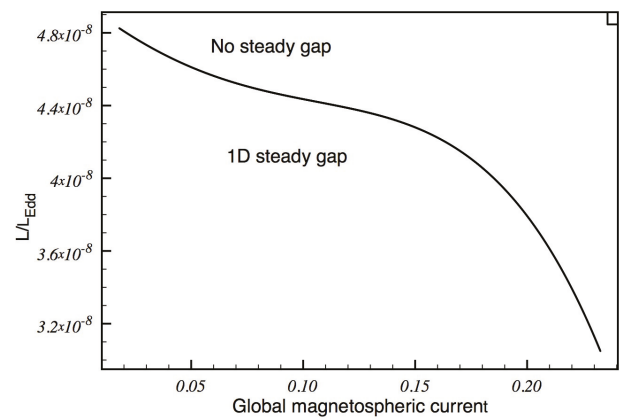

Figure 8. Maximum Eddington ratio below which local steady gap solutions exist, versus normalized magnetospheric current, $2 \pi J_{0} /\left(\Sigma_{\text {null }} \Omega B_{H} \cos \theta\right)$, where $\Sigma_{\text {null }}$ is the value of $\Sigma$ on the null surface, for a supermassive black hole of mass $M_{B H}=10^{9} M_{\odot}$, magnetic field $B=10^{4} \mathrm{G}$, a radiation source of size $R=30 r_{g}$ and a power law spectrum, $I_{v} \propto v^{-2}$, with a low energy cutoff at $v_{\text {min }}=10^{12} \mathrm{~Hz}$, and inclination angle $\theta=30^{\circ}$. (See Ref. [113] for further details).
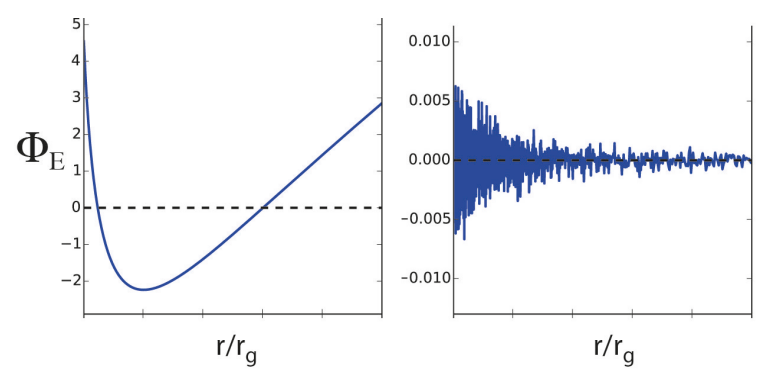

Figure 9. Evolution of the gap electric field in a magnetosphere of a supermassive black hole with the same parameters as in Figure 8. The left panel shows the electric flux $\Phi_{E}=\sqrt{A} E_{r}^{\prime}$ at the initial time $t=0$. The right panel shows the electric flux at time $t=20 \mathrm{rg}_{\mathrm{g}} \mathrm{c}$, roughly the onset of the quasi-steady oscillations. Note the change of scale on the vertical axis between the two panels. The amplitude of $E_{r}^{\prime}$ during the quasi-steady oscillations is about $10^{-3}$ of its initial magnitude at the null surface. The fiducial opacity in this case is $\tau_{0}=10$.

A caveat of the model described above is that it does not account for the effect of the ideal MHD fields on the dynamics of the plasma. An attempt to include such additional forces has been made in Ref. [117], where it has been argued that the gap dynamics is cyclic in nature rather than quasi-steady. However, the analysis in this work is based on a mirror gap model in flat spacetime, that inherently 
assumes the presence of a current sheet (or current side flow) at the stagnation surface (by reversal of the toroidal magnetic field there). Whether this model is a reasonable representation of a starved Kerr black hole magnetosphere remains to be demonstrated. A cyclic behaviour is naively anticipated in a global model that links the gap and the magnetosphere together [113]. How this would affect the spectrum and light curves of the HE gap emission is yet an open issue that can only be studied using 2D simulations.
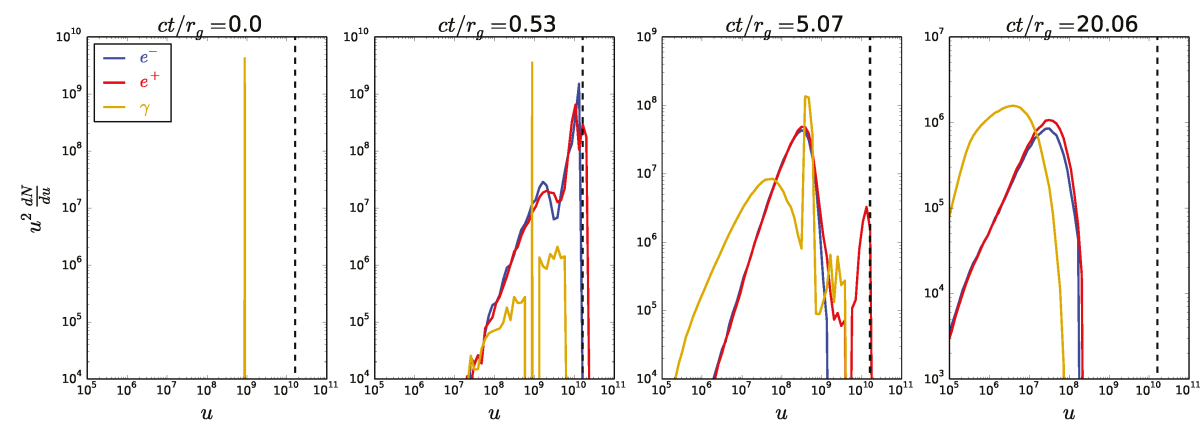

Figure 10. Evolution of the electron (blue), positron (red) and photon (yellow) spectra for the case shown in Figure 9. The photon energy is given in $m_{e} c^{2}$ units. The peak of the quasi-steady gamma-ray spectrum in this example (rightmost panel) is at $\sim 1 \mathrm{TeV}$.

\subsection{Variable VHE and Inner Jet Models}

Rapid variability on timescales of days and below implies a compact emitting region, and is often taken to indicate that this emitting region is located at sub-parsec jet scales $\left(<10^{4} r_{g}\right)$ and below. Promising jet-related proposals in this context (beyond black hole gaps) include the following (cf. Figure 11).
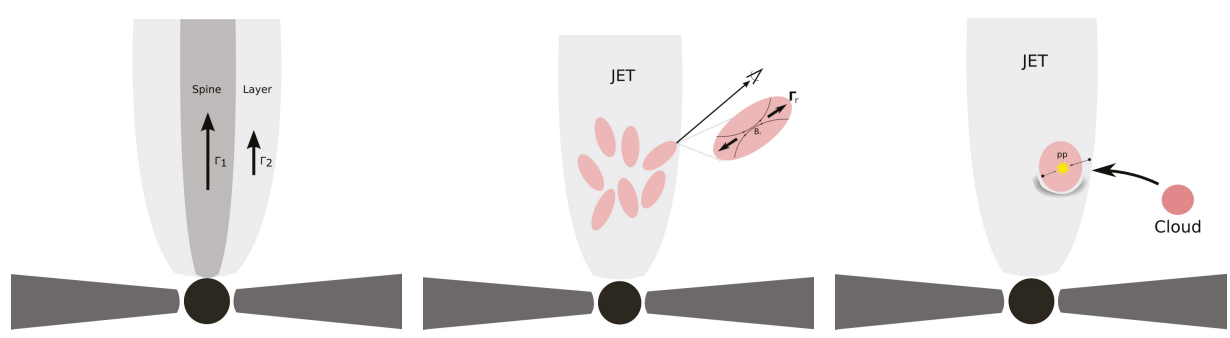

Figure 11. Possible scenarios for the origin of rapidly variable VHE emission on scales of the inner jet, i.e., beyond black hole magnetospheric emission. Left: Illustration of a stratified jet composed of a fast spine - slow layer [93]. Radiative interplay (external IC) can increase the $\gamma$-ray luminosity of each component. Middle: Illustration of a jets-in-jet model [94], where a variety of 'mini-jet' features (plasmoids) are triggered by magnetic reconnection events within the main jet flow. This could lead to an additional velocity component relative to the main flow $\left(\Gamma_{r}\right)$ and allow a favorable orientation with respect to the observer. Right: Illustration of a hadronic model, where interactions of the jet with a massive obstacle (star or cloud) facilitates shock-acceleration and introduces a sufficient target density to allow for efficient $p p$-collisions [70].

\subsubsection{Spine-Shear Scenarios}

The complex interplay of black hole- and disk-driven outflows along with environmental interaction (e.g., entrainment) is likely to give rise to a non-uniform flow topology where different 
jet layers possess different bulk flow speeds. In its simplest realization a fast $\left(\Gamma_{1} \gg 1\right)$ spine is taken to be surrounded by a slower-moving $\left(\Gamma_{2}<\Gamma_{1}\right)$ sheath or layer e.g., [50]. This is essentially a two-zone model, yet with parameter-space constrained by the requirement of an efficient radiative interplay. Depending on the viewing angle, we may see different parts of the jet (spine or sheath), resulting in different emission characteristics for misaligned AGN when compared with those of blazars. In particular, given their larger inclination angles, the VHE emission in RGs would usually be dominated by the stronger Doppler-boosted emission from the layer. VHE variability then essentially imposes constraints on the size of the layer. Spine-sheath models can in principle accommodate fast variability and facilitate an increased gamma-ray luminosity as each component sees the (external) radiation of the other amplified by the relative motion between them $\left(\Gamma_{\text {rel }}=\Gamma_{1} \Gamma_{2}\left[1-\beta_{1} \beta_{2}\right]\right)$, thereby enhancing the IC contribution of both its spine and its layer $[31,50,93]$. In order for this interplay to work efficiently however, the emitting zones need to be (quasi) co-spatial. This in turn leads to transparency issues as long as one aims at a simultaneous reproduction of the infrared-optical part of the SED, since the large gamma-ray opacity associated with the intense (weakly Doppler-boosted) radiation field of the spine usually results in considerable $\gamma \gamma$-absorption and a steep slope at $\mathrm{TeV}$ energies. Hard $\mathrm{TeV}$ spectra thus cannot simply be accounted for with such models e.g., [24]. Nevertheless, an internal velocity stratification (shear) bears the potential to solve several issues regarding the unification of BL Lacs and RGs [119-121]. The noted emission models represent a simplified first approach, that focus on the radiative interplay only and do not yet take any acceleration effects e.g., [122] nor time-dependencies into account. Decoupling of the infrared and VHE emission parts would alleviate some tensions, though it would leave a larger part of the parameter space unconstrained. Similar to other two-zone approaches, the non-thermal emission can in many cases be reproduced under equipartition conditions which may be counted in its favour [123]. Time-dependent extensions are certainly needed to further assess its potential in the context of RG modelling.

\subsubsection{Reconnection—Mini-Jets and Plasmoids}

Relativistic jets are generally expected to be initially magnetically dominated (magnetization $\sigma_{m} \gg 1$ ), and this has motivated studies where the field energy is released via collisionless magnetic reconnection allowing for non-thermal particle acceleration and subsequent high energy gamma-ray (IC) production e.g., [69,94,124-129]. An open question at present concerns whether formation of current sheets on sufficiently small scales can result from current driven instabilities induced during the propagation of the jet e.g., [130-132], or may inherently form during injection of the jet, e.g., due to advection of asymmetric magnetic field or magnetic loops by the disk e.g., [133,134]. The observed collimation profile of M87 seems to indicate that in this source the jet is kink stable [83]. Upcoming Event Horizon Telescope (EHT) observations on horizon scale may shed more light on this issue.

Reconnection-related models of the type proposed in [94] assume that relativistic (Petschek-type) reconnection occurs in the jets of RGs (taking them to be electron-proton dominated with $\sigma_{m} \sim 100$ ), leading to efficient electron acceleration and the generation of variable VHE gamma-rays via inverse Compton (SSC and/or EC) scattering e.g., [135]. Moreover, efficient reconnection allows for an additional relativistic velocity component of the ejected plasma $\left(\Gamma_{r} \simeq \sqrt{\sigma_{m}}\right)$ relative to the mean bulk flow of the jet, and directed at some angle to it. Given multiple, localised reconnection sites ("mini-jets") within the jet, strong differential Doppler boosting effects $(D \gg 1)$ could become possible even for RG sources whose main jet direction are substantially misaligned. This could then provide a simple explanation for ultra-fast variability in misaligned AGN. Caveats concern whether such high magnetisations should indeed be expected for electron-proton (disk-driven) jets, and whether the impact of a magnetic guide field, that would lead to weak dissipation only [136], can be neglected on the anticipated scale of VHE gamma-ray production. Nevertheless, reconnection is particularly interesting as it could facilitate non-thermal particle energization in those magnetized part of the jets where diffusive shock acceleration is inefficient e.g., [137], and ensure a rough equipartition between magnetic fields and radiating particles in the reconnection downstream (emitting) region [128]. In the 
absence of a guide field, relativistic reconnection results in a flat (hard) power-law tail $n(\gamma) \sim \gamma^{-\alpha}$ with slope $\alpha<2$ for $\sigma_{m}>10$, most probably approaching $\alpha \sim 1$ in the extreme relativistic case [129,138,139]. For large Lundquist numbers the reconnection layer becomes unstable to resistive (tearing) instabilities, causing its fragmentation into local magnetic islands or plasmoids. More recent studies have thus concentrated on characterizing the (fractal-like nature of) plasmoid formation in relativistic pair plasmas $[140,141]$.

\subsubsection{Jet-Star and Cloud Interactions}

The ubiquity of stars and gas in the central region of the AGN host galaxy can lead to frequent jet-star/cloud interactions, facilitate jet entrainment and mass loading by stellar winds e.g., [142], and give rise to knotty X-ray structures as e.g., seen in the large-scale jet of Cen A [143]. Over the last few years a variety of studies have explored its impact on the gamma-ray emission characteristics in AGN, both for its steady and transient/flaring states e.g., [144-152]. In the RG context, one interesting application concerns the possible contribution of inelastic proton-proton (pp) collisions in the generation of variable VHE emission. While AGN jets are commonly considered not to carry enough target matter $\left(n_{p}\right)$ to allow for efficient pp-collisions (given its long timescale $t_{p p} \simeq 10^{15} / n_{p} \mathrm{~s}$ ), interactions of a red giant star or a massive, dense gas cloud (size $r_{o}$ ) with the base of the jet (radius $r_{j}$ ) could occasionally introduce a high amount of matter, trigger shock acceleration and potentially drive rapid VHE activity [70,144]. Model calculations in the case of M87 [153] suggest that such a scenario could account for the observed VHE characteristics (including day-scale variability) if the jet would be powerful enough and a sufficiently large fraction $\left(\propto r_{o}^{2} / r_{j}^{2}\right)$ of it could be channeled into VHE $\gamma$-ray production. Given the observed large opening angle and transversal dimension $r$ of the milli-arcsec radio jet in M87, the latter is not obvious. In fact, simple models of this type often need jet powers in excess of current estimates. In principle, however, these power constraints could be somewhat relaxed if the jets possess a spine-shear-type configuration with most of the energy flux concentrated into a narrow core. This would then bear some similarities with the set-up discussed in Section 3.2.1. In addition, the effective size $r_{0}$ (if related to shocks at quite some distance from the star) could well be larger than the initial size of the obstacle e.g., [148,149]. While the availability of suitable stellar orbits on sub-parsec scales limits the possible recurrence (frequency) of short, star-driven VHE flaring events, multiple collisions along the jet seem unavoidable. Depending on conditions in the host galaxy, this could also result in a detectable, steady $\gamma$-ray contribution at VHE energies; a recent calculation of the cumulative emission from multiple jet-stellar wind interactions in M87, however, suggests this to be too low to account for its overall VHE flux levels [152].

\subsection{Steady VHE and Extended Jet Models}

The detection of extended X-ray emission from the large-scale jets in AGN by Chandra ${ }^{4}$ has raised the possibility that these jets are also steady sources of VHE $\gamma$-rays. Electron synchrotron radiation is by now the favoured interpretation for the X-ray emission e.g., [154,155], indicating the presence of highly energetic electrons with Lorentz factors up to $\gamma \sim 10^{8}(100 \mu \mathrm{G} / B)^{1 / 2} D^{-1 / 2}$, where $D$ is the Doppler factor and $B$ the large-scale magnetic field strength. While diffusive shock acceleration in the jet could in principle facilitate such energies, localized (shock-type) acceleration at knots is usually not sufficient given the fact that there is little evidence for e.g. the inter-knot regions to have significantly steeper spectra than the adjacent knots as one would expect in the case of synchrotron cooling. This may point to the operation of a continued or distributed acceleration mechanisms such a stochastic or shear particle acceleration e.g., [156].

Figure 12 shows a SED result of a recent spectral analysis in M87 [157]. The difference of the radio and X-ray spectral indices supports a synchrotron as opposed to an IC origin.

4 https://hea-www.harvard.edu/XJET/. 
The multi-TeV electrons in the jets will then also up-scatter the host starlight, dust, EBL or CMB photons to $\gamma$-ray energies, resulting in a weak and steady VHE contribution that may become detectable in nearby RG. IC up-scattering of CMB $\left(u_{C M B}=4.2 \times 10^{-13} \mathrm{erg} \mathrm{cm}^{-3} ; v_{p} \simeq 1.6 \times 10^{11} \mathrm{~Hz}\right)$, for example, leads to a non-reducible multi-TeV contribution at flux levels of $f_{I C} \sim\left(u_{I C} / u_{B}\right) f_{x} \sim 10^{-3} f_{x}$. For M87 however, the total X-ray flux of the knots is of the order $f_{x} \sim 10^{-12} \mathrm{erg} \mathrm{cm}^{-2} \mathrm{~s}^{-1}$ only, implying an IC-CMB contribution at flux levels well below current detections. Up-scattering of dust or starlight photons on the other hand, is expected to yield a much higher VHE contribution that could be probed with CTA [61]. Given the (current) absence of VHE variability in Cen A, an extended (leptonic) origin of its VHE emission certainly cannot be excluded. In addition, the cumulative IC emission from multiple jet-star collisions in the kpc-scale jet [148] might further contribute. Evidence for a possible VHE extension has in fact been recently reported for Cen A [63]. We note that an experimental verification of extended VHE emission would support the notion that the large-scale jets in AGN could make a relevant contribution to the $\mathrm{TeV}$ background when compared to the highly-boosted VHE emission from blazar cores [155].

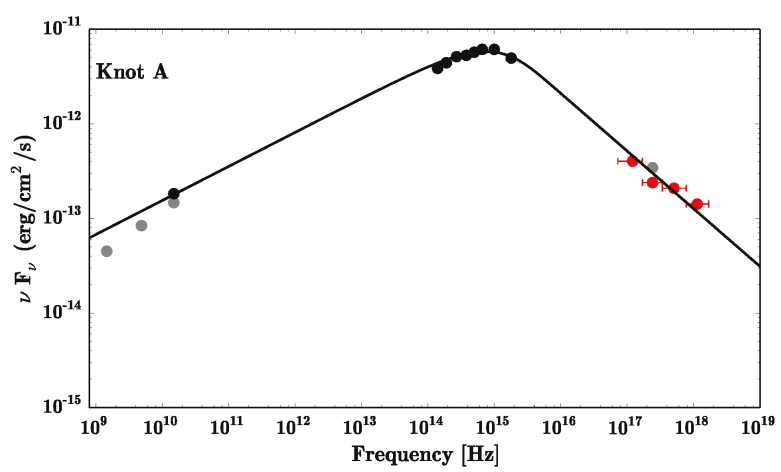

Figure 12. Representative multi-wavelength SED for the outer knot A in the kpc-scale jet of M87 including X-ray emission (red) based on $1.5 \mathrm{Msec}$ of Chandra data. The emission is thought to be synchrotron in origin, indicating the presence of electrons up to multi-TeV energies within the jet. Inverse Compton up-scattering off various soft photon fields (starlight, dust, EBL, CMB) would result in a steady VHE contribution. From Ref. [157].

\section{Conclusions}

The experimental progress over the last decade has led to the discovery of radio galaxies (RGs) at $\gamma$-ray energies, revealing exceptional features such as spectral hardening or ultra-fast variability. With their jets misaligned and related Doppler boosting effects only modest, RGs are offering unique insights into physical mechanisms and environments (e.g., the plasma physics of jets or the black hole vicinity) that are otherwise difficult to access.

The unexpected spectral hardening at gamma-ray energies seen in Cen A, for example, points to the emergence of a new physical component beyond the conventional SSC-type one, with current interpretations ranging from the smallest (sub-pc) to the largest (kpc) jet scales, up to extended dark matter scenarios. On the other hand, rapid VHE variability on timescales shorter or comparable to the light travel time across the horizon of the black hole, as e.g., seen in IC 310 and M87, provides evidence for a highly compact emitting zone, probably at the very origin of the jet itself (black hole gaps), or internally (reconnection) or externally (star collision) induced.

These and related experimental findings at gamma-ray energies have been vital in triggering important conceptual progress in black hole-jet physics and generated a variety of promising research avenues. Along with further theoretical efforts, dedicated observational studies are currently needed to, 
for example, clarify source classification and to better resolve their timing characteristics. The upcoming CTA array [158] will have the potential to probe deeper into the spectral and variability characteristics of RGs, and thereby allow to fundamentally advance our understanding of the AGN phenomena in general.

Funding: FMR acknowledges funding by a DFG Heisenberg Fellowship RI 1187/6-1. AL acknowledges support by the Israel Science Foundation (grant 1114/17).

Acknowledgments: FMR acknowledges the support and hospitality of the MPIK Heidelberg.

Conflicts of Interest: The authors declare no conflict of interest.

\section{References}

1. Barthel, P.D. Is every quasar beamed? Astrophys. J. 1989, 336, 606-611. [CrossRef]

2. Antonucci, R. Unified models for active galactic nuclei and quasars. Annu. Rev. Astron. Astrophys. 1993, 31, 473-521. [CrossRef]

3. Fanaroff, B.L.; Riley, J.M. The morphology of extragalactic radio sources of high and low luminosity. Mon. Not. R. Astron. Soc. 1974, 167,31P-36P. [CrossRef]

4. Ghisellini, G.; Celotti, A. The dividing line between FR I and FR II radio-galaxies. Astron. Astrophys. 2001, 379, L1-L4. [CrossRef]

5. Wang, J.M.; Ho, L.C.; Staubert, R. The central engines of radio-loud quasars. Astron. Astrophys. 2003, 409, 887-898. [CrossRef]

6. Urry, C.M.; Padovani, P. Unified Schemes for Radio-Loud Active Galactic Nuclei. Publ. Astron. Soc. Pac. 1995, 107, 803. [CrossRef]

7. Kollgaard, R.I.; Wardle, J.F.C.; Roberts, D.H.; Gabuzda, D.C. Radio constraints on the nature of BL Lacertae objects and their parent population. Astron. J. 1992, 104, 1687-1705. [CrossRef]

8. Antonucci, R. A panchromatic review of thermal and nonthermal active galactic nuclei. Astron. Astrophys. Trans. 2012, 27, 557-602.

9. Rector, T.A.; Stocke, J.T.; Perlman, E.S.; Morris, S.L.; Gioia, I.M. The Properties of the X-Ray-selected EMSS Sample of BL Lacertae Objects. Astron. J. 2000, 120, 1626-1647. [CrossRef]

10. Sahakyan, N.; Baghmanyan, V.; Zargaryan, D. Fermi-LAT observation of nonblazar AGNs. Astron. Astrophys. 2018, 614, A6. [CrossRef]

11. Chiaberge, M.; Capetti, A.; Celotti, A. The BL Lac heart of Centaurus A. Mon. Not. R. Astron. Soc. 2001, 324, L33-L37. [CrossRef]

12. HESS Collaboration; Abdalla, H.; Abramowski, A.; Aharonian, F.; Ait Benkhali, F.; Akhperjanian, A.G.; Andersson, T.; Angüner, E.O.; Arrieta, M.; Aubert, P.; et al. H.E.S.S. discovery of very high energy $\gamma$-ray emission from PKS 0625-354. Mon. Not. R. Astron. Soc. 2018, 476, 4187-4198. [CrossRef]

13. Mingo, B.; Hardcastle, M.J.; Croston, J.H.; Dicken, D.; Evans, D.A.; Morganti, R.; Tadhunter, C. An X-ray survey of the $2 \mathrm{Jy}$ sample - I. Is there an accretion mode dichotomy in radio-loud AGN? Mon. Not. R. Astron. Soc. 2014, 440, 269-297. [CrossRef]

14. Wills, K.A.; Morganti, R.; Tadhunter, C.N.; Robinson, T.G.; Villar-Martin, M. Emission lines and optical continuum in low-luminosity radio galaxies. Mon. Not. R. Astron. Soc. 2004, 347, 771-786. [CrossRef]

15. Ramos Almeida, C.; Tadhunter, C.N.; Inskip, K.J.; Morganti, R.; Holt, J.; Dicken, D. The optical morphologies of the 2 Jy sample of radio galaxies: Evidence for galaxy interactions. Mon. Not. R. Astron. Soc. 2011, 410, 1550-1576. [CrossRef]

16. Müller, C.; Krauss, F.; Kadler, M.; Trüstedt, J.; Ojha, R.; Ros, E.; Wilms, J.; Böck, M.; Dutka, M.; Carpenter, B.; et al. The TANAMI Program: Southern-Hemisphere AGN on (Sub-)parsec Scales. arXiv 2013, arXiv:1301.4384.

17. Fukazawa, Y.; Finke, J.; Stawarz, Ł.; Tanaka, Y.; Itoh, R.; Tokuda, S. Suzaku Observations of $\gamma$-Ray Bright Radio Galaxies: Origin of the X-Ray Emission and Broadband Modeling. Astrophys. J. 2015, 798, 74. [CrossRef]

18. Mukherjee, R. VERITAS discovery of VHE emission from the FRI radio galaxy 3C 264. The Astronomer's Telegram, March 2018. Available online: http:/ /adsabs.harvard.edu/abs/2018ATel11436....1M (accessed on 5 October 2018). 
19. De Ruiter, H.R.; Parma, P.; Fanti, R.; Fanti, C. Far-UV to mid-IR properties of nearby radio galaxies. Astron. Astrophys. 2015, 581, A33. [CrossRef]

20. Ajello, M.; Atwood, W.B.; Baldini, L.; Ballet, J.; Barbiellini, G.; Bastieri, D.; Bellazzini, R.; Bissaldi, E.; Blandford, R.D.; Bloom, E.D.; et al. 3FHL: The Third Catalog of Hard Fermi-LAT Sources. Astrophys. J. Suppl. Ser. 2017, 232, 18. [CrossRef]

21. Meyer, E.T.; Georganopoulos, M.; Sparks, W.B.; Perlman, E.; van der Marel, R.P.; Anderson, J.; Sohn, S.T.; Biretta, J.; Norman, C.; Chiaberge, M. A kiloparsec-scale internal shock collision in the jet of a nearby radio galaxy. Nature 2015, 521, 495-497. [CrossRef] [PubMed]

22. Aleksić, J.; Alvarez, E.A.; Antonelli, L.A.; Antoranz, P.; Asensio, M.; Backes, M.; Barres de Almeida, U.; Barrio, J.A.; Bastieri, D.; Becerra González, J.; et al. Detection of very-high energy $\gamma$-ray emission from NGC 1275 by the MAGIC telescopes. Astron. Astrophys. 2012, 539, L2. [CrossRef]

23. Aleksić, J.; Ansoldi, S.; Antonelli, L.A.; Antoranz, P.; Babic, A.; Bangale, P.; Barres de Almeida, U.; Barrio, J.A.; Becerra González, J.; Bednarek, W.; et al. Contemporaneous observations of the radio galaxy NGC 1275 from radio to very high energy $\gamma$-rays. Astron. Astrophys. 2014, 564, A5. [CrossRef]

24. MAGIC Collaboration; Ansoldi, S.; Antonelli, L.A.; Arcaro, C.; Baack, D.; Babić, A.; Banerjee, B.; Bangale, P.; Barres de Almeida, U.; Barrio, J.A.; et al. Gamma-ray flaring activity of NGC1275 in 2016-2017 measured by MAGIC. Astron. Astrophys. 2018, 617, A91. [CrossRef]

25. Wilman, R.J.; Edge, A.C.; Johnstone, R.M. The nature of the molecular gas system in the core of NGC 1275. Mon. Not. R. Astron. Soc. 2005, 359, 755-764. [CrossRef]

26. Wu, Q.; Cao, X.; Wang, D.X. Evidence for Rapidly Rotating Black Holes in Fanaroff-Riley I Radio Galaxies. Astrophys. J. 2011, 735, 50. [CrossRef]

27. Walker, R.C.; Romney, J.D.; Benson, J.M. Detection of a VLBI counterjet in NGC 1275: A possible probe of the parsec-scale accretion region. Astrophys. J. 1994, 430, L45-L48. [CrossRef]

28. Fujita, Y.; Nagai, H. Discovery of a new subparsec counterjet in NGC 1275: the inclination angle and the environment. Mon. Not. R. Astron. Soc. 2017, 465, L94-L98. [CrossRef]

29. Fujita, Y.; Kawakatu, N.; Shlosman, I.; Ito, H. The young radio lobe of 3C 84: Inferred gas properties in the central 10 pc. Mon. Not. R. Astron. Soc. 2016, 455, 2289-2294. [CrossRef]

30. Katsoulakos, G.; Rieger, F.M. Magnetospheric Gamma-Ray Emission in Active Galactic Nuclei. Astrophys. J. 2018, 852, 112. [CrossRef]

31. Tavecchio, F.; Ghisellini, G. On the spine-layer scenario for the very high-energy emission of NGC 1275. Mon. Not. R. Astron. Soc. 2014, 443, 1224-1230. [CrossRef]

32. Neumayer, N. The Supermassive Black Hole at the Heart of Centaurus A: Revealed by the Kinematics of Gas and Stars. Publ. Astron. Soc. Aust. 2010, 27, 449-456. [CrossRef]

33. Whysong, D.; Antonucci, R. Thermal Emission as a Test for Hidden Nuclei in Nearby Radio Galaxies. Astrophys. J. 2004, 602, 116-122. [CrossRef]

34. Meisenheimer, K.; Tristram, K.R.W.; Jaffe, W.; Israel, F.; Neumayer, N.; Raban, D.; Röttgering, H.; Cotton, W.D.; Graser, U.; Henning, T.; et al. Resolving the innermost parsec of Centaurus A at mid-infrared wavelengths. Astron. Astrophys. 2007, 471, 453-465. [CrossRef]

35. Fürst, F.; Müller, C.; Madsen, K.K.; Lanz, L.; Rivers, E.; Brightman, M.; Arevalo, P.; Baloković, M.; Beuchert, T.; Boggs, S.E.; et al. NuSTAR and XMM-Newton Observations of the Hard X-Ray Spectrum of Centaurus A. Astrophys. J. 2016, 819, 150. [CrossRef]

36. Müller, C.; Kadler, M.; Ojha, R.; Perucho, M.; Großberger, C.; Ros, E.; Wilms, J.; Blanchard, J.; Böck, M.; Carpenter, B.; et al. TANAMI monitoring of Centaurus A: The complex dynamics in the inner parsec of an extragalactic jet. Astron. Astrophys. 2014, 569, A115. [CrossRef]

37. Aharonian, F.; Akhperjanian, A.G.; Anton, G.; de Almeida, U.B.; Bazer-Bachi, A.R.; Becherini, Y.; Behera, B.; Benbow, W.; Bernlöhr, K.; Boisson, C.; et al. Discovery of Very High Energy $\gamma$-Ray Emission from Centaurus a with H.E.S.S. Astrophys. J. 2009, 695, L40-L44. [CrossRef]

38. HESS Collaboration; Abdalla, H.; Abramowski, A.; Aharonian, F.; Ait Benkhali, F.; Angüner, E.O.; Arakawa, M.; Armand, C.; Arrieta, M.; Backes, M.; et al. The $\gamma$-ray spectrum of the core of Centaurus A as observed with H.E.S.S. and Fermi-LAT. arXiv 2018, arXiv:1807.07375.

39. Abdo, A.A.; Ackermann, M.; Ajello, M.; Atwood, W.B.; Baldini, L.; Ballet, J.; Barbiellini, G.; Bastieri, D.; Baughman, B.M.; Bechtol, K.; et al. Fermi Large Area Telescope View of the Core of the Radio Galaxy Centaurus A. Astrophys. J. 2010, 719, 1433-1444. [CrossRef] 
40. Abdo, A.A.; Ackermann, M.; Ajello, M.; Atwood, W.B.; Baldini, L.; Ballet, J.; Barbiellini, G.; Bastieri, D.; Baughman, B.M.; Bechtol, K.; et al. Fermi Gamma-Ray Imaging of a Radio Galaxy. Science 2010, 328, 725. [CrossRef] [PubMed]

41. Yang, R.Z.; Sahakyan, N.; de Ona Wilhelmi, E.; Aharonian, F.; Rieger, F. Deep observation of the giant radio lobes of Centaurus A with the Fermi Large Area Telescope. Astron. Astrophys. 2012, 542, A19. [CrossRef]

42. Sun, X.N.; Yang, R.Z.; Mckinley, B.; Aharonian, F. Giant lobes of Centaurus A as seen in radio and $\gamma$-ray images obtained with the Fermi-LAT and Planck satellites. Astron. Astrophys. 2016, 595, A29. [CrossRef]

43. Ackermann, M.; Ajello, M.; Baldini, L.; Ballet, J.; Barbiellini, G.; Bastieri, D.; Bellazzini, R.; Bissaldi, E.; Blandford, R.D.; Bloom, E.D.; et al. Fermi Large Area Telescope Detection of Extended Gamma-Ray Emission from the Radio Galaxy Fornax A. Astrophys. J. 2016, 826, 1. [CrossRef]

44. Sahakyan, N.; Yang, R.; Aharonian, F.A.; Rieger, F.M. Evidence for a Second Component in the High-energy Core Emission from Centaurus A? Astrophys. J. 2013, 770, L6. [CrossRef]

45. Brown, A.M.; Boehm, C.; Graham, J.; Lacroix, T.; Chadwick, P.; Silk, J. Discovery of a new extragalactic population of energetic particles. Phys. Rev. D 2017, 95, 063018. [CrossRef]

46. Neronov, A.; Semikoz, D.; Taylor, A.M. Very hard gamma-ray emission from a flare of Mrk 501. Astron. Astrophys. 2012, 541, A31. [CrossRef]

47. Shukla, A.; Mannheim, K.; Chitnis, V.R.; Roy, J.; Acharya, B.S.; Dorner, D.; Hughes, G.; Biland, A. Detection of Very Hard $\gamma$-Ray Spectrum from the TeV Blazar Mrk 501. Astrophys. J. 2016, 832, 177. [CrossRef]

48. Rieger, F.M.; Aharonian, F.A. Centaurus A as $\mathrm{TeV} \gamma$-ray and possible UHE cosmic-ray source. Astron. Astrophys. 2009, 506, L41-L44. [CrossRef]

49. Rieger, F.M. Nonthermal Processes in Black Hole-Jet Magnetospheres. Int. J. Mod. Phys. D 2011, 20, 1547-1596. [CrossRef]

50. Ghisellini, G.; Tavecchio, F.; Chiaberge, M. Structured jets in TeV BL Lac objects and radiogalaxies. Implications for the observed properties. Astron. Astrophys. 2005, 432, 401-410, [CrossRef]

51. Lenain, J.P.; Boisson, C.; Sol, H.; Katarzyński, K. A synchrotron self-Compton scenario for the very high energy $\gamma$-ray emission of the radiogalaxy M 87. Unifying the TeV emission of blazars and other AGNs? Astron. Astrophys. 2008, 478, 111-120. [CrossRef]

52. Kachelrieß, M.; Ostapchenko, S.; Tomàs, R. TeV Gamma Rays from Ultrahigh Energy Cosmic Ray Interactions in the Cores of Active Galactic Nuclei: Lessons from Centaurus A. Publ. Astron. Soc. Aust. 2010, 27, 482-489. [CrossRef]

53. Sahu, S.; Zhang, B.; Fraija, N. Hadronic-origin $\mathrm{TeV} \gamma$ rays and ultrahigh energy cosmic rays from Centaurus A. Phys. Rev. D 2012, 85, 043012. [CrossRef]

54. Petropoulou, M.; Lefa, E.; Dimitrakoudis, S.; Mastichiadis, A. One-zone synchrotron self-Compton model for the core emission of Centaurus A revisited. Astron. Astrophys. 2014, 562, A12. [CrossRef]

55. Reynoso, M.M.; Medina, M.C.; Romero, G.E. A lepto-hadronic model for high-energy emission from FR I radiogalaxies. Astron. Astrophys. 2011, 531, A30. [CrossRef]

56. Cerruti, M.; Zech, A.; Emery, G.; Guarin, D. Hadronic modeling of TeV AGN: Gammas and neutrinos. In Proceedings of the 6th International Symposium on High Energy Gamma-Ray Astronomy, Heidelberg, Germany, 11-15 July 2016; Volume 1792, p. 050027. [CrossRef]

57. Sitarek, J.; Bednarek, W. $\gamma$-rays from the IC $\mathrm{e}^{+/-}$pair cascade in the radiation field of an accretion disc: Application to Cen A. Mon. Not. R. Astron. Soc. 2010, 401, 1983-1988. [CrossRef]

58. Roustazadeh, P.; Böttcher, M. Very High Energy Gamma-ray-induced Pair Cascades in the Radiation Fields of Dust Tori of Active Galactic Nuclei: Application to Cen A. Astrophys. J. 2011, 728, 134. [CrossRef]

59. Stawarz, Ł.; Aharonian, F.; Wagner, S.; Ostrowski, M. Absorption of nuclear $\gamma$-rays on the starlight radiation in FR I sources: The case of Centaurus A. Mon. Not. R. Astron. Soc. 2006, 371, 1705-1716. [CrossRef]

60. Stawarz, Ł.; Sikora, M.; Ostrowski, M. High-Energy Gamma Rays from FR I Jets. Astrophys. J. 2003, 597, 186-201. [CrossRef]

61. Hardcastle, M.J.; Croston, J.H. Modelling TeV $\gamma$-ray emission from the kiloparsec-scale jets of Centaurus A and M87. Mon. Not. R. Astron. Soc. 2011, 415, 133-142. [CrossRef]

62. Rieger, F.M. Gamma-rays from non-blazar AGN. In Proceedings of the 6th International Symposium on High Energy Gamma-Ray Astronomy, Heidelberg, Germany, 11-15 July 2016; Volume 1792, p. 020008. [CrossRef] 
63. Sanchez, D.; Holler, M.; Taylor, A.; Rieger, F.; DeNaurois, M.; HESS Collaboration. Morphology study of a radio galaxy. In Proceedings of the TeVPA Conference, Berlin, Germany, 27-31 August 2018.

64. Aleksić, J.; Antonelli, L.A.; Antoranz, P.; Backes, M.; Barrio, J.A.; Bastieri, D.; Becerra González, J.; Bednarek, W.; Berdyugin, A.; Berger, K.; et al. Detection of Very High Energy $\gamma$-ray Emission from the Perseus Cluster Head-Tail Galaxy IC 310 by the MAGIC Telescopes. Astrophys. J. 2010, 723, L207-L212. [CrossRef]

65. Aleksić, J.; Ansoldi, S.; Antonelli, L.A.; Antoranz, P.; Babic, A.; Bangale, P.; Barrio, J.A.; González, J.B.; Bednarek, W.; Bernardini, E.; et al. Black hole lightning due to particle acceleration at subhorizon scales. Science 2014, 346, 1080-1084. [CrossRef] [PubMed]

66. Ahnen, M.L.; Ansoldi, S.; Antonelli, L.A.; Arcaro, C.; Babić, A.; Banerjee, B.; Bangale, P.; Barres de Almeida, U.; Barrio, J.A.; Becerra González, J.; et al. First multi-wavelength campaign on the gamma-ray-loud active galaxy IC 310. Astron. Astrophys. 2017, 603, A25. [CrossRef]

67. Berton, M.; Foschini, L.; Ciroi, S.; Cracco, V.; La Mura, G.; Lister, M.L.; Mathur, S.; Peterson, B.M.; Richards, J.L.; Rafanelli, P. Parent population of flat-spectrum radio-loud narrow-line Seyfert 1 galaxies. Astron. Astrophys. 2015, 578, A28. [CrossRef]

68. Kadler, M.; Eisenacher, D.; Ros, E.; Mannheim, K.; Elsässer, D.; Bach, U. The blazar-like radio structure of the TeV source IC 310. Astron. Astrophys. 2012, 538, L1. [CrossRef]

69. Giannios, D. Reconnection-driven plasmoids in blazars: Fast flares on a slow envelope. Mon. Not. R. Astron. Soc. 2013, 431, 355-363. [CrossRef]

70. Barkov, M.V.; Aharonian, F.A.; Bogovalov, S.V.; Kelner, S.R.; Khangulyan, D. Rapid TeV Variability in Blazars as a Result of Jet-Star Interaction. Astrophys. J. 2012, 749, 119. [CrossRef]

71. Aharonian, F.A.; Barkov, M.V.; Khangulyan, D. Scenarios for Ultrafast Gamma-Ray Variability in AGN. Astrophys. J. 2017, 841, 61. [CrossRef]

72. Hirotani, K.; Pu, H.Y. Energetic Gamma Radiation from Rapidly Rotating Black Holes. Astrophys. J. 2016, 818, 50. [CrossRef]

73. Sijbring, D.; de Bruyn, A.G. Multifrequency radio continuum observations of head-tail galaxies in the Perseus cluster. Astron. Astrophys. 1998, 331, 901-915.

74. Aharonian, F.; Akhperjanian, A.; Beilicke, M.; Bernlöhr, K.; Börst, H.G.; Bojahr, H.; Bolz, O.; Coarasa, T.; Contreras, J.L.; Cortina, J.; et al. Is the giant radio galaxy M 87 a TeV gamma-ray emitter? Astron. Astrophys. 2003, 403, L1-L5. [CrossRef]

75. Walsh, J.L.; Barth, A.J.; Ho, L.C.; Sarzi, M. The M87 Black Hole Mass from Gas-dynamical Models of Space Telescope Imaging Spectrograph Observations. Astrophys. J. 2013, 770, 86. [CrossRef]

76. Reynolds, C.S.; Di Matteo, T.; Fabian, A.C.; Hwang, U.; Canizares, C.R. The 'quiescent' black hole in M87. Mon. Not. R. Astron. Soc. 1996, 283, L111-L116. [CrossRef]

77. Bird, S.; Harris, W.E.; Blakeslee, J.P.; Flynn, C. The inner halo of M 87: A first direct view of the red-giant population. Astron. Astrophys. 2010, 524, A71. [CrossRef]

78. Doeleman, S.S.; Fish, V.L.; Schenck, D.E.; Beaudoin, C.; Blundell, R.; Bower, G.C.; Broderick, A.E.; Chamberlin, R.; Freund, R.; Friberg, P.; et al. Jet-Launching Structure Resolved Near the Supermassive Black Hole in M87. Science 2012, 338, 355. [CrossRef] [PubMed]

79. Kino, M.; Takahara, F.; Hada, K.; Akiyama, K.; Nagai, H.; Sohn, B.W. Magnetization Degree at the Jet Base of M87 Derived from the Event Horizon Telescope Data: Testing the Magnetically Driven Jet Paradigm. Astrophys. J. 2015, 803, 30. [CrossRef]

80. Akiyama, K.; Lu, R.S.; Fish, V.L.; Doeleman, S.S.; Broderick, A.E.; Dexter, J.; Hada, K.; Kino, M.; Nagai, H.; Honma, M.; et al. 230 GHz VLBI Observations of M87: Event-horizon-scale Structure during an Enhanced Very-high-energy $\gamma$-Ray State in 2012. Astrophys. J. 2015, 807, 150. [CrossRef]

81. Hada, K.; Kino, M.; Doi, A.; Nagai, H.; Honma, M.; Akiyama, K.; Tazaki, F.; Lico, R.; Giroletti, M.; Giovannini, G.; et al. High-sensitivity $86 \mathrm{GHz}(3.5 \mathrm{~mm})$ VLBI Observations of M87: Deep Imaging of the Jet Base at a Resolution of 10 Schwarzschild Radii. Astrophys. J. 2016, 817, 131. [CrossRef]

82. Mertens, F.; Lobanov, A.P.; Walker, R.C.; Hardee, P.E. Kinematics of the jet in M 87 on scales of 100-1000 Schwarzschild radii. Astron. Astrophys. 2016, 595, A54. [CrossRef]

83. Globus, N.; Levinson, A. The collimation of magnetic jets by disc winds. Mon. Not. R. Astron. Soc. 2016, 461, 2605-2615. [CrossRef]

84. Rieger, F.M.; Aharonian, F. Probing the Central Black Hole in M87 with Gamma-Rays. Mod. Phys. Lett. A 2012, 27, 1230030. [CrossRef] 
85. Aharonian, F.; Akhperjanian, A.G.; Bazer-Bachi, A.R.; Beilicke, M.; Benbow, W.; Berge, D.; Bernlöhr, K.; Boisson, C.; Bolz, O.; Borrel, V.; et al. Fast Variability of Tera-Electron Volt $\gamma$ Rays from the Radio Galaxy M87. Science 2006, 314, 1424-1427. [CrossRef] [PubMed]

86. Albert, J.; Aliu, E.; Anderhub, H.; Antonelli, L.A.; Antoranz, P.; Backes, M.; Baixeras, C.; Barrio, J.A.; Bartko, H.; Bastieri, D.; et al. Very High Energy Gamma-Ray Observations of Strong Flaring Activity in M87 in 2008 February. Astrophys. J. 2008, 685, L23. [CrossRef]

87. Acciari, V.A.; Aliu, E.; Arlen, T.; Bautista, M.; Beilicke, M.; Benbow, W.; Bradbury, S.M.; Buckley, J.H.; Bugaev, V.; Butt, Y.; et al. Radio Imaging of the Very-High-Energy $\gamma$-Ray Emission Region in the Central Engine of a Radio Galaxy. Science 2009, 325, 444. [CrossRef] [PubMed]

88. Aliu, E.; Arlen, T.; Aune, T.; Beilicke, M.; Benbow, W.; Bouvier, A.; Bradbury, S.M.; Buckley, J.H.; Bugaev, V.; Byrum, K.; et al. VERITAS Observations of Day-scale Flaring of M 87 in 2010 April. Astrophys. J. 2012, 746, 141. [CrossRef]

89. Abramowski, A.; Acero, F.; Aharonian, F.; Akhperjanian, A.G.; Anton, G.; Balzer, A.; Barnacka, A.; Barres de Almeida, U.; Becherini, Y.; Becker, J.; et al. The 2010 Very High Energy $\gamma$-Ray Flare and 10 Years of Multi-wavelength Observations of M 87. Astrophys. J. 2012, 746, 151. [CrossRef]

90. Abdo, A.A.; Ackermann, M.; Ajello, M.; Atwood, W.B.; Axelsson, M.; Baldini, L.; Ballet, J.; Barbiellini, G.; Bastieri, D.; Bechtol, K.; et al. Fermi Large Area Telescope Gamma-Ray Detection of the Radio Galaxy M87. Astrophys. J. 2009, 707, 55-60. [CrossRef]

91. Acero, F.; Ackermann, M.; Ajello, M.; Albert, A.; Atwood, W.B.; Axelsson, M.; Baldini, L.; Ballet, J.; Barbiellini, G.; Bastieri, D.; et al. Fermi Large Area Telescope Third Source Catalog. Astrophys. J. Suppl. Ser. 2015, $218,23$. [CrossRef]

92. Ait Benkhali, F.; Chakraborty, N.; Rieger, F.M. The complex gamma-ray behaviour of the Radio Galaxy M87. arXiv 2018, arXiv:1802.03103.

93. Tavecchio, F.; Ghisellini, G. Spine-sheath layer radiative interplay in subparsec-scale jets and the $\mathrm{TeV}$ emission from M87. Mon. Not. R. Astron. Soc. 2008, 385, L98-L102. [CrossRef]

94. Giannios, D.; Uzdensky, D.A.; Begelman, M.C. Fast TeV variability from misaligned minijets in the jet of M87. Mon. Not. R. Astron. Soc. 2010, 402, 1649-1656. [CrossRef]

95. Reimer, A.; Protheroe, R.J.; Donea, A.C. M87 as a misaligned synchrotron-proton blazar. Astron. Astrophys. 2004, 419, 89-98. [CrossRef]

96. Georganopoulos, M.; Perlman, E.S.; Kazanas, D. Is the Core of M87 the Source of Its TeV Emission? Implications for Unified Schemes. Astrophys. J. 2005, 634, L33-L36. [CrossRef]

97. Rieger, F.M.; Aharonian, F.A. Variable VHE gamma-ray emission from non-blazar AGNs. Astron. Astrophys. 2008, 479, L5-L8. [CrossRef]

98. Levinson, A.; Rieger, F. Variable TeV Emission as a Manifestation of Jet Formation in M87? Astrophys. J. 2011, 730, 123. [CrossRef]

99. Rieger, F.M. On the origin of very high energy $\gamma$-rays from radio galaxies. In Proceedings of the 5th International Symposium on High-Energy Gamma-Ray Astronomy, Heidelberg, Germany, 9-13 July 2012; Volume 1505, pp. 80-87. [CrossRef]

100. Beilicke, M.; VERITAS Collaboration. VERITAS observations of M87 in 2011/2012. In Proceedings of the 5th International Symposium on High-Energy Gamma-Ray Astronomy, Heidelberg, Germany, 9-13 July 2012; Volume 1505, pp. 586-589. [CrossRef]

101. Bangale, P.; Manganaro, M.; Schultz, C.; Colin, P.; Mazin, D. Long term variability study for the radio galaxy M87 with MAGIC. In Proceedings of the 34th International Cosmic Ray Conference (ICRC2015), The Hague, The Netherlands, 30 July-6 August 2015; Volume 34, p. 759.

102. Hada, K.; Kino, M.; Nagai, H.; Doi, A.; Hagiwara, Y.; Honma, M.; Giroletti, M.; Giovannini, G.; Kawaguchi, N. VLBI Observations of the Jet in M 87 during the Very High Energy $\gamma$-Ray Flare in 2010 April. Astrophys. J. 2012, 760, 52. [CrossRef]

103. Hada, K.; Giroletti, M.; Kino, M.; Giovannini, G.; D’Ammando, F.; Cheung, C.C.; Beilicke, M.; Nagai, H.; Doi, A.; Akiyama, K.; et al. A Strong Radio Brightening at the Jet Base of M 87 during the Elevated Very High Energy Gamma-Ray State in 2012. Astrophys. J. 2014, 788, 165. [CrossRef]

104. Broderick, A.E.; Tchekhovskoy, A. Horizon-scale Lepton Acceleration in Jets: Explaining the Compact Radio Emission in M87. Astrophys. J. 2015, 809, 97. [CrossRef] 
105. Ptitsyna, K.; Neronov, A. Particle acceleration in the vacuum gaps in black hole magnetospheres. Astron. Astrophys. 2016, 593, A8. [CrossRef]

106. Blandford, R.D.; Znajek, R.L. Electromagnetic extraction of energy from Kerr black holes. Mon. Not. R. Astron. Soc. 1977, 179, 433-456. [CrossRef]

107. Beskin, V.S.; Istomin, Y.N.; Parev, V.I. Filling the Magnetosphere of a Supermassive Black-Hole with Plasma. Sov. Astron. 1992, 36, 642.

108. Hirotani, K.; Okamoto, I. Pair Plasma Production in a Force-free Magnetosphere around a Supermassive Black Hole. Astrophys. J. 1998, 497, 563-572. [CrossRef]

109. Levinson, A. Particle Acceleration and Curvature TeV Emission by Rotating, Supermassive Black Holes. Phys. Rev. Lett. 2000, 85, 912-915. [CrossRef] [PubMed]

110. Neronov, A.; Aharonian, F.A. Production of TeV Gamma Radiation in the Vicinity of the Supermassive Black Hole in the Giant Radio Galaxy M87. Astrophys. J. 2007, 671, 85-96. [CrossRef]

111. Hirotani, K.; Pu, H.Y.; Lin, L.C.C.; Chang, H.K.; Inoue, M.; Kong, A.K.H.; Matsushita, S.; Tam, P.H.T. Lepton Acceleration in the Vicinity of the Event Horizon: High-energy and Very-high-energy Emissions from Rotating Black Holes with Various Masses. Astrophys. J. 2016, 833, 142. [CrossRef]

112. Lin, L.C.C.; Pu, H.Y.; Hirotani, K.; Kong, A.K.H.; Matsushita, S.; Chang, H.K.; Inoue, M.; Tam, P.H.T. Searching for High-energy, Horizon-scale Emissions from Galactic Black Hole Transients during Quiescence. Astrophys. J. 2017, 845, 40. [CrossRef]

113. Levinson, A.; Segev, N. Existence of steady gap solutions in rotating black hole magnetospheres. Phys. Rev. D 2017, 96, 123006. [CrossRef]

114. Hirotani, K.; Pu, H.Y.; Lin, L.C.C.; Kong, A.K.H.; Matsushita, S.; Asada, K.; Chang, H.K.; Tam, P.H.T. Lepton Acceleration in the Vicinity of the Event Horizon: Very High Energy Emissions from Supermassive Black Holes. Astrophys. J. 2017, 845, 77. [CrossRef]

115. Globus, N.; Levinson, A. Jet Formation in GRBs: A Semi-analytic Model of MHD Flow in Kerr Geometry with Realistic Plasma Injection. Astrophys. J. 2014, 796, 26. [CrossRef]

116. Levinson, A.; Cerutti, B. Particle-in-cell simulations of pair discharges in a starved magnetosphere of a Kerr black hole. Astron. Astrophys. 2018, 616, A184. [CrossRef]

117. Chen, A.Y.; Yuan, Y.; Yang, H. Physics of Pair Producing Gaps in Black Hole Magnetospheres. Astrophys. J. 2018, 863, L31. [CrossRef]

118. Levinson, A.; Melrose, D.; Judge, A.; Luo, Q. Large-Amplitude, Pair-creating Oscillations in Pulsar and Black Hole Magnetospheres. Astrophys. J. 2005, 631, 456-465. [CrossRef]

119. Chiaberge, M.; Celotti, A.; Capetti, A.; Ghisellini, G. Does the unification of BL Lac and FR I radio galaxies require jet velocity structures? Astron. Astrophys. 2000, 358, 104-112.

120. Meyer, E.T.; Fossati, G.; Georganopoulos, M.; Lister, M.L. From the Blazar Sequence to the Blazar Envelope: Revisiting the Relativistic Jet Dichotomy in Radio-loud Active Galactic Nuclei. Astrophys. J. 2011, 740, 98. [CrossRef]

121. Sbarrato, T.; Padovani, P.; Ghisellini, G. The jet-disc connection in AGN. Mon. Not. R. Astron. Soc. 2014, 445, 81-92. [CrossRef]

122. Rieger, F.M.; Duffy, P. Shear Acceleration in Relativistic Astrophysical Jets. Astrophys. J. 2004, 617, $155-161$. [CrossRef]

123. Tavecchio, F.; Ghisellini, G. On the magnetization of BL Lac jets. Mon. Not. R. Astron. Soc. 2016, 456, $2374-2382$. [CrossRef]

124. Giannios, D.; Uzdensky, D.A.; Begelman, M.C. Fast TeV variability in blazars: Jets in a jet. Mon. Not. R. Astron. Soc. 2009, 395, L29-L33. [CrossRef]

125. Nalewajko, K.; Giannios, D.; Begelman, M.C.; Uzdensky, D.A.; Sikora, M. Radiative properties of reconnection-powered minijets in blazars. Mon. Not. R. Astron. Soc. 2011, 413, 333-346. [CrossRef]

126. Cerutti, B.; Werner, G.R.; Uzdensky, D.A.; Begelman, M.C. Beaming and Rapid Variability of High-energy Radiation from Relativistic Pair Plasma Reconnection. Astrophys. J. 2012, 754, L33. [CrossRef]

127. Kadowaki, L.H.S.; de Gouveia Dal Pino, E.M.; Singh, C.B. The Role of Fast Magnetic Reconnection on the Radio and Gamma-ray Emission from the Nuclear Regions of Microquasars and Low Luminosity AGNs. Astrophys. J. 2015, 802, 113. [CrossRef]

128. Sironi, L.; Petropoulou, M.; Giannios, D. Relativistic jets shine through shocks or magnetic reconnection? Mon. Not. R. Astron. Soc. 2015, 450, 183-191. [CrossRef] 
129. Werner, G.R.; Uzdensky, D.A.; Cerutti, B.; Nalewajko, K.; Begelman, M.C. The Extent of Power-law Energy Spectra in Collisionless Relativistic Magnetic Reconnection in Pair Plasmas. Astrophys. J. 2016, 816, L8. [CrossRef]

130. Mizuno, Y.; Lyubarsky, Y.; Nishikawa, K.I.; Hardee, P.E. Three-dimensional Relativistic Magnetohydrodynamic Simulations of Current-driven Instability. III. Rotating Relativistic Jets. Astrophys. J. 2012, 757, 16. [CrossRef]

131. O'Neill, S.M.; Beckwith, K.; Begelman, M.C. Local simulations of instabilities in relativistic jets-I. Morphology and energetics of the current-driven instability. Mon. Not. R. Astron. Soc. 2012, 422, 1436-1452. [CrossRef]

132. Guan, X.; Li, H.; Li, S. Relativistic MHD Simulations of Poynting Flux-driven Jets. Astrophys. J. 2014, 781, 48. [CrossRef]

133. Parfrey, K.; Giannios, D.; Beloborodov, A.M. Black hole jets without large-scale net magnetic flux. Mon. Not. R. Astron. Soc. 2015, 446, L61-L65. [CrossRef]

134. Levinson, A.; Globus, N. The effect of Compton drag on the dynamics of dissipative Poynting-dominated flows: Implications for the unification of radio loud AGN. Mon. Not. R. Astron. Soc. 2016, 458, 2269-2274. [CrossRef]

135. Cui, Y.D.; Yuan, Y.F.; Li, Y.R.; Wang, J.M. A General Relativistic External Compton-Scattering Model for TeV Emission from M87. Astrophys. J. 2012, 746, 177. [CrossRef]

136. Lyubarsky, Y.E. On the relativistic magnetic reconnection. Mon. Not. R. Astron. Soc. 2005, 358, $113-119$. [CrossRef]

137. Lemoine, M.; Pelletier, G. Particle acceleration at relativistic shock waves. In Proceedings of the 6th International Symposium on High Energy Gamma-Ray Astronomy, Heidelberg, Germany, 11-15 July 2016; Volume 1792, p. 020006. [CrossRef]

138. Zenitani, S.; Hoshino, M. The Generation of Nonthermal Particles in the Relativistic Magnetic Reconnection of Pair Plasmas. Astrophys. J. 2001, 562, L63-L66. [CrossRef]

139. Guo, F.; Liu, Y.H.; Daughton, W.; Li, H. Particle Acceleration and Plasma Dynamics during Magnetic Reconnection in the Magnetically Dominated Regime. Astrophys. J. 2015, 806, 167. [CrossRef]

140. Sironi, L.; Giannios, D.; Petropoulou, M. Plasmoids in relativistic reconnection, from birth to adulthood: First they grow, then they go. Mon. Not. R. Astron. Soc. 2016, 462, 48-74. [CrossRef]

141. Petropoulou, M.; Christie, I.M.; Sironi, L.; Giannios, D. Plasmoid statistics in relativistic magnetic reconnection. Mon. Not. R. Astron. Soc. 2018, 475, 3797-3812. [CrossRef]

142. Perucho, M.; Bosch-Ramon, V.; Barkov, M.V. Impact of red giant/AGB winds on active galactic nucleus jet propagation. Astron. Astrophys. 2017, 606, A40. [CrossRef]

143. Wykes, S.; Hardcastle, M.J.; Karakas, A.I.; Vink, J.S. Internal entrainment and the origin of jet-related broad-band emission in Centaurus A. Mon. Not. R. Astron. Soc. 2015, 447, 1001-1013. [CrossRef]

144. Barkov, M.V.; Aharonian, F.A.; Bosch-Ramon, V. Gamma-ray Flares from Red Giant/Jet Interactions in Active Galactic Nuclei. Astrophys. J. 2010, 724, 1517-1523. [CrossRef]

145. Bosch-Ramon, V.; Perucho, M.; Barkov, M.V. Clouds and red giants interacting with the base of AGN jets. Astron. Astrophys. 2012, 539, A69. [CrossRef]

146. Araudo, A.T.; Bosch-Ramon, V.; Romero, G.E. Gamma-ray emission from massive stars interacting with active galactic nuclei jets. Mon. Not. R. Astron. Soc. 2013, 436, 3626-3639. [CrossRef]

147. Khangulyan, D.V.; Barkov, M.V.; Bosch-Ramon, V.; Aharonian, F.A.; Dorodnitsyn, A.V. Star-Jet Interactions and Gamma-Ray Outbursts from 3C454.3. Astrophys. J. 2013, 774, 113. [CrossRef]

148. Bednarek, W.; Banasiński, P. Non-thermal Radiation from Collisions of Compact Objects with Intermediate-scale Jets in Active Galaxies. Astrophys. J. 2015, 807, 168. [CrossRef]

149. Bosch-Ramon, V. Non-thermal emission from standing relativistic shocks: An application to red giant winds interacting with AGN jets. Astron. Astrophys. 2015, 575, A109. [CrossRef]

150. De la Cita, V.M.; Bosch-Ramon, V.; Paredes-Fortuny, X.; Khangulyan, D.; Perucho, M. Coupling hydrodynamics and radiation calculations for star-jet interactions in active galactic nuclei. Astron. Astrophys. 2016, 591, A15. [CrossRef]

151. Zacharias, M.; Böttcher, M.; Jankowsky, F.; Lenain, J.P.; Wagner, S.J.; Wierzcholska, A. Cloud Ablation by a Relativistic Jet and the Extended Flare in CTA 102 in 2016 and 2017. Astrophys. J. 2017, 851, 72. [CrossRef]

152. Vieyro, F.L.; Torres-Albà, N.; Bosch-Ramon, V. Collective non-thermal emission from an extragalactic jet interacting with stars. Astron. Astrophys. 2017, 604, A57. [CrossRef] 
153. Barkov, M.V.; Bosch-Ramon, V.; Aharonian, F.A. Interpretation of the Flares of M87 at TeV Energies in the Cloud-Jet Interaction Scenario. Astrophys. J. 2012, 755, 170. [CrossRef]

154. Harris, D.E.; Krawczynski, H. X-Ray Emission from Extragalactic Jets. Annu. Rev. Astron. Astrophys. 2006, 44, 463-506. [CrossRef]

155. Georganopoulos, M.; Meyer, E.; Perlman, E. Recent Progress in Understanding the Large Scale Jets of Powerful Quasars. Galaxies 2016, 4, 65. [CrossRef]

156. Liu, R.Y.; Rieger, F.M.; Aharonian, F.A. Particle Acceleration in Mildly Relativistic Shearing Flows: The Interplay of Systematic and Stochastic Effects, and the Origin of the Extended High-energy Emission in AGN Jets. Astrophys. J. 2017, 842, 39. [CrossRef]

157. Sun, X.N.; Yang, R.Z.; Rieger, F.M.; Liu, R.Y.; Aharonian, F. Energy distribution of relativistic electrons in the kiloparsec scale jet of M 87 with Chandra. Astron. Astrophys. 2018, 612, A106. [CrossRef]

158. Cherenkov Telescope Array Consortium; Acharya, B.S.; Agudo, I.; Samarai, I.A.; Alfaro, R.; Alfaro, J.; Alispach, C.; Alves Batista, R.; Amans, J.P.; Amato, E.; et al. Science with the Cherenkov Telescope Array. arXiv 2017, arXiv:1709.07997.

(C) 2018 by the authors. Licensee MDPI, Basel, Switzerland. This article is an open access article distributed under the terms and conditions of the Creative Commons Attribution (CC BY) license (http:/ / creativecommons.org/licenses/by/4.0/). 
Review

\title{
Very High-Energy Emission from the Direct Vicinity of Rapidly Rotating Black Holes
}

\author{
Kouichi Hirotani \\ Academia Sinica, Institute of Astronomy and Astrophysics, AS/NTU Astronomy-Mathematics Building, No. 1, \\ Sec. 4, Roosevelt Rd., Taipei 10617, Taiwan; hirotani@asiaa.sinica.edu.tw; Tel.: +886-2-3365-5406
}

Received: 31 October 2018; Accepted: 19 November 2018; Published: 22 November 2018

\begin{abstract}
When a black hole accretes plasmas at very low accretion rate, an advection-dominated accretion flow (ADAF) is formed. In an ADAF, relativistic electrons emit soft gamma-rays via Bremsstrahlung. Some $\mathrm{MeV}$ photons collide with each other to materialize as electron-positron pairs in the magnetosphere. Such pairs efficiently screen the electric field along the magnetic field lines, when the accretion rate is typically greater than $0.03-0.3 \%$ of the Eddington rate. However, when the accretion rate becomes smaller than this value, the number density of the created pairs becomes less than the rotationally induced Goldreich-Julian density. In such a charge-starved magnetosphere, an electric field arises along the magnetic field lines to accelerate charged leptons into ultra-relativistic energies, leading to an efficient $\mathrm{TeV}$ emission via an inverse-Compton (IC) process, spending a portion of the extracted hole's rotational energy. In this review, we summarize the stationary lepton accelerator models in black hole magnetospheres. We apply the model to super-massive black holes and demonstrate that nearby low-luminosity active galactic nuclei are capable of emitting detectable gamma-rays between 0.1 and $30 \mathrm{TeV}$ with the Cherenkov Telescope Array.
\end{abstract}

Keywords: gamma-rays: observation; gamma-rays: theory; general relativity; particle acceleration; stars: black holes

\section{Introduction}

It is commonly accepted that every active galaxy harbors a supermassive black hole (BH), whose mass typically ranges between $10^{6} \mathrm{M}_{\odot}$ and $10^{9.5} \mathrm{M}_{\odot}$, in its center (e.g., [1-4]; see also [5,6] for reviews). Compelling evidence of a supermassive $\mathrm{BH}$ was found by observing the line emission from water masers around the central region of galaxy NGC 4258 [7]. Moreover, evidence of supermassive $\mathrm{BHs}$ at individual galactic centers are shown by the tight correlation between the black hole mass and the velocity dispersion or the bulge mass (e.g., [8-10]), which has been confirmed for the measurements with reverberation mapping (e.g., [11]).

A likely mechanism for powering such an active galactic nucleus (AGN) is the release of the gravitational energy of accreting plasmas [12] or the electromagnetic extraction of the rotational energy of a rotating supermassive BH [13]. The latter mechanism, which is called the Blandford-Znajek (BZ) mechanism, works only when there is a plasma accretion, because a BH cannot have its own magnetic moment (e.g., [14]). As long as the magnetic field energy is in a rough equipartition with the gravitational binding energy of the accreting plasmas, both mechanisms contribute comparably in terms of luminosity. The former mechanism is supposed to power the mildly relativistic winds that are launched from the accretion disks [15-17]. There is, however, growing evidence that relativistic jets are energized by the latter BZ mechanism through numerical simulations [18-20] (see also [21] for an ergospheric disk jet model). Indeed, general relativistic (GR) magnetohydrodynamic (MHD) models show the existence of collimated and magnetically dominated jets in the polar regions [22-24], whose structures are similar to those in the force-free models [25-27]. Since the centrifugal-force 
barrier prevents plasma accretion toward the rotation axis, the magnetic energy density dominates the plasmas' rest-mass energy density in these polar funnels.

Within such a nearly vacuum, polar funnel, electron-positron pairs are supplied via the collisions of $\mathrm{MeV}$ photons emitted from the equatorial, accreting region. For example, when the mass accretion rate is typically less than $1 \%$ of the Eddington rate, the accreting plasmas form an advection-dominated accretion flow (ADAF), emitting radio to infrared photons via the synchrotron process and $\mathrm{MeV}$ photons via free-free and inverse-Compton (IC) processes [28,29]. Particularly, when the accretion rate becomes much less than the Eddington rate, the $\mathrm{ADAF} \mathrm{MeV}$ photons can no longer sustain a force-free magnetosphere, which inevitably leads to the appearance of an electric field, $E_{\|}$, along the magnetic field lines in the polar funnel. In such a vacuum gap, we can expect that the BZ power may be partially dissipated as particle acceleration and emission near the central engine, in the same manner as in pulsar outer gap (OG) model. In what follows, we summarize this vacuum gap model in BH magnetospheres.

\section{The Pulsar Outer Gap Model}

The Large Area Telescope (LAT) aboard the Fermi space gamma-ray observatory has detected pulsed signals in high-energy (HE) $(0.1 \mathrm{GeV}-10 \mathrm{GeV})$ gamma-rays from more than 200 rotation-powered pulsars [30]. Among them, 20 pulsars exhibit pulsed signals above $10 \mathrm{GeV}$, including 10 pulsars up to $25 \mathrm{GeV}$ and other 2 pulsars above $50 \mathrm{GeV}$. Moreover, more than $99 \%$ of the LAT-detected young and millisecond pulsars exhibit phase-averaged spectra that are consistent with a pure-exponential or a sub-exponential cut off above the cut-off energies at a few $\mathrm{GeV}$. What is more, $30 \%$ of these young pulsars show sub-exponential cut off, a slower decay than the pure-exponential functional form. These facts preclude the possibility of emissions from the inner magnetosphere as in the polar-cap scenario [31-35], which predicts super-exponential cut off due to magnetic attenuation. That is, we can conclude that the pulsed gamma-ray emissions are mainly emitted from the outer magnetosphere that is close to the light cylinder, whose distance from the rotation axis is given by the so-called special-relativistic "light cylinder radius", $c / \Omega_{F}$, where $c$ denotes the speed of light and $\mathrm{F}$ the angular frequency of magnetic field rotation.

In a pulsar magnetosphere, the rotational energy of the neutron star is extracted by the magnetic torque and transferred outward as a Poynting flux. Most of such extracted energy is dissipated at large distances such as in the pulsar wind nebula. However, a small portion (typically $0.1-20 \%$ ) can be dissipated as particle acceleration and resultant radiation in the outer magnetosphere, showing pulsed, incoherent emissions. Thus, the luminosity of a magnetospheric particle accelerator (i.e., a gap) does not exceed the neutron star's spin-down luminosity.

One of the main scenarios of such outer-magnetospheric emissions is the OG model [36-47]. In an $O G$, an electric field, $E_{\|}$, is exerted along the local magnetic field line near the null-charge surface, on which the rotationally induced Goldreich-Julian (GJ) charge density vanishes due to the convex geometry of the dipolar-like magnetic field of the neutron star. The $E_{\|}$accelerate electrons and positrons into ultra-relativistic energies, leading to the emission of HE photons typically between $10 \mathrm{MeV}$ and $10 \mathrm{GeV}$ via the synchro-curvature process [48,49]. Electrons and positrons (which are referred to as leptons in this review) are accelerated by the magnetic-field aligned electric field, $E_{\|}$, and saturate at Lorentz factors typically below $10^{7.5}$ due to the radiation drag of the synchro-curvature process. Because of a superposition of the synchro-curvature emission from different places in the gap, a power-law spectrum can be obtained below the cut off, which appears typically at a few $\mathrm{GeV}$ [50]. In addition, if the system becomes non-stationary, the linear acceleration may play an important role, provided that the acceleration takes place within a short length scale, $R_{\mathrm{c}} / \gamma$, where $R_{\mathrm{c}}$ denotes the curvature radius of the 3-D particle motion, and $\gamma$ the lepton Lorentz factor. In this case, the resulting spectrum will show a power-law energy dependence blow the cutoff [51], in the same manner as in the jitter radiation in the synchrotron process. 
This successful pulsar OG scenario was applied to BH magnetospheres, which will be described in the rest of this review.

\section{The Black Hole Gap Model}

\subsection{Stationary Gap Models}

Following the successful pulsar OG model, Beskin et al. applied it to BH magnetospheres for the first time [52]. It was shown that an efficient pair-production cascade can take place near the null-charge surface, where the GJ charge density vanishes due to the space-time frame-dragging around a rotating $\mathrm{BH}$. Then it was demonstrated that the stationary gap solutions can be obtained from the set of Maxwell-Boltzmann equations in a consistent manner with the gap closure condition [53]. However, they considered mass accretion rates that are a good fraction of the Eddington accretion rate. Thus, the solved gap width, $w$, along the magnetic field lines, became much less than the gravitational radius, $r_{\mathrm{g}}=G M / c^{2}$, where $M$ designates the $\mathrm{BH}$ mass, $c$ the speed of light, and $G$ the gravitational constant. In what follows, we adopt the geometrized unit, putting $c=G=1$. Because of the small gap width, $w \ll M$, the exerted $E_{\|}$is found to be very small compared to the magnetic-field strength, $B$, in a Gaussian unit; as a result, the gap luminosity, $L_{\mathrm{gap}}$, became negligibly small compared to the Eddington luminosity. For a pure hydrogen gas, the Eddington luminosity becomes $L_{\text {Edd }}=1.25 \times 10^{47} \mathrm{Mg}_{9} \mathrm{erg} \mathrm{s}^{-1}$, where $M_{9} \equiv M / 10^{9} M_{\odot}$.

On these grounds, to achieve bright gap emissions, we began to consider spatially extended gaps, which can be possible if the soft photon field is weak enough so that the photon-photon pair-production mean-free path becomes comparable to or greater than the gravitational radius, $r_{\mathrm{g}}=M$. For example, when the mass accretion rate is typically less than $1 \%$ of the Eddington rate, the accreting plasmas form an $\mathrm{ADAF}$, emitting radio to infrared photons via synchrotron process and $\mathrm{MeV}$ photons via free-free and IC processes $(\S 4)$. Under such a low accretion environment, gap-emitted TeV photons do not efficiently collide with the soft photons, leading to an un-screened, spatially extended gap, which has a much greater electric potential drop, and hence $L_{\text {gap }}$ than the denser soft-photon-field case.

In this context, Neronov and Aharonian [54] examined a $\mathrm{BH}$ gap emission and applied it to the central engine of M87, a nearby low luminosity AGN, which hosts a BH with mass $M \approx(3.2-6.6) \times 10^{9}$ $\mathrm{M}_{\odot}$ [55-58]. They assumed that the gap is extended, $w \approx 2 M$ (= Schwarzschild radius), that the lepton number density, $N_{ \pm}$, is comparable to the typical GJ number density, $N_{\mathrm{GJ}} \sim \Omega_{\mathrm{F}} B /(2 \pi c e)$, where $e$ denotes the magnitude of the charge on the electron, and that the magnetic field strength $B$ becomes comparable to the equipartition value,

$$
B_{e q} \approx 4 \times 10^{4} \dot{m}^{1 / 2} M_{9}{ }^{-1 / 2},
$$

which is obtained when the magnetic buoyancy balances the disk gravity; $\dot{m} \equiv \dot{M} / \dot{M}_{\mathrm{Edd}}$ refers to the dimensionless accretion rate near the horizon; and $\dot{M}$ denotes the mass accretion rate. We have $L_{\mathrm{Edd}}=\eta \dot{M}_{\mathrm{Edd}} c^{2}$, where the radiation efficiency can be estimated as $\eta \approx 0.1$. They also assumed that the magnetic-field-aligned electric field is comparable to the perpendicular component,

$$
E_{\|} \approx E_{\perp} \approx \frac{\omega\left(\Omega_{\mathrm{F}}-\omega\right)}{c} B \approx \frac{a B_{\mathrm{H}}}{2 M},
$$

where this denotes the distance from the rotation axis, with the spacetime dragging angular frequency due to $\mathrm{BH}^{\prime}$ s rotation, $B_{\mathrm{H}}$ does the magnetic field strength evaluated at the horizon. In the right-most near equality, we evaluate quantities near the horizon, where a gap is formed. Because of these four assumptions (i.e., $w \approx 2 M, N_{ \pm} \approx N_{\mathrm{GJ}}, B \approx B_{\mathrm{eq}}, E_{\|} \approx E_{\perp}$ ), $L_{\text {gap }}$ becomes comparable to the BZ power, $L_{\mathrm{BZ}}$, which corresponds to the spin-down luminosity of pulsars. Under these assumptions, they demonstrated that the observed very-high-energy (VHE) emission of M87 can be explained as the 
IC emission of ultra-relativistic leptons accelerated in a BH gap. See Section 7.1 for a brief summary of the VHE observations of M87 and other a few non-blazer AGNs.

Subsequently, Levinson and Rieger applied the vacuum gap model to M87 and found that the lepton density is strongly dependent on the accretion rate [59],

$$
N_{ \pm}=3 \times 10^{11} \dot{m}^{4} M_{9}^{-1} \mathrm{~cm}^{-3},
$$

and that $L_{\text {gap }} \approx L_{\mathrm{BZ}}$ holds if $w$ is a good fraction of $2 M$. Considering the ADAF photon field near the $\mathrm{BH}$, and assuming $w>0.1 \times 2 M, N_{ \pm} \approx N_{\mathrm{GJ}}, B \approx B_{\mathrm{eq}}$ and $E_{\|} \approx(w / 2 M)^{2} E_{\perp}$, they demonstrated that the VHE luminosity observed with Imaging Atmospheric Cherenkov Telescopes (IACTs) (7.1) can be reproduced by the $\mathrm{BH}$ gap model. They also argued that the gap activity may be intermittent, because the strong dependence of $N_{ \pm}$on $\dot{m}$ could lead to the disappearance of the gap due to moderate changes in the accretion rate.

Then Brodrick and Tchekhovskoy [60] considered a thick gap, $w \approx 2 M$, and an equipartition magnetic field strength, $B \approx B_{\text {eq, }}$, but adopted $N_{ \pm} \approx 10^{5} N_{\mathrm{GJ}}$ and $E_{\|} \gg E_{\perp}$. They showed that the stagnation surface $(\S 5.3)$ is a natural site of gap formation.

More recently, Hirotani and $\mathrm{Pu}$ [61] considered a one-dimensional BH gap, solving $w, N_{ \pm}$, and $E_{\|}$ from the set of the inhomogeneous part of the Maxwell equations (i.e., the Poisson equation) for the non-corotational potential, lepton density and velocity at each position, and the radiative transfer equation (i.e., instead of assuming $w, N_{ \pm}$, and $E_{\|}$, adopting $B \approx B_{\text {eq }}$. Then Hirotani et al. (2016) solved the same set of Maxwell-Boltzmann equations in the two-dimensional (2D) poloidal plane, assuming a mono-energetic approximation for the lepton distribution functions [62]. Subsequently, Hirotani et al. (2017) considered an inhomogeneous soft photon field to examine the $\gamma$-ray emission properties of supermassive BHs, solving the distribution functions of the accelerated leptons explicitly from their Boltzmann equations, discarding the mono-energetic approximation [63]. They showed that the accelerated leptons emit copious photons via IC processes between 0.1 and $30 \mathrm{TeV}$ for a distant observer, and that these IC fluxes will be detectable with IACTs such as the Cherenkov Telescope Array (CTA), provided that a low-luminosity active galactic nucleus is located within $1 \mathrm{Mpc}$ for a million-solar-mass central $\mathrm{BH}$ or within $30 \mathrm{Mpc}$ for a billion-solar-mass central $\mathrm{BH}$. Lin et al. then applied this method to stellar-mass BHs and compare the prediction with the high-energy (HE) observations, re-analyzing the archival Fermi/LAT data [64]. In addition, Song et al. demonstrated that the gap emission of an aligned rotator is enhanced along the rotation axis if the $\mathrm{BH}$ is nearly maximally rotating (i.e., $a \rightarrow M$ ) [65], because the magnetic fluxes concentrate polewards as $a \rightarrow M$ due to the magnetic pinch effect $[24,66]$. More recently, Hirotani et al. $(2018 a, b)$ investigated if stellar-mass BHs can emit detectable HE and VHE emissions when they encounter dense molecular clouds $(6)[67,68]$. In these stationary analysis $[61-65,67,68]$, They found $L_{\text {gap }} \approx\left(10^{-1}-10^{-4}\right) L_{\mathrm{BZ}}$, depending on the accretion rate. The BZ power becomes [13,23]:

$$
L_{B Z}=\Omega_{F}\left(\omega_{H}-\Omega_{F}\right) B_{\perp}{ }^{2} r_{H}{ }^{4},
$$

where $\omega_{\mathrm{H}}$ denotes the BH's rotational angular frequency, $r_{\mathrm{H}}$ does the horizon radius, and $B_{\perp}$ does the strength of the magnetic field component perpendicular to the horizon. The BZ power maximizes when $\Omega_{F}=0.5 \omega_{H}$. For a super-massive $B H$, the BZ power takes the typical value, $L_{B Z} \approx 10^{45}(a / M)^{2} B_{4}{ }^{2} M_{9}{ }^{2}$ erg/s, where $B_{4} \equiv B / 10^{4} G$, and $a / M$ denotes the dimensionless $B H$ spin, which vanishes for Schwarzschild BHs and becomes 1 for maximally rotating Kerr BHs. Exactly speaking, Equation (4) is obtained in the slow rotating limit, $a \ll M$ to the second order, $(a / M)^{2}$. For rapidly rotating BHs, the BZ power is obtained by Tanabe and Nagataki [69] to the fourth order, and by Tchekhovskoy et al. [24] to the sixth order. The higher-order corrections suppress the BZ power and a flattening occurs as a function of $(a / M)^{2}$ for extremely rotating BHs: for example, Equation (4) over-predicts the BZ power when $a>0.95 M$ for geometrically thin disks. 


\subsection{Force-Free Magnetosphere and the Necessity of a Gap}

For the BZ process to work, there should exist a global electric current in the magnetosphere. However, plasmas flow inwards inside the inner light surface and flow outwards outside the outer light surface (e.g., [70]). Thus, plasmas should be continuously replenished between the two light surfaces (see $\S 5.2$ for details) so that the BZ process may continuously work. If the created pair density, $N_{ \pm}$, exceeds $N_{\mathrm{GJ}}, E_{\|}$will be quickly screened out by the charge redistribution and the magnetosphere is kept force-free. For $N_{ \pm}>N_{\mathrm{G}}$ to be realized, the soft photon density should be large enough near the event horizon. In the case of an ADAF, the density of the pairs created via the collisions of the ADAF-emitted MeV photons, becomes [59]:

$$
N_{ \pm}=3 \times 10^{11} \dot{m}^{4} M_{9}^{-1} \mathrm{~cm}^{-3} \text {. }
$$

Comparing with $N_{\mathrm{GJ}} \sim \Omega_{\mathrm{F}} B /(2 \pi c e)$, we find [59]:

$$
\frac{N_{ \pm}}{N_{\mathrm{GJ}}}=0.06\left(\frac{\dot{m}}{10^{-4}}\right)^{7 / 2} M_{9}^{1 / 2}
$$

Thus, if the accretion satisfies:

$$
\dot{m}>2.2 \times 10^{-4} \mathrm{M}_{9}^{-1 / 7}
$$

the magnetosphere is kept force-free. In this case, there appears no gap. On the other hand, if the accretion rate stays below this value, the charge-starved magnetosphere inevitably has a gap, which may be either stationary or non-stationary.

Indeed, there is a growing consensus that the radio polarimetry in millimeter-submillimeter wavelengths provides useful diagnostics to infer the accretion rate of ADAF near the central engine. Through the Faraday rotation measure of the linear polarization, it is suggested that the dimensionless accretion rate becomes $\dot{m}=(1-10) \times 10^{-6}$ within the radius $r<200 M$ (=100 Schwarzschild radii) for Sgr $A^{*}$ [71-73] and $\dot{m}<10^{-4}$ within $r<42 M$ for M87 [74]. In addition, the jet luminosity $\left(\sim 2 \times 10^{42} \mathrm{ergs} \mathrm{s}^{-1}\right)$ of radio galaxy IC 310 [75] shows that the time-averaged accretion rate is $\dot{m} \sim 10^{-4}$ [76]. For low luminosity AGNs like M87 and IC 310 to sustain large-scale jets under such small accretion rates, sufficient pair creation should be taking place by the other methods than the collisions of ADAF-emitted $\mathrm{MeV}$ photons. As demonstrated in previous $\mathrm{BH}$ gap models, $\mathrm{BH}$ gaps are indeed capable of supplying such plasmas in a charge-starved magnetosphere via the cascade of the gap-emitted $\mathrm{TeV}$ photons into electron-positron pairs.

\subsection{Detectability of Gap Emissions}

As we have just seen, when the accretion rate becomes as small as:

$$
\dot{m}<\dot{m}_{\mathrm{up}}=2.2 \times 10^{-4} \mathrm{M}_{9}{ }^{-1 / 7}
$$

the gap is 'switched on' and gamma rays are emitted by the gap-accelerated lepton. Let us examine if we can detect such gamma-rays. First, to constrain the range of accretion rate in which a $\mathrm{BH}$ gap can be activated, we plot the upper limit accretion rate, $\dot{m}_{\mathrm{up}}=\dot{m}_{\mathrm{up}}(M)$ (Equation (8)) as the thick solid line in Figure 1. Above this upper limit, the magnetosphere becomes force-free (blue shaded region). Thus, only under this thick solid line, $\mathrm{BH}$ gaps can be formed (white region). The dotted lines denote constant- $L_{\mathrm{BZ}}$ lines as labeled. 


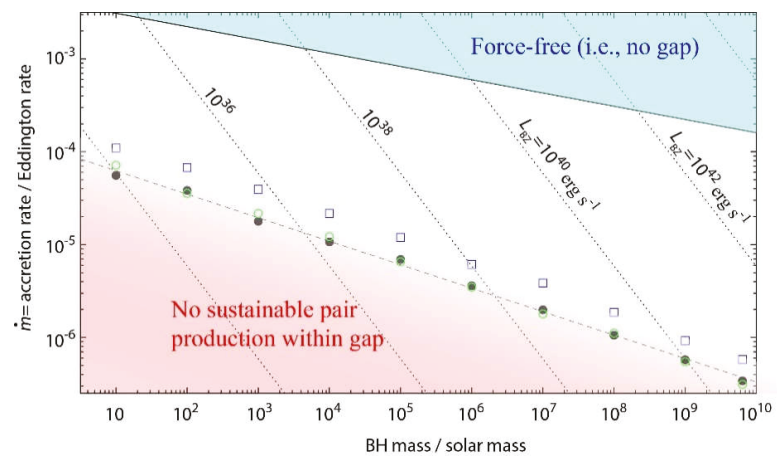

Figure 1. Gap formation region (white region) on the two-dimensional parameter space, $(M, \dot{m})$. The thick solid line shows the upper limit of the dimensionless accretion rate, $\dot{m}$, above which the copious pair production prevents the formation of a gap. The filled and open circles denote the lower limits of $\dot{m}$ (Section 5.4) for a stationary gap to exist in the case of $a / M=0.9$ and 0.5 , respectively. The thin dashed line shows a linear fit of the filled circles. The dotted lines show the Blandford-Znajek (BZ) power (Equation (4)) for an extremely rotating case, $a=M$. From [62].

Second, to examine the upper bound of the gap luminosity, we substitute $B=B_{\text {eq }}$ into Equation (4) to obtain the BZ power,

$$
L_{B Z}=1.7 \times 10^{46}\left(\frac{a}{M}\right)^{2} \dot{m} M_{9} \operatorname{erg~s}^{-1} .
$$

Putting $a=M$, we obtain the dotted lines in Figure 1 .

Third, substituting $\dot{m}=\dot{m}_{\text {up }}$ into Equation (9), we obtain the maximum gap luminosity (as the cross section of the solid and dotted lines),

$$
L_{B Z}=3.7 \times 10^{42}\left(\frac{a}{M}\right)^{2} M_{9}{ }^{6 / 7} \mathrm{erg} \mathrm{s}^{-1} .
$$

Assuming that $100 \%$ of this power is converted into radiation, we obtain the upper limit of the gap flux at Earth, $F_{\mathrm{BZ}}=L_{\mathrm{BZ}} / 4 \pi d^{2}$, where $d$ is the distance to the BH. Thus, for supermassive BHs, we obtain the following maximum flux,

$$
F_{B Z}=3.0 \times 10^{-10}\left(\frac{a}{M}\right)^{2} M_{9}^{6 / 7}\left(\frac{d}{10 \mathrm{Mpc}}\right)^{-2} \operatorname{ergs~s}^{-1} \mathrm{~cm}^{-2} .
$$

It follows that the IC component, which appears in VHE, may be detectable with ground-based IACTs. To quantify the actual flux as a function of the accretion rate, we must solve the gap electrodynamics. We will describe this issue in Sections 5-7.

\subsection{Criticism of the Stationary Black Hole (BH) Gap Model}

Levinson and Segev revisited the one-dimensional stationary BH gap model and found that the gap solutions are allowed only for small electric currents created within the gap [77]. They fully incorporated the GR effects in their basic equations, and solved the set of 1D Poisson equation, lepton Botzmann equations under mono-energetic approximation, and the radiative transfer equation, approximating all the IC photons gain the initial lepton kinetic energy, assuming a homogeneous and isotropic soft photon field (emitted by an ADAF). In their one-dimensional treatment, they found that $E_{\|}$is proportional to $w^{2}$, and the potential drop, $V_{\text {gap }}$, is proportional to $w^{3}$ (because the Poisson equation is a second-order differential equation), as Figure 2a indicates. They imposed a boundary condition such that the created charge density matches the local GJ charge density at the outer boundary; accordingly, $j_{\mathrm{cr}}$ is proportional $w$ (because of the Tayler expansion of $\rho_{\mathrm{GJ}}$ around the null 
surface). Thus, $E_{\|}$is proportional to $j_{\mathrm{cr}}{ }^{2}$, and $V_{\text {gap }}$ to $j_{\mathrm{cr}}{ }^{3}$. As a result of this strong dependence of $E_{\|}$ and $V_{\text {gap }}$ on $j_{\mathrm{cr}}$, they found that stationary solutions can be found only for smaller current densities $\left(j_{\text {LS }}<0.15\right.$ in their definition) (Figure $\left.2 b\right)$, where $j_{\text {LS }}$ denotes the dimensionless current introduced by Levinson and Segev,

$$
j_{\mathrm{LS}} \equiv \frac{J_{\mathrm{cr}} / 2}{\Omega_{\mathrm{F}} B_{\mathrm{H}} \cos \theta /(2 \pi)}=\frac{j_{\mathrm{cr}}}{2 \cos \theta}\left(\frac{r_{\mathrm{H}}}{r}\right)^{2}
$$

On the other hand, in the present review, we introduce the electric current per magnetic flux (Section 5.4),

$$
j_{\mathrm{cr}} \equiv \frac{J_{\mathrm{cr}}}{\omega_{H} B /(2 \pi)},
$$

where $J_{\mathrm{cr}}$ denotes the actual electric current. Thus, at $r \approx 1.4 r_{\mathrm{H}}$ and $\theta \approx 0{ }^{\circ}$ for $\Omega_{\mathrm{F}} \approx 0.5 \omega_{\mathrm{H}}, j_{\mathrm{LS}}=0.15$ corresponds to the conserved current of $j_{\mathrm{cr}} \approx 0.3$ in our present notation (Section 5.4). Note that $j_{\mathrm{cr}}$ is conserved along the individual magnetic flux tube.

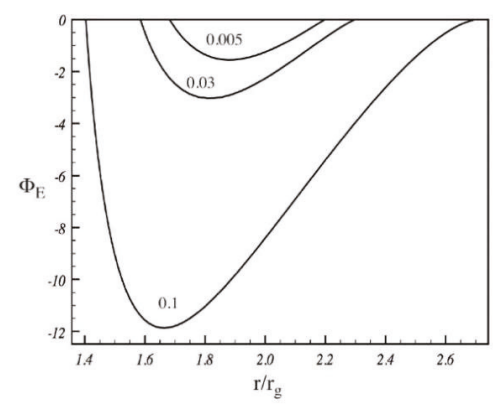

(a)

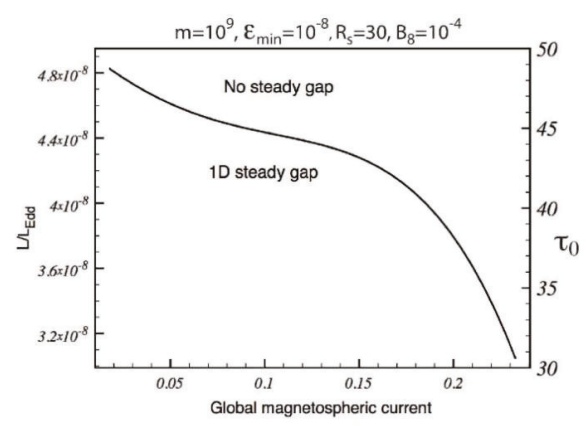

(b)

Figure 2. Stationary gap solutions for a billion solar-mass black hole (BH) for a split-monopole magnetic field line. (a) Renormalized acceleration electric field as a function of the Boyer-Lindquist radial coordinate. The three solid curves correspond to the solutions obtained for $j_{\mathrm{LS}}=0.005,0.03$, and 0.1 (Equation (12)). The peak of the non-corotational electric field, $\Phi_{\mathrm{E}}$, is proportional to the square of the created current. (b) Maximum Eddington rate (i.e., maximum gap luminosity normalized by the Eddington luminosity) that a stationary gap can attain for a fix magnetospheric current, $j_{\text {LS }}$ (abscissa). Adapted with permission from [77].

It is worth noting, however, that the 2D screening effect of $E_{\|}$becomes important when $w$ becomes comparable to or greater than the trans-field (e.g., meridional) thickness, $D_{\perp}$. In the case of pulsar OGs, $D_{\perp}$ becomes much less than the light cylinder radius, $c / \Omega_{\mathrm{F}}$, for young pulsars like the Crab pulsar, and comparable to $c / \Omega_{\mathrm{F}}$ for middle-aged pulsars like the Geminga pulsar (see $\S 2$ for references). For BHs, $D_{\perp} \sim r_{g}=M$ holds. Thus, in the present case, when $w \sim M, E_{\|}$saturates and suppressed 
when $j_{\text {cr }}>0.3$ (or $j_{\text {LS }}>0.15$ in the notation of [78]). In this section, we present the result of a pulsar case, whose electrodynamics is essentially the same as BHs. In Figure 3, we show the pulsar's $E_{\|}$ solved for three discrete gap currents, $j_{\mathrm{cr}}=0.01$ (dashed curve), 0.03 (solid curve), and 0.0592 (dotted curve). As the gap width approaches $D_{\perp}=3 \times 10^{8} \mathrm{~cm}, E_{\|}$is substantially suppressed by the 2D screening effect. As a result, pulsar OG solutions exist in a wide range of $j_{\mathrm{cr}}$, namely $0<j_{\mathrm{cr}}<1$. In the same way, for BHs, a stationary gap solution exists in a wide, $0<j_{\mathrm{cr}}<1$, by virtue of the $2 \mathrm{D}$ screening effect, as will be demonstrated in $§ 6.5$. In another word, the difficulty of stationary BH gap model pointed out by [77], can be solved if we consider the 2D electrodynamic structure.

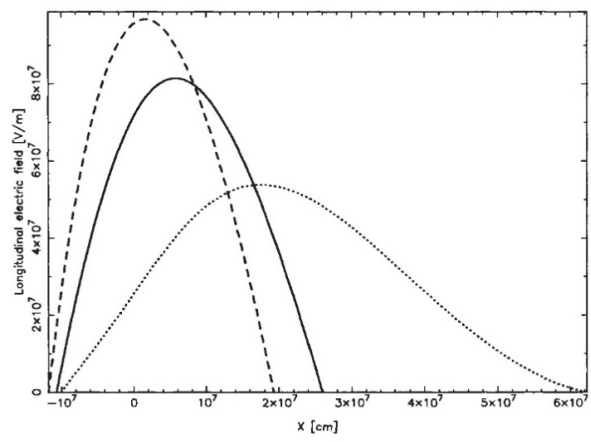

Figure 3. Acceleration electric field in a pulsar outer gap. The dashed, solid, and dotted curves correspond to the case of $j_{\mathrm{cr}}=0.01,0.03$, and 0.0592. From [78].

\subsection{Background Space-Time Geometry}

To examine the gap electrodynamics further quantitatively, let us describe the space time around a rotating $\mathrm{BH}$. Since the self-gravity of the accreting plasmas and the electromagnetic field is negligible compared to the $\mathrm{BH}^{\prime}$ s gravitational field, the spacetime around a rotating $\mathrm{BH}$ is well described by the Kerr metric [79]. In the Boyer-Lindquist coordinate [80], it becomes:

$$
d s^{2}=g_{t t} d t^{2}+2 g_{t \varphi} d t d \varphi+g_{r r} d r^{2}+g_{\theta \theta} d \theta^{2}+g_{\varphi \varphi} d \varphi^{2}
$$

where,

$$
g_{t t}=-\frac{\Delta-a^{2} \sin ^{2} \theta}{\Sigma}, g_{t \varphi}=-\frac{2 M a r \sin ^{2} \theta}{\Sigma}, g_{\varphi \varphi}=\frac{A \sin ^{2} \theta}{\Sigma}, g_{r r}=\frac{\Sigma}{\Delta}, g_{\theta \theta}=\Sigma,
$$

and,

$$
\Delta=r^{2}-2 M r+a^{2}, \Sigma=r^{2}+a^{2} \cos ^{2} \theta, A=\left(r^{2}+a^{2}\right)^{2}-\Delta a^{2} \sin ^{2} \theta .
$$

At the event horizon, $\Delta$ vanishes; thus, $r_{H}=r_{g}+\sqrt{r_{g}^{2}-a^{2}}$ gives the horizon radius. The Schwarzschild radius is given by $2 M$. If the $\mathrm{BH}$ is maximally rotating, $a \rightarrow M$ gives $r_{H} \rightarrow M$; that is, the horizon radius becomes half of the Schwarzschild radius. The space-time frame dragging angular frequency is given by:

$$
\omega \equiv-\frac{g_{t \varphi}}{g_{\varphi \varphi}}=\frac{2 M a r}{A} .
$$

which decreases as $\omega \rightarrow 2 \mathrm{Ma} / r^{3}$ away from the $\mathrm{BH}, r \gg M$. At the event horizon, $r=r_{\mathrm{H}}$, on the other hand, becomes the BH's spin angular frequency,

$$
\omega_{H} \equiv \frac{a}{2 M r_{H}} .
$$


which does not depend on $\theta$, as expected. To lower an index of a tensor, we can use the following relations,

$$
g^{t t}=-\frac{g_{\varphi \varphi}}{\rho_{w}{ }^{2}}, g^{\varphi \varphi}=-\frac{g_{t t}}{\rho_{w}{ }^{2}}, g^{t \varphi}=\frac{g_{t \varphi}}{\rho_{w}{ }^{2}}, g^{r r}=\frac{1}{g_{r r}}, g^{\theta \theta}=\frac{1}{g_{\theta \theta}},
$$

where $\rho_{w}{ }^{2} \equiv g_{t \varphi}{ }^{2}-g_{t t} g_{\varphi \varphi}=\Delta \sin ^{2} \theta$ vanishes at the horizon. We must choose appropriate vector and tensor components that are well-behaved at the horizon, as briefly discussed below in Equation (30).

\subsection{Non-Stationary BH Gap Model}

The aforementioned BH gap scenarios, however, are based on the stationary assumption. Thus, Levinson and Cerutti [81] examined the lepton acceleration and gamma-ray emission within a gap around a rotating $\mathrm{BH}$, performing one-dimensional particle-in-cell (PIC) simulations. From the homogeneous part of the Maxwell equations (i.e., the Faraday law), one obtains:

$$
\begin{aligned}
& \partial_{t} F_{r \theta}+\partial_{r} F_{\theta t}+\partial_{\theta} F_{t r}=0, \\
& \partial_{t} F_{\theta \varphi}+\partial_{\theta} F_{\varphi t}+\partial_{\varphi} F_{t \theta}=0, \\
& \partial_{t} F_{\varphi r}+\partial_{\varphi} F_{r t}+\partial_{r} F_{t \varphi}=0,
\end{aligned}
$$

where the electro-magnetic field strength tensor is related to the scalar and vector potentials by $F_{\mu v}=\partial_{\mu} A_{\nu}-\partial_{\nu} A_{\mu}$. They then investigated one-dimensional disturbances along the radial direction, setting $\partial_{\theta}=\partial_{\varphi}=0$. Thus, they obtained:

$$
\begin{gathered}
\partial_{t} F_{r \theta}=-\partial_{r} F_{\theta t}, \\
\partial_{t} F_{\theta \varphi}=0, \\
\partial_{t} F_{\varphi r}=-\partial_{r} F_{t \varphi},
\end{gathered}
$$

It follows from $\partial_{t} F_{\theta \varphi}=0$ that the radial component of the magnetic field should be time-independent. As an example, they assumed a split-monopole solution, fixing the magnetic flux function to be $A_{\varphi}(r, \theta)=C(1-\cos \theta)$, where $C$ is a constant. This solution can be obtained around a slowly rotating $\mathrm{BH}$ if we give the toroidal current by $J^{\varphi}=\mathrm{Cr}^{-4}$ on the equatorial plane $[13,26]$. With this solution of $A_{\varphi}$, the poloidal components of the magnetic field becomes time-independent and radial, because one obtains $F_{\theta \varphi}=\partial_{\theta} A_{\varphi}=C \sin \theta$ and $F_{\varphi r}=-\partial_{r} A_{\varphi}=0$. Accordingly, we obtain $F_{\varphi t}=\partial_{\varphi} A_{t}-\partial_{t} A_{\varphi}=0$; thus, the toroidal electric field vanishes. The poloidal components of the electric field are solved from the inhomogeneous part of the Maxwell equations (i.e., Amprere's law),

$$
\nabla_{\mu} F^{v \mu}=\frac{1}{\sqrt{-g}} \partial_{\mu}\left(\sqrt{-g} F^{v \mu}\right)=4 \pi J^{v},
$$

where $J^{v}$ refers to the electric four current. Putting $v=r$ and, and noting that

$$
\frac{\partial F^{r t}}{\partial t}=g^{r r}\left(g^{t t} \partial_{t} F_{r t}+g^{t \varphi} \partial_{t} F_{r \varphi}\right)=-\frac{A}{\Sigma^{2}}\left(\frac{\partial F_{r t}}{\partial t}+\omega \frac{\partial F_{r \varphi}}{\partial t}\right)
$$

and,

$$
\frac{\partial F^{\theta t}}{\partial t}=g^{\theta \theta}\left(g^{t t} \partial_{t} F_{\theta t}+g^{t \varphi} \partial_{t} F_{\theta \varphi}\right)=-\frac{A}{\Delta \Sigma^{2}}\left(\frac{\partial F_{\theta t}}{\partial t}+\omega \frac{\partial F_{\theta \varphi}}{\partial t}\right),
$$

holds, we obtain:

$$
\partial_{t} F_{r t}=\frac{\Sigma^{2}}{A} \partial_{\theta}(\ln \sqrt{-g}) F^{r \theta}-\frac{\Sigma^{2}}{A} \cdot 4 \pi J^{r}
$$


and,

$$
\partial_{t} F_{\theta t}=-\frac{\Delta \Sigma^{2}}{A} \partial_{r}(\ln \sqrt{-g}) F^{r \theta}-\frac{\Delta \Sigma^{2}}{A} \partial_{r} F^{r \theta}-\frac{\Delta \Sigma^{2}}{A} \cdot 4 \pi J^{\theta},
$$

where $F_{r \theta}=g_{r r} g_{\theta \theta} F^{r \theta}=\left(\Sigma^{2} / \Delta\right) F^{r \theta}$; also, $\partial_{\theta}=\partial_{\varphi}=0$ and $\partial_{t} F_{\theta \varphi}=\partial_{t} F_{\varphi r}=0$ are used. Thus, they solve the time evolution of $F^{r \theta}, F_{r t}$, and $F_{\theta t}$, all of which are well-behaved at the horizon, from Equations (24), (29) and (30). The electric current density $J^{v}$ in Ampere's law is constructed from the actual motion of the charged particles in the PIC scheme.

By this one-dimensional, general-relativistic (GR) PIC simulation scheme, they demonstrated that $E_{\|}$is efficiently screened out by the discharge of created pairs within the duration that is comparable to the dynamical time scale, $r_{\mathrm{g}} / c=G M / c^{3}=M$, that the residual $E_{\|}$leads to a strong flare of VHE curvature radiation, which is followed by a state of self-sustained, rapid plasma oscillations, and that the resultant quasi-stationary gamma-ray luminosity attains only about $10^{-5}$ of the BZ power; that is, the time-averaged luminosity becomes less than the stationary analysis. See also Levinson et al., who semi-analytically found longitudinal oscillations in their two beam model [82].

Figure 4 exhibits the snapshots at four discrete simulation times. The upper panel shows the variation of the pair and photon densities. Pair density increases due to pair production from zero and saturates at about 10 times GJ value within $t=5 \mathrm{GM} / \mathrm{c}^{3}=5 \mathrm{M}$, that is, within a few dynamical time scales, where we may define the dynamical time scale to be $2 \mathrm{GM} / \mathrm{c}^{3}=2 \mathrm{M}$. Accordingly, $E_{\|}$is nearly screened out. The sporadic pair creation leads to rapid spatial and temporal oscillations of $E_{\|}$(middle panel) and $J^{r}$ (bottom panel). Above nearly 10 dynamical time scales, $t>20 M$, pair and photon distribution function attain quasi-stationary state.
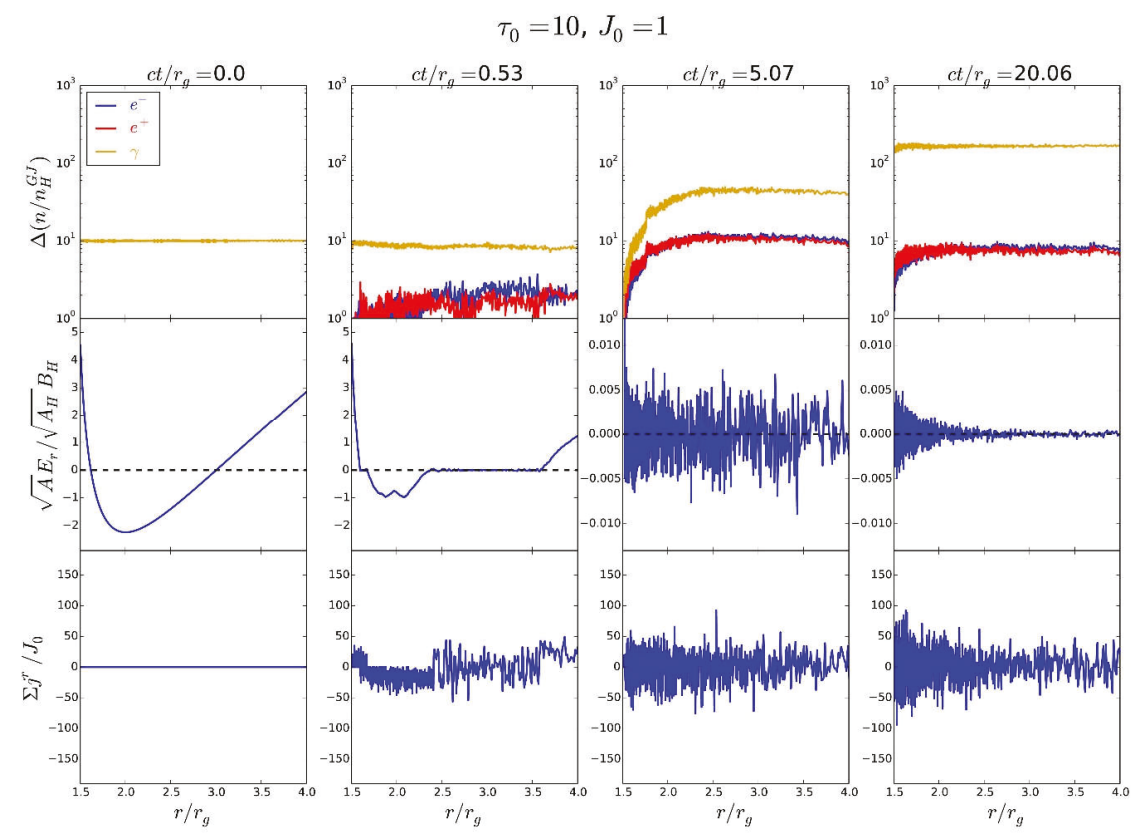

Figure 4. Snapshots from a typical simulation of spark gap dynamics. Shown are the pair and photon densities (upper panels), electric flux (middle panels), and radial electric current, $\Sigma J^{r}$, normalized by the global magnetospheric current $J_{0}$. The leftmost panels delineate the initial state, at $t=0$. The rightmost panels show the relaxed state, following the prompt discharge. We note the scale change on the vertical axis in the middle panels. Adapted with permission from [81]. 
Figure 5 exhibits the light curve evolution. It follows that the gamma-ray luminosity approaches the $\mathrm{BZ}$ power during the initial spike but decays to the terminal value as $E_{\|}$is screened out. It is possible to argue that strong, rapid flares should be produced every time a magnetospheric gap is restored. The flaring episode is then followed by quiescent emission with a luminosity, $L_{\gamma} \sim 10^{-5} L_{\mathrm{BZ}}$.

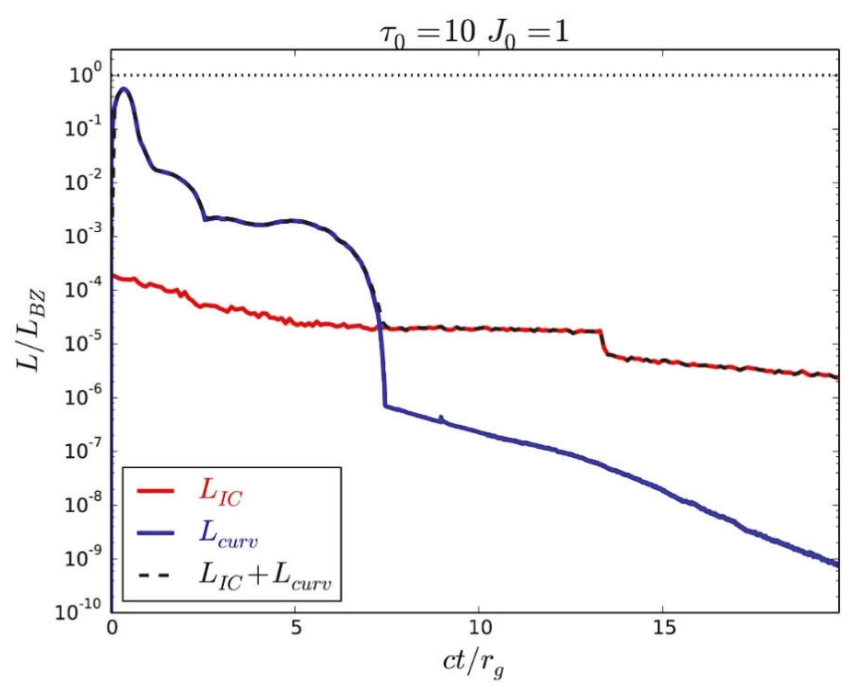

Figure 5. Gamma-ray light curve produced by the gap discharge. The red line corresponds to inverse-Compton (IC) emission, the blue line to curvature emission, and the black dashed line to the sum of both components. Adapted with permission from [81].

More recently, Chen et al. performed 1D PIC simulations on the BH gap model [83]. They assumed a Minkowski spacetime in the Maxwell equations, lepton equation of motion, and radiative transfer equation, except for the GJ charge density, whose GR expression is essential for the formation of a null surface near the event horizon, whereas full GR expressions were implemented in all the basic equations in [81]. Cheng et al. adopted a Newtorian split-monopole solution [84], and solved the time evolution of $E_{\|}=E_{r}, E_{\theta}$, and $B_{\varphi}$. It is found that a $\mathrm{BH}$ gap opens quasi-periodically and the screening of $E_{\|}$creates oscillations. Eventually, when the created pairs escape across the light surfaces, the charge-starved magnetosphere recur in the formation of a gap.

Figure 6 shows the third cycle of such a gap development. The middle panel in the top indicates that $E_{\|}$arises around the null surface, which is shown in the third panels as the crossing of the GR charge density (green curve in the orange band) and 0 (horizontal green line). However, the gap can also appear at different positions, as the right two panels in the top row show. The gap (shown in the top row) appears in the low multiplicity region, as indicated in the second row. Note that the green curve in the bottom panels show the spectra of the photons that materialize within the simulation box; thus, they do not show the gamma-ray spectra to be observed.

In short, time-dependent, one-dimensional GR PIC simulations revealed that gaps are unsteady but tend to a quasi-steady state or a quasi-periodical state. The former, quasi-steady solution shows that the time-averaged gap gamma-ray luminosity is of an order of magnitude smaller than stationary analysis because of the efficient screening [81]. Nevertheless, in the present review we focus on the stationary $\mathrm{BH}$ gap scenario in order to elucidate the detailed gap electrodynamics, which will be also important to understand the time-dependent phenomena. 


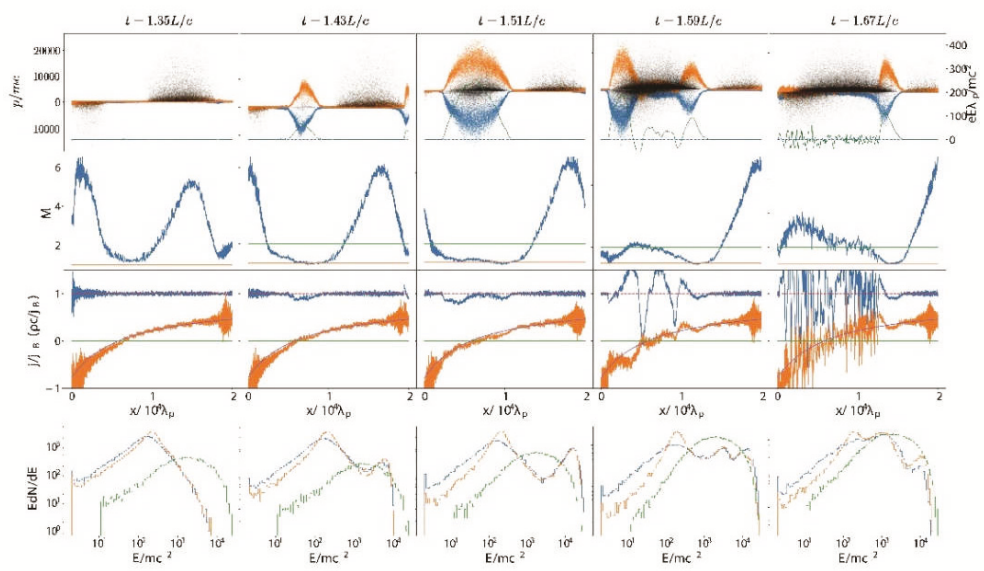

Figure 6. Time evolution of the gap. From left to right are snapshots at labeled times, where $\mathrm{L}$ is the size of the box. The panels from top to bottom are: (1) phase space plots for electrons (blue), positrons (orange), and photons (black). The green line is electric field and its scale is on the right. (2) Pair multiplicity $M$. Orange and green lines mark $M=1$ and 2, respectively. (3) Current j (blue) and charge density (orange) and their background values. (4) Spectrum of electrons (blue), positrons (orange), and photons (green). Adapted with permission from [83].

\section{Advection-Dominated Accretion Flow}

Before moving onto the gap electrodynamics, let us briefly describe the radiatively inefficient accretion flow in the lower latitudes (i.e., near the equator), because it is essential to quantify the photon-photon pair production and IC scatterings taking place inside and outside the gap in the higher latitudes (i.e., in the polar funnel). Since we are concerned with the soft photon field near the $\mathrm{BH}$, we do not consider the structure of accretion flows away from the $\mathrm{BH}$, focusing on an ADAF, a representative model of radiatively inefficient accretion flow. We consider that plasma accretion takes place near the rotational equator with a certain vertical thickness (e.g., $[20,85,86])$. Such an accretion cannot penetrate into the higher latitudes, that is, in the polar funnel because the centrifugal-force barrier prevents plasma accretion toward the rotation axis, and because the timescale for magnetic Rayleigh-Taylor instability or turbulent diffusion is long compared to the dynamical timescale of accretion. In this evacuated funnel, the poloidal magnetic field lines resemble a split monopole in a time-averaged sense [22]. The lower-latitude accretion emits $\mathrm{MeV}$ photons into the higher latitudes, supplying electron-positron pairs in the funnel. If the pair density in the funnel becomes less than $N_{\mathrm{GJ}}$, $E_{\|}$arises. Thus, a plasma accretion in the lower altitudes and a gap formation in the higher altitudes are compatible in a $\mathrm{BH}$ magnetosphere.

Since the gap luminosity increases with decreasing accretion rate $[61-63,67,68]$, we consider a dimensionless accretion rate that is much small compared to unity. Such a low accretion status corresponds to the quiescence or low-hard state for X-ray binaries, and low luminosity active galactic nuclei for super-massive BHs. At such a low accretion rate, the equatorial accretion flow becomes optically thin for Bremsstrahlung absorption and radiatively inefficient because of the weak Coulomb interaction between the ions and electrons. Accordingly, the ions store the heat within the flow itself and accretes onto the $\mathrm{BH}$ without losing the thermal energy as radiation. This radiatively inefficient flow can be described by an ADAF [28,29,87-92], and provides the target soft photons for the IC-scattering and the photon-absorption processes in the polar funnel. Thus, to tabulate the redistribution functions for these two processes, we compute the specific intensity of the ADAF-emitted photons. For this purpose, we adopt the analytical self-similar ADAF spectrum presented in [90]. The spectrum includes the contribution of the synchrotron, IC, and Bremsstrahlung processes. These three cooling mechanisms 
balance with the heating due to the viscosity and the energy transport form ions, and determine the temperature of the electrons in an ADAF to be around $T_{\mathrm{e}} \sim 10^{9} \mathrm{~K}$. In radio wavelength, the ADAF radiation field is dominated by the synchrotron component whose peak frequency, $v_{\mathrm{c}, \text { syn, }}$, varies with the accretion rate as $v_{\mathrm{c}, \text { syn }} \propto \dot{m}^{1 / 2}$. In the X-ray wavelength, the Bremsstrahlung component dominates the ADAF flux at such a low $\dot{m}$. In soft gamma-ray wavelength, this component cuts off around the energy $h v \sim k T_{e}$. These MeV photons (with energies slightly below $k T_{e}$ collide each other to materialize as seed electrons and positrons that initiate a pair-production cascade within the gap. If the accretion rate is as low as $\dot{m}<\dot{m}_{\text {up }}(M, a)<10^{-2.5}$, the seed pair density becomes less than the GJ value [59], thereby leading to an occurrence of a vacuum gap in the funnel.

It is noteworthy, however, that at low accretion rate, namely $\dot{m}<10^{-3}$, less efficient thermalization may lead to a hybrid thermal-nonthermal energy distribution. Observationally, it is indeed known that non-thermal electrons are needed to reproduce the quiescent low-frequency radio emission in Sgr A* [93-95] and other low-luminosity AGNs [96]. Theoretically, the direct heating of electrons (i.e., not via Coulomb collisions with ions) is proposed via several mechanisms, such as magnetic reconnection [97-101], MHD turbulence [98,102-105], or dissipation of pressure anisotropy in a collisionless plasma [106]. At the present stage, there is no consensus on the theory of electron heating yet. In the present review, we neglect such direct heating of electrons and assume that only less than $1 \%$ of the ions thermal energy is transferred to electrons. However, if more gravitational energy is converted into electrons' heat by direct heating (i.e., if the fraction of electron heating becomes " 0.01 ), the increased ADAF soft photon field would significantly reduce the gap luminosity at a fixed accretion rate. We should keep this point in mind when we construct a BH gap model, irrespective of whether the gap is stationary or non-stationary. We will present the input ADAF spectrum when we show the predicted gamma-ray spectrum of the gap emissions.

\section{Stationary, Two-Dimensional Gap Electrodynamics}

Next, we formulate the BH gap, which will be formed when $\dot{m}<\dot{m}_{\text {up }}$.

\subsection{Poisson Equation for the Non-Corotational Potential}

In a stationary and axisymmetric spacetime, Gauss's law for the electrostatic potential gives

$$
\nabla_{\mu} F^{t \mu}=\frac{1}{\sqrt{-g}} \partial_{\mu}\left[\frac{\sqrt{-g}}{\rho_{w}^{2}} g^{\mu v}\left(-g_{\varphi \varphi} F_{t v}+g_{t \varphi} F_{\varphi v}\right)\right]=4 \pi \rho,
$$

where $\nabla$ denotes the covariant derivative, $\sqrt{-g}=\sqrt{g_{r r} g_{\theta \theta} \rho_{w}{ }^{2}}=\Sigma \sin \theta, \rho_{w}{ }^{2}=\Delta \sin ^{2} \theta$, and denotes the real charge density; the Greek indices run over $t, r, \theta, \varphi$. The electro-magnetic field strength tensor $F_{\mu \nu}$ is defined in Section 3.6.

In the present formalism, we assume that the electromagnetic fields depend on $t$ and $\varphi$ only through $\varphi-\Omega_{F} t$, where $\Omega_{F}$ denotes the angular frequency of the magnetic field rotation. Imposing the ideal MHD condition (i.e., imposing a very large magnetic Reynolds number in the Ohm's law, which leads to a vanishing electric field in the co-moving frame of the fluid), we obtain $F_{r t}=\Omega_{F} F_{\varphi r}$ and $F_{\theta t}=-\Omega_{F} F_{\theta \varphi}$. Under this assumption of stationarity, $F_{\mu v}=F_{\mu v}\left(r, \theta, \varphi-\Omega_{F} t\right)$, we can introduce the non-corotational potential such that:

$$
F_{\mu t}+\Omega_{F} F_{\mu \varphi}=\partial_{\mu} \Phi\left(r, \theta, \varphi-\Omega_{F} t\right),
$$

If $F_{A t}+\Omega_{F} F_{A \varphi}$ vanishes for $A=r$ and $\theta, \Omega_{F}$ is conserved along the magnetic field line. However, in a lepton accelerator, $F_{A t}+\Omega_{F} F_{A \varphi}$ deviates from 0 and the magnetic field lines do not rigidly rotate. The deviation from rigid rotation is expressed by how $\Phi$ deviates from a constant value at each place in the magnetosphere. 
We adopt the static observer whose four velocity is given by the Killing vector, $\xi^{\mu}=(1,0,0,0)$. Then the electromagnetic fields have the following components in the Boyer-Lindquist coordinates, $E_{r}=F_{r t}, E_{\theta}=F_{\theta t}, E_{\varphi}=F_{\varphi t}$, and $[107,108]$

$$
B^{r}=-\frac{g_{t t}+g_{t \varphi} \Omega_{F}}{\sqrt{-g}} F_{\theta \varphi}, B^{\theta}=-\frac{g_{t t}+g_{t \varphi} \Omega_{F}}{\sqrt{-g}} F_{\varphi r}, B_{\varphi}=\sqrt{-g} F^{r \theta} .
$$

The acceleration electric field is given by $E_{\|}=\mathbf{E} \cdot \mathbf{B} / B=\left(F_{i t}+\Omega_{F} F_{i \varphi}\right) B^{i} / B=(\mathbf{B} / B) \cdot(\nabla \Phi)$, where $B \equiv|\mathbf{B}|$. Note that $\left(E_{\|} B\right)^{2}=(\mathbf{E} \cdot \mathbf{B})^{2}=\operatorname{det}(F)$ is a frame-independent scalar.

Substituting Equation (30) into (29), we obtain the Poisson equation for the non-corotational potential,

$$
\frac{1}{\sqrt{-g}} \partial_{\mu}\left[\frac{\sqrt{-g}}{\rho_{w}^{2}} g^{\mu v} g_{\varphi \varphi} \partial_{v} \Phi\right]=4 \pi\left(\rho-\rho_{\mathrm{GJ}}\right),
$$

where the general-relativistic Goldreich-Julian (GJ) charge density is defined by

$$
\rho_{\mathrm{GJ}} \equiv \frac{1}{4 \pi \sqrt{-g}} \partial_{\mu}\left[\frac{\sqrt{-g}}{\rho_{w}^{2}} g^{\mu v} g_{\varphi \varphi}\left(\Omega_{F}-\omega\right) F_{\varphi v}\right],
$$

where $\omega \equiv-g_{t \varphi} / g_{\varphi \varphi}$ denotes the frame dragging angular frequency. Away from the horizon, $r \gg M$, Equation (35) reduces to the standard, special-relativistic expression of the GJ charge density,

$$
\rho_{\mathrm{GJ}}=-\frac{\Omega \cdot \mathbf{B}}{2 \pi c}+\frac{(\Omega \times \mathbf{r}) \cdot(\nabla \times \mathbf{B})}{4 \pi c}
$$

Not only $F_{\mu \nu}$ and $\Phi$ but also $\rho$ may depend on $t$ and $\varphi$ through $\varphi-\Omega_{F} t$. In this case, Equation (34) gives "stationary" gap solution in the "co-rotational" frame, in the sense that $\Phi$ and $\rho$ are a function of $r, \theta$, and $\varphi-\Omega_{F} t$. Note that such stationary solutions are valid not only between the two light surfaces where $k_{0} \equiv-g_{t t}-2 g_{t \varphi} \Omega_{F}-g_{\varphi \varphi} \Omega_{F}^{2}>0$, but also inside the inner light surface and outside the outer light surface where $k_{0}<0$ [109-111].

\subsection{The Null-Charge Surface}

Equation (34) shows that $E_{\|}$is exerted along $\boldsymbol{B}$ if $\rho$ deviates from $\rho_{\mathrm{GJ}}$. In a stationary gap, the derivative of $E_{\|}$along $B$ should change sign at the outer and the inner boundaries, where the outer boundary is located at larger distance from the hole, while the inner boundary is located near the hole. Thus, a vacuum gap (with $\rho \approx 0$ ) is located around the null-charge surface, on which $\rho_{\mathrm{GJ}}$ changes sign. If the gap becomes non-vacuum (i.e., $|\rho|$ is slight smaller than $\left|\rho_{\mathrm{GJ}}\right|$ ), the gap position is essentially unchanged (6.6). Thus, the null-charge surface becomes the natural place for a gap to arise, in the same way as the pulsar outer-gap model (for an analytic argument of the gap position, see 2 of [112]).

In the case of $\mathrm{BHs}$, the null-charge surface appears near the surface on which $\omega$ coincides with $\Omega_{F}$ [52]. Since $\omega$ matches $\Omega_{F}$ only near the horizon, the gap inevitably appears near the horizon, irrespective of the $\mathrm{BH}$ mass. In Figure 7, we plot the distribution of the null surface as the thick red solid curve. In the left panel, we assume that the magnetic field is split-monopole, adopting $A_{\varphi}=C(1-\cos \theta)$ as the magnetic flux function [13], where $C$ is a constant. In the right panel, on the other hand, we assume a parabolic magnetic field line with $A_{\varphi}=(C / 2) r(1-\cos \theta)$ on the poloidal plane (i.e., $r-\theta$ plane). We adopt $\Omega_{F}=0.3 \omega_{H}$ for both cases $[20,113]$. It follows that the null surface distributes nearly spherically, irrespective of the poloidal magnetic field configuration. This is because its position is essentially determined by the condition $\omega(r, \theta)=\Omega_{F}\left(A_{\varphi}\right)$, because $\omega$ has weak dependence on $\theta$, and because we assume $\Omega_{F}$ is constant for $A_{\varphi}$ for simplicity. If $\Omega_{F}$ decreases (or increases) toward the polar region, the null surface shape becomes prolate (or oblate). The field angular frequency $\Omega_{F}$ is, indeed, deeply related to the accretion conditions. For instance, in order to get adequate jet efficiency from the magnetic field in the funnel above the $\mathrm{BH}$ for a radio-loud active 
galactic nuclei (AGNs), one needs significant flux compression from the lateral boundary imposed by the accretion flow and corona [114]. Although it is not a self-consistent solution, we adopt a constant $\Omega_{F}$ in the present review for simplicity.

Let us quickly take a look at the case of slower and faster rotations. Figure 8 shows the GJ charge density and the null-charge surface distribution when the poloidal magnetic field line is radial (i.e., split-monopole); the $\mathrm{BH}$ mass and spin are the same as Figure 7 . The left panel corresponds to the case of $\Omega_{F}=0.15 \omega_{H}$, while the right panel $\Omega_{F}=0.60 \omega_{H}$. It follows that the null-charge surface approaches the horizon for as $\Omega_{F}$ increases. In what follows, we adopt $\Omega_{F}=0.5 \omega_{H}$ unless explicitly mentioned, because the BZ power maximizes at this magnetic-field rotation.

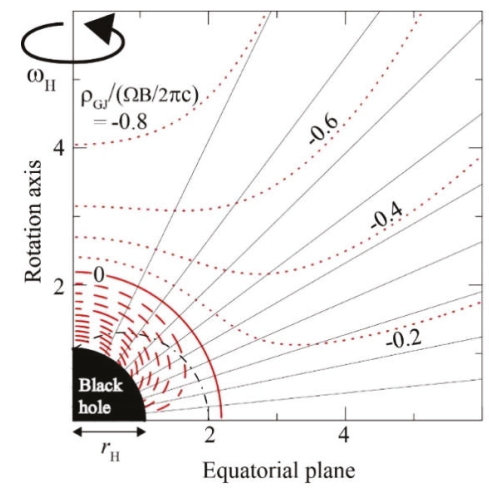

(a)

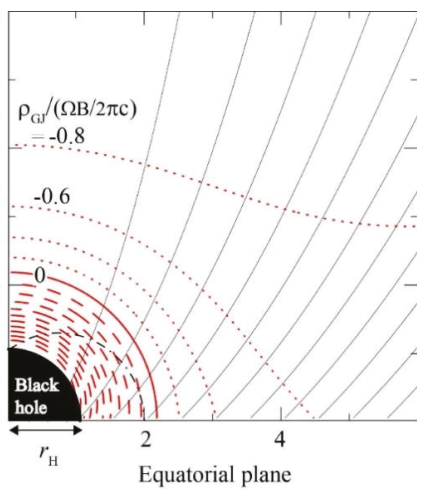

(b)

Figure 7. Distribution of the null surface (red thick solid curve) on the poloidal plane in the Boyer-Lindquist coordinates. The axes are in $r_{\mathrm{g}}=G M / c^{2}=M$ unit. The black hole (filled black region in the lower left corner) rotates rapidly with spin parameter $a=0.998 \mathrm{M}$ around the ordinate. The contours of the dimensionless Goldreich-Julian charge density are plotted with the red dashed curves (for positive values) and the red dotted ones (for negative values as labeled). The black dash-dotted curve denotes the static limit, within which the rotational energy of the hole is stored. The black solid curves denote the magnetic field lines. (a) The case of a split-monopole magnetic field. (b) The case of a parabolic field. From [61].

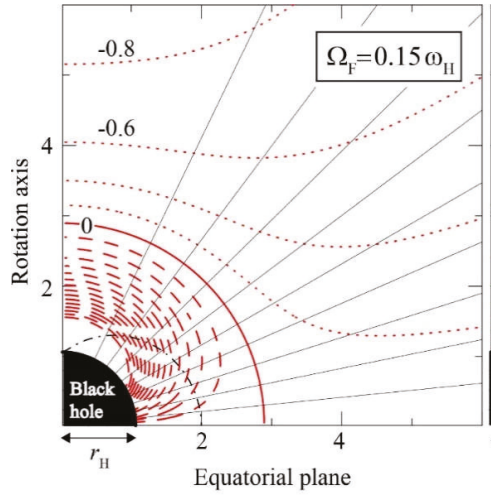

(a)

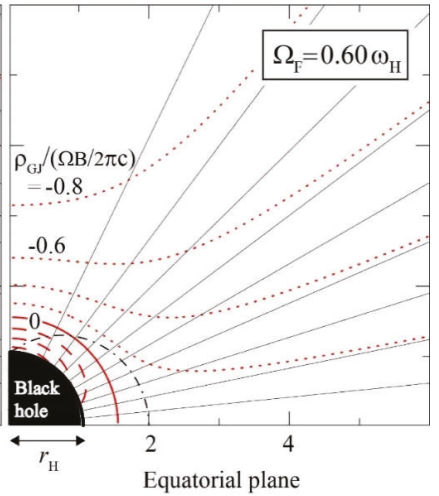

(b)

Figure 8. Similar to the left panel of Figure 7: both panels show the dimensionless Goldreich-Julian charge density (red dashed, solid, and dotted contours) for a split-monopole magnetic field (black solid lines). (a) The case of a slower field-line rotation, $\Omega_{F}=0.15 \omega_{H}$. (b) The case of a faster rotation, $\Omega_{F}=0.60 \omega_{H}$. From [61]. 


\subsection{The Stagnation Surface}

In a stationary and axisymmetric $\mathrm{BH}$ magnetosphere, an MHD fluid flows from a greater $k_{0}$ region to a smaller $k_{0}$ one. It follows that both the inflows and outflows start from the two-dimensional surface on which $k_{0}$ maximizes along the poloidal magnetic field line. Thus, putting $k_{0}{ }^{\prime}=0$, we obtain the position of the stagnation surface (thick green solid curve in Figure 9), where the prime denotes the derivative along the poloidal magnetic field line. The condition $k_{0}{ }^{\prime}=0$ is equivalent to imposing a balance among the gravitational, centrifugal, and Lorentz forces on the poloidal plane. In Figure 9, we plot the contours of $k_{0}$ for split-monopole and parabolic poloidal magnetic field lines.

It is clear that the stagnation surface is located at $r<5 \mathrm{M}$ in the lower latitudes but at $r>5 \mathrm{M}$ in the higher latitudes. As the poloidal field geometry changes from radial to parabolic, the stagnation surface moderately moves away from the rotation axis in the higher latitudes.

This analytical argument of the position of the stagnation surface [115], was confirmed by general relativistic (GR) MHD simulations [60]. In these numerical works, the stagnation surface is time-dependent but stably located at 5-10 M with a prolate shape, as depicted in Figure 9.

The two-dimensional surfaces on which $k_{0}$ vanishes, are called the outer and inner light surfaces (thick red solid curves in Figure 9). Outside the outer light surface (or inside the inner light surface), plasma particles must flow outwards (or inwards).

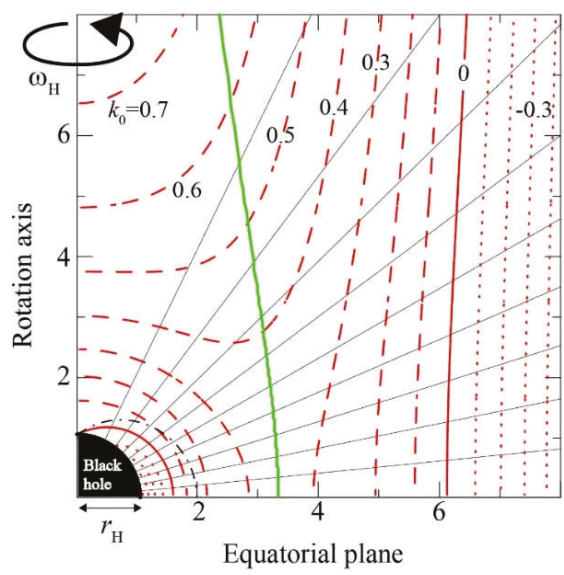

(a)

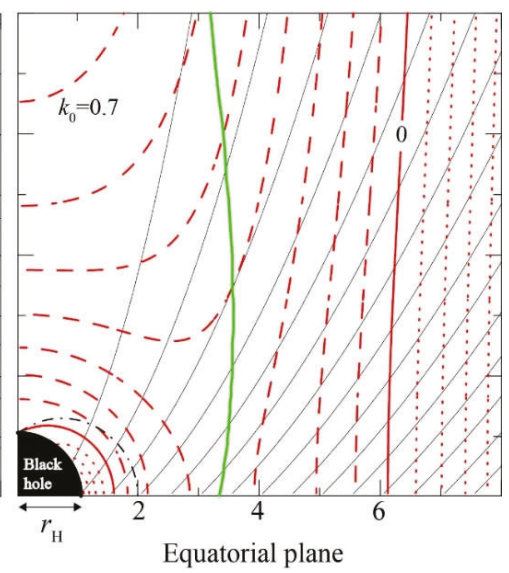

(b)

Figure 9. Distribution of the stagnation surface (thick green solid curve) on the poloidal plane. Similar to Figure 7 , but equi- $k_{0}$ potential curves are depicted with the dashed curves (for positive values) and dotted ones (for negative values). The thick, red solid curves denote the outer and inner light surfaces where $k_{0}$ vanishes. (a) The case of a split-monopole magnetic field. (b) The case of a parabolic field. From [61].

\subsection{The Gap Position}

To examine the Poisson Equation (34), it is worth noting that the distribution of $\rho_{\mathrm{GJ}}$ has relatively small dependence on the magnetic field geometry near the horizon, because its distribution is essentially governed by the space-time dragging effect, as indicated by the red solid, dashed, and dotted contours in Figure 7 for split-monopole and parabolic fields. As a result, the gap solution little depends on the magnetic field line configuration, particularly in the higher latitudes. In what follows, we thus assume a split-monopole field and adopt a constant $\Omega_{F}$ for $A_{\varphi}$ for simplicity, as described in the left panels of Figures 7 and 9.

In a pulsar magnetosphere, the gap position is located around the null-charge surface if there is no leptonic injection across either of the inner and outer boundaries, and that the gap position 
shifts outwards (or inwards) when leptons are injected into the gap across the inner (or the outer) boundary $[116,117]$. Since the gap electrodynamics is essentially the same between pulsar outer gaps and $\mathrm{BH}$ gaps, we can expect that the same conclusion holds in the case of $\mathrm{BH}$ magnetospheres.

In a BH magnetosphere, a gap indeed distributes around the null-charge surface, as will be shown in Sections 6.3 and 7.2 .

\subsection{Advection-Dominated Accretion Flow (ADAF)-Emitted Soft Photon Field in the Magnetosphere}

To quantify the gap electrodynamics, we need to compute the pair creation rate. To this end, we must tabulate the specific intensity of the soft photons at each position in the polar funnel. In this section, we therefore consider how the soft photons are emitted in an ADAF and propagate around a rotating $\mathrm{BH}$. We assume that the soft-photon field is axisymmetric with respect to the $\mathrm{BH}$ rotation axis. When the mass accretion rate is much smaller than the Eddington rate, the accreting plasmas form an ADAF with a certain thickness in the equatorial region. For simplicity, in this paper we approximate that such plasmas rotate around the BH with the GR Keplerian angular velocity,

$$
\Omega_{K} \equiv \pm \frac{1}{M} \frac{1}{(r / M)^{3 / 2} \pm a / M}
$$

and that their motion is dominated by this rotation. That is, we neglect the motion of the soft-photon-emitting plasmas on the poloidal plane, $(r, \theta)$, for simplicity.

Let us introduce the local rest frame (LRF) of such rotating plasmas. The orthonormal tetrad of the LRF is given by [63],

$$
\begin{gathered}
\mathbf{e}_{(\hat{t})}{ }^{\mathrm{LRF}}=\left(\frac{d t}{d \tau}\right)^{\mathrm{LRF}}\left(\partial_{t}+\Omega_{K} \partial_{\varphi}\right), \\
\mathbf{e}_{(\hat{\varphi})}^{\mathrm{LRF}}=\frac{g_{t \varphi}+g_{\varphi \varphi} \Omega_{K}}{\rho_{w} \sqrt{D}} \partial_{t}-\frac{g_{t t}+g_{t \varphi} \Omega_{K}}{\rho_{w} \sqrt{D}} \partial_{\varphi}, \\
\mathbf{e}_{(\hat{r})}^{\mathrm{LRF}}=\sqrt{g^{r r}} \partial_{r}, \mathbf{e}_{(\hat{\theta})}^{\mathrm{LRF}}=\sqrt{g^{\theta \theta}} \partial_{\theta},
\end{gathered}
$$

where,

$$
D \equiv-g_{t t}-2 g_{t \varphi} \Omega_{K}-g_{\varphi \varphi} \Omega_{K}{ }^{2},
$$

and the redshift factor between the LRF and the distant static observer becomes:

$$
\left(\frac{d \tau}{d t}\right)^{\mathrm{LRF}}=\sqrt{\mathrm{D}}
$$

Note that the GR Keplerian angular frequency on the equator, $\Omega_{K}$, is different from the magnetic-field angular frequency, $\Omega_{\mathrm{F}}$. Thus, $D$ is not $k_{0}$. Note also that Equation (42) is the reciprocal of what appears in Equation (38). For further details on how to compute the specific intensity of the ADAF soft photon field, see [63].

Between the distant static observer (i.e., us) and the LRF, the photon energy changes by the redshift factor,

$$
\frac{\omega_{\infty}}{\omega_{\mathrm{LRF}}}=\frac{\mathbf{e}_{(t)}^{\infty} \cdot \mathbf{k}}{\mathbf{e}_{(t)}{ }_{\mathrm{LRF}} \cdot \mathbf{k}}=\left(\frac{d t}{d \tau}\right)^{\mathrm{LRF}} \frac{-\hbar \omega_{\infty}}{\left(\mathbf{e}_{(t)}+\beta^{\varphi} \mathbf{e}_{(\varphi)}\right) \cdot \mathbf{k}}=\left(\frac{d t}{d \tau}\right)^{\mathrm{LRF}} \frac{1}{1-\beta^{\varphi} \lambda},
$$

where $\lambda \equiv k_{\varphi} / \hbar \omega_{\infty}$ denotes the ratio between the photon angular momentum $k_{\varphi}$ and energy $-k_{t}=$ $\hbar \omega_{\infty}$, both of which are conserved along the ray. 
We tabulate the soft photon specific intensity at each position in the magnetosphere by the ray-tracing method. The dispersion relation $k^{\mu} k_{\mu}=0$ gives the Hamiltonian,

$$
H=-k_{t}=-\frac{g_{t \varphi}}{g_{\varphi \varphi}} k_{\varphi} \pm \frac{\rho_{w}}{g_{\varphi \varphi}} \sqrt{k_{\varphi}^{2}+g_{\varphi \varphi}\left(g^{r r} k_{r}^{2}+g^{\theta \theta} k_{\theta}^{2}\right)},
$$

Thus, the Hamilton-Jacobi relation gives $d r / d t, d \theta / d t, d k_{r} / d t$, and $d k_{\theta} / d t$ in terms of $(r, \theta)$ and $\left(k_{r}, k_{\theta}\right)$ for a fixed $\left(-k_{t}, k_{\varphi}\right)$, which enables us to ray-trace the soft photons in the Kerr space time [63].

In what follows, we compute the collision frequencies of two-photon pair creation and IC scatterings in the frame of a zero-angular-momentum observer (ZAMO), which rotates with the same angular frequency as the space-time dragging frequency, $\omega=-g_{t \varphi} / g_{\varphi \varphi}$. Putting $\beta^{\varphi}=\omega$, we obtain the lapse (Section 3.3 of [63]):

$$
\frac{d \tau}{d t}=\alpha=\frac{\rho_{w}}{\sqrt{g_{\varphi \varphi}}}=\sqrt{\frac{\Delta \Sigma}{A}}
$$

Between the distant static observer and ZAMO, the photon energy changes by the redshift factor,

$$
\frac{\omega_{\infty}}{\omega_{\mathrm{ZAMO}}}=\frac{\mathbf{e}_{(t)}{ }^{\infty} \cdot \mathbf{k}}{\mathbf{e}_{(t)} \mathrm{ZAMO}^{\mathrm{ZA}} \mathbf{k}}=\frac{\alpha}{1-\beta^{\varphi} \lambda},
$$

Combining Equations (46) and (58), we find that the photon energy changes from the LRF (in the disk) to ZAMO (in the magnetosphere including the polar funnel) by the factor:

$$
g_{\mathrm{s}} \equiv \frac{\omega_{\mathrm{ZAMO}}}{\omega_{\mathrm{LRF}}}=\alpha^{-1}\left(\frac{d \tau}{d t}\right)^{\mathrm{LRF}} \frac{1-\omega \lambda}{1-\Omega_{K} \lambda}
$$

By the ray-tracing technique described above (see also [118]), we can now compute the specific intensity of the soft photons emitted from the ADAF at each place in the magnetosphere in the ZAMO frame of reference. Integrating this ZAMO-measured specific intensity over the propagation solid angle in each directional bin at each point in ZAMO, we obtain the soft photon flux at each point in each direction. Finally, we use this flux to compute the IC optical depth and the photon-photon collision optical depth in ZAMO.

Let us define $\left(d F_{\mathrm{S}} / d E_{\mathrm{s}}\right)_{0}$ to be the differential soft photon flux that would be obtained if the specific intensity were homogeneous and isotropic in a Minkowski space time within the radius $6 M$, and if the total soft photon luminosity were given by radial flux at $6 M$ multiplied by $4 \pi(6 M)^{2}$. Then, the photon differential number flux at each place can be expressed as $d F_{\mathrm{S}} / d E_{\mathrm{S}}=f_{A D A F}(r, \theta)\left(d F_{\mathrm{S}} / d E_{\mathrm{S}}\right)_{0}$, where the flux correction factor can be computed by summing up the ray-traced photons at each place by the method described above. If the photon specific intensity were homogeneous and isotropic at $r<6 M$ in a flat spacetime, we would have $f_{A D A F}(r, \theta)=1$ at any $r$ and $\theta$ within the radius $6 M$.

Figure 10 shows the flux correction factor, $f_{A D A F}(r, \theta)$. The top left, top right, bottom left and bottom right panels show the results for colatitudes $\theta=0^{\circ}, 15^{\circ}, 30^{\circ}, 45^{\circ}$, respectively. The black solid, red dashed, green dot-dashed, blue dotted, and cyan triple-dot-dashed curves denote $f_{A D A F}$ at $r=2 M$, $4 M, 6 M, 15 M$, and $30 M$, respectively. The results do not depend on the BH mass, because the radius is normalized by the gravitational radius, $r_{\mathrm{g}}=M$. In each panel, the abscissa indicates the photon momentum projected along the radial outward direction; thus, +1 means radially outward propagation while -1 does radially inward one. It follows that the photon intensity decreases with decreasing $\theta$, because most of the photons have positive angular momentum $\lambda$ and hence find it difficult to approach the rotation axis, $\theta=0$. The solid curves (at $r=2 M$ ) in each panel show that the radiation field becomes predominantly inward near the horizon due to the causality, where the horizon is located at $r_{\mathrm{H}}=1.435 \mathrm{M}$ when $a=0.9 \mathrm{M}$. However, at larger radius $r$, the radiation field becomes outwardly unidirectional and its flux decreases by the $r^{-2}$ law, as the blue (at $\left.r=15 M\right)$ and cyan 
(at $r=30 \mathrm{M}$ ) curves indicate. This is because the ADAF photons are emitted only within $r_{\text {out }}=10 \mathrm{M}$ in the present consideration.

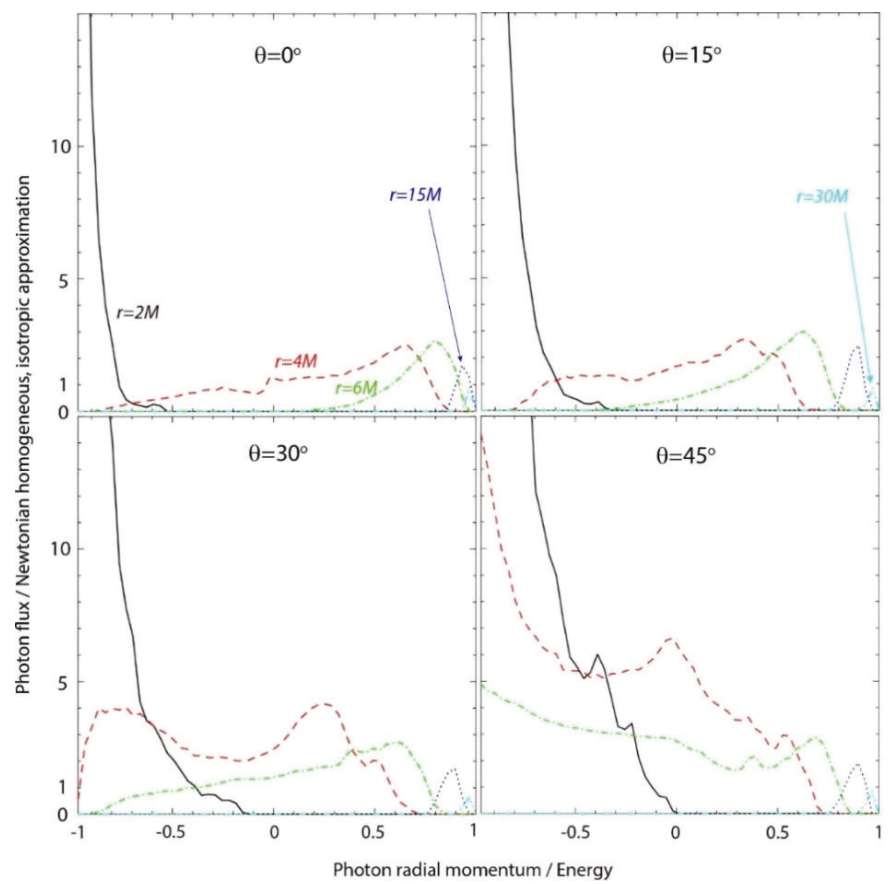

Figure 10. Advection-dominated accretion flow (ADAF) photon flux correction factor, $f_{A D A F}$, as a function of the cosine of the propagation direction, $\cos \theta_{\gamma}$ ZAMO, with respect to the radially outward direction, at five discrete radial positions at three latitudes, as labeled. $\mathrm{BH}$ spin is assumed to be $0.9 \mathrm{M}$. The black solid, red dashed, green dot-dashed, blue dotted, and cyan triple-dot-dashed curves denote $f_{A D A F}$ at $r=2 M, 4 M, 6 M, 15 M$, and $30 M$, respectively. In each panel, the abscissa changes from $\cos \theta_{\gamma} \mathrm{ZAMO}=-1$ to $+1 ;-1$ corresponds to the radially inward direction, whereas +1 does radially outward direction in the zero-angular-momentum observer (ZAMO). From [63].

\subsection{The Poisson Equation in the Tortoise Coordinate}

To solve the radial dependence of $\Phi$ in the Poisson Equation (34), we introduce the following dimensionless tortoise coordinate,

$$
\frac{d \eta_{*}}{d r}=\frac{r^{2}+a^{2}}{\Delta} \frac{1}{M}
$$

In this coordinate, the horizon corresponds to the "inward infinity," $\eta_{*} \rightarrow-\infty$. In this paper, we set $\eta_{*}=r / M$ at $r=25 M$. Note that the function $\eta_{*}=\eta_{*}(r / M)$ does not depend on $\theta$. For a graph of $\eta_{*}=\eta_{*}(r / M)$, see Figure 2 of [62].

Using the tortoise coordinate, the Poisson Equation (34) can be cast into the form

$$
\begin{gathered}
\frac{A}{\Sigma^{2}}\left(\frac{r^{2}+a^{2}}{\Delta}\right)^{2} \frac{\partial^{2} \widetilde{\Phi}}{\partial \eta_{*}^{2}}+\left[\frac{A}{\Sigma^{2}} \frac{2 r\left(a^{2}-M r\right)}{\Delta^{2}}+\frac{r^{2}+a^{2}}{\Sigma \Delta} \frac{\partial}{\partial r}\left(\frac{A}{\Sigma}\right)\right] \frac{\partial \widetilde{\Phi}}{\partial \eta_{*}} \\
+\frac{A}{\Sigma^{2} \Delta} \frac{\partial^{2} \widetilde{\Phi}}{\partial \theta^{2}}+\frac{1}{\Sigma \Delta \sin \theta} \frac{\partial}{\partial \theta}\left(\frac{A \sin \theta}{\Sigma}\right) \frac{\partial \widetilde{\Phi}}{\partial \theta}=\frac{B\left(\eta_{*}, \theta\right)}{B_{\mathrm{H}}}\left[\int\left(n_{+}-n_{-}\right) d \gamma-n_{G J}\right]
\end{gathered}
$$

The $\csc \theta$ factor in the $\partial_{\theta}$ term can be eliminated if we change the variable such as $z=\cos \theta$. 
The dimensionless lepton distribution functions per magnetic flux tube are defined by

$$
n_{ \pm}\left(\eta_{*}, \theta ; \gamma\right) \equiv \frac{2 \pi c e}{\omega_{\mathrm{H}} B\left(\eta_{*}, \theta\right)} N_{ \pm}\left(\eta_{*}, \theta ; \gamma\right)
$$

whereas the dimensionless GJ number density is defined by:

$$
n_{\mathrm{GJ}}\left(\eta_{*}, \theta\right) \equiv \frac{2 \pi c}{\omega_{\mathrm{H}} B\left(\eta_{*}, \theta\right)} \rho_{\mathrm{GJ}}\left(\eta_{*}, \theta\right),
$$

$N_{ \pm}$denotes the distribution function of positrons and electrons in ordinary definition. We have introduced the dimensionless non-corotational potential,

$$
\widetilde{\Phi}\left(\eta_{*}, \theta\right) \equiv \frac{c}{2 M^{2} \omega_{\mathrm{H}} B_{\mathrm{H}}} \Phi(r, \theta),
$$

For a radial poloidal magnetic field, $A_{\varphi}=A_{\varphi}(\theta)$, we can compute the acceleration electric field by

$$
E_{\|} \equiv \frac{\partial \Phi}{\partial r}=\frac{2 M \omega_{\mathrm{H}} B}{c} \frac{r^{2}+a^{2}}{\Delta} \frac{\partial \widetilde{\Phi}}{\partial \eta_{*}},
$$

Without loss of any generality, we can assume $F_{\theta \varphi}>0$ in the northern hemisphere. In this case, a negative $E_{\|}$arises in the gap, which is consistent with the direction of the global current flow pattern.

\subsection{Boltzmann Equations for Electrons and Positrons}

Next, let us describe the lepton Boltzmann equations. Imposing a stationary condition, $\partial_{t}+$ $\Omega_{F} \partial_{\varphi}=0$, we obtain the Boltzmann equations,

$$
\cos \chi \frac{\partial n_{ \pm}}{\partial s}+\dot{p} \frac{\partial n_{ \pm}}{\partial p}=\alpha\left(S_{\mathrm{IC}, \pm}+S_{\mathrm{p}, \pm}\right),
$$

where $\chi$ denotes the pitch angle (with $\chi=0$ for out-going leptons and $\chi=\pi$ for in-coming ones), $s$ and $p$ show the position and dimensionless momentum along the magnetic field, $S_{\text {IC }}$ denotes the rate of positrons or electrons transferred into Lorentz factor $\gamma$ from another Lorentz factor via IC scatterings, $S_{\mathrm{p}}$ refers to that which appeared into Lorentz factor $\gamma$ via photon-photon and magnetic pair production, and $\alpha$ refers to the lapse function, or equivalently, the gravitational redshift factor. In the Boyer-Lindquist coordinate, we obtain

$$
\alpha=\frac{\rho_{w}}{\sqrt{g_{\varphi \varphi}}}=\sqrt{\frac{\Delta \Sigma}{A}}
$$

It follows that $\alpha=0$ at the horizon and $\alpha=1$ at infinity. We consider both photon-photon and magnetic pair production process; however, only the former contributes in all the cases we consider. The upper and lower sign of $\mathrm{n}, S_{\mathrm{IC}}$, and $S_{\mathrm{p}}$ corresponds to the positrons (with charge $q=+e$ ) and electrons $(q=-e)$, respectively. The dimensionless momentum is related to the Lorentz factor by $p \equiv|\mathbf{p}|=m_{e} c \sqrt{\gamma^{2}-1}$, where $m_{\mathrm{e}}$ shows the electron rest mass. The left-hand side is in $d t$ basis, where $t$ denotes the proper time of a distant static observer. Thus, the lapse $\alpha$ is multiplied in the right-hand side, because both $S_{\mathrm{IC}}$, and $S_{\mathrm{p}}$ are evaluated in ZAMO. It is convenient to include the curvature emission as a friction term in the left-hand side. In this case, we obtain:

$$
\dot{p} \equiv q E_{\|} \cos \chi-\frac{P_{\mathrm{SC}}}{c},
$$

where the curvature radiation force is given by (e.g., [119]), $P_{\mathrm{SC}} / c=2 e^{2} \gamma^{4} /\left(3 R_{\mathrm{c}}{ }^{2}\right)$. The IC and pair-production redistribution functions are given in $[50,63]$. 
It is noteworthy that the charge conservation ensures that the dimensionless total current density (per magnetic flux tube),

$$
j_{\text {tot }}(s)=\int\left[-n_{+}(s, \gamma)-n_{-}(s, \gamma)\right] d \gamma,
$$

is conserved along the flowline, where electrons migrate outwards while positron inwards. If we denote the created current density as $J_{\mathrm{cr}}$, the injected current density across the inner and outer boundaries as $J_{\text {in }}$ and $J_{\mathrm{out}}$, respectively, and the typical GJ value as $J_{\mathrm{GJ}}=\omega_{\mathrm{H}} B_{\mathrm{H}} / 2 \pi$, we obtain $j_{\text {tot }}=j_{\text {cr }}+j_{\text {in }}+j_{\text {out }}$, where $j_{\text {cr }} \equiv J_{\text {cr }} / J_{\mathrm{GJ}}, j_{\text {in }} \equiv J_{\text {in }} / J_{\mathrm{GJ}}, j_{\text {out }} \equiv J_{\text {out }} / J_{\mathrm{GJ}}$.

\subsection{Radiative Transfer Equation}

We assume that all photons are emitted with vanishing angular momenta and hence propagate on a constant $\theta$ cone. Under this assumption of radial propagation, we obtain the radiative transfer equation:

$$
\frac{d I_{\omega}}{d l}=-\alpha_{\omega} I_{\omega}+j_{\omega}
$$

where $d l=\sqrt{g_{r r}} d r$ refers to the distance interval along the ray in ZAMO, $\alpha_{\omega}$ and $j_{\omega}$ the absorption and emission coefficients evaluated in ZAMO, respectively. We consider only photon-photon collisions for absorption, pure curvature and IC processes for primary lepton emissions, and synchrotron and IC processes for the emissions by secondary and higher-generation pairs. For more details of the computation of absorption and emission, see Sections 4.2 and 4.3 of [61] and Section 5.1 .5 of [62].

\subsection{Boundary Conditions}

The elliptic type second-order partial differential Equation (49) is solved on the 2-D poloidal plane. We assume a reflection symmetry, $\partial_{\theta} \Phi=0$, at $\theta=0$. We assume that the polar funnel is bounded at a fixed colatitude, $\theta=\theta_{\max }$ and that this lower-latitude boundary is equi-potential and put $\Phi=0$ at $\theta=\theta_{\max }=60^{\circ}$. Both the outer and inner boundaries are treated as free boundaries. At both inner and outer boundaries, $E_{\|}=-\partial \Phi / \partial r$ vanishes. To solve the Boltzmann Equation (54), we need to specify the particle injection rate across the inner and outer boundaries. Since the magnetospheric current is to be constrained by a global condition including the distant dissipative region, we should treat $j_{\mathrm{cr}}, j_{\text {in }}$ and $j_{\text {out }}$ as free parameters, when we focus on the local gap electrodynamics. For simplicity, we assume that there is no electron injection across the inner boundary and put $j_{\text {in }}=0$ throughout this paper. In what follows, we examine stationary gap solutions for several representative values of $j_{\mathrm{cr}}$ and $j_{\text {out }}$. The radiative-transfer Equation (58), a first-order ordinary differential, contains no photon injection across neither the outer nor the inner boundaries.

\subsection{Gap Closure Condition}

We compute the multiplicity (Equation (41) of [61]) of primary electrons, $M_{\text {out }}$, and that of primary positrons, $M_{\text {in }}$, summing up all the created pairs by individual test particles and dividing the result by the number of test particles. With this modification, we apply the same closure condition that a stationary gap may be sustained, $M_{\text {out }} M_{\text {in }}=1$.

\section{Lepton Accelerator around Stellar-Mass Black Holes}

Before we come to the main subject, the case of super-massive BHs, it is desirable to investigate stellar-mass BHs, because important gap electrodynamics have so far been elucidated in this case $[67,68]$.

\subsection{Black Hole Accretion in a Gaseous Cloud}

When a BH moves in an ambient medium, accretion takes place onto the $\mathrm{BH}$. Since the temperature is very low in a molecular cloud, the BH's velocity $V$ becomes supersonic with respect to the gaseous cloud. For a uniform medium, particles within the impact parameter $r_{\mathrm{B}} \approx G M / V^{2}$ will be captured by 
the BH with the hydrodynamical accretion rate $[120,121] \dot{M}_{\mathrm{B}}=4 \pi \lambda_{\mathrm{B}}(G M)^{2} \rho V^{-3}$, where we obtain $\lambda_{B}=1.12$ for an isothermal gas, and 0.25 for an adiabatic one; $\rho$ denotes the mass density of the gas. Thus, we obtain the dimensionless Bondi accretion rate:

$$
\dot{m}_{\mathrm{B}}=5.39 \times 10^{-9} \lambda_{\mathrm{B}} n_{\mathrm{H}_{2}}\left(\frac{M}{10 M_{\odot}}\right)\left(\frac{\eta}{0.1}\right)^{-1}\left(\frac{V}{10^{2} \mathrm{~km} \mathrm{~s}^{-1}}\right)^{-3},
$$

for a molecular hydrogen gas, where $n_{\mathrm{H}_{2}}$ denotes the density of $\mathrm{H}_{2}$ in the unit of $\mathrm{cm}^{-3}, \eta \sim 0.1$ designates the radiation efficiency of the accretion flow. Representative values of $\dot{m}_{\mathrm{B}}$ are plotted as the five straight lines in Figure 11a. Since the accreting gases have little angular momentum as a whole with respect to the $\mathrm{BH}$, they form an accretion disk only within a radius that is much less than the Bondi radius $r_{\mathrm{B}}$. Thus, we neglect the mass loss as a disk wind between $r_{\mathrm{B}}$ and the inner-most region, and evaluate the accretion rate near the $\mathrm{BH}, \dot{m}$ with $\dot{m}_{\mathrm{B}}$. In Section 6 , we consider a 10-solar-mass $\mathrm{BH}$, which is typical as a stellar-mass BH $[122,123]$.

\subsection{Lepton Accelerator around Stellar-Mass Black Holes}

In a vacuum, rotating magnetosphere, an acceleration electric field, $E_{\|}$, inevitably arises. Accordingly, leptons (red arrows in Figure 11b) are accelerated into ultra-relativistic energies to emit high-energy gamma-rays (wavy line with middle wavelength) via the curvature process and VHE gamma-rays (wavy line with shortest wavelength) via the inverse-Compton (IC) scatterings of the soft photons (wavy line with longest wavelength) that were emitted from the ADAF. A fraction of such VHE photons collide with the ADAF soft photons to materialize as $e^{ \pm}$pairs, which partially screen the original $E_{\|}$when they polarize. To compute the actual strength of $E_{\|}$, we solve the $e^{ \pm}$pair production cascade in a stationary and axisymmetric magnetosphere on the meridional plane $(r, \theta)$. The magnetic field lines are twisted into the counter-rotational direction due to the poloidal currents, and the curvature radius of such a toroidal field is assumed to be $r_{\mathrm{g}}=M$ in the local reference frame.

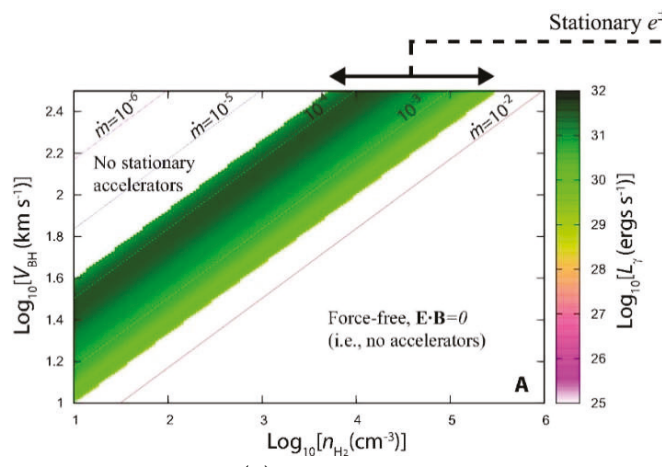

(a)

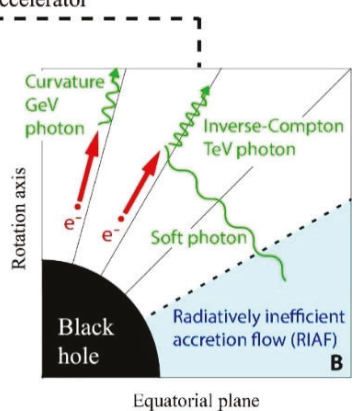

(b)

Figure 11. (a) Luminosity of $\mathrm{BH}$ lepton accelerators when a 10-solar-mass, extremely rotating $(a=0.99 M) \mathrm{BH}$ is moving with velocity $V$ in a cloud whose molecule hydrogen density is $n_{\mathrm{H}_{2}}$. The five straight lines correspond to the Bondi-Hoyle accretion rates, $10^{-2}, 10^{-3}, 10^{-4}, 10^{-5}$, and $10^{-6}$, as labeled. In the lower-right white region, the gap vanishes due to efficient pair production. In the upper-left white region, stationary accelerators cannot be formed. Thus, stationary accelerators arise only in the green-black region. (b) Schematic figure (side view) of a $\mathrm{BH}$ magnetosphere. The polar funnel is assumed to be bounded from the ADAF, a kind of radiatively inefficient accretion flow (RIAF, cyan region) at colatitude $\theta=60 \circ$ (dashed line) from the rotation axis (i.e., the ordinate). From [67]. 


\subsection{Results: Acceleration Electric Field}

To estimate the maximum luminosity of a gap, we consider an extremely rotating case of $a=0.99$ (Figure 4 of [68]). It follows from Figure 12a that $E_{\|}$peaks along the rotation axis, because the magnetic fluxes concentrate towards the rotation axis as $a \rightarrow M$. In what follows, we thus focus on the emission along the rotation axis, $\theta=0^{\circ}$. $E_{\|}$decreases slowly outside the null surface in the same way as pulsar outer gaps (e.g., Figure 12 of [124]). This is due to the two-dimensional screening effect of $E_{\|}$.

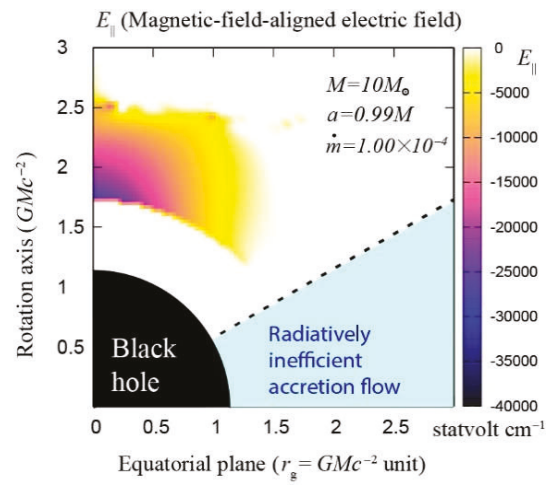

(a)

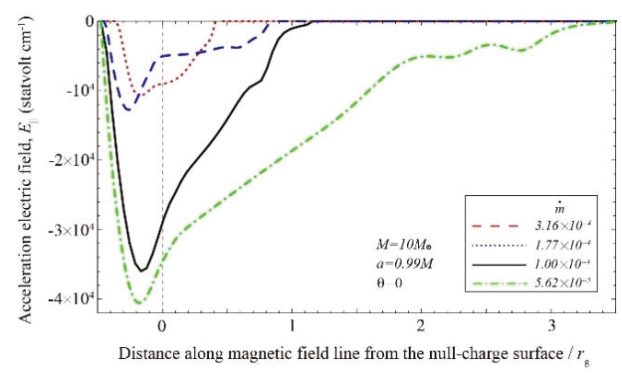

(b)

Figure 12. Acceleration electric field, $E_{\|}$, for $M=10 M_{\odot}, a=0.99 M$, and split-monopole magnetic field lines rotating with angular frequency $\Omega_{\mathrm{F}}=0.5 \omega_{\mathrm{H}}$. (a) Distribution on the poloidal plane; both axes are normalized by the gravitational radius, $r_{\mathrm{g}}$. Strong $E_{\|}$appears only in the polar region. The mass accretion rate is $10^{-4}$ in the Eddington unit. The null-charge surface distributes nearly spherically at radis, $r \approx 1.73 M$. (b) $E_{\|}$as a function of distance from the null surface (abscissa) along the rotation axis. The created current density is $70 \%$ of the Goldreich-Julian value, and the injected currents are set to be zero. The red dashed, blue dotted, green dash-dotted, and black solid curves corresponds to $\dot{m} \equiv \dot{M} / \dot{M}_{\text {Edd }}=3.16 \times 10^{-4}, 1.77 \times 10^{-4}, 1.00 \times 10^{-4}$, and $5.62 \times 10^{-5}$, respectively. The vertical dashed line shows the position of the null-charge surface. From $[67,68]$.

In Figure $12 \mathrm{~b}$, we present $E_{\|}\left(s, \theta=0^{\circ}\right)$ at four dimensionless accretion rates (as indicated in the box). We find that the potential drop increases with decreasing $\dot{m}$. However, if the accretion further decreases as $\dot{m}<10^{-4.25}$, there exists no stationary gap solutions. Below this lower bound accretion rate, the gap becomes inevitably non-stationary (Section 8.1).

\subsection{Results: Ultra-Relativistic Leptons}

Since $E_{\|}<0$, electrons (or positrons) are accelerated outward (or inward), the electrons' Lorentz factors, $\gamma$, gather around $3 \times 10^{6}$ due to the curvature-radiation drag (Figure 2 of [68]). At the same time, $\gamma$ distributes in lower values with a broad plateau in $6 \times 10^{4}<\gamma<2 \times 10^{6}$ due to the IC drag. Since the Klein-Nishina cross section increases with decreasing $\gamma$, such lower-energy electrons with $6 \times 10^{4}<\gamma<2 \times 10^{6}$ efficiently contribute to the VHE emission via IC scatterings [68].

\subsection{Results: Gamma-Ray Spectra}

Let us examine the gamma-ray spectra. In Figure 13a, we present the spectral energy distribution (SED) of the gap emission along five discrete viewing angles. It follows that the gap luminosity maximizes if we observe the $\mathrm{BH}$ almost face-on, $\theta<15^{\circ}$, and that the gap luminosity rapidly decreases at $\theta<30^{\circ}$. 


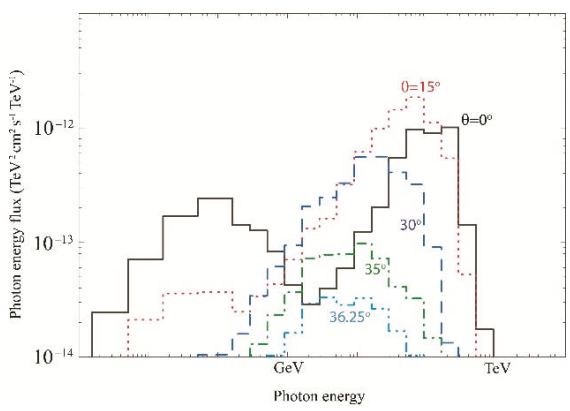

(a)

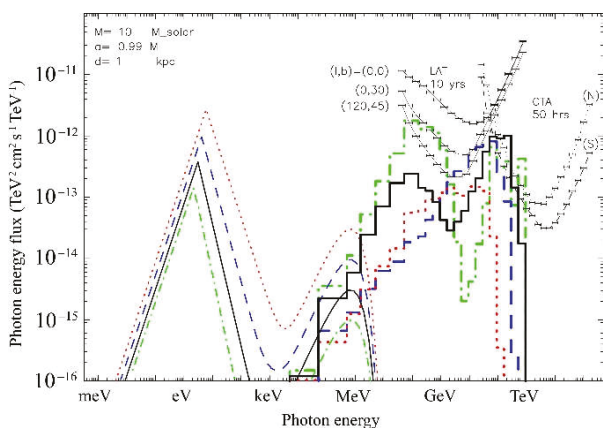

(b)

Figure 13. Spectral energy distribution (SED) of the gap-emitted photon for the BH whose mass and spin are $M=10 M_{\odot}$ and $a=0.99 M$. The created and injected current densities are $j_{\text {cr }}=0.7, j_{\text {in }}=0$, $j_{\text {out }}=0$. (a) SEDs along five discrete viewing angles with respect to the rotation axis; the black solid, red dotted, blue dashed, green dot-dashed, and cyan triple-dot-dashed lines correspond to $\theta=0^{\circ}$, $15^{\circ}, 30^{\circ}, 35^{\circ}$, and $36.25^{\circ}$, respectively. The accretion rate is $\dot{m}=1.00 \times 10^{-4}$. (b) SEDs at four discrete accretion rate; the red dotted, blue dashed, black solid, and green dot-dashed lines correspond to $\dot{m}$ $3.16 \times 10^{-4}, 1.77 \times 10^{-4}, 1.00 \times 10^{-4}$, and $5.62 \times 10^{-5}$, respectively. From $[67,68]$.

In Figure 13b, we show the SEDs for $a=0.99 M$ at $\dot{m}=10^{-3.50}, 10^{-3.75}, 10^{-4.00}$, and $10^{-4.25}$ along $\theta=0^{\circ}$. It follows that the gap luminosity increases with decreasing $\dot{m}$. The VHE flux lies above the CTA detection limit (dashed and dotted curves on the top right), provided that the distance is within $1 \mathrm{kpc}$ and we observe it nearly face on (i.e., $\theta \sim 0^{\circ}$ ). Indeed, the VHE appears above the CTA detection limit if $a>0.90 M$ (Figure 4 [68]). However, if the $\mathrm{BH}$ is moderately rotating as $a=0.50 M$, it is very difficult to detect its emission, unless it is located within $0.3 \mathrm{kpc}$ [68]. It also follows that the spectrum has two peaks. The curvature photons are emitted by the gap-accelerated primary electrons and appear between $5 \mathrm{MeV}$ and $0.5 \mathrm{GeV}$. The same electrons up-scatter the ambient soft photons, forming the second peak between $0.5 \mathrm{GeV}$ and $5 \mathrm{GeV}$. The latter IC photons suffers absorption above $0.1 \mathrm{TeV}$ (Figure 5 of [68]).

\subsection{Results: Dependence on the Current Created in the Gap}

Let us demonstrate that the gap solution exists in a wide range of the created electric current within the gap, $j_{\mathrm{cr}} \equiv J_{\mathrm{cr}} / J_{\mathrm{GJ}}$, and that the resultant gamma-ray spectrum little depends on $j_{\mathrm{cr}}$. In Figure 14, we show the solved $E_{\|}(s)$ (left panels) and SEDs (right panels) for three discrete $j_{\mathrm{cr}}$ 's: from the top, they correspond to $j_{\mathrm{cr}}=0.3,0.5$, and 0.9. The case of $j_{\mathrm{cr}}=0.7$ is presented as Figure $13 \mathrm{~b}$. It is clear that the gap spectra modestly depend on the created current within the gap as long as the created current is sub-GJ. There exist no stationary gap solutions if $j_{\mathrm{cr}}>1$. Note that the maximum value of $\left|E_{\|}\right|$ saturates below $4.5 \times 10^{5}$ statvolt $\mathrm{cm}^{-1}$ because of the $2 \mathrm{D}$ screening effect, which becomes important when the gap width become comparable to or greater than the transverse thickness $\sim M(3.4)$.

It can also be shown that the SEDs depend little on the injected current across the outer boundary (Figure 7 of [68]). For further details, including the authors' comments to a criticism raised by [77] regarding the locations of stationary gaps and the stagnation surface, see Section 6.2 of [68]. 

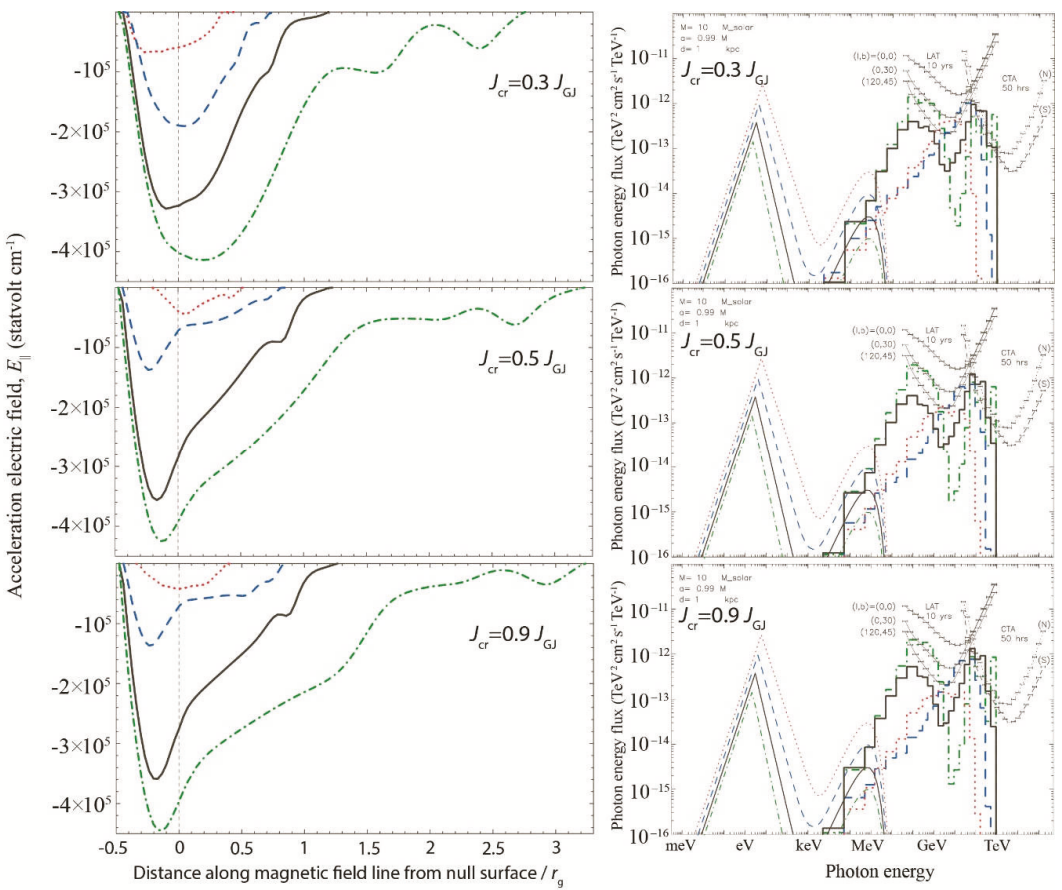

Figure 14. Dependence of the magnetic field-aligned electric fields $E_{\|}(s)$ (left panels) and the SEDs (right panels) on the created current within the gap. From the top, the created current density is 30\%, $50 \%$, and $90 \%$ of the GJ value. In all the three cases, the injected current density across the inner or outer boundaries is set to be zero. The curves corresponds to the accretion rate of $\dot{m}=3.16 \times 10^{-4}$ (red dotted), $1.77 \times 10^{-4}$ (blue dashed), $1.00 \times 10^{-4}$ (black solid), and $5.62 \times 10^{-5}$ (green dash-dotted) From [68].

\section{Lepton Accelerator around Super-Massive Black Holes}

In this section, we apply the BH gap model to supermassive BHs.

\subsection{Very High-Energy Observations of Active Galaxies}

The High-Energy Stereoscopic System (H.E.S.S.), the Major Atmospheric Gamma Imaging Cherenkov (MAGIC), and the Very Energetic Radiation Imaging Telescope Array System (VERITAS) have so far detected $219 \mathrm{TeV}$ sources [125]. Among them, 70 sources have been identified as AGNs. Although most of them are blazer type, NGC 1275, 3C264, M87, and Cen A are classified as Fanaroff-Riley (FR) I radio galaxies. In addition, another radio galaxy, IC 310 appears to be of a transitional type between FR I and BL Lac. For these five non-blazer radio galaxies, their inner jet (on parsec or sub-parsec scales) are moderately misaligned with respect to the line of sight. Thus, their $\mathrm{TeV}$ emission from the jets will not be highly Doppler-boosted. Accordingly, the emission from the direct vicinity of the central supermassive $\mathrm{BH}$, if it exists, may not be hidden by the strong jet emissioin. We are, therefore, motivated to study these non-blazer radio galaxies, comparing theoretical predictions with VHE observations. See Rieger and Levinson [126] for a comprehensive review on the VHE observations of these non-blazer radio galaxies, and associated theoretical arguments.

From NGC 1275, a TeV flare has been reported by MAGIC [127] with the shortest variation time scale of $\Delta t_{\mathrm{var}} \sim 10 \mathrm{~h}$. Since the light crossing time of the event horizon is about $3 \times 10^{3} \mathrm{~s}$ for this source, the dimension of the $\mathrm{TeV}$ emitting region should be less than 10 times Schwarzschild radii. 
M87 exhibited rapidly varying VHE flares that are observed in 2008 and 2010 [128-131], where the shortest variation time scale was observed by all the three IACTs, and was found to be $\Delta t_{\mathrm{var}} \sim 1$ day [128]. Its horizon-crossing time scale, $4 \times 10^{4} \mathrm{~s}$, indicates that the $\mathrm{TeV}$ emitting region should be less than a few Schwarzschild radii. Also, VHE photons were detected during the other periods, including the low-emission state [132-134]. Results of multi-wavelength observations of M87 were summarized in [135].

IC 310 showed very rapid variabilities whose shortest time scale attained $\Delta t_{\mathrm{var}} \sim 5 \mathrm{~m}=300 \mathrm{~s}$, which was detected by MAGIC [77]. Compared with its horizon-crossing time scale of $3 \times 10^{3} \mathrm{~s}$, we find that the $\mathrm{TeV}$ photons should be emitted from a sub-horizon length scale, presumably from the direct vicinity of the event horizon. For a comparison of representative emission models for such rapidly varying extragalactic sources in the TeV sky, see [136].

In the rest of this review, we consider the $\mathrm{BH}$ gap model as a possible explanation of such horizon-scale or sub-horizon-scale TeV flares observed from M87 and IC 310.

\subsection{Results: Acceleration Electric Field}

To investigate the VHE emission from the direct vicinity of a supermassive $\mathrm{BH}$, we apply the BH gap model, and comapre the results obtained for $M=10^{9} \mathrm{M}_{\odot}$. and $10^{6} \mathrm{M}_{\odot}$. Unless explicitly mentioned, we adopt $a=0.90 M, \Omega_{\mathrm{F}}=0.5 \omega_{\mathrm{H}}, j_{\text {tot }}=0.7, j_{\text {in }}=j_{\text {out }}=0$, and the curvature radius is $R_{C}$ $=M$ (due to toroidal bending of the magnetic field lines). To solve the Poisson Equation (49), we set the meridional boundary at $60^{\circ}$.

Let us begin with the $\mathrm{E}_{\|}$distribution on the poloidal plane $\left(\eta_{*}, \theta\right)$, where $\eta_{*} \equiv r_{*} / M$ denotes the dimensionless tortoise coordinate (Section 5.4). In Figure 15, we plot $E_{\|}$(in statvolt cm${ }^{-1}$ ) for billion solar-mass BH (left panel) and for million solar-mass BH (right panel). For both panels, a common accretion rate, $\dot{m}=1.77 \times 10^{-5}$, is adopted. Near the lower-latitude boundary, $\theta=60^{\circ}$, a small $E_{\|}$extends into higher altitudes, because a smaller $E_{\|}$requires a greater width for the gap closure condition to be satisfied. This behavior is common with pulsar lower-altitude slot-gap models $[137,138]$. However, near the rotation axis, $\theta=0^{\circ}$, stronger $E_{\|}$arises, mainly because the polar region is far from the meridional boundary at $\theta=60^{\circ}$, and partly because the magentic fluxes more or less concentrate toward the rotation axis even for a non-extreme rotator with $a=0.90 M$. The strong $E_{\|}$distributes relatively widely in the polar region within $\theta \leq 15^{\circ}$. $E_{\|}$decreases with increasing $\mathrm{BH}$ mass, because the GJ charge density, $\rho_{\mathrm{GJ}} \approx \Omega B / 2 \pi c$, decreases with increasing $\mathrm{BH}$ mass, where $B=B_{\mathrm{eq}}$ (Equation $(1))$.

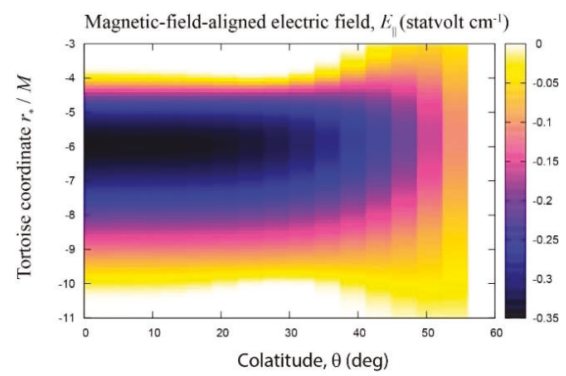

(a)

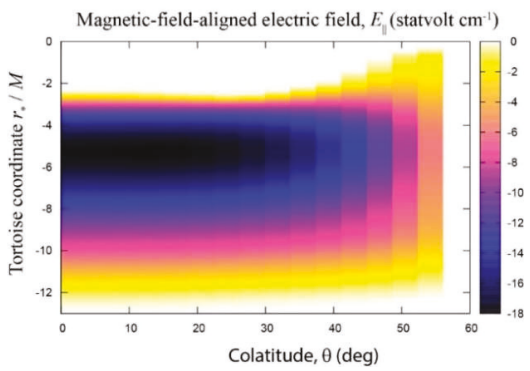

(b)

Figure 15. Acceleration electric field (statvolt $\mathrm{cm}^{-1}$ ) on the poloidal plane. The abscissa denotes the magnetic colatitudes, $\theta$, in degrees, where 0 (i.e., the ordinate) corresponds to the magnetic axis. The ordinate denotes the dimensionless tortoise coordinate, $\eta_{*}=r_{*} / r_{g}$, where $-\infty$ corresponds to the event horizon. BH spin is $a=0.9 M$, and the accretion rate is set at $\dot{m}=1.77 \times 10^{-5}$. (a) The case of $M=$ $10^{9} \mathrm{M}_{\odot}$. (b) The case of $M=10^{6} \mathrm{M}_{\odot}$. From [68]. 


\subsection{Results: Gap Width}

Let us briefly examine how the gap width, $\mathrm{w}$, depends on the ADAF soft-photon field as the accretion rate changes. In Figure 16, we plot the gap inner and outer boundary positions as a function of $\dot{m}$, where the ordinate is converted into the dimensionless Boyer-Lindquist radial coordinate, $r / M$. The left panel shows the result in the case of a billion solar-mass BH, while the right panel does that in the case of a million solar-mass BH. It follows that the gap inner boundary (solid curve, $r=r_{1}$ ) infinitesimally approaches the horizon (dot-dashed horizontal line, $r=r_{\mathrm{H}}$ ), while the outer boundary (dashed curve, $\mathrm{r}=\mathrm{r}_{2}$ ) moves outward, with decreasing $\dot{m}$. At greater accretion rate, $\dot{m}>1.8 \times 10^{-4}$ (for $\mathrm{M}=10^{9} \mathrm{M}_{\odot}$ case), and $\dot{m}>10^{-3}$ (for $M=10^{6} \mathrm{M}_{\odot}$ case), we fail to find stationary solutions. At smaller accretion rate, $\left(\dot{m}<5.6 \times 10^{-6}\right.$ for $M=10^{9} \mathrm{M}_{\odot}$ case, and $\dot{m}<2.3 \times 10^{-5}$ for $M=10^{6} \mathrm{M}_{\odot}$ case), there is no stationary gap solution, because the pair creation becomes too inefficient to create the externally imposed current density, $j_{\text {tot }}$, even with $w » M$. Note that $j_{\text {tot }}$ (along each magnetic flux tube) should be constrained by a global requirement (including the dissipative region at large distances) and cannot be solved if we consider only the gap region.

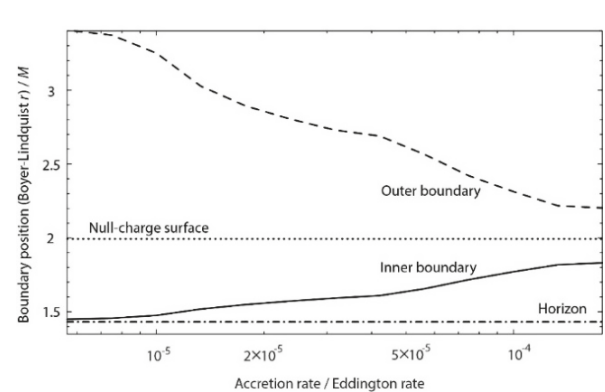

(a)

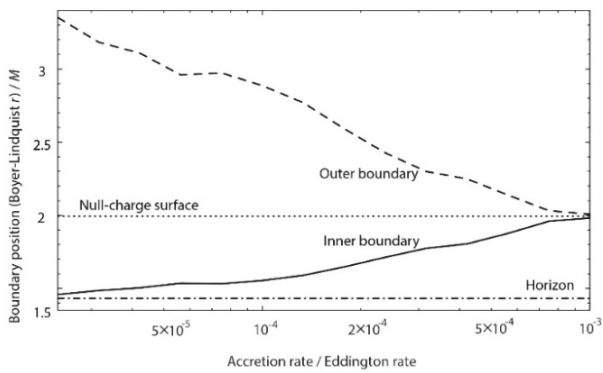

(b)

Figure 16. Spatial extent of the gap along the polar axis, $\theta=0^{\circ}$, as a function of the dimensionless accretion; $a=0.9 \mathrm{M}$ is assumed. Thick solid and dashed curves denote the position of the inner and the outer boundaries of the gap, respectively, in units of the gravitational radius, $r_{\mathrm{g}}=M$. These boundary positions (in the ordinate) are transformed from the tortoise coordinate into the Boyer-Lindquist coordinate. The horizontal dot-dashed line shows the horizon radius, whereas the horizontal dotted line shows the null surface position. (a) The case of $M=10^{9} \mathrm{M}_{\odot}$. (b) The case of $M=10^{6} \mathrm{M}_{\odot}$. From [68].

\subsection{Results: Distribution Function of Electrons}

Because $E_{\|}$is negative, electrons are accelerated outward while positrons inward. Thus, the outward $\gamma$-rays, which we observe, are emitted by the electrons. Therefore, we focus on the distribution function of the electrons created inside the gap. In Figure 17, we plot the electron distribution function, $\gamma n_{-}(r, \gamma)$, along the rotation axis, $\theta=0^{\circ}$, when the accretion rate is $\dot{m}=1.77 \times 10^{-5}$. Note that $\gamma n_{-}$ denotes the electron phase space density per logarithmic Lorentz factor. The abscissa denotes the Lorentz factor, $\gamma$, in the logarithmic scale. The ordinate denotes the distance $s$ along the magnetic field line from the null surface and converted into the Boyer-Lindquist radial coordinate. Note that $s \approx r-r_{0}$ holds near the rotation axis. Electrons migrate outward from the lower part of this figure to the upper part. In the case of a billion solar-mass BH (left panel), the accelerated electrons lose energy via IC scatterings and distribute between $4 \times 10^{7}<\gamma<1.5 \times 10^{8}$. Near and outside the outer boundary, the decelerated electrons become nearly monoenergetic, forming a "shock" in the phase space (due to the concentration of their characteristics) at $r-r_{0}>0.60 \mathrm{M}$. In the case of a million solar-mass BH (right panel), electron's distribution function has two peaks in the outer half of the gap, $r-r_{0}>0.40 \mathrm{M}$. The lower-energy peak appears in $10^{7.2}<\gamma<10^{7.4}$ because of the radiation drag due to IC scatterings. Below $10^{7.2}$, IC scatterings take place in the Thomson regime; thus, the scattering 
cross section is almost unchanged, while energy transfer per scattering decreases with decreasing $\gamma$ by $\gamma^{2}$. Accordingly, the IC radiation drag works less effectively compared to the Klein-Nishina regime, in which a substantial fraction of the electron's energy is transferred to the photon. Thus, $\gamma n_{-}$ peaks slightly above $\gamma \sim 10^{7.2}$. The higher-energy peak appears in $10^{7.85}<\gamma<10^{8.25}$, because the lepton Lorentz factors saturate in this range owing to the curvature radiation drag. Unlike the stellar-mass cases (§6), however, the curvature component contributes only mildly for supermassive BHs even when $\dot{m}$ approaches its lower bound ( $\dot{m}=2 \times 10^{-5}$ in the present case), below which a stationary gap ceases to exist. For the graph of the electron distribution functions at several positions in the gap, see [68].

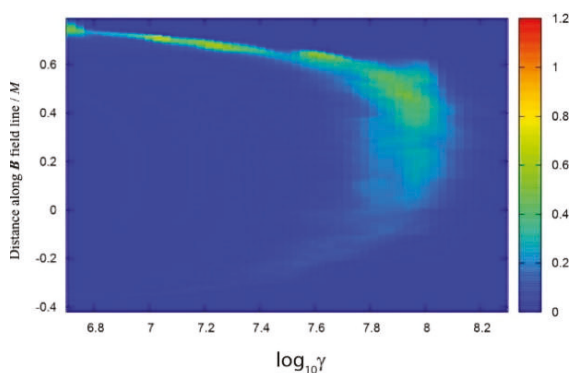

(a)

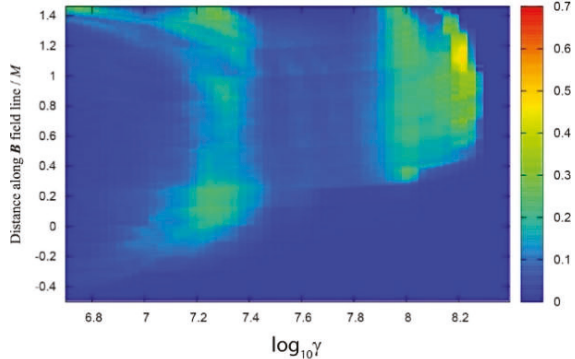

(b)

Figure 17. Electron distribution function, $\gamma n_{-}(r, \gamma)$, of the electrons along the magnetic field line on the polar axis, $\theta=0^{\circ}$, for $a=0.9 \mathrm{M}$ and $\dot{m}=1.77 \times 10^{-5}$. The abscissa denotes the electron Lorentz factor, while the ordinate denotes the distance from the null surface, $s \approx r-r_{0}$, along the poloidal magnetic field line, where the ordinate is converted into the Boyer-Lindquist radial coordinate. (a) The case of $M$ $=10^{9} \mathrm{M}_{\odot}$. (b) The case of $M=10^{6} \mathrm{M}_{\odot}$. From [63].

\subsection{Results: Gamma-Ray Spectra}

The predicted photon spectra are depicted in Figure 18 for six $m$ values. The thin curves on the left denote the input ADAF spectra, while the thick lines on the right denote the output spectra from the gap. The left panel shows the result in the case of a billion solar-mass $\mathrm{BH}$ assuming the distance of $d=10 \mathrm{Mpc}$, while the right panel does that in the case of a million solar-mass $\mathrm{BH}$ with $d=1 \mathrm{Mpc}$. In both cases, the emitted $\gamma$-ray flux increases with decreasing $\dot{m}$, because the decreased soft photon field increases the photon-photon collision mean free path, and hence the gap width.

In the case of $\mathrm{M}=10^{9} \mathrm{M}_{\odot}$ (left panel), the spectra peak between 1 and $30 \mathrm{TeV}$, because the IC process dominates the curvature one. It is clear that the gap HE flux lies well below the detection limit of the Fermi/LAT (three thin solid curves labeled with "LAT 10 year" [139]). Nevertheless, its VHE flux appears above the CTA detection limits (dashed and dotted curves labeled with "CTA 50 h" [140]). Is is possible to argue that such a large VHE flux will be detected within one night with CTA from a nearby low-luminosity like M87, when the accretion rate coincidentally resides in the range, $6 \times 10^{-6}$ $<\dot{m}<3 \times 10^{-5}$.

In the case of $\mathrm{M}=10^{6} \mathrm{M}_{\odot}$ (right panel), the spectra peak between $30 \mathrm{GeV}$ and $10 \mathrm{TeV}$. The IC process dominates the curvature one also for million solar-mass BHs. When the accretion rate is in the narrow range $2 \times 10^{-5}<\dot{m}<3 \times 10^{-5}$, the gap emission may be detectable with CTA, particularly if the source is located in the southern sky. 


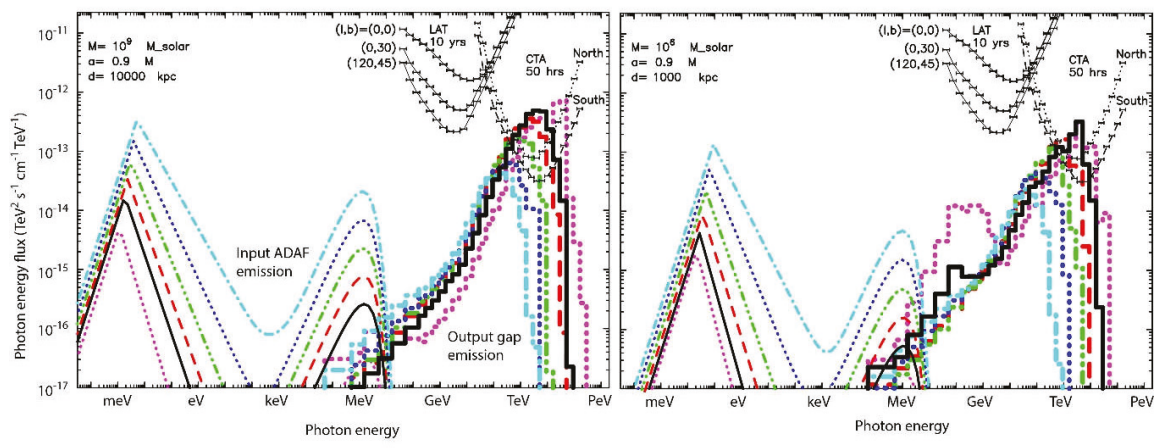

(a)

(b)

Figure 18. SED of the gap emission for a supermassive BH with $a=0.9 M$. The thin curves denote the input ADAF spectra, while the thick lines denote the output gap spectra. The thin solid curves (with horizontal bars) denote the Fermi/LAT detection limits after 10 year of observation, while the thin dashed and dotted curves (with horizontal bars) denote the CTA detection limits after $50 \mathrm{~h}$ of observation. (a) The case of $M=10^{9} \mathrm{M}_{\odot}$. The distance is set to be $10 \mathrm{Mpc}$. The cyan dot-dashed, blue dotted, green triple-dot-dashed, red dashed, black solid, and purple dotted curves correspond to $\dot{m}=1.77 \times 10^{-4}, 1.00 \times 10^{-4}, 5.62 \times 10^{-5}, 3.16 \times 10^{-5}, 1.77 \times 10^{-5}$, and $5.62 \times 10^{-6}$, respectively. Magnetic field strength is assumed to be the equipartition value with the plasma accretion. (b) The case of $\mathrm{M}=10^{6} \mathrm{M}_{\odot}$. The distance is set to be $1 \mathrm{Mpc}$. The cyan dot-dashed, blue dotted, green triple-dot-dashed, red dashed, black solid, and purple dotted curves correspond to $m=3.16 \times 10^{-4}$, $1.00 \times 10^{-4}, 5.62 \times 10^{-5}, 4.21 \times 10^{-5}, 3.16 \times 10^{-5}$, and $1.77 \times 10^{-5}$, respectively. From [63].

\section{Discussion}

\subsection{The Case of Very Small Accretion Rate}

Let us discuss the case of very small accretion rates, $\dot{m}<\dot{m}_{\text {low }}(M, a)$. The ADAF photon field peaks around $\mathrm{eV}$ for stellar-mass $\mathrm{BHs}$ and around $\mathrm{meV}$ for supermassive $\mathrm{BHs}$. These photons are emitted from the innermost region, $r \sim R_{\min } \sim 6 M$, which is located well inside the gap outer boundary when $\dot{m} \approx \dot{m}_{\text {low }}$; thus, pair production is sustained only marginally in an extended gap. However, at $\dot{m}<\dot{m}_{\text {low }}$, stationary pair production can be no longer sustained and a vacuum region develops in the entire polar funnel. In this vacuum region, migratory leptons are accelerated by the vacuum $E_{\|}$and cascade into copious primary electrons and positrons that are accelerated in the opposite directions. The resultant emission will become inevitably non-stationary.

\subsection{Gap Emission Versus Jet Emission}

Next, let us discuss how to discriminate the gap and jet emissions. As we have seen in Sections 6 and 7 , the gap gamma-ray luminosity increases with decreasing $\dot{m}$. Thus, we can predict an anti-correlation between the ADAF-emitted infrared (IR)/optical and the gap-emitted HE/VHE fluxes for stellar-mass BHs, and between the ADAF-emitted radio and the gap-emitted VHE fluxes for supermassive BHs. This forms a contrast to the standard shock-in-jet scenario, whose natural prediction will be a correlation between the radio/IR/optical and the HE/VHE fluxes (for reviews or catalogues of gamma-ray observations of AGNs, see e.g., [126,141-145]). This anti-correlation or correlation can be detectable both for stellar-mass and supermassive BHs. For stellar-mass BHs, BH transients may exhibit a correlation between IR/optical and HE/VHE fluxes during quiescence or low-hard state. For supermassive BHs, nearby low-luminosity AGNs may show an anti-correlation between submillimeter wavelength and VHE. In X-rays, the gap emission is very weak. Thus, if X-ray photons are detected, they are probably emitted from the jet or from the accretion flow. 


\subsection{Comparison with Pulsar Gap Models}

Let us compare the present BH gap model with the pulsar outer (OG) gap model. In both gap models, the gap appears around the null-charge surface where the GJ charge density vanishes, as the stationary solution of the Maxwell-Boltzmann equations. Moreover, the gap width, and hence the $\gamma$-ray efficiency, which is defined by the ratio between the $\gamma$-ray and spin-down luminosities, increases with decreasing soft-photon flux. This is because the pair-production mean-free path increases with decreasing photon flux. For instance, the OG $\gamma$-ray efficiency increases with decreasing NS surface emission, or equivalently with increasing NS age $[45,146]$. In the same manner, the BH gap $\gamma$-ray efficiency increases with decreasing accretion rate of ADAF. In addition, electron-positron pairs are supplied by photon-photon pair production in both pulsar and BH magnetosphere, although ions can be drawn into the pulsar OG from the neutron-star surface as a space-charge limit flow [147], in the same manner as in PC models (2).

However, there are differences, as described below.

First, in a pulsar magnetosphere, the null surface appears because of the convex geometry of a dipole magnetic field. On the other hand, in a BH magnetosphere, a null surface appears due to the frame-dragging effect around a rotating $\mathrm{BH}$. Thus, for the same magnetic field polarity, the sign of $E_{\|}$ is opposite.

Second, in the OG model, a soft-photon field is provided by the cooling NS thermal emission and/or the heated polar-cap thermal emission. The NS surface emissions peak in the X-ray energies. Thus, the curvature 1-10 GeV photons efficiently materialize as pairs within the gap, thereby contributing to the gap closure. However, the thermal IR photon field is much weaker than the thermal X-ray field; thus, the IC photons do not contribute to the gap closure in pulsars. On the other hand, around a $\mathrm{BH}$ it is the ADAF that provides the soft-photon field. For a supermassive $\mathrm{BH}$, the ADAF spectrum peaks in submillimeter wavelengths. Thus, the photons having energies around $100 \mathrm{TeV}$ or above efficiently materialize as pairs to close the gap. The $0.1-10 \mathrm{GeV}$ curvature photons, on the other hand, do not materialize efficiently because the IR photon number flux is five to six orders of magnitude weaker than the submillimeter one.

Let us briefly compare the BH gap model with the pulsar polar-cap (PC) model. In a pulsar magnetosphere, electrons may be drawn outward as a space-charge-limited flow at the NS surface in the PC region. Thus, in a stationary gap [31,32] or in a non-stationary gap [34], $\gamma$-ray emission could be realized without pair creation within the polar cap, although pairs are indeed created via magnetic pair creation (e.g., at least at the outer boundary where $E_{\| \mid}$should be screened). However, in BH magnetospheres, causality prevents any plasma emission across the horizon. Thus, a gap could not be sustained without pair creation.

If we compare the stationary BH gap model with pulsar OG and PC models, it is possible to argue for the stability of gaps in a qualitative manner ( $\$ 6.3$ of [68]). In this review, finally we discuss the fact that the non-stationary nature of pulsar PCs [34] cannot readily be extended to the stability arguments of stationary BH gap solutions. In a pulsar PC, there exists no null surface. Thus, if a three-dimensional PC region is charge-starved in the sense $\left|\rho-\rho_{\mathrm{GJ}}\right| \ll\left|\rho_{\mathrm{GJ}}\right|$, a positive $-\rho_{\mathrm{GJ}}$ leads to a negative $E_{\|}$. Consider a small-amplitude pair production taking place near the upper boundary of a PC accelerator. Created positrons migrate inwards while electrons outwards, resulting in an increased (or decreased) $\rho-\rho_{\mathrm{GJ}}$ in the lower (or the upper) part of the pair-production site. Accordingly, $\left|E_{\|}\right|$increases (or decreases) in the lower part (or the upper part). The increased $\left|E_{\|}\right|$in the lower part further enhances pair production in the upper regions, because the electrons drawn from the neutron star surface will gain more energy. As a result, $\left|E_{\|}\right|$, and hence the pair production increases with time. Because of this positive feedback effect, instability sets in, as demonstrated by PIC simulations [34]. In short, pulsar PC accelerators are inherently unstable for pair production because of $E_{\|}<0$, which stems from the fact that there exists no null-charge surface in the PC region due to the relatively weak frame-dragging effect (i.e., the Lense-Thirring effect [148]) around a rotating neutron star. 
On these grounds, we cannot readily conclude that the stationary BH gap solutions are unstable because of the highly time-dependent nature of the pulsar PC accelerators. Careful examinations with PIC simulations are needed for BH gaps, as performed recently by $[81,83]$. Since the saturated solution in recent PIC simulation is much less violently time-dependent compared to pulsar PC cases [34,81], it may be reasonable to start with stationary $\mathrm{BH}$ gap models as the first step.

Funding: This research received no external funding.

Acknowledgments: The author is indebted to H.-Y. Pu for providing the computed spectral data of advection-dominated accretion flows, which are used in Sections 6 and 7.

Conflicts of Interest: The author declares no conflict of interest.

\section{References}

1. Ferrarese, L.; Ford, H.C.; Jaffe, W. Evidence for a Massive Black Hole in the Active Galaxy NGC 4261 from Hubble Space Telescope Images and Spectra. Astrophys. J. 1996, 470, 444-459. [CrossRef]

2. Larkin, A.C.; McLaughlin, D.E. Dark-matter haloes and the M- $\sigma$ relation for supermassive black holes. Mon. Not. R. Astron. Soc. 2016, 462, 1864-1881. [CrossRef]

3. Van den Bosch, R.C.E. Unification of the fundamental plane and super massive black hole masses. Astrophys. J. 2016, 831, 134. [CrossRef]

4. Bentz, M.C.; Manne-Nicholas, E. Black hole-galaxy scaling relationships for Active Galactic Nuclei with reverberation masses. Astrophys. J. 2018, 864, 146. [CrossRef]

5. Kormendy, J.; Richstone, D. Inward Bound-The Search for Supermassive Black Holes in Galactic Nuclei. Annu. Rev. Astron. Astrophys. 1995, 33, 581-624. [CrossRef]

6. Kormendy, J.; Ho, L.C. Coevolution (or not) of supermassive black holes and host galaxies. Annu. Rev. Astron. Astrophys. 2013, 51, 511-653. [CrossRef]

7. Miyoshi, M.; Moran, J.; Herrnstein, J.; Greenhill, L.; Nakai, N.; Diamond, P.; Inoue, M. Evidence for a black hole from high rotation velocities in a sub-parsec region of NGC4258. Nature 1995, 373, 127-129. [CrossRef]

8. Mogorrian, J.; Tremaine, S.; Richstone, D.; Bender, R.; Bower, G.; Dressler, A.; Faber, S.M.; Gebhardt, K.; Green, R.; Grillmair, C.; et al. The Demography of Massive Dark Objects in Galaxy Centers. Astron. J. 1998, 115, 2285-2305. [CrossRef]

9. Ferrarese, L.; Merritt, D. A Fundamental Relation between Supermassive Black Holes and Their Host Galaxies. Astrophys. J. Lett. 2000, 539, L9-L12. [CrossRef]

10. Gebhardt, K.; Bender, R.; Bower, G. A Relationship between Nuclear Black Hole Mass and Galaxy Velocity Dispersion. Astrophys. J. 2000, 539, L13-L16. [CrossRef]

11. Bentz, M.C.; Katz, S. The AGN black hole mass database. Publ. Astron. Soc. Pac. 2015, 127, 67-73. [CrossRef]

12. Lynden-bell, D. Galactic Nuclei as Collapsed Old Quasars. Nature 1969, 223, 690-694. [CrossRef]

13. Blandford, R.D.; Znajek, R.L. Electromagnetic extraction of energy from Kerr black holes. Mon. Not. R. Astron. Soc. 1977, 179, 433-456. [CrossRef]

14. Misner, C.W.; Thorne, K.S.; Wheeler, J.A. Gravitation; W.H. Freeman: San Francisco, CA, USA, 1973; p. 875.

15. Meier, D.L.; Koide, S.; Uchida, U. Magnetohydrodynamic Production of Relativistic Jets. Science 2001, 291, 84-92. [CrossRef] [PubMed]

16. Hujeirat, A. A model for electromagnetic extraction of rotational energy and formation of accretion-powered jets in radio galaxies. Astron. Astrophys. 2004, 416, 423-435. [CrossRef]

17. Sadowski, A.; Sikora, M. Launching of jets by cold, magnetized disks in Kerr metric. Astron. Astrophys. 2010, 517, A18. [CrossRef]

18. Koide, S.; Shibata, K.; Kudoh, T.; Meier, D.L. Extraction of black hole rotational energy by a magnetic field and the formation of relativistic jets. Science 2002, 295, 1688-1691. [CrossRef] [PubMed]

19. McKinney, J.C. General relativistic magnetohydrodynamic simulations of the jet formation and large-scale propagation from black hole accretion systems. Mon. Not. R. Astron. Soc. 2006, 368, 1561-1582. [CrossRef]

20. McKinney, J.C.; Tchekhovskoy, A.; Blandford, R.D. General relativistic magnetohydrodynamic simulations of magnetically choked accretion flows around black holes. Mon. Not. R. Astron. Soc. 2012, 423, 3083-3117. [CrossRef] 
21. Punsly, B. Evidence on the origin of ergospheric disc field line topology in simulations of black hole accretion. Mon. Not. R. Astron. Soc. 2011, 418, L138-L142. [CrossRef]

22. Hirose, S.; Krolik, J.; de Villiers, J.-P.; Hawley, J.F. Magnetically Driven Accretion Flows in the Kerr Metric. II. Structure of the Magnetic Field. Astrophys. J. 2004, 606, 1083-1097. [CrossRef]

23. McKinney, J.C.; Gammie, C.F. A Measurement of the Electromagnetic Luminosity of a Kerr Black Hole. Astrophys. J. 2004, 611, 977-995. [CrossRef]

24. Tchekhovskoy, A.; Narayan, R.; McKinney, J.C. Black Hole Spin and the Radio Loud/Quiet Dichotomy of Active Galactic Nuclei. Astrophys. J. 2010, 711, 50-63. [CrossRef]

25. Hawley, J.F.; Krolik, J.H. Magnetically Driven Jets in the Kerr Metric. Astrophys. J. 2006, 641, $103-116$. [CrossRef]

26. McKinney, J.C.; Narayan, R. Disc-jet coupling in black hole accretion systems-I. General relativistic magnetohydrodynamical models. Mon. Not. R. Astron. Soc. 2007, 375, 513-530. [CrossRef]

27. McKinney, J.C.; Narayan, R. Disc-jet coupling in black hole accretion systems-II. Force-free electrodynamical models. Mon. Not. R. Astron. Soc. 2007, 375, 531-547. [CrossRef]

28. Ichimaru, S. Bimodal behavior of accretion disks - Theory and application to Cygnus X-1 transitions. Astrophys. J. 1977, 214, 840-855. [CrossRef]

29. Narayan, R.; Yi, I. Advection-dominated accretion: A self-similar solution. Astrophys. J. 1994, 428, L13-L16. [CrossRef]

30. Public List of LAT-Detected Gamma-Ray Pulsars. Available online: https:/ / confluence.slac.stanford.edu/ display / GLAMCOG/Public+List+of+LAT-Detected+Gamma-Ray+Pulsars (accessed on 21 November 2018).

31. Harding, A.K.; Tademaru, E.; Esposito, L.S. A curvature-radiation-pair-production model for gamma-ray pulsars. Astrophys. J. 1978, 225, 226-236. [CrossRef]

32. Daugherty, J.K.; Harding, A.K. Electromagnetic cascades in pulsars. Astrophys. J. 1982, 252, 337-347. [CrossRef]

33. Dermer, C.D.; Sturner, S.J. On the energetics and number of gamma-ray pulsars. Astrophys. J. 1994, 420, L75-L78. [CrossRef]

34. Timokhin, A.N.; Arons, J. Current flow and pair creation at low altitude in rotation-powered pulsars' force-free magnetospheres: Space charge limited flow. Mon. Not. R. Astron. Soc. 2013, 429, 20-54. [CrossRef]

35. Timokhin, A.N.; Harding, A.K. On the Polar Cap Cascade Pair Multiplicity of Young Pulsars. Astrophys. J. 2015, 810, 144. [CrossRef]

36. Cheng, A.F.; Ruderman, M.A.; Sutherland, P.G. Current flow in pulsar magnetospheres. Astrophys. J. 1976, 203, 209-212. [CrossRef]

37. Cheng, K.S.; Ho, C.; Ruderman, M. Energetic radiation from rapidly spinning pulsars. I—Outer magnetosphere gaps. Astrophys. J. 1986, 300, 500-521. [CrossRef]

38. Cheng, K.S.; Ho, C.; Ruderman, M. Energetic radiation from rapidly spinning pulsars. II. Vela and Crab. Astrophys. J. 1986, 300, 522-539. [CrossRef]

39. Chiang, J.; Romani, R.W. Gamma radiation from pulsar magnetospheric gaps. Astrophys. J. 1992, 400, $629-637$. [CrossRef]

40. Romani, R. Gamma-ray pulsars: Radiation processes in the outer magnetosphere. Astrophys. J. 1996, 470, 469-478. [CrossRef]

41. Cheng, K.S.; Ruderman, M.; Zhang, L. A Three-dimensional Outer Magnetospheric Gap Model for Gamma-Ray Pulsars: Geometry, Pair Production, Emission Morphologies, and Phase-resolved Spectra. Astrophys. J. 2000, 537, 964-976. [CrossRef]

42. Takata, J.; Shibata, S.; Hirotani, K. A pulsar outer gap model with trans-field structure. Mon. Not. R. Astron. Soc. 2004, 354, 1120-1132. [CrossRef]

43. Hirotani, K. High-energy emission from pulsar magnetospheres. Mod. Phys. Lett. A Brief Rev. 2006, 21, 1319-1337. [CrossRef]

44. Romani, R.; Watters, K.P. Constraining Pulsar Magnetosphere Geometry with $\gamma$-ray Light Curves. Astrophys. J. 2010, 714, 810-824. [CrossRef]

45. Hirotani, K. Luminosity Evolution of Gamma-Ray Pulsars. Astrophys. J. 2013, 766, 98. [CrossRef]

46. Takata, J.; Ng, C.W.; Cheng, K.S. Probing gamma-ray emissions of Fermi-LAT pulsars with a non-stationary outer gap model. Mon. Not. R. Astron. Soc. 2016, 455, 4249-4266. [CrossRef] 
47. Vigano, D.; Torres, D.F.; Hirotani, K.; Pessah, M.E. An assessment of the pulsar outer gap model.-I. Assupmtions, uncertainties, and implications on the gap size and the accelerating field. Mon. Not. R. Astron. Soc. 2015, 447, 2631-2648. [CrossRef]

48. Cheng, K.S.; Zhang, L. General Radiation Formulae for a Relativistic Charged Particle Moving in Curved Magnetic Field Lines: The Synchrocurvature Radiation Mechanism. Astrophys. J. 1996, 463, 271-283. [CrossRef]

49. Vigano, D.; Torres, D.F.; Hirotani, K.; Pessah, M.E. Compact formulae, dynamics and radiation of charged particles under synchro-curvature losses. Mon. Not. R. Astron. Soc. 2015, 447, 1164-1172. [CrossRef]

50. Hirotani, K.; Harding, A.K.; Shibata, S. Electrodynamics of an Outer Gap Accelerator: Formation of a Soft Power-Law Spectrum between $100 \mathrm{MeV}$ and $3 \mathrm{GeV}$. Astrophys. J. 2003, 591, 334-353. [CrossRef]

51. Reville, B.; Kirk, J. Linear acceleration emission in pulsar magnetospheres. Astrophys. J. 2010, 715, $186-193$. [CrossRef]

52. Beskin, V.S.; Istomin, Y.N.; Par'ev, V.I. Filling the Magnetosphere of a Supermassive Black-Hole with Plasma. Soviet Astron. 2002, 36, 642-649.

53. Hirotani, K.; Okamoto, I. Pair Plasma Production in a Force-free Magnetosphere around a Supermassive Black Hole. Astrophys. J. 1998, 497, 563-572. [CrossRef]

54. Neronov, A.; Aharonian, F.A. Production of $\mathrm{TeV}$ gammaradiation in the vicinity of the supermassive black hole in the giant radio galaxy M87. Astrophys. J. 2007, 671, 85-96. [CrossRef]

55. Marconi, A.; Axon, D.J.; Macchetto, F.D.; Cappetti, A.; Sparks, W.B.; Crane, P. Is there really a supermassive black hole in M87? Mon. Not. R. Astron. Soc. 1997, 289, L21-L25. [CrossRef]

56. Macchetto, F.; Marconi, A.; Axon, D.J.; Capetti, A.; Sparks, W.; Crane, P. The supermassive black hole of M87 and the kinematics of its associated gaseous disk. Astrophys. J. 1997, 489, 579-600. [CrossRef]

57. Gebhardt, K.; Adams, J.; Richstone, D.; Lauer, T.R.; Faber, S.M.; Gültekin, K.; Murphy, J.; Tremaine, S. The black hole mass in M87 from Gemini/NIFS adaptive optics observations. Astrophys. J. 2011, 729, 119. [CrossRef]

58. Walsh, J.L.; Barth, A.J.; Ho, C.; Sarzi, M. The M87 Black Hole Mass from Gas-dynamical Models of Space Telescope Imaging Spectrograph Observations. Astrophys. J. 2013, 770, 86. [CrossRef]

59. Levinson, A.; Rieger, F. Variable TeV emission as a manifestation of jet formation in M87? Astrophys. J. 2011, 730, 123. [CrossRef]

60. Broderick, A.E.; Tchekhovskoy, A. Horizon-scale lepton acceleration in jets: Explaining the compact radio emission in M87. Astrophys. J. 2015, 809, 97. [CrossRef]

61. Hirotani, K.; Pu, H.-Y. Energetic gamma radiation from rapidly rotating black holes. Astrophys. J. 2016, 818, 50. [CrossRef]

62. Hirotani, K.; Pu, H.-Y.; Lin, L.C.-C.; Chang, H.-K.; Inoue, M.; Kong, A.K.H.; Matsushita, S.; Tam, P.-H.T. Lepton acceleration in the vicinity of the event horizon: High-energy and very-high-energy emissions from rotating black holes with various masses. Astrophys. J. 2016, 833, 142. [CrossRef]

63. Hirotani, K.; Pu, H.-Y.; Lin, L.C.-C.; Kong, A.K.H.; Matsushita, S.; Asada, K.; Chang, H.-K.; Tam, P.-H.T. Lepton acceleration in the vicinity of the event horizon: Very high energy emissions from supermassive black holes. Astrophys. J. 2017, 845, 77. [CrossRef]

64. Lin, L.C.-C.; Pu, H.-Y.; Hirotani, K.; Kong, A.K.H.; Matsushita, S.; Chang, H.-K.; Inoue, M.; Tam, P.-H.T. Searching for high-energy, horizon-scale emissions from galactic black hole transients during quiescence. Astrophys. J. 2017, 845, 40. [CrossRef]

65. Song, Y.; Pu, H.-Y.; Hirotani, K.; Matsushita, S.; Kong, A.K.H.; Chang, H.-K. Enhanced gamma radiation towards the rotation axis from the immediate vicinity of extremely rotating black holes. Mon. Not. R. Astron. Soc. 2017, 471, L135-L139. [CrossRef]

66. Komissarov, S.S.; McKinney, J.C. The 'Meissner effect' and the Blandford-Znajek mechanism in conductive black hole magnetospheres. Mon. Not. R. Astron. Soc. 2007, 377, L49-L53. [CrossRef]

67. Hirotani, K.; Pu, H.-Y.; Matsushita, S. Lightning black holes as unidentified TeV sources. Astrophys. J. 2018, 39, 50. [CrossRef]

68. Hirotani, K.; Pu, H.-Y.; Outmani, S.; Huang, H.; Kim, D.; Song, Y.; Matsushita, S.; Kong, A.K.H. High-energy and very-high-energy emissions from stellar-mass black holes moving in gaseous clouds. Astrophys. J. 2018, 867, 120. [CrossRef] 
69. Tababe, K.; Nagataki, S. Extended monopole solution of the Blandford-Znajek mechanism: Higher order terms for a Kerr parameter. Phys. Rev. D 2008, 78, 024004.

70. Znajek, R.L. Black hole electrodynamics and the Carter tetrad. Mon. Not. R. Astron. Soc. 1977, 179, 457-472. [CrossRef]

71. Bower, G.C.; Wright, M.C.H.; Falcke, H.; Backer, D.C. Interferometric detection of linear polarization from Sagittarius A* at $230 \mathrm{GHz}$. Astrophys. J. 2003, 588, 331-337. [CrossRef]

72. Marrone, D.P.; Moran, J.M.; Zhao, J.; Rao, R. Interferometric Measurements of Variable 340 GHz Linear Polarization in Sagittarius A*. Astrophys. J. 2006, 640, 308-318. [CrossRef]

73. Macquart, J.-P.; Bower, G.C.; Wright, M.C.H.; Backer, D.C.; Falcke, H. The Rotation Measure and 3.5 Millimeter Polarization of Sagittarius A*. Astrophys. J. 2006, 646, L111-L114. [CrossRef]

74. Kuo, C.Y.; Asada, K.; Rao, R.; Nakamura, M.; Algaba, J.C.; Liu, H.B.; Inoue, M.; Koch, P.M.; Ho, P.T.P.; Matsushita, S.; et al. Measuring Mass Accretion Rate onto the Supermassive Black Hole in M87 Using Faraday Rotation Measure with the Submillimeter Array. Astrophys. J. 2014, 783, L33. [CrossRef]

75. Sijbring, D.; de Bruyn, A.G. Multifrequency radio continuum observations of head-tail galaxies in the Perseus cluster. Astrophys. J. 1998, 331, 901-915.

76. Aleksic, J.; Ansoldi, S.; Antonelli, L.A.; Antoranz, P.; Babic, A.; Bangale, P.; Barrio, J.A.; González, J.B.; Bednarek, W.; Bernardini, E.; et al. Black hole lightning due to particle acceleration at subhorizon scales. Science 2014, 346, 1080-1084. [CrossRef] [PubMed]

77. Levinson, A.; Segev, F. On the existence of steady gap solutions in rotating black hole magnetospheres. PRD 2017, 96, 123006. [CrossRef]

78. Hirotani, K.; Shibata, S. Electrodynamic structure of an outer-gap accelerator: Invisibility of the TeV emission from a pulsar magnetosphere. Publ. Astron. Soc. Jpn. 2000, 52, 645-657. [CrossRef]

79. Kerr, R.P. Gravitational Field of a Spinning Mass as an Example of Algebraically Special Metrics. Phys. Rev. Lett. 1963, 11, 237-238. [CrossRef]

80. Boyer, R.H.; Lindquist, R.W.J. Maximal Analytic Extension of the Kerr Metric. Math. Phys. 1967, 8, $265-281$. [CrossRef]

81. Levinson, A.; Cerutti, B. Particle-in-cell simulations of pair discharges in a starved magnetosphere of a Kerr black hole. Astron. Astrophys. 2018, 616, 184. [CrossRef]

82. Levinson, A.; Melrose, D.; Judge, A.; Luo, Q. Large-Amplitude, Pair-creating Oscillations in Pulsar and Black Hole Magnetospheres. Astrophys. J. 2005, 631, 456-465. [CrossRef]

83. Chen, A.Y.; Yuan, Y.; Yang, H. Physics of Pair Producing Gaps in Black Hole Magnetospheres. Astrophys. J. 2018, 863, L31. [CrossRef]

84. Michel, F.C. Rotating Magnetospheres: An Exact 3-D Solution. Astrophys. J. 1973, 180, L133-L137. [CrossRef]

85. Krolik, J.H.; Hawley, J.F.; Hirose, S. Magnetically driven accretion flows in the Kerr metric. IV. Dynamical properties of the inner disk. Astrophys. J. 2005, 622, 1008-1023. [CrossRef]

86. Punsly, B.; Igumenshchev, I.V.; Hirose, S. Three-dimensional simulations of vertical magnetic flux in the immediate vicinity of black holes. Astrophys. J. 2009, 704, 1065-1085. [CrossRef]

87. Rees, M.J.; Begelman, M.C.; Blandford, R.D.; Phinney, E.S. Ion-supported tori and the origin of radio jets. Nature 1982, 295, 17-21. [CrossRef]

88. Narayan, R.; Yi, I. Advection-dominated Accretion: Underfed Black Holes and Neutron Stars. Astrophys. J. 1995, 452, 710-735. [CrossRef]

89. Abramowicz, M.A.; Chen, X.; Kato, S.; Lasota, J.-P.; Regev, O. Thermal equilibria of accretion disks. Astrophys. J. 1995, 438, L37-L39. [CrossRef]

90. Mahadevan, R. Scaling Laws for Advection-dominated Flows: Applications to Low-Luminosity Galactic Nuclei. Astrophys. J. 1997, 477, 585-601. [CrossRef]

91. Manmoto, T. Advection-dominated Accretion Flow around a Kerr Black Hole. Astrophys. J. 2000, 534, 734-746. [CrossRef]

92. Yuan, F.; Narayan, R. Hot Accretion Flows Around Black Holes. Annu. Rev. Astron. Astrophys. 2014, 52, 529-588. [CrossRef]

93. Mahadevan, R. Reconciling the spectrum of Sagittarius $A^{*}$ with a two-temperature plasma model. Nature 1998, 394, 651-653. [CrossRef]

94. Özel, F.; Psaltis, D.; Narayan, R. Hybrid Thermal-Nonthermal Synchrotron Emission from Hot Accretion Flows. Astrophys. J. 2000, 541, 234-249. [CrossRef] 
95. Yuan, F.; Quataert, E.; Narayan, R. Nonthermal Electrons in Radiatively Inefficient Accretion Flow Models of Sagittarius A*. Astrophys. J. 2003, 598, 301-312. [CrossRef]

96. Liu, H.; Wu, Q. Possible Origin of Radio Emission from Nonthermal Electrons in Hot Accretion Flows for Low-luminosity Active Galactic Nuclei. Astrophys. J. 2013, 764, 17. [CrossRef]

97. Bisnovatyi-Kogan, G.S.; Lovelace, R.V.E. Influence of Ohmic Heating on Advection-dominated Accretion Flows. Astrophys. J. Lett. 1997, 486, L43-L46. [CrossRef]

98. Quataert, E.; Gruzinov, A. Turbulence and Particle Heating in Advection-dominated Accretion Flows. Astrophys. J. 1999, 520, 248-255. [CrossRef]

99. Ding, J.; Yuan, F.; Liang, E. Electron Heating and Acceleration by Magnetic Reconnection in Hot Accretion Flows. Astrophys. J. 2010, 708, 1545-1550. [CrossRef]

100. Hoshino, M. Stochastic Particle Acceleration in Multiple Magnetic Islands during Reconnection. Phys. Rev. Lett. 2012, 108, 135003. [CrossRef] [PubMed]

101. Hoshino, M. Particle Acceleration during Magnetorotational Instability in a Collisionless Accretion Disk. Astrophys. J. 2013, 773, 118. [CrossRef]

102. Quataert, E. Particle Heating by Alfvénic Turbulence in Hot Accretion Flows. Astrophys. J. 1998, 500, $978-991$. [CrossRef]

103. Blackman, E.G. On particle energization in accretion flows. Mon. Not. R. Astron. Soc. 1999, 302, 723-730. [CrossRef]

104. Medvedev, M.V. Particle Heating by Nonlinear Alfvénic Turbulence in Advection-dominated Accretion Flows. Astrophys. J. 2000, 541, 811-820. [CrossRef]

105. Lehe, R.; Parrish, I.J.; Quataert, E. The Heating of Test Particles in Numerical Simulations of Alfvénic Turbulence. Astrophys. J. 2009, 707, 404-419. [CrossRef]

106. Sharma, P.; Quataert, E.; Hammett, G.W.; Stone, J.M. Electron Heating in Hot Accretion Flows. Astrophys. J. 2007, 667, 714-723. [CrossRef]

107. Camenzind, M.A. Centrifugally driven MHD-winds in active galactic nuclei. Astron. Astrophys. 1986, 156, 137-151.

108. Camenzind, M.A. Hydromagnetic flows from rapidly rotating compact objects. I-Cold relativistic flows from rapid rotators. Astron. Astrophys. 1986, 162, 32-44.

109. Bogovalov, S.V. On the physics of cold MHD winds from oblique rotators. Astron. Astrophys. 1999, 349, 1017-1026.

110. Pètri, J.; Kirk, J.G. The Polarization of High-Energy Pulsar Radiation in the Striped Wind Model. Astrophys. J. Lett. 2005, 627, L37-L40. [CrossRef]

111. Pètri, J. Phase-resolved polarization properties of the pulsar striped wind synchrotron emission. Mon. Not. R. Astron. Soc. 2013, 434, 2636-2644. [CrossRef]

112. Hirotani, K.; Shibata, S. Electrodynamic structure of an outer gap accelerator: Location of the gap and the gamma-ray emission from the Crab pulsar. Astrophys. J. 2001, 558, 216-227. [CrossRef]

113. Beskin, V.S.; Zheltoukhov, A.A. On the structure of the magnetic field near a black hole in active galactic nuclei. AstL 2013, 39, 215-220. [CrossRef]

114. Sikora, M.; Begelmann, M.C. Magnetic Flux Paradigm for Radio Loudness of Active Galactic Nuclei. Astrophys. J. 2013, 764, L24-L28. [CrossRef]

115. Takahashi, M.; Nitta, S.; Tatematsu, Y.; Tomimatsu, A. Magnetohydrodynamic flows in Kerr geometry-Energy extraction from black holes. Astrophys. J. 1990, 363, 206-217. [CrossRef]

116. Hirotani, K.; Shibata, S. One-dimensional electric field structure of an outer gap accelerator-III. Location of the gap and the gamma-ray spectrum. Mon. Not. R. Astron. Soc. 2001, 325, 1228-1240. [CrossRef]

117. Hirotani, K.; Shibata, S. Gamma-ray emission from an outer gap accelerator: Constraints on magnetospheric current, magnetic inclination, and distance for PSR B1055-52. Astrophys. J. 2002, 564, 369-378. [CrossRef]

118. Li, Y.-R.; Yuan, Y.-F.; Wang, J.-M.; Wang, J.-C.; Zhang, S. Spins of Supermassive Black Holes in M87. II. Fully General Relativistic Calculations. Astrophys. J. 2009, 699, 513-524. [CrossRef]

119. Harding, A.K. Pulsar gamma-rays - Spectra, luminosities, and efficiencies. Astrophys. J. 1981, 245, $267-273$. [CrossRef]

120. Bondi, H. On spherically symmetrical accretion. Mon. Not. R. Astron. Soc. 1952, 112, 195. [CrossRef]

121. Bondi, H.; Hoyle, F. On the mechanism of accretion by stars. Mon. Not. R. Astron. Soc. 1944, 104, $273-282$. [CrossRef] 
122. Tetarenko, B.E.; Sivakoff, G.R.; Heinke, C.O.; Gladstone, J.C. WATCHDOG: A Comprehensive All-sky Database of Galactic Black Hole X-ray Binaries. Astrophys. J. Suppl. 2016, 222, 15. [CrossRef]

123. Corral-Santana, J.M.; Casares, J.; Muñoz-Darias, T.; Bauer, F.E.; Martínez-Pais, I.G.; Russell, D.M. BlackCAT: A catalogue of stellar-mass black holes in X-ray transients. Astron. Astrophys. 2016, 587, A61. [CrossRef]

124. Hirotani, K.; Shibata, S. One-dimensional electric field structure of an outer gap accelerator-I. gamma-ray production resulting from curvature radiation. Mon. Not. R. Astron. Soc. 1999, 308, 54-66. [CrossRef]

125. TeVCAT. Available online: http://tevcat.uchicago.edu/ (accessed on 21 November 2018).

126. Rieger, F.M.; Levinson, A. Radio galaxies at VHE energies. arXiv 2018, arXiv:1810.05409v1.

127. Ansoldi, S.; Antonelli, L.A.; Arcaro, C.; Baack, D.; Babić, A.; Banerjee, B.; Bangale, P.; de Almeida, U.B.; Barrio, J.A.; González, J.B.; et al. Gamma-ray flaring activity of NGC 1275 in 2016-2017 measured by MAGIC. Astron. Astrophys. 2018, 617, A91. [CrossRef]

128. Albert, J.; Aliu, E.; Anderhub, H.; Biland, A.; Britvitch, I.; Commichau, S.; Kranich, D.; Lorenz, E.; Pauss, F.; Rissi, M.T.; et al. Very High Energy Gamma-Ray Observations of Strong Flaring Activity in M87 in 2008 February. Astrophys. J. Lett. 2008, 685, L23-L26. [CrossRef]

129. Acciari, V.A.; Aliu, E.; Arlen, T. Radio Imaging of the Very-High-Energy $\gamma$-Ray Emission Region in the Central Engine of a Radio Galaxy. Science 2009, 325, 444-448. [PubMed]

130. Acciari, V.A.; Aliu, E.; Arlen, T.; Aune, T.; Beilicke, M.; Benbow, W.; Boltuch, D.; Bradbury, S.M.; Buckley, J.H.; Bugaev, V.; et al. Veritas 2008-2009 Monitoring of the Variable Gamma-ray Source M87. Astrophys. J. 2010, 716, 819-824. [CrossRef]

131. Aliu, E.; Arlen, T.; Aune, T.; Beilicke, M.; Benbow, W.; Bouvier, A.; Bradbury, S.M.; Buckley, J.H.; Bugaev, V.; Byrum, K.; et al. VERITAS observations of day-scale flaring of M87 in 2010 April. Astrophys. J. 2012, 746, 141. [CrossRef]

132. Aharonian, F.; Akhperjanian, A.G.; Bazer-Bachi, A.R.; Beilicke, M.; Benbow, W.; Berge, D.; Bernlöhr, K.; Boisson, C.; Bolz, O.; Borrel, V.; et al. Fast Variability of Tera-Electron Volt $\gamma$ Rays from the Radio Galaxy M87. Science 2006, 314, 1424-1427. [CrossRef] [PubMed]

133. Acciari, V.A.; Beilicke, M.; Blaylock, G.; Bradbury, S.M.; Buckley, J.H.; Bugaev, V.; Butt, Y.; Celik, O.; Cesarini, A.; Ciupik, L.; et al. Observation of Gamma-Ray Emission from the Galaxy M87 above 250 GeV with VERITAS. Astrophys. J. 2008, 679, 397-403. [CrossRef]

134. Aleksić, J.; Alvarez, E.A.; Antonelli, L.A.; Antoranz, P.; Asensio, M.; Backes, M.; Barrio, J.A.; Bastieri, D.; Gonzalez, J.B.; Bednarek, W.; et al. MAGIC observations of the giant radio galaxy M 87 in a low-emission state between 2005 and 2007. Astron. Astrophys. 2012, 544, A96. [CrossRef]

135. Abramowski, A.; Acero, F.; Aharonian, F.; Akhperjanian, A.G.; Anton, G.; Balzer, A.; Barnacka, A.; de Almeida, U.B.; Becherini, Y.; Becker, J.; et al. The 2010 very high energy gamma-ray flare and 10 years of multi-wavelength observations of M87. Astrophys. J. 2012, 746, 151. [CrossRef]

136. Aharonian, F.A.; Barkov, M.V.; Khangulyan, D. Scenarios for ultrafast gamma-ray variability in AGN. Astrophys. J. 2017, 841, 61. [CrossRef]

137. Arons, J. Pair creation above pulsar polar caps: Geometrical structure and energetics of slot gap. Astrophys. J. 1983, 266, 215-241. [CrossRef]

138. Muslimov, A.G.; Harding, A.K. Extended acceleration in slot gaps and pulsar hig-energy emission. Astrophys. J. 2003, 588, 430-440. [CrossRef]

139. Available online: http://www.slac.stanford.edu/exp/glast/groups/canda/lat_Performance.htm (accessed on 21 November 2018).

140. Available online: https://www.cta-observatory.org/science/cta-performance/ (accessed on 21 November 2018).

141. Ackermann, M.; Ajello, M.; Atwood, W.B.; Baldini, L.; Ballet, J.; Barbiellini, G.; Bastieri, D.; Becerra Gonzalez, J.; Bellazzini, R.; Bissaldi, E.; et al. The Third Catalog of Active Galactic Nuclei Detected by the Fermi Large Area Telescope. Astrophys. J. 2015, 810, 14. [CrossRef]

142. Ackermann, M.; Ajello, M.; Atwood, W.B.; Baldini, L.; Ballet, J.; Barbiellini, G.; Bastieri, D.; Becerra Gonzalez, J.; Bellazzini, R.; Bissaldi, E.; et al. 2FHL: The Second Catalog of Hard Fermi-LAT Sources. Astrophys. J. 2016, 222, 5. [CrossRef]

143. Abdollahi, S.; Ackermann, M.; Ajello, M.; Albert, A.; Baldini, L.; Ballet, J.; Barbiellini, G.; Bastieri, D.; Becerra Gonzalez, J.; Bellazzini, R.; et al. The second catalog of flaring gamma-ray sources from the Fermi all-sky variability analysis. Astrophys. J. 2017, 846, 34. [CrossRef] 
144. Rani, B. Radio galaxies-the TeV challenge. Available online: https://arxiv.org/abs/1811.00567 (accessed on 5 November 2018).

145. Madejski, G.; Sikora, M. Gamma-ray observations of active galactic nuclei. Annu. Rev. Astron. Astroph. 2016, 54, 725-760. [CrossRef]

146. Abdo, A.A.; Ajello, M.; Allafort, A.; Baldini, L.; Ballet, J.; Barbiellini, G.; Baring, M.G.; Bastieri, D.; Belfiore, A.; Bellazzini, R.; et al. The second FERMI LARGE AREA TELESCOPE catalog of gamma-ray pulsars. Astrophys. J. Suppl. 2013, 208, 17. [CrossRef]

147. Hirotani, K. Particle acceleration in pulsar magnetospheres: Super-Goldreich-Julian current with ion emission from the neutron star surface. Astrophys. J. 2006, 652, 1475-1493. [CrossRef]

148. Lense, J.; Thirring, H. Ueber den Einfluß der Eigenrotation der Zentralkoerper auf die Bewegung der Planeten und Monde nach der Einsteinschen Gravitationstheorie. Physikalische Zeitschrift 1984, 19, 156-163; Translation in Gener. Relativ. Gravit. 1984, 16, 727-741.

(C) 2018 by the author. Licensee MDPI, Basel, Switzerland. This article is an open access article distributed under the terms and conditions of the Creative Commons Attribution (CC BY) license (http:/ / creativecommons.org/licenses/by/4.0/). 


\title{
Dissipative Processes and Their Role in the Evolution of Radio Galaxies
}

\author{
Manel Perucho ${ }^{1,2}$ \\ 1 Departament d'Astronomia i Astrofísica, Universitat de València, C/Dr. Moliner, 50, \\ 46100 Burjassot, Valencian Country, Spain; manel.perucho@valencia.edu \\ 2 Observatori Astronòmic, Universitat de València, C/Catedràtic José Beltrán 2, \\ 46980 Paterna, Valencian Country, Spain
}

Received: 27 May 2019; Accepted: 29 July 2019; Published: 31 July 2019

\begin{abstract}
Particle acceleration in relativistic jets, to very high levels of energy, occurs at the expense of the dissipation of magnetic or kinetic energy. Therefore, understanding the processes that can trigger this dissipation is key to the characterization of the energy budgets and particle acceleration mechanisms in action in active galaxies. Instabilities and entrainment are two obvious candidates to trigger dissipation. On the one hand, supersonic, relativistic flows threaded by helical fields, as expected from the standard formation models of jets in supermassive black-holes, are unstable to a series of magnetohydrodynamical instabilities, such as the Kelvin-Helmholtz, current-driven, or possibly the pressure-driven instabilities. Furthermore, in the case of expanding jets, the Rayleigh-Taylor and centrifugal instabilities may also develop. With all these destabilizing processes in action, a natural question is to ask how can some jets keep their collimated structure along hundreds of kiloparsecs. On the other hand, the interaction of the jet with stars and clouds of gas that cross the flow in their orbits around the galactic centers provides another scenario in which kinetic energy can be efficiently converted into internal energy and particles can be accelerated to non-thermal energies. In this contribution, I review the conditions under which these processes occur and their role both in jet evolution and propagation and energy dissipation.
\end{abstract}

Keywords: galaxies: active; galaxies: jets; X-rays: binaries; relativistic processes ISM: jets and outflows; magnetohydrodynamics; radiation mechanisms: non-thermal

\section{Introduction}

Relativistic jets and outflows are a common feature in radio-emitting active galaxies, associated with accretion processes onto supermassive black-holes (SMBH, [1-3]). According to the standard picture of relativistic jet formation, they are formed due to the extraction of rotational energy from the central hole by the magnetic field falling onto it along with the accreting gas [4-7]. After formation in a very compact region of a few Schwarzschild radii $\left(R_{s}=3 \times 10^{13} M_{8} \mathrm{~cm}\right.$, with $M_{8}$ the SMBH mass in units of $10^{8} M_{\odot}$ ), the jets propagate through up to hundreds of kiloparsecs, showing remarkable collimation at large scales in some cases, or plumed and irregular structure in others. A correlation between the total jet radio luminosity and its large-scale morphology was found by Fanaroff and Riley [8], who set a morphological division of radio galaxies between those that have outer regions brighter than their centres, Fanaroff-Riley type II (FRII) radio galaxies, and those that are brighter close to the nucleus (and show decollimation at kiloparsec-scales), Fanaroff-Riley type I (FRI) radio galaxies. The former show collimation up to the interaction site with the ambient medium, where a bright radio emission region, the hotspot, is interpreted as the terminal shock that the jet particles cross. It is important to state that powerful X-ray binaries may share the formation mechanism with powerful radio sources and also present a similar morphology $[9,10]$. 
Jets can dramatically influence the evolution of the galactic environment, and thus probably are crucial piece in the evolution of galaxies and the history of star formation in the universe. Their relativistic propagation speeds make them supersonic, which results in the generation of shocks that propagate through the interstellar medium (ISM), heating them and pushing ithemoutwards, as revealed by the correlation between the radio-lobes and X-ray cavities that appear at kpc-scales in radio galaxies (see, e.g., [11-20], and references therein).

The FRI/FRII morphological dichotomy has been related to different estimates of jet kinetic power, with FRIIs corresponding to more powerful jets and FRIs corresponding to weaker jets. Although jet kinetic power is a difficult magnitude to estimate, this seems to be a solid trend (e.g., [21]), with FRII typical jet powers $L_{j} \geq 10^{45} \mathrm{erg} / \mathrm{s}$, and $L_{j} \leq 10^{44} \mathrm{erg} / \mathrm{s}$ for FRIs. At the transition powers, the ambient medium can play a role in the global morphology and this is probably the region where the hybrid morphology sources can develop, in which one of the jets shows FRI morphology, whereas the second jet is an FRII [22].

The remarkable symmetry observed between jets and counter-jets at the large scales in FRIs was interpreted and modelled as caused by strong deceleration [23-27], as opposed to the expected jet to counter-jet brightness asymmetry in the case of relativistic flows, which is caused by relativistic Doppler boosting. This asymmetry is precisely observed in FRIIs. G. Bicknell [28-32], D. De Young [33,34] and S. Komissarov [35-37] set the basis of the FRI jet evolution paradigm, which is grounded on the transition from a supersonic flow to a transonic, turbulent flow, via entrainment and deceleration. Since then, a large number of works tried to sort out what is the exact physical process that is responsible for jet deceleration in low-luminosity radio galaxies. The proposed scenarios include the development of long-wavelength ( $\lambda \geq R_{j}$, with $R_{j}$ the jet radius) Kelvin-Helmholtz or kink current-driven instabilities (KHI and CDI, respectively, [38-40]), strong recollimation shocks [41], entrainment by stellar winds [42-44], or entrainment via turbulent mixing from the shear-layer between the jet and its surrounding medium $[28,34,45]$. All these processes imply strong dissipation, but they differ in the way this occurs. Long-wavelength instabilities and strong recollimation shocks imply relatively fast dissipation, whereas entrainment caused by small-wavelength instabilities or stellar-winds is a slower process that can thus extend the deceleration and dissipation along longer distances. In $[27,46]$, the authors used the modelling of radio images of several FRI radio galaxies to conclude that the deceleration process is extended (in all but one of the studied cases) and, moreover, coincides with a region in which the jets are X-ray bright. Altogether, this result favors the progressive dissipation scenario.

Independently of their large-scale morphology, the radio galaxies emit radiation across the whole electromagnetic spectrum, also at gamma-rays, as shown by the Fermi Large Area Telescope (see, e.g., [47], and references therein). Interestingly, however, the number of detections is relatively larger for FRIs than for FRIIs, which cannot be explained, unless it is caused by intrinsic differences between the jets of both populations $[48,49]$. It was suggested that this could be due to different beaming and jet structures [48]. Furthermore, it was shown that the gamma-ray emission from the FRI radiogalaxy Centaurus A is extended (not localized at the core, but in the lobes) and diffuse [50]. Again, this requires extended energy dissipation that can be invested in particle acceleration via different mechanisms. Within the jet, both the acceleration at shearing layers [51-59] and at the interaction site with stars or clouds (e.g., [60-66]) were invoked to explain high-energy (HE) and very-high-energy (VHE) from radio galaxies, although at different positions along the jet.

In this contribution, I summarize the physical processes that represent possible ways to dissipate energy in jets, namely the development of instabilities and mass entrainment. I also argue that the conversion of the initial jet kinetic energy into internal energy via shocks and turbulent dissipation at the mixing regions could favor particle acceleration. As a consequence, this could explain, at least to some extent, the diffuse HE emission detected in FRI sources, because these processes are more efficient in the case of decelerating jets. 
The paper is structured as follows: in Section 2, I focus on the instabilities that can arise in relativistic jets, separating the linear and non-linear regime phases and I discuss the possible explanation for the stability of FRII jets as compared to FRIs. Section 3 is devoted to jet-star/cloud interactions. In Section 4, I discuss the global role of stellar mass-load in jet dynamics, and Section 5 includes the discussion, in which I develop the production of high-energy and very-high-energy radiation.

\section{Jet Stability}

\subsection{Why Are Jets Unstable?}

In the same way that a kid tests if something may be broken by trying to break it, the way to know whether a system is unstable is destabilizing it. This can be easily done by introducing a small perturbation in the variables that govern it and linearizing the resulting equations. The equations that we use to model the plasma forming the relativistic jets are the relativistic magnetohydrodynamics (RMHD) equations. The stress-energy tensor for the magnetised plasma is $T^{\mu \nu}=T_{G}^{\mu \nu}+T_{E M^{\prime}}^{\mu \nu}$, with ${ }^{1}$

$$
T_{G}^{\mu v}=\rho h u^{\mu} u^{v}+p g^{\mu v}
$$

where the Greek indices $\mu, v=0,1,2,3$ denote the four dimensions of the Minkowski space-time, $\rho$ is the flow rest-mass density, $h$ the gas specific enthalpy, $u$ is the four-velocity, $p$ is the gas pressure, and $g$ the Minkowski metric, and

$$
T_{E M}^{\mu v}=F_{\beta}^{\mu} F^{\nu \beta}-\frac{1}{4} g^{\mu v} F_{\alpha \beta} F^{\alpha \beta},
$$

with $F^{\alpha \beta}=\epsilon^{\alpha \beta \mu v} b_{\mu} u_{v}$ the elements of the electromagnetic field tensor satisfying the Maxwell equations:

$$
\partial_{\alpha} F_{\mu v}=0 \quad \nabla_{\mu} F^{\mu v}=-J^{v},
$$

where $\epsilon^{\alpha \beta \mu v}$ is the Levi-Civita tensor, $b_{\mu}$ is the magnetic field in the fluid rest-frame, and $J^{v}$ is the charge four-current. This results in the following expression for the stress-energy tensor of a magnetized, perfect fluid:

$$
T^{\mu v}=\rho h^{*} u^{\mu} u^{v}+p^{*} g^{\mu v}-b^{\mu} b^{v},
$$

with $h^{*}=h+|b|^{2} / \rho$ the gas plus field enthalpy, $p^{*}$ the gas plus magnetic pressure $\left(p^{*}=p_{\text {gas }}+|b|^{2} / 2\right)$, and $|b|^{2}=b_{\alpha} b^{\alpha}=B^{2} / \gamma^{2}+(\mathbf{v} \cdot \mathbf{B})^{2}$. Please note that $a \sqrt{4 \pi}$ factor is embedded into the definition of the magnetic fields (and this definition is used throughout the paper).

The equations that dictate the flow dynamics, according to our mathematical modelling are derived from the following conservation laws:

$$
\nabla_{\mu} T^{\mu \nu}=0 \quad \nabla_{\mu}\left(\rho u^{\mu}\right)=0 .
$$

The resulting equations (see [67]) are the conservation of mass

$$
\frac{\partial \gamma \rho}{\partial t}+\nabla_{i}\left(\gamma \rho v^{i}\right)=0
$$

where $\mathbf{v}$ is the three-velocity vector (bold-faced variables express three-vectors) and $\gamma$ is the Lorentz factor. The conservation of momentum,

$$
\frac{\partial \gamma^{2} \rho h^{*} v^{i}}{\partial t}+\nabla_{j}\left(\gamma^{2} \rho h^{*} v^{i} v^{j}+p^{*} \delta^{i j}-b^{i} b^{j}\right)=0
$$

\footnotetext{
1 I will use $c=1$ throughout the paper.
} 
where $i, j=1,2,3$ represent the spatial coordinates, $v^{i}$ are the components of the three-velocity vector, and $b^{i}=B^{i} / \gamma+v^{i} \gamma(\mathbf{v} \cdot \mathbf{B})$ are the spatial components of the magnetic four vector, with $\mathbf{B}$ the magnetic field in the observer's frame. In addition, finally, the energy equation can be obtained from $\nabla_{\mu} T^{\mu 0}=0$ :

$$
\frac{\partial\left(\rho h^{*} \gamma^{2}-p^{*}-b^{0} b^{0}-\rho \gamma\right)}{\partial t}+\nabla \cdot\left(\rho h^{*} \gamma^{2} \mathbf{v}-b^{0} \mathbf{b}-\rho \gamma \mathbf{v}\right)=0
$$

with $b^{0}=\gamma(\mathbf{v} \cdot \mathbf{B})$.

The field equations for ideal MHD are

$$
\frac{\partial \mathbf{B}}{\partial t}+\nabla \times \mathbf{E}=0, \quad \nabla \cdot \mathbf{B}=0 .
$$

where $\mathbf{E}=-\mathbf{v} \times \mathbf{B}$ is the electric field three-vector.

Equations (6)-(9) can be applied to the description of an infinite flow, initially axisymmetric, embedded within a given ambient medium. The next step is to introduce a perturbation in each of the physical variables in the observer's frame, $X$, and linearize the equations (in cylindrical coordinates):

$$
X(r) \longrightarrow X_{0}(r)+X_{1}(r, \phi, z, t),
$$

with $X_{0}(r)$ the equilibrium distribution of the variable $X$ (with $X=\mathbf{v}, \rho, p, \mathbf{B}$ ) and $X_{1}$ its perturbation. The resulting set of linearized equations (see, e.g., [68]) that result after elimination of the terms that reproduce the equilibrium configuration and higher-order terms are

$$
\frac{\partial}{\partial t}\left[\gamma_{0} \rho_{1}+\gamma_{1} \rho_{0}\right]+\nabla \cdot\left[\gamma_{0} \rho_{1} \mathbf{v}+\gamma_{0} \rho_{0} \mathbf{v}_{1}+\gamma_{1} \rho_{0} \mathbf{v}\right]=0,
$$

for the continuity equation,

$$
\begin{array}{r}
\frac{\partial}{\partial t}\left[\gamma_{0}^{2}(\rho h)_{1}-P_{1}+\right. \\
\left.2 \gamma_{0}^{4}\left(\mathbf{v} \cdot \mathbf{v}_{1}\right)(\rho h)_{0}\right]+\nabla \cdot\left[\gamma_{0}^{2}(\rho h)_{1} \mathbf{v}+2 \gamma_{0}^{4}\left(\mathbf{v} \cdot \mathbf{v}_{1}\right)(\rho h)_{0} \mathbf{v}+\gamma_{0}^{2}(\rho h)_{0} \mathbf{v}_{1}\right] \\
+\frac{\partial}{\partial t}\left[B_{0}^{2}\left(\mathbf{v} \cdot \mathbf{v}_{1}\right)+\left(1+v^{2}\right) \mathbf{B}_{0} \cdot \mathbf{B}_{1}-\left(\mathbf{v} \cdot \mathbf{B}_{1}+\mathbf{v}_{1} \cdot \mathbf{B}_{0}\right) \mathbf{v} \cdot \mathbf{B}_{0} / c\right] \\
+\nabla \cdot\left[2\left(\mathbf{B}_{0} \cdot \mathbf{B}_{1}\right) \mathbf{v}+B_{0}^{2} \mathbf{v}_{1}-\left(\mathbf{v} \cdot \mathbf{B}_{0}\right) \mathbf{B}_{1}-\left(\mathbf{v} \cdot \mathbf{B}_{1}\right) \mathbf{B}_{0}-\left(\mathbf{v}_{1} \cdot \mathbf{B}_{0}\right) \mathbf{B}_{0}\right]=0
\end{array}
$$

for the energy, and

$$
\gamma_{0}^{2}(\rho h)_{0}\left[\frac{\partial \mathbf{v}_{1}}{\partial t}+(\mathbf{v} \nabla) \mathbf{v}_{1}\right]+\nabla P_{1}+\frac{\mathbf{v}}{c^{2}} \frac{\partial P_{1}}{\partial t}-\left(\mathbf{j}_{0} \times \mathbf{B}_{1}\right)-\left(\mathbf{j}_{1} \times \mathbf{B}_{0}\right)=0,
$$

for the momentum. The linearized Maxwell equations are

$$
\begin{array}{r}
\nabla \cdot \mathbf{B}_{1}=0 \\
\nabla \times \mathbf{E}_{1}=-\frac{\partial \mathbf{B}_{1}}{\partial t},
\end{array}
$$

with $\mathbf{E}_{1}=-\mathbf{v}_{0} \times \mathbf{B}_{1}-\mathbf{v}_{1} \times \mathbf{B}_{0}$.

Before solving these equations, different approximations/simplifications can be made (and have been made by different authors) in order to study jet stability. The relevance of this selection lies on the fact that different types of instability arise from the different terms, which leads to a selected study of a given instability whereas those embedded in the neglected terms are obviated. According to [69], where the non-relativistic regime was studied, the different terms arising from the linearized equations, 
in the cold limit, lead to the complete list of instabilities that can arise in jetted flows, namely the KHI, CDI, centrifugal buoyancy (Parker instability) and magnetorotational instability (MRI, see also [70]). ${ }^{2}$ The instabilities that can arise in relativistic outflows have a range of causes:

- $\mathrm{KHI}$ arises due to transition layers (either contact discontinuities or continuous changes) in the velocity component tangential to this layer;

- Rayleigh-Taylor instability (RTI) can grow in expanding/contracting jets, with the equivalent to the gravity acceleration being given by the changes in the radial velocity;

- centrifugal instability (CFI) also grows in expanding jets with non-zero azimuthal velocities;

- $\quad \mathrm{CDI}$ arises due to deformations in the toroidal component of the magnetic field, and the so-called pressure-driven instability arises when the magnetic rings that constitute the magnetic structure in the case of toroidal component dominated field are displaced from their equilibrium configuration (under certain magnetic field radial distributions, [71]);

- centrifugal buoyancy is caused by rotation, when the centrifugal force is large enough to bend the magnetic lines;

- $\quad$ MRI can develop in a magnetized, rotating jet with differential rotation (and decreasing angular velocity with radius; see [69]).

The growth of these instabilities compared to the nonlinear regime could imply strong dissipation of kinetic energy, and also magnetic energy via reconnection of magnetic lines (thus departing from ideal MHD).

In the following list, I summarize the different approximations that have been studied in the case of relativistic flows, adding some of the relevant references in which the priority is given to those where the focus is on the linear regime: ${ }^{3}$

Non-magnetized jets $(\mathbf{B}=0)$ :

- $\quad$ No rotation $\left(\mathbf{v}=v^{z} \mathbf{e}_{z}\right)$ :

- No expansion $\left(\partial v^{r} / \partial t=0\right)$ : KHI (e.g., [72-79]).

- Expansion $\left(\partial v^{r} / \partial t \neq 0\right)$ : RTI (e.g., [80-82]).

- $\quad$ Rotation $\left(\mathbf{v}=v^{\phi} \mathbf{e}_{\phi}+v^{z} \mathbf{e}_{z}\right)$ :

- No expansion $\left(\partial v^{r} / \partial t=0\right)$ : KHI (e.g., [83,84]).

- Expansion ( $\left.\partial v^{r} / \partial t \neq 0\right)$ : CFI (e.g., [85]).

Magnetized jets (all non-expanding cases):

- No rotation $\left(\mathbf{v}=v^{z} \mathbf{e}_{z}\right)$ :

- KHI (e.g., [68,86,87]).

- CDI + KHI (e.g., [88-91]).

- CDI/pressure-driven instability (e.g., [71,92,93]).

- $\quad \operatorname{Rotation}\left(\mathbf{v}=v^{\phi} \mathbf{e}_{\phi}+v^{z} \mathbf{e}_{z}\right)$ :

- Centrifugal buoyancy ([94]).

An initial equilibrium state, consistent with the assumptions made, has to be considered prior to solving the equations. The equilibrium state requires constant gas pressure alone if the magnetic

2 The short wavelength pressure-driven (or Z-pinch) instability, which can grow for a particular initial distribution of magnetic field and gas pressure [71] can be taken as a particular type of CDI.

3 There are aspects relevant to the parameter space covered by each of the referred papers (e.g., magnetically versus particle dominated, or hot versus cold jets, etc.) that are not taken into account in this list. 
fields and azimuthal velocity are neglected. The inclusion of the latter terms introduces complexity in the initial radial configuration of the variables due to centrifugal motion in the case of azimuthal velocity and also magnetic tension in the case of the field. These have to be compensated by specific distributions of gas pressure that stabilize the radial structure of the jet, which requires solving the transversal equilibrium equation for magnetized, relativistic jets (see, e.g., [95-98]). In the case in which expansion is considered, equilibrium is applied only at the contact discontinuity (e.g., [80,81]).

The solutions for the perturbations of the variables are taken to have a wave-like form $X_{1}(r, \phi, z, t)=X_{1}(r) \exp \left(i\left(\omega t \pm m \phi-k_{z} z\right)\right)$, where the plus or minus sign that appear with the azimuthal wave number indicate the sense of the wave in the azimuthal direction. The different types of solutions are the result of the combination of the azimuthal and radial structure of the modes:

- If $m=0$, there is no azimuthal dependence and the instability produces axisymmetric oscillations of the jets; $m=1$ produces antisymmetric distributions of the physical parameters that result in helical oscillations; $m=2$ produces elliptical deformations, etc.

- A radial structure with no zeros between the jet axis and its surface (i.e., global changes that do not cross the equilibrium value $X_{0}$ at any point) corresponds to the surface or fundamental modes, whereas if the radial structure crosses the equilibrium value, the modes are called body or reflection modes, with the number of zeros giving the order (one zero for the first body/reflection mode, and so on).

Once the equilibrium is defined and thus the radial profiles of the variables are established, the system can be solved either as a dispersion relation given by matching conditions at a discontinuity between the jet and its environment, or, if the transition is continuous, solving the resulting differential equations by setting boundary conditions on the axis and at $r \rightarrow \infty^{4}$.

In the case of sheared jets, when considering a purely hydrodynamical jet, a single second-order differential equation can be derived for the pressure perturbation (e.g., [77,99-101]). However, when a toroidal component of the magnetic field is considered, the system can only be reduced down to a couple of differential equations, typically taken for the radial velocity and the total pressure $\left(p^{*}\right)$ perturbations. In both cases, the shooting method plus a complex-root finder (e.g, the Müller method, [101]) are used. The derivation of these systems of equations can be lengthy and it is out of the scope of this review. We refer the interested reader to, e.g., [88,89] for explicit derivations.

The solutions of the stability problem are usually given either taking real wavenumbers and complex frequencies (temporal approach), or complex wavenumbers and real frequencies (spatial approach). On the one hand, in the temporal approach, the instability is taken to grow in time at a given location; the imaginary part of the frequency $\left(\omega_{i}\right)$ represents the inverse of the time needed by the instability to e-fold its amplitude and it is called growth-rate. On the other hand, in the spatial approach, the imaginary part of the wavenumber $\left(k_{i}\right)$ represents the inverse of the distance it takes for the instability to e-fold in amplitude. The inverse of $k_{i}$ is named growth-length or growth-distance. In the rest of this paper, I will typically refer to growth-rates, but the reader has to keep in mind that there is an inverse relation between this quantity and growth-lengths, so that an increase of the growth-rate would imply a reduction of the length required to multiply the instability amplitude by a factor $e$. Although this relation is only qualitative and the quantitative conversion from the temporal to the spatial approach requires paying close attention to the group velocity $\left(\partial \omega_{R} / \partial k,[102]\right)$, the qualitative relation is sufficient for the purposes of this paper.

Independently of the type of instability that develops and the jet initial properties, and of the fact that the jet is disrupted or not, the instabilities dissipate kinetic or magnetic energy and can thus act as efficient particle-acceleration mechanisms (e.g., [103,104]).

4 Although there are other optionsi Istomin and Pariev (1994, 1996) [92] consider variations of the field up to the jet radius and set the boundary condition at that point 


\subsection{The Linear Regime}

Out of all the aforementioned papers, several clear conclusions can be extracted regarding the linear growth of jet instabilities. In this subsection, I summarize these ideas.

\subsubsection{Hydrodynamical Jets}

Relativistic hydrodynamic ( $\mathbf{B} \rightarrow 0$ ) jets are $\mathrm{KH}$ unstable because they are typically supersonic and present a velocity transition (either if it is modelled as a contact discontinuity or as a shear-layer) with the ambient medium (e.g., [72-75,77,86,87]).

The growth rates of the KHI modes depend basically on the jet Lorentz factor, internal energy density, transversal structure (sheared jets), and jet expansion (see, e.g., Hardee [78,102,105,106], and references therein; Perucho et al. [79,100,101]). Typically, slower (smaller Lorentz factor) and hotter (larger internal energy density) jets show larger values of the growth-rates [79,100]. In addition, the introduction of a shear layer contributes to a reduction of the growth rates of modes with small wavelengths (comparable to or smaller than the width of the layer), but for the case of faster jets, in which fast-growing, short-wavelength resonant modes appear [99-101]. Interestingly, the resonant modes reported in [101] grow fast enough in the shear region to generate a wide, hot shear-layer around the jet spine, which remains basically untouched (see Figure 1). In the next section, I will discuss the implications of this and other post-linear effects of unstable modes in relation to long-term jet stability. Furthermore, jet expansion favours an increase of the mode wavelength with distance and this typically implies a reduction of the KHI growth-rates [102,107].
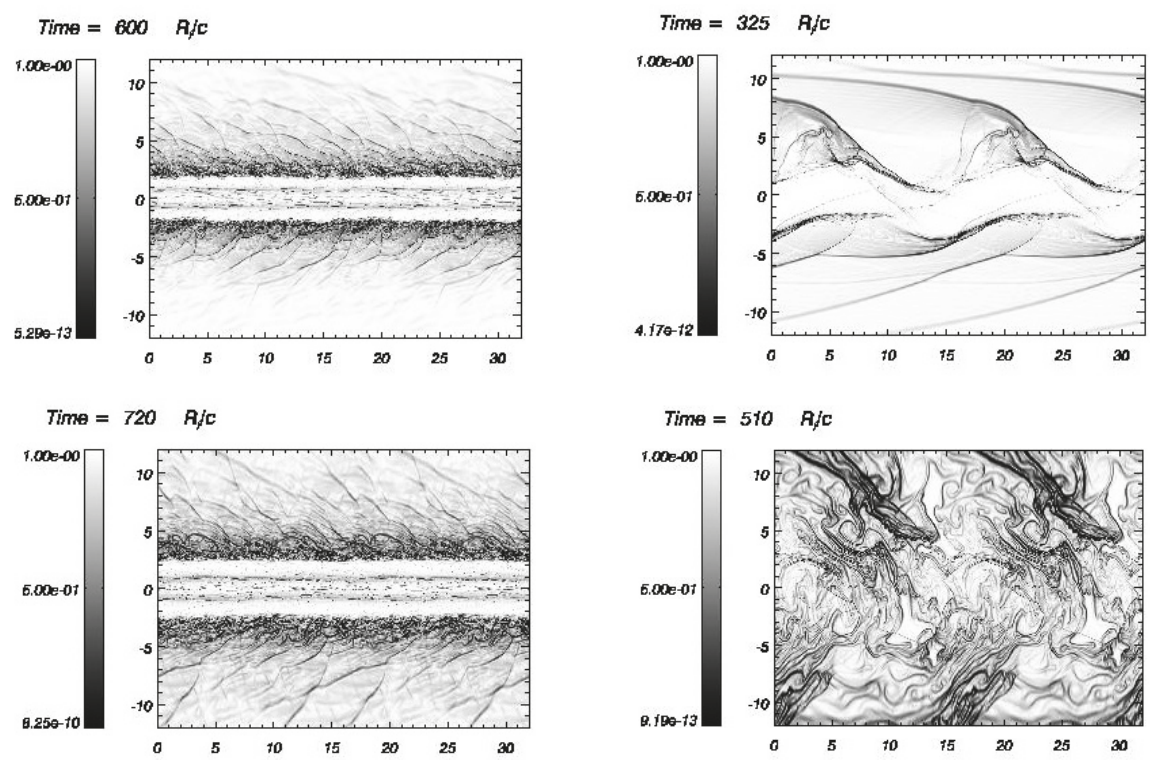

Figure 1. Schlieren plots (enhanced rest-mass density gradients showing waves and shocks) from numerical simulations of two jets at different times [101]. The left column shows the evolution of a jet (fast and relatively cold) in which resonant modes develop at the shear-layer; as a result of the strong dissipation caused by these modes, a thick, hot layer develops surrounding the collimated flow that preserves basically unchanged properties [100]. The right column shows the evolution of a slower jet, in which resonant modes do not dominate the growth rates at the linear regime; in this case, the jet is disrupted by the long-wavelength helical mode. 
The Lorentz factor plays a dual role: in addition to smaller growth rates, the effective distances over which the modes grow are also longer for higher Lorentz factors $(\geq 10)[79,100]$. This is the first important stabilizing factor in relativistic jets when compared to sub-relativistic ones. In contrast, low Lorentz factor jets present higher growth-rates and are thus more prone to the full development of the instability to the nonlinear regime, mass-load and deceleration within relatively short length-scales.

The requirement of small-scale instabilities that can explain the way in which FRI jets decelerate along the first kiloparsecs [27] has brought the attention of theorists to the possibility that the RTI or the CFI develop in expanding jets. Different authors have studied the development of Rayleigh-Taylor instability in jets undergoing expansion and recollimation ([80-82], see Figure 2). In particular, in reference [81], the authors developed the linear analysis and confirmed the prediction given by [80] about the stability condition

$$
\frac{\rho_{1} h_{1}^{\prime} \gamma_{1}^{2}}{\rho_{2} h_{2}^{\prime} \gamma_{2}^{2}}>1
$$

where subscripts 1 and 2 indicate the jet flow and the cocoon, respectively, $\rho$ is the rest-mass density, $\gamma$ is the Lorentz factor and $h^{\prime}$ is defined as

$$
h^{\prime}:=1+\frac{\Gamma^{2}}{\Gamma-1} \frac{p}{\rho}
$$

with $\Gamma$ the adiabatic exponent of the fluids, assumed to be constant across the contact discontinuity. Equation (14) states that the system is unstable when the inertia of the jet is larger than that of the cocoon or surrounding medium. The authors concluded that this instability is thus mainly excited in oscillating jets with high inertia, as opposed to KHI, where growth-rates are reduced by the jet inertia. In [82], the authors have shown that the development of the RTI instability (or Richtmeyer-Meshkov instability in the case of impulsive expansion) can be extended to different spine-sheath configurations. This work shows that the spine does not necessarily need to be 'heavier' than the cocoon for the RTI to develop, because the acceleration vector is inverted in the reconfinement region with respect to the expansion region and the roles of the jet and cocoon are thus interchanged, also producing small-scale mixing at the jet boundary.

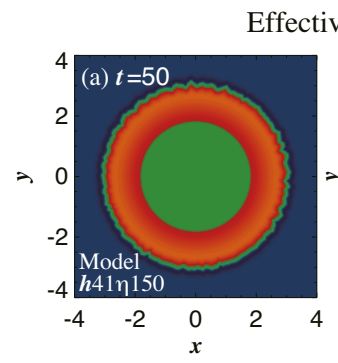

Effective inertia
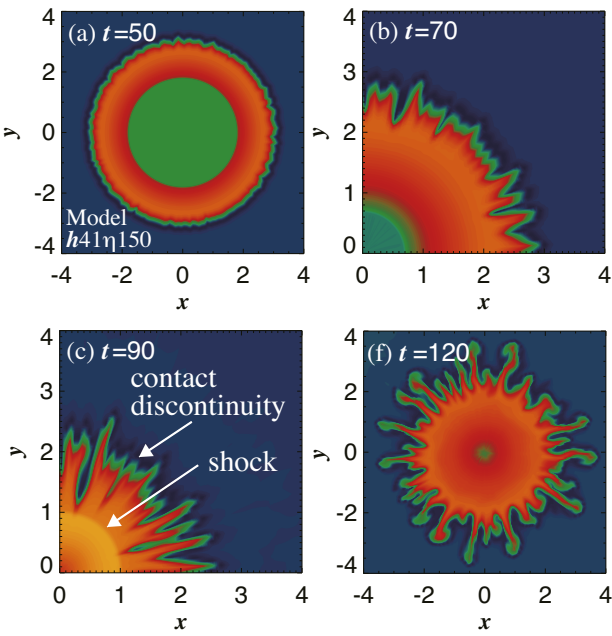

$$
\begin{gathered}
\log \gamma^{2} \rho \boldsymbol{h} \\
2.5 \\
2.0 \\
1.5 \\
1.0 \\
0.5 \\
0.0 \\
-0.5
\end{gathered}
$$

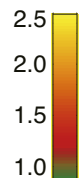

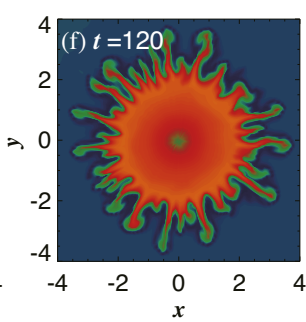

Figure 2. The image shows panels of the logarithm of the effective inertia $\left(\gamma^{2} \rho h\right.$ in units of ambient rest-mass energy density, $\rho_{a} c^{2}$ ) along an expanding and recollimating jet from the simulations in [80]. The development of the RTI can be clearly observed (adapted from [80], courtesy of Jin Matsumoto). 
Meliani and Keppens $[83,84]$ studied the development of instabilities in the shear between rotating cylinders, and interpreted them as RTI developing due to centrifugal acceleration. Gourgouliatos and Komissarov [85] showed that this is actually the relativistic version of the CFI and that it may grow in flows with curved streamlines such as jets with oscillatory cross-sections caused by expansion and recollimation (see Figure 3). The authors generalize the Rayleigh criterion to the relativistic case for a discontinuity separating two rotating flows to

$$
\Psi_{2}-\Psi_{1}<0,
$$

with

$$
\Psi=\rho h \gamma^{2}\left(\Omega R^{2}\right)^{2}
$$

where $\Omega$ is the angular velocity, for a ring of fluid pushed from medium 1 to medium 2 (which can be identified with the jet and the cocoon, as above, in the expansion phase). The authors point out that when $\Omega$ is constant across the discontinuity, this condition results in

$$
\rho_{2} h_{2} \gamma_{2}^{2}-\rho_{1} h_{1} \gamma_{1}^{2}<0
$$

or $\rho_{2}-\rho_{1}<0$ in the non-relativistic case, which is the RTI criterion given in Equation (14).

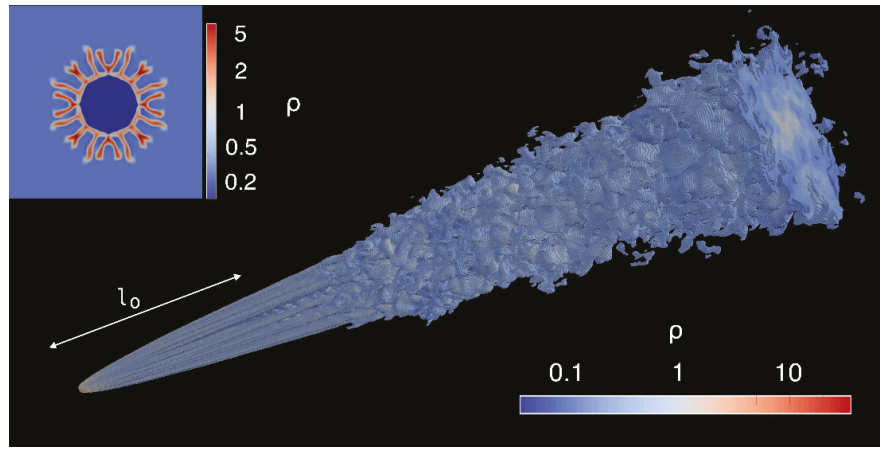

Figure 3. Rest-mass density contour (in arbitrary units) from a simulation in which the development of RTI produces the decollimation and deceleration of a relativistic jet [108] (courtesy of Konstantinos Gourgouliatos).

In [85], the authors also derive a criterion for the case of a continuous transition between the two flows (shear). In this case, they derive

$$
\frac{\mathrm{d} \ln \Psi}{\mathrm{d} \ln R}<M^{2}
$$

with $M=\gamma \Omega R /\left(\gamma_{s} \mathcal{c}_{S}\right)$, and $\gamma_{s}$ the Lorentz factor of the sound speed. This criterion is; however, still to be tested by numerical simulations.

\subsubsection{Magnetized Jets}

\section{KHI and CDI}

The study of the stability of relativistic jets threaded by magnetic fields started from the relatively easier configurations of purely axial magnetic field (e.g., [68,91]). In reference [68], P. Hardee studied 
the stability of magnetized jets with a spine-sheath structure ${ }^{5}$ and showed that this configuration results in the stabilization of the flow with respect to 'naked' jets, to the extent that trans-Alfvénic and super-Alfvénic spines can become stable to the KH instability (unlike in the relativistic hydrodynamics, RHD, case, where transonic spine-sheath configurations are still unstable), but for the fundamental pinch mode. The development of the KH instability in planar flows with equal velocity modulus and opposite sign, threaded by a uniform magnetic field parallel to the velocity direction was studied in [91], where the conclusion that stronger magnetic fields and flow velocities stabilize this configuration was also reached.

Bodo and collaborators [88] tackled the stability of relativistic, zero pressure (cold), magnetized jets with different rotation and helical field configurations leading to different current distributions within the jet, and return currents located outside the shear layer. They solved the linearized system of equations by means of the shooting method with a complex secant root finder, setting appropriate boundary conditions close to the jet axis and at radius $r \gg R_{j}$. A critical point that is stressed in these works is the role played by the so-called resonant surfaces, where $\mathbf{k} \cdot \mathbf{B}=0$ : the absence of the magnetic tension in the plane perpendicular to the field line facilitates the development of the CDI in that direction. Expressing this scalar product in terms of the pitch parameter (defined as $\left.P=r B^{z} / B^{\phi}\right)$, ${ }^{6}$ they obtain (see [88])

$$
\mathbf{k} \cdot \mathbf{B}=k_{z} B^{z}+\frac{m}{r} B^{\phi}=0, \quad \text { or } \quad k_{z} P+m=0
$$

Interestingly, the wavenumbers larger than $m / P_{c}\left(P_{c}\right.$ is the value of $P$ at the axis, which converts to $\gamma P_{c}$ in the bulk plasma reference frame) are stabilized, and only those values that are smaller show unstable solutions (see Figure 4) for the magnetic field distributions considered. As a consequence, the authors find that a purely longitudinal field is only $\mathrm{KH}$ unstable (the KHI growth rates do not depend strongly on the pitch parameter).

Another relevant parameter is the ratio of total gas to magnetic energy densities, $M_{a}$ (which in the case of cold jets, as considered by Bodo and collaborators, reduces to the kinetic to magnetic energy density ratio): for large values of this parameter, i.e., matter dominated flows, the jets are only $\mathrm{KH}$ unstable; the jet is stable with respect to KHI below a certain value of $M_{a}$ that depends on the pitch parameter, with this threshold moving to larger values of $M_{a}$ for larger values of $P_{c}$ (i.e., smaller toroidal fields). For dominating toroidal fields, this threshold moves to smaller values of $M_{a}$ and the KHI and CDI curves merge in the solution plane (see Figure 4). As a global conclusion, one can state that a strong poloidal field stabilizes the jets, unless $M_{a}$ is large, i.e., unless the plasma is highly super-Alfvénic [88]. Irrespectively of the poloidal field intensity, the jet only becomes $\mathrm{KH}$ unstable in the case it dominates the field structure. We have to note, nevertheless, that a strong poloidal field would set demanding limits on the values of the field at injection $\left(B_{z} \propto R_{j}^{-2}\right)$.

5 Despite this structure is arbitrarilly introduced in the set-up of many simulations and stability analysis, a sheath is probably surrounding the fast jet spines, either from the jet formation region, where a wind can be formed from the accretion disk [109], or because of simple kinetic energy dissipation at the jet-environment shearing layer.

6 Please note that the definition of the pitch varies among papers and that it can also be found as $\psi(r)=\arctan \left(B^{\phi}(r) / B^{z}(r)\right)$. 

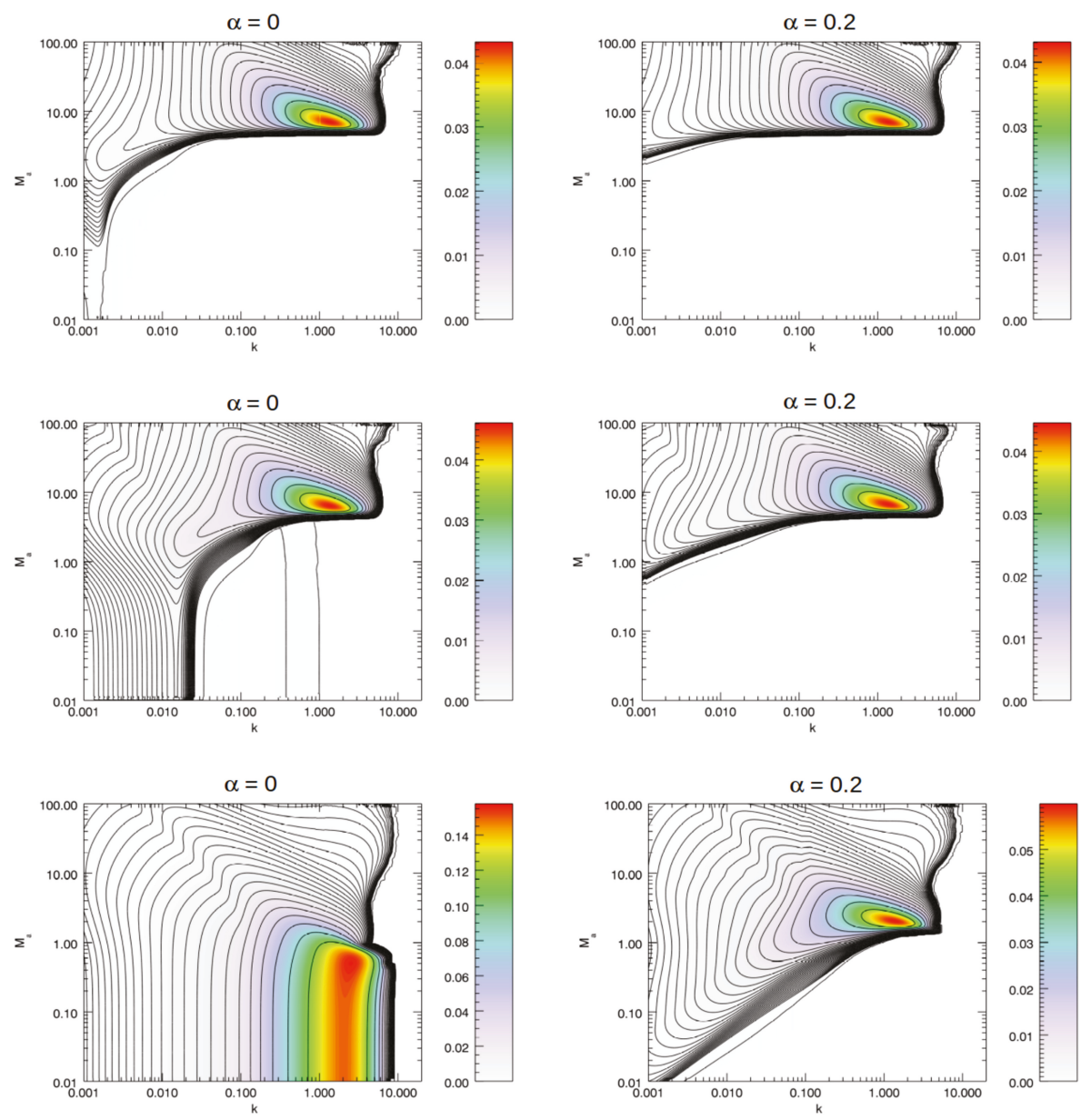

Figure 4. Growth-rate contours of instabilities in a zero pressure, magnetized, relativistic jet as a function of the wavenumber, $k$, and the ratio of magnetic to kinetic energies, $M_{a}$, for a series of initial jet structures [94]. In all the plots, the jets have Lorentz factor 10. The left column shows models with no rotation, whereas the right column shows models with rotation (the parameter $\alpha$ gives a measure of rotation speed, see [94]). The upper panels give the solutions for $P_{c}=10$, i.e., with a dominating poloidal field (see the text), the middle panels give the solutions for $P_{c}=1$, and the bottom panels for $P_{c}=0.1$, i.e., dominating toroidal field. The region with $M_{a}>1$ displays the KHI solutions, and the CDI appears at low values of this parameter, i.e., for magnetically dominated jets, and mainly when the toroidal component of the field is more important (courtesy of Gianluigi Bodo).

Unlike for the KHI, the CDI growth rates are basically independent of $M_{a}$, and increase with $B^{\phi}$. The resonant condition $(\mathbf{k} \cdot \mathbf{B}=0)$ makes the CDI growth rates change according to the value of the pitch parameter, which can vary across the jet, for instance, when the field distribution results in a return current at the shear layer [88]. This situation results in a non-straightforward relation between the growth rates of instabilities with different wavelengths at different radii, with the modes being stabilized where the pitch parameter changes fast, i.e., in the region where the current is located, but the modes can still grow inside and outside of this region. This is easily explained using Equation (20): if 
$B^{\phi} \rightarrow 0$ when $r \rightarrow 0$, the resonance can only be obtained for small wavenumbers, whereas the opposite is true for large values of $B^{\phi}$ (when $r \rightarrow R_{j}$ ).

Kim et al. $[89,90]$ have studied the stability properties of jets without current-sheets at the jet boundary. The magnetic field distribution that these authors use is based on a tractable equilibrium solution for a current-sheet free jet, extracted from the set of solutions derived in [96], with both the axial and toroidal components becoming zero at the jet boundary. The distributions also imply zero net poloidal current along the jet cross section. Their approach allows for non-zero pressure plasmas, making it more general. The authors discuss the stability properties of such jets in the linear regime, confirming the stabilizing role of the Lorentz factor, in terms of decreased growth rates at all wavelengths, in agreement with the RHD case [79,100]. With the proposed magnetic field configuration, increased magnetization stabilizes the pinching fundamental mode and the helical (or kink) fundamental mode at short wavelengths. However, it does not affect the growth rates at long wavelengths for the helical mode, leaving room for the growth of these disruptive modes. An extension of their work to velocity-sheared jets showed that the presence of a shear layer causes a drop in the growth-rates of the short-wavelength modes (as it is also the case in hydrodynamical jets), but again it does not show a strong effect on the long-wavelength, fundamental kink modes [90]. In [110], the authors claim that the magnetic field configuration given by Bodo et al. [88] implies a constant value of the pitch parameter across the jet, which makes the CDI to become relevant according to Equation (20) (see also Figure 4), and also to be suppressed from a given wavenumber $\left(k_{z} \sim 1 / P\right)$. In contrast, if the pitch parameter changes across the jet, such a limit in the development of CDI at high wavenumbers disappears, and the CDI modes show up at all wavenumbers, albeit less enhanced than in the distributions used in $[69,88,94]$.

Numerical simulations by Mizuno and collaborators for static columns [111] have shown that $\mathrm{CDI}$ is stabilized by a dominating axial field and that the pitch angle profile can also change the growth of the unstable modes: the growth rates of the kink mode are reduced by an increase of the pitch parameter (defined as in [88]) with the radius (i.e., if the toroidal field becomes relatively weaker), and they increase if the pitch parameter falls with the radius. In [112,113], the authors found that the location of the velocity shear in a sub-Alfvénic jet can also determine the growth rates of CDI modes and whether kinks are very slowly propagating or move downstream at faster speeds, with larger velocity shear radii (with respect to location of the peak value of the toroidal field) reducing the mode growth rates and also making the kinks slower.

The possible development of the pressure-driven or 'Z-pinch' instability in jets was also pointed out in [71] (see also, e.g., [114], for rotating MHD jets, and [115]) with pressure and magnetic gradients across curved magnetic lines as those in toroidal/helical configurations. The pressure gradient arises naturally to generate equilibrium configurations when magnetic pressure and tension have to be compensated (see, e.g., [97] and references therein). In such a situation, the displacement of a magnetic loop can trigger the growth of the instability if the toroidal field increases with radius, consistently with the conclusions derived for the CDI (see above). This instability can destroy the concentric structure of the magnetic field close to the jet spine, endangering the possible collimating role of the magnetic field. Maximum growth-rates correspond to small axial wavenumbers in this instability, so no global (large-scale) effects would be visible in the jet structure, other than the probable radiative outcome of dissipation. Nevertheless, it was pointed out [115] that a velocity gradient (in the region where the instability develops) can be a compensating mechanism against the pressure-driven instability.

\section{Stability of magnetized, rotating jets}

The introduction of a rotation velocity in the flow represents a stabilization effect for CDI, mainly for more magnetized jets [94], but does not strongly affect the KH unstable modes. For small values of $P_{c}$ (i.e., dominating toroidal field), the CDI instability appears at smaller wavenumbers (in other 
words, larger wavenumber are stabilized) with decreasing $M_{a}$, which means that rotating, strongly magnetized jets are more stable against the CDI (see also $[92,93,95,116]) .^{7}$

In the case of rotating flows with helical fields, an equivalent to Parker instability arises in the jets, where the role of gravity in that case is played by the centrifugal force $($ see $[69,94]$ and references therein). The growth rates of this instability increase with the square of rotation frequency, $\Omega$, and are independent of the wavenumber. The instability shows up at low wavenumbers for the toroidal buoyancy mode and at large wavenumbers for the poloidal one. In [69,94], the authors conclude that the modes are stabilized by large values of $M_{a}$ (i.e., particle dominated) and also in the magnetically dominated limit $M_{a} \rightarrow 0$ (i.e., they only appear at intermediate values of $M_{a} \sim 1-10$ ) for the studied cases. Millas et al. [117] revisited the simulations of rotating cylindrical flows run in [83,84] and showed that the inclusion of a toroidal component stabilized the development of RTI (or CFI) in rotating jets.

\subsection{The Non-Linear Regime (or 'Then, Why Are (Some) Jets So Stable?')}

In the previous section, I have described the instabilities that grow on the configurations that have been studied by different authors, and the conditions under which the unstable modes can have larger or smaller growth-rates. In summary, from the studies performed so far we can state that RMHD jets are more stable when (1) the toroidal component of the field is smaller, (2) the pitch parameter changes within the jet, (3) the jet rotates with relatively small rotation frequencies, (4) in those parts of the jet cross-section where the toroidal field falls with the radius, (5) the jets are surrounded by shear-layers and/or expand, and (6) have more inertia. Table 1 summarizes these general trends for the KHI, CDI and RTI/CFI.

Table 1. Summary of the stabilizing/destabilizing factors for each of the most relevant/studied instabilities in relativistic jets so far. A $\checkmark$ symbol indicates a stabilizing effect and $\times$ indicates a destabilizing effect as the parameter grows. From left to right, the parameters are: jet radius (expansion), jet inertia, poloidal field, toroidal field, shear-layer width, and toroidal velocity. The - sign indicates that there is no effect or that it is ambiguous, depending on factors like radial distribution. The table only pretends to give general and orientative trends but different radial distributions or combinations can change the trends given (see the text).

\begin{tabular}{lcccccc}
\hline & $\boldsymbol{R}_{j}$ & $\rho_{j} \gamma_{j}$ & $\boldsymbol{B}^{z}$ & $\boldsymbol{B}^{\phi}$ & $\delta_{s}$ & $v^{\phi}$ \\
\hline KHI & $\checkmark$ & $\checkmark$ & $\checkmark$ & - & $\checkmark$ & $\checkmark$ \\
CDI & $\checkmark$ & $\checkmark$ & $\checkmark$ & $\times$ & $\checkmark$ & $\checkmark$ \\
RTI/CFI & $\times$ & $\times$ & - & $\checkmark$ & - & $\times$ \\
\hline
\end{tabular}

However, from the description given, one can extract the (correct) impression that there is no completely stable configuration and that jets are prone to destabilization under basically any initial distribution of the physical parameters, the difference being the time/distance in which the unstable modes grow to nonlinear amplitudes. In addition, we conclude that there is something that we do not understand in jet physics because, according to linear theory, all jets should be destroyed by instabilities at some point.

The incautious reader has to be thus warned about the limits of linear theory when applied to extragalactic jets. The linear studies actually face the difficulty of having to impose very specific initial configurations that equilibrate the transversal profiles of jets under several a priori assumptions about the magnetic field distribution across the jet. This situation is forced by the impossibility to get a clear picture of the magnetic field structure in jets from observations, due to relativistic and

7 Istomin \& Pariev $[92,93]$ studied the development of the CDI in a force-free jet. The authors found that the shear in the toroidal field plays a strong stabilizing role both for axisymmetric and non-axisymmetric perturbations, in the absence of gas pressure, by prohibiting radial convective motions. 
projection effects $[118,119]$. In addition, linear analysis is usually based on the consideration of infinite jets. We have seen, within this assumption that even if an unstable mode has a large growth rate under certain jet parameter configuration, this may rapidly change under changing conditions (see the previous section), which might be the case either across an inhomogeneous jet (for short wavelength modes), or along a jet with changing properties in distance (e.g., due to expansion, mild entrainment ..., see Section 3).

Moreover, there is possibly a generalized misconception caused by the extension of growth rates in the linear regime to their destabilizing role: we tend to think that the most disruptive instability modes are those showing larger growth rates and that the most unstable jets (and thus more prone to disruption) are those for which the growth rates are larger. However, the link between the linear and the non-linear regime was shown not to be straightforward by numerical simulations $[39,100]$, and even fast growing modes may be non-disruptive (e.g., see Figure 1, [101], and see also [120] for an application to the radio galaxy $3 C$ 111). Such fast-growing, non-disruptive modes are necessarily short wavelength instabilities that induce small scale changes in the flow shear-layer. As a result, the jet structure and properties (velocity, composition, magnetization, etc.) may change due to dissipation and minor entrainment at the jet boundary, but the outcome would still be considered a collimated, long-term-stable jet.

In this respect, the pressure-driven instability [71] could disrupt the toroidal structure of the flow, possibly dissipating magnetic and kinetic energy, but if the jet retains (at least to some extent) the axial field structure, this component can reduce the growth rates of the KHI and completely stabilize both the CDI. ${ }^{8}$ However, it is implausible that the poloidal field dominates the jet dynamics at large scales on the basis of magnetic flux conservation (see above).

Not all short wavelength instabilities are harmless to jet collimation. In fact, small-scale RTI or CFI modes have been proposed as the cause of the progressive deceleration in FRI jets [81,108]. In this case, the difference lies on the jet properties: both the RTI and CFI require jet expansion with relatively large opening angles, i.e., slower, overpressured (hot) jets propagating in dilute media or through steep pressure gradients.

Longer wavelength instabilities do develop in jets and we can explain the structures forced by these growing large-scale perturbations, considering global jet properties and the small jet opening angles (actually, jets can freely expansion and still have a small opening angle, $\sim 1 / \gamma$, to fulfill the linear theory basic assumptions). This idea was successfully applied to several well-known radio galaxies and quasar jets (e.g., [106,124-129]), typically for cases in which the observed wavelengths are long enough, $\lambda \gg R_{j}$, not to depend critically on the jet internal fine structure. It was also shown, by means of numerical simulations, that the instabilities can develop and show up only at given regions of the jet cross-section and be detected only by observations at the frequencies that reveal those regions [130].

Regarding long-term jet stability, I review and enlarge here the list of arguments that can make jets stable for long distances. First of all, I would like to clarify the meaning of 'jet disruption' or 'long-term stability' in order to set the frame of the discussion. From this author's point of view, a jet is disrupted when it is strongly mass-entrained and decelerated, losing collimation and acquiring plumed structure in radio images. Under this point of view, jets can develop instabilities reaching the non-linear regime, and even be entrained and decelerated, without becoming a 'disrupted jet'. In other words, a jet is not disrupted unless it is strongly decelerated and losses the inertia that contributes to its collimation, even if its composition, transversal structure or magnetization change. Therefore, we have to understand 'long-term stability' as the ability of jets to reach hundreds of kiloparsecs without being decollimated. We can think, for instance, about the prototypical jets in Cygnus A and consider them as

8 In fact, FRII jets show polarization structure at kpc scales that is compatible with a dominating axial component [121-123], thought to be produced by shearing. 
long-term stable FRII jets. However, its jets show an obvious large-scale kink close to the hot-spots, so 'stability' has to be taken as a relative term.

A very relevant, and stabilizing, difference of the growth of instabilities in relativistic flows was pointed out by M. Hanasz [131], who predicted that the amplitude of the $\mathrm{KH}$ unstable wave would cease to grow as soon as the amplitude of the velocity perturbation approaches the speed of light in the jet reference frame. This was later confirmed by numerical simulations $[79,100,132]$. The physical limit imposed by the speed of light shows to be efficient in avoiding the indefinite growth in amplitude of the unstable modes, thus providing the flow with a chance to avoid disruption. Nevertheless, the simulations also showed that for a large area of the parameter space (relativistic Mach number vs. Lorentz factor, see [100]) the instabilities reach an amplitude that is enough to disrupt the flow before the limit is reached.

Jet expansion can also represent a stabilizing mechanism [102]: the KHI modes grow when reflected at the discontinuity/shear-layer between the jet and the ambient medium (with the jet acting as a resonant waveguide, [133]), so the increased distance to be covered by the wave to reach that boundary increases the growth-length of the mode. In $[89,90]$, for instance, the authors compare the growth time-scale of the unstable modes $\tau=1 /\left|\omega_{i}\right|$ with the propagation time-scale $T=K R_{j} / v_{g}$, where $K$ is a constant indicating the number of jet radii considered (for an instability to develop to the nonlinear regime), and $v_{g}$ is the group velocity. The comparison between $\tau$ and $T$ gives an idea of the disruptive potential of a given mode (e.g., if $\tau<T$, the growth rate of the mode is too slow to destabilize the flow). Thus, we can also say that expansion increases $\tau$, favoring jet stability. In fact, expansion was even proposed as the main factor to explain jet collimation at large scales, via the loss of causal connectivity across the jet if the ambient pressure, $p_{\text {ext }} \propto z^{-\kappa}$, falls with $\kappa \geq 2$ [107].

Surrounding winds formed from the accretion disk [109] or thick shear layers can contribute to reduce the growth of instabilities, as shown by linear analysis (see Section 2.2), and also numerical simulations (e.g., $[113,134,135])$.

Finally, there is a key point in this discussion: jets gain stability with respect to the KHI, for instance, as they evolve through an ambient medium that becomes more dilute (this also applies to the cocoon, with a decreasing density as it expands, see, e.g., [102,107]). Therefore, those jets with larger head advance speeds can reach large distances before any instability can trigger their disruption, which has to be considered in parallel to the fact that larger bulk Lorentz factors result in smaller instability growth-rates. Furthermore, a recent paper [20] showed that small-amplitude oscillations of the jet head caused by linear helical unstable modes can cause accelerated head velocity down density/pressure gradients across and out of active galaxies (see Figure 5). Therefore, the old Catalan saying qui dia passa, any empeny, with approximate translation 'that who survives a day, pushes a whole year', can be applied to long-term-stable jets. In other words, jets are unstable systems but, in some cases, manage to reach long distances before any growing instability endangers their collimation (e.g., FRII sources), whereas others have enough inertia to develop to large scales (through a decreasing density ambient medium) even if mass-loaded and decelerated, i.e., 'disrupted'. In this case, the jets expand in the turbulent regime, but can still propagate to large distances in external density/pressure gradients ([28], e.g., large-scale FRI sources as the radio jets in 3C 31, which reach $\geq 200 \mathrm{kpc}$ ). 


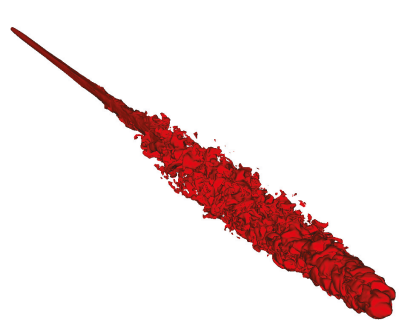

(a)

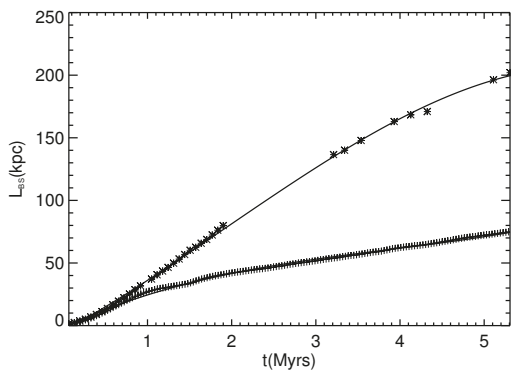

(b)

Figure 5. (a) The left panel shows the jet-mass fraction 50\% isosurface for a 3D RHD simulation of a powerful relativistic jet propagating through a density/pressure gradient typical of an elliptical galaxy [20]. The jet collimation reminds that observed in FRII jets. (b) Jet head position versus time for the 3D simulation shown in the left panel and a 2D axisymmetric simulation with the same injection parameters, for comparison; the lines show polynomial fits to the data. The 3D jet propagates faster due to head acceleration produced by the obliquity of the front shock as compared to the planar shock typically observed in 2D simulations (see [20] for a detailed discussion).

\section{Jet Star/Cloud Interactions}

On top of instabilities, there is another efficient mechanism to convert magnetic or kinetic energy into internal energy and particle acceleration, ${ }^{9}$ which is direct entrainment. It can be caused by the growth of small-scale instabilities causing the formation of turbulent layers that propagate toward the jet center, or by the direct impact of the jet with a stellar wind or a gas cloud. In the latter, the supersonic nature of jets triggers shocks and turbulent mixing tails of shocked jet and wind plasma (see the left panel in Figure 7). This scenario can thus also be a source of particle acceleration via first-order Fermi acceleration in shocks, Fermi II acceleration in the turbulent mixing tails, or shear acceleration (e.g., [49] and references therein).

It was first noted in [60] that the interaction between a star and the jet flow could be responsible for gamma-ray production in extragalactic jets. Before, these interactions had been claimed to be possibly responsible for the knotty structure of M87 [136]. Although this is not the most accepted scenario for M87, the interaction was proposed to explain gaps in radio emission at the sub-parsec scale in Centaurus A [137] and its knottiness at kiloparsec scales [138-140] (see Figure 6), and also as an explanation for the gamma-ray emission $([61-66,141])$ and flares recurrently observed in radio galaxies (M87, [142-147]), or blazars (3C 454.3, [148], or CTA 102, [149]).

Numerical simulations of this scenario, in the context of relativistic flows, have only been performed in the RHD limit [150-152]. These simulations were focused on the dynamical and energetic impact of the interaction, plus the expected radiative output. In [150], the authors studied the evolution of jet/star interaction where the star is surrounded by an envelope or wind before the system is stabilized (see below). The simulations showed that these envelopes can be completely disrupted before the star crosses the jet (see also [153] for relativistic flow-cloud interactions in X-ray binaries), thus incorporating a large amount of hadronic material in the flow. The shock is stabilized at a

9 The direct conversion of magnetic energy into internal energy implies dissipative mechanisms beyond an ideal MHD description. 
distance from the star that is given by the equilibrium between the jet and the stellar wind ram pressures [42,151]:

$$
R_{s}=\sqrt{\frac{\dot{M}_{w} v_{w}}{4 \pi \rho_{j} \gamma_{j}^{2} v_{j}^{2}}},
$$

where $R_{s}$ provides the location of the contact discontinuity, $\dot{M}_{w}$ is the stellar wind mass flux, and $v_{w}$ is the wind velocity, and the subscript $j$ refers to jet values (note that the velocity is in physical units in this expression). During the phase prior to equilibrium, the shocked stellar wind forms a bow-shock structure and a cometary tail along which the shocked wind is accelerated (see Figure 7). If the tail is destabilized, the shocked jet and shocked wind flows can eventually mix. As a result of the jet-star/cloud interactions, the resulting jet transversal structure probably becomes significantly irregular, with slower/colder streams of gas continuously flowing and mixing with the jet flow and thus becoming an excellent location for particle acceleration. The key point is to know in which scales and amounts we expect this to happen. On the one hand, the high mass and inertia of the cometary tail provide it with remarkable stability [150,152], but 3D simulations show that the asymmetric perturbations forced by the propagation of the star through the jet probably induce non-linear oscillations that can already destroy the tail close to the interaction region [152] (see the right panel in Figure 7). Therefore, turbulent mixing is expected to take place not far downstream from the interaction site.

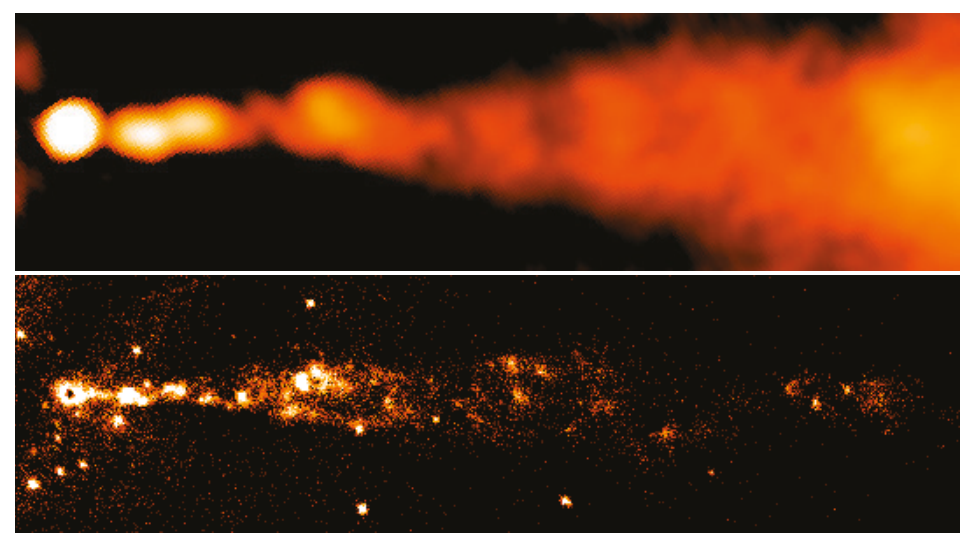

Figure 6. Centaurus A jet as observed by the VLA at $5 \mathrm{GHz}$ and by Chandra at X-rays. Adapted from [138] (courtesy of Martin Hardcastle). The X-ray knottiness of this jet was interpreted as possible jet-massive star interactions. In the radio, the emission shows a diffuse structure.

A very detailed entrainment/deceleration model, based on a previous study of the stellar population in Centaurus A, was developed by Wykes et al. [64], who estimated that there could be $10^{8}-10^{9}$ stars within the jet, entraining a total of $\sim 10^{-3} \mathrm{M}_{\odot} / \mathrm{yr}$, and could reproduce the spectral distribution of the kpc-scale jet from radio to X-rays with this model by means of the synchrotron emission produced by the interactions (via the Fermi I mechanism). The radiative output of the interaction depends on the location along the jet, with close impacts to the SMBH possibly explaining gamma-ray flares via proton-proton interactions, as proposed for M87 [147], but even with collective downstream interactions underpredicting the steady gamma-ray flux detected from this galaxy [65]. Vieyro et al. [65] studied the collective synchrotron plus IC emission caused by jet-star interactions (with the seed IC photons considered to be infrared photons in the case of starburst galaxies and starlight plus CMB for galaxies with older stellar populations, e.g., M87). The authors applied their model to different cases, including the FRI radiogalaxy M87, and concluded that the diffuse radiation 
emitted by these interactions can be strong at X-rays via synchrotron, although the X-ray emission is more structured in the case of M87 (cf. other FRI jets showing diffuse emission [27,46]). Furthermore, the gamma-ray emission produced via IC radiation could significantly contribute to the persistent gamma-ray flux received from the studied galaxies. According to Torres-Albà and Bosch-Ramon [66], the dependence of the radiative output with the jet power is relatively weak in the studied range $\left(10^{43} \mathrm{erg} / \mathrm{s}<L_{j}<10^{45} \mathrm{erg} / \mathrm{s}\right)$, and synchrotron photons up to $100 \mathrm{MeV}$ could be produced for a weak magnetic field (with respect to equipartition) or by bright events added to the persistent background in the case of stronger field intensities. The authors derived total emitted synchrotron luminosities $\gtrsim 10^{40}-10^{42} \mathrm{erg} / \mathrm{s}$ and inverse Compton $\sim 10^{39}-10^{40} \mathrm{erg} / \mathrm{s}$ as a result of the collective interaction of jets with a red-giant population. The role of Doppler boosting on the received flux was also highlighted in these works (see [141] for the case of blazars), with the obvious result that misaligned sources are more difficult to detect. A more exotic scenario is the interaction of the jet with a supernova explosion [154], which could result in stronger gamma-ray emission (IC of infrared photons or synchrotron self-Compton producing up to $10 \%$ of the jet power for jets in the range $10^{43}-10^{44} \mathrm{erg} / \mathrm{s}$ ) collimated along the direction of the flow by Doppler boosting, if the jet-supernova interaction occurs at tens of parsecs (the region where a larger number of explosions could be expected) and the ejecta is accelerated downstream of the jet to a high Lorentz factor. This contribution could be steady in the case of galaxies with high star formation rates and weak blazar jets. The model considers that the shocked supernova remnant does not mix with the flow along a sufficient distance, so it obviates the jet deceleration, which according to the authors, would eventually occur farther downstream from the emitting region $(\sim \mathrm{kpc})$.

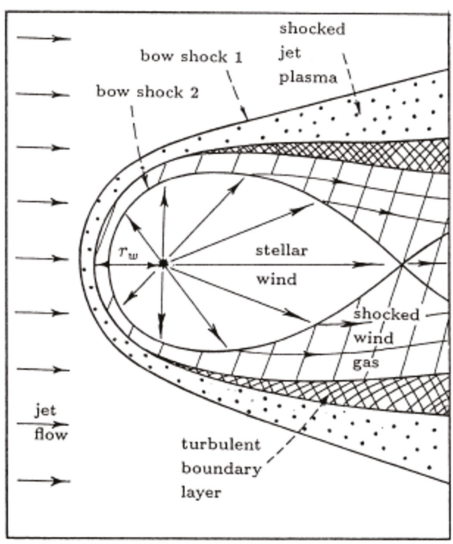

(a)

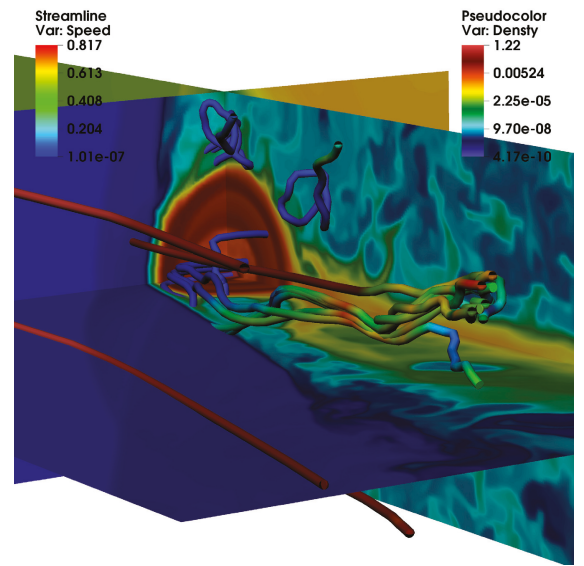

(b)

Figure 7. (a) Schematic plot of jet-stellar wind interaction [42]. (b) Rest-mass density distribution from a 3D RHD simulation of a star/wind bubble system entering into a jet [152]. The image shows three cuts across the grid, displaying the position of the wind injector (star). The color scales indicate the speed (top left) and the rest-mass density (top right). The star enters the jet from the right to the left, which is occupied by the jet flow. The jet flow appears in blue color, with a velocity directed towards the bottom of the image. The current lines show, on the one hand, the deviation and deceleration of the jet flow as it crosses the bow-shock, and, on the other, the shocked wind material being dragged downstream and forming an unstable tail.

Figure 8 shows different spectral energy distributions for a jet-red giant interaction at the equilibrium stage (i.e., once all the outer wind layers have been eroded and the interaction is located at $R_{S}$ ) as observed from different viewing angles (left panel) and at different locations along the jet 
(right panel, [151]). The radiative output is modest, but it has to be kept in mind that it is the collective emission of all jet-star interactions that can be observed by our detectors. Altogether, the different works coincide on the efficient local conversion of kinetic energy into internal energy at the interaction region between the jet and red giants or massive stars and the plausibility of $\mathrm{GeV}-\mathrm{TeV}$ radiation generated from these regions. This scenario thus represents a possible explanation, along with (i.e., not excluding) the spine-sheath [155] or the jet-in-jet [156] models, for VHE emission from AGN jets. Finally, the interaction scenario was also recently proposed as a plausible option to explain the production of neutrinos in blazars via the hadronic processes following the entrainment of protons from stellar winds [157-161].

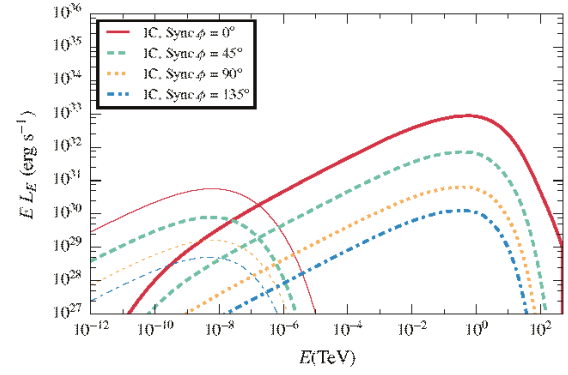

(a)

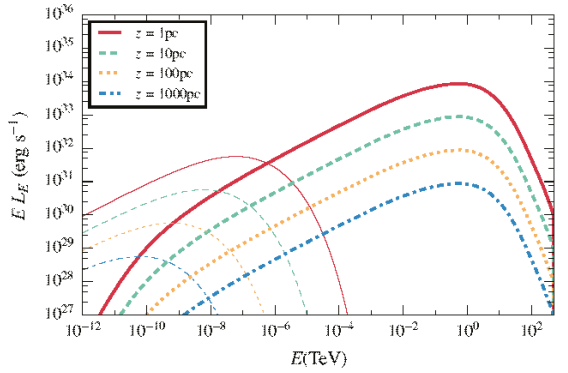

(b)

Figure 8. (a) Resulting spectral energy distributions (SED), including synchrotron and IC radiation, of the equilibrium state in a jet-star (red giant, $\dot{M}_{w}=10^{-9} \mathrm{M}_{\odot} / \mathrm{yr}$ ) interaction at $10 \mathrm{pc}$ from the central engine for a weakly magnetized jet, as derived from different viewing angles [151]. An increase of the magnetization increases strongly the synchrotron flux but barely affects the IC radiation. (b) Same as (a), but for a fixed viewing angle of $\phi=0^{\circ}$ and different interaction locations along the jet. The farther from the central engine, the weaker the resulting emission.

\section{Influence of Entrainment on Jet Evolution}

In the previous sections, I focused on our current knowledge about the instabilities that can develop in relativistic jets and jet-obstacle interactions, as possible frames in which VHE photons could be produced. In this section, I summarize the global effect that either shear-layer entrainment or stellar mass-load (or both) can have on jet dynamics and propagation.

In a series of papers, Bicknell [28-32], De Young [33,34] and Komissarov [35-37] explained the transition from supersonic to transonic flows and the radiative properties of FRI jets at kiloparsec scales by means of turbulent mixing at the jet shear layer, although using different approaches and assumptions. De Young modeled entrainment and related the deceleration of jets to the properties of the host-galaxy ambient medium, using conservation equations [34]. Komissarov modeled the evolution of the post-deceleration (from relativistic to subrelativistic) flow in FRIs as a turbulent, low Mach number flow with thermal and relativistic populations coexisting and evolving (two-fluid model, [36,37]). The model allows for a conversion of thermal into relativistic particles via the coupling provided by turbulent dissipation. Bicknell [28] considered that the entrainment into a jet dominated by relativistic particles would convert it into a transonic, turbulent flow and applied this idea to derive the evolution of the electron distribution and magnetic field, to ultimately calculate the evolution in brightness of FRI radio-sources (assuming flux-freezing and adiabatic losses). The concept behind this model is the dissipation of kinetic energy via weak shocks and particle acceleration, modeled by a single losses term in the kinetic energy, plus a gain term in the heat equation. Later, the same author used conservation laws plus the source terms required to account for mass entrainment to model jet deceleration [31]. In the same way, Laing \& Bridle [24] explained the jet deceleration via entrainment. The models developed by [24,31] were later extended by Wang et al. [45] to study the development of 
the turbulent/shear layer towards the jet axis. In summary, the idea of entrainment, deceleration and dissipation caused by turbulence lays behind the evolution of FRI jets.

I observed, in Section 2, that this entrainment could be caused by small-scale $\mathrm{KH}$, RT or CF instabilities developing at the contact discontinuity between the jet and its environment (either a surrounding, slower and denser wind, or the backflow/cocoon generated by jet propagation). However, Komissarov [42] also proposed the possibility of mass entrainment by turbulent mixing at the cometary tails generated by jet-stellar wind interactions. The author also showed that the stellar mass input scenario could be considered to be a hydrodynamical problem, given the large size of the expected mixing region as compared to the particle gyroradii. Bowman, Leahy and Komissarov [43] performed steady-state simulations of jet evolution including a source term in the conservation equations to emulate the mass-load by stellar winds, considering an old, low-mass population, typical of giant elliptical galaxies that are the usual hosts of jets, with stellar winds $M_{w} \sim 10^{-12} \mathrm{M}_{\odot} / \mathrm{yr}$. The jets considered were very dilute $\left(10^{-31}-10^{-33} \mathrm{~g} / \mathrm{cm}^{3}\right)$ and hot $\left(10^{11}-10^{13} \mathrm{~K}\right)$. They found that these low power jets $\left(L_{j} \leq 10^{42} \mathrm{erg} / \mathrm{s}\right)$ could be decelerated by stellar winds alone, and a dual behavior in terms of internal energy/temperature: on the one hand, the hotter models cooled down as they expanded and were mass-loaded, whereas, on the other hand, colder models could gain temperature as they entrained and decelerated. The authors claimed that this was due to the dissipation caused by the mass-load.

Later, in [24], the authors estimated that the entrainment needed by jet deceleration in the radio galaxy 3C 31 (a powerful FRI jet, $L_{j} \sim 10^{44} \mathrm{erg} / \mathrm{s}$ could not be caused by stellar winds alone, but that continuous mixing at the shear layer was necessary. In contrast, Hubbard and Blackman [44] showed that a single, large star with a powerful wind, such as a Wolf-Rayet could possibly quench a low power jet $\left(10^{42} \mathrm{erg} / \mathrm{s}\right)$, as an extreme case, but that a collection of stars with weaker winds could efficiently decelerate the jet. They also derived an estimate of the distance at which the initial jet power is completely exhausted by the work done to push the entrained material, which can be expressed in terms of the stellar density and typical wind mass flux as (Perucho et al., in preparation):

$$
l_{\mathrm{d}} \simeq \frac{1}{\gamma_{\mathrm{j}}}\left(\frac{L_{\mathrm{j}}}{10^{43} \mathrm{erg} \mathrm{s}^{-1}}\right)\left(\frac{\dot{M}}{10^{-11} \mathrm{M}_{\odot} \mathrm{yr}^{-1}}\right)^{-1}\left(\frac{n_{\mathrm{s}}}{1 \mathrm{pc}^{-3}}\right)^{-1}\left(\frac{R_{\mathrm{j}}}{10 \mathrm{pc}}\right)^{-2} 10^{2} \mathrm{kpc},
$$

where $\gamma_{j}$ is the jet Lorentz factor, $L_{\mathrm{j}}$ is its kinetic power, $R_{j}$ is the jet radius, $\dot{M}$ is the mean mass-loss rate of the stellar population in the galaxy, and $n_{s}$ is the number of stars per unit volume. This last parameter is obviously a decreasing function of distance to the galactic nucleus, so the expression stands as a lower limit of the deceleration distance if $n_{S}$ is kept at its value in the galactic core.

These results anticipated that jet deceleration by stellar mass-load is sensitive to both jet power and the stellar population of the host galaxies. Perucho and collaborators [162] run RHD simulations of leptonic jets evolving in a galactic ambient medium, using the parameters and set up proposed in [43]. The results confirmed the general conclusions derived in the latter work, but also showed that jet disruption by stellar winds is a very sensitive process to the mean stellar wind mass fluxes. In other words, for each jet power there is a minimum mass-load rate required to produce a significant dynamic effect. Figure 9 shows the different effect of mass-load rates differing in a factor $10\left(10^{-11}\right.$ versus $10^{-12} \mathrm{M}_{\odot} / \mathrm{yr}$, [16]) on jets with the same kinetic power. The figure also shows the role of jet power, with jets of $L_{j}=10^{43} \mathrm{erg} / \mathrm{s}$ evolving without remarkable changes with or without mass-load (for the considered stellar wind mass fluxes), as opposed to the low power jets, $L_{j} \leq 10^{42} \mathrm{erg} / \mathrm{s}$.

It is relevant to remark that although these simulations introduce the mass-load by means of a source term in the mass conservation equation, the jet/obstacle interactions will induce strong inhomogeneities in jet pressure and velocity across it. The inhomogeneities will necessarily result in changes in emissivity and introduce small-scale instabilities. In [66,152], the authors evaluated the mass-load caused by the entrance of a star surrounded by its wind bubble into the jet, concluding that 
this process could cause enhanced entrainment at the jet shear-layer, if the wind bubble is destroyed before the jet flow reaches equilibrium with the stellar wind at $R_{S}$ (see Equation (21)).

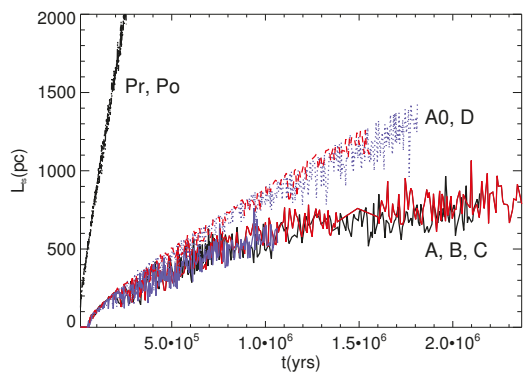

(a)

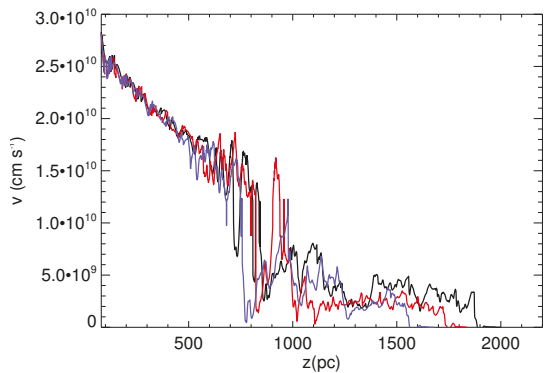

(b)

Figure 9. (a) Jet head position versus time for different mass-loaded jets. Pr, Po models correspond to jets with power $10^{44} \mathrm{erg} / \mathrm{s}$, model A0 is a low power jet $\left(\sim 10^{42} \mathrm{erg} / \mathrm{s}\right)$ with no mass-load, used as a fiducial simulation, models A, B, C and D have the same power as A0 but are mass-loaded by stars with $\dot{M} \simeq 10^{-12} \mathrm{M}_{\odot} / \mathrm{yr}$ (models $\mathrm{A}, \mathrm{B}$ and C) or $\dot{M} \simeq 10^{-13} \mathrm{M}_{\odot} / \mathrm{yr}$ (model D). Models $\mathrm{A}, \mathrm{B}$ and $\mathrm{C}$ only differ in the value of rest-mass density and temperature at injection, with increasing temperature and decreasing density from $A$ to $C$ (ranging from $3 \times 10^{11}$ to $3 \times 10^{13} \mathrm{~K}$ and $3 \times 10^{-33}$ to $3 \times 10^{-35} \mathrm{~g} / \mathrm{cm}^{3}$ ). The expansion velocity is clearly conditioned by entrainment, with one order of magnitude changes in mass-load implying critical changes in jet evolution. (b) Mean jet velocity along the first two kiloparsecs of evolution for models A, B and C (mass-loaded and decelerated). The disruption distance predicted by Equation (22) for these models is 200 pc. The difference between the prediction and the simulation is caused by the drop in stellar density with distance [162].

Finally, Perucho et al. (in preparation) ran a series of steady-state RMHD simulations of initially leptonic jets, making profit of a one-dimensional approximation to the RMHD equations developed by Komissarov et al. [163], including different mass-load rates, ambient density and stellar number distributions. This work confirms the possibility of efficient jet deceleration, even in the case of typical FRI jet powers of $L_{j} \sim 10^{43} \mathrm{erg} / \mathrm{s}$ depending on the stellar population in the host galaxy $\left(\dot{M} \geq 10^{-11} \mathrm{M}_{\odot} / \mathrm{yr}\right)$. Again, a dichotomy is found between jets that undergo heating and those that are initially hotter, and cool down as they evolve. Another relevant conclusion of that work is that loaded jets can significantly change their initial composition, and can even become proton dominated, even if not strongly decelerated.

\section{Discussion}

\subsection{Mass-Load and Dissipation}

Bicknell [28] and Komissarov [36] related the dissipation of kinetic energy to turbulence. Later, Bowman et al. [43] showed that entrainment could cause a drop in kinetic energy along with an increase of internal energy. Figures 6 and 10 show the relation between the deceleration region of FRI jets and diffuse emissivity that can be attached to dissipation of kinetic energy. In this section, I discuss this idea in the context of the FRI/FRII dichotomy and the production of VHE radiation in radio galaxies. The conservation Equations (6)-(8) that describe the evolution of a relativistic magnetized flow can be simplified, in the case of a steady-state, axisymmetric, and entraining jet propagating out of the galactic gravitational pull, with $v^{\phi}=v^{r}=0$ and $B^{r}=0$, to: 

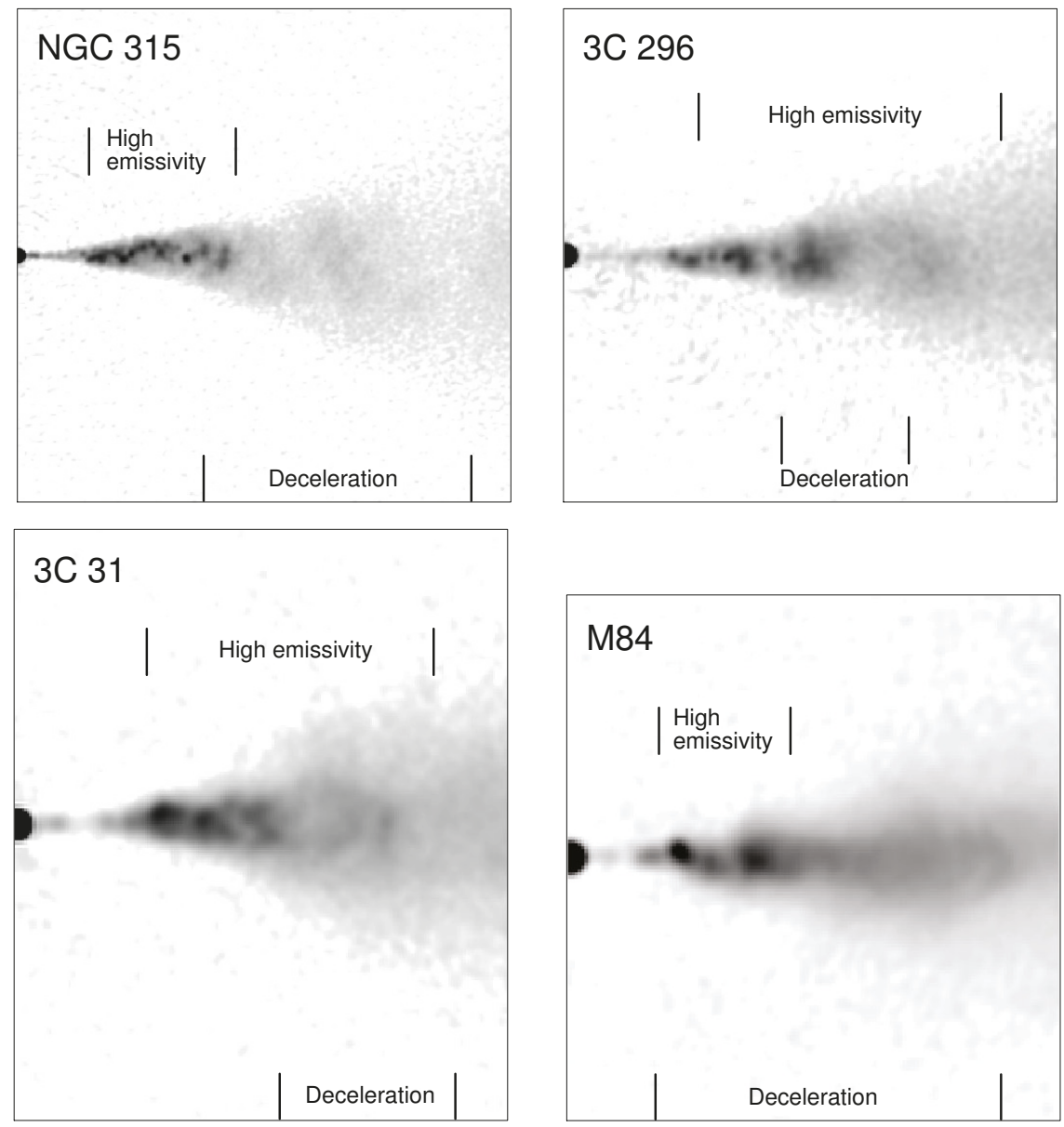

Figure 10. Grey-scale plots of intensity (VLA) for the inner regions of NGC 315 (top left), 3C 296 (top right), 3C 31 (bottom left) and M84 (bottom right). The image shows the coincidence of the deceleration region (as obtained from detailed modeling) and those of high-emissivity [27] (courtesy of Robert A. Laing).

$$
\begin{array}{r}
\partial_{z}\left(\gamma \rho v^{z}\right)=q, \\
\partial_{z}\left(\gamma \rho h^{*} v^{z} v^{z}+p^{*}-b^{z} b^{z}\right)=g^{z}, \\
\partial_{z}\left(\rho h^{*} \gamma^{2} v^{z}-b^{0} b^{z}-\gamma \rho v^{z}\right)=v^{z} g^{z},
\end{array}
$$

where $\partial_{z}$ is the partial derivative with respect to the axial coordinate, $q$ is the mass-load per unit volume and per unit area and time, and $g^{z}$ is the gravitational acceleration.

If we focus on the energy conservation along a portion of the jet with parameters $\rho, v^{z}, \gamma$, and $B^{\phi}$ at a given position $z_{0}$, write the terms involving the magnetic field in the observer's frame, neglect the gravitational pull and discretize the equation, we find that the changes in the flow are given by:

$$
\frac{\Delta\left[\left(\rho \gamma v^{z}\right)(h \gamma-1) R_{j}^{2}\right]}{\Delta z}+\frac{\Delta\left[\left(B^{\phi}\right)^{2} v^{z} R_{j}^{2}\right]}{\Delta z}=0 .
$$


Taking into account, from the mass-conservation equation, that $\Delta\left(\rho \gamma v^{z} R_{j}^{2}\right) / \Delta z=q R_{j}^{2}$ (for a small opening angle), and neglecting the transfer of magnetic energy flux (once the jet expansion is conical this can only occur via magnetic dissipation processes, which as stated above, are out of the ideal MHD approximation; see, e.g., [6] and references therein), we can drop the magnetic term from the equation and obtain:

$$
\left(\rho \gamma v^{z}\right)_{0} \frac{\Delta(h \gamma)}{\Delta z}=\left(1-(h \gamma)_{0}\right) q
$$

which can be rewritten as

$$
\frac{\Delta h}{h_{0}}=\frac{q \Delta z}{\left(\rho \gamma v^{z}\right)_{0}}\left(\frac{1}{(h \gamma)_{0}}-1\right)-\frac{\Delta \gamma}{\gamma_{0}} .
$$

This expression gives us an idea about the changes in the jet enthalpy as the jet flow evolves. We can now discuss the jet evolution in terms of $q /\left(\rho \gamma v^{z}\right)_{0}$, taking into account that $(\Delta \gamma) / \gamma_{0}$ is small $(\sim 1)$, and that $q /\left(\gamma \rho v^{z}\right)_{0}$ can be arbitrarily large (see also [43]):

1. Strong relative mass-load $\left(q \Delta z \gg\left(\gamma \rho v^{z}\right)_{0}\right)$ : in this case, $\Delta h<0$ and the conservation equation tells us that the initial jet enthalpy is transferred to the entrained flow.

2. Mild relative mass-load $\left(q \Delta z \sim\left(\gamma \rho v^{z}\right)_{0}\right)$ : in this case, $\Delta h$ could be both smaller than or larger than zero, depending on the value of the terms accounting for deceleration (so the initial jet enthalpy can grow).

3. Small relative mass-load $\left(q \Delta z \ll\left(\gamma \rho v^{z}\right)_{0}\right)$ : in this case, we could neglect the source term $q$ in the conservation equation above, and would be left with the Bernoulli expression for adiabatic evolution: $h \gamma=$ constant, where expansion of a hot jet flow translates into acceleration.

The third case could correspond to powerful jets, in which $q \Delta z \ll\left(\gamma \rho v^{z}\right)_{0}$, i.e., this is probably the case for FRIIs, as shown by numerical simulations of mass-entrainment by stellar winds in jets with different power [162], where it was reported that relatively weakly loaded jets do not suffer relevant dynamical effects (see Figure 9). In this case, dissipation of kinetic energy can occur at shocks (either stationary recollimation shocks or at traveling shocks) and the amount of dissipated energy (which is ultimately related to the emitted radiation) is regulated by the strength of these shocks, or by the growth of instabilities. In the case of recollimation shocks, these are stronger for hotter and slower flows, because the jet opening angle (determining the obliquity of the shock) increases with enthalpy and decreases with the Lorentz factor. Case 3 could thus be the scenario in FRII, where dissipation of kinetic energy occurs mainly at stationary shocks along the jet and at the interaction with the ambient medium (hotspot), close to which instabilities may have developed (e.g., Cygnus A).

Case 2 is a plausible mechanism for kinetic energy dissipation as long as the Lorentz factor is large enough to provide a significant contribution (in the terms that include $\Delta \gamma$ ). We could expect this heating process to play a role at the beginning of jet deceleration in FRIs ([43], Perucho et al., in preparation). Furthermore, the extra heating provided by mass-load naturally contributes to jet expansion, as observed in FRI jets from the so-called flaring point (e.g., [24]).

In case 1 , there is no possible gain of internal energy (via entrainment). We have to take into account that the jet is probably hot if the mass flux is small before entrainment begins $\left((\rho \gamma v)_{0} \ll\right)$. As a result of strong relative entrainment in a hot, dilute jet, it is rapidly cooled and decelerated (this is also derived from simulations, [16,43], Perucho et al., in preparation). Dissipation and particle acceleration must thus be triggered by turbulence and locally, at shocks. The question is whether the role played by radiative output through this fast cooling is enough to break the assumption of adiabatic evolution or not. It was actually reported that the flaring region in FRI jets differs from an adiabatic behavior [27]. Figure 11 shows the evolution of the energy fluxes (in logarithmic scale) for steady-state, axisymmetric jet simulations for hot dilute, magnetized jets mildly and strongly entrained (from Perucho et al., in preparation). These simulations show that entrainment always implies an increase of kinetic energy flux in jets that have a very small percentage of kinetic energy flux at injection (i.e., hot and/or strongly 
magnetized) despite the deceleration, because of the increase of rest-mass density. Depending on the relative role of entrainment, the kinetic energy can grow at the expense of internal energy flux alone (relatively mild entrainment), or at the expense of both internal and magnetic energy fluxes (strong entrainment). The authors reach the conclusion that jets are not heavily affected by mass-load as long as the initial mass flux per unit area at $z_{0}$ (with $v^{z} \simeq c$ ):

$$
\rho_{j, 0} \gamma_{j, 0}>6.7 \times 10^{-28}\left(\frac{\dot{M}}{10^{-11} \mathrm{M}_{\odot} \mathrm{yr}^{-1}}\right)\left(\frac{n_{s}}{1 \mathrm{pc}^{-3}}\right)\left(\frac{\Delta z}{1 \mathrm{kpc}}\right)^{3}\left(\frac{\tan (\alpha)}{\tan \left(1^{\circ}\right)}\right)^{2}\left(\frac{R_{j, 0}}{1 \mathrm{pc}}\right)^{-2}\left(\frac{v}{c}\right)^{-1} \mathrm{gcm}^{-3},
$$

where $\alpha$ is the jet opening angle. This expression does not take into account the role of magnetic tension, heating by dissipation of kinetic energy, the sudden increase of the jet opening angle once mass-loading starts to be relevant (because of the increased pressure), or the drop in stellar density along $\Delta z$. Nevertheless, it represents a good a priori estimate of the expected role that stellar mass-load can play on jet dynamics for given initial conditions.

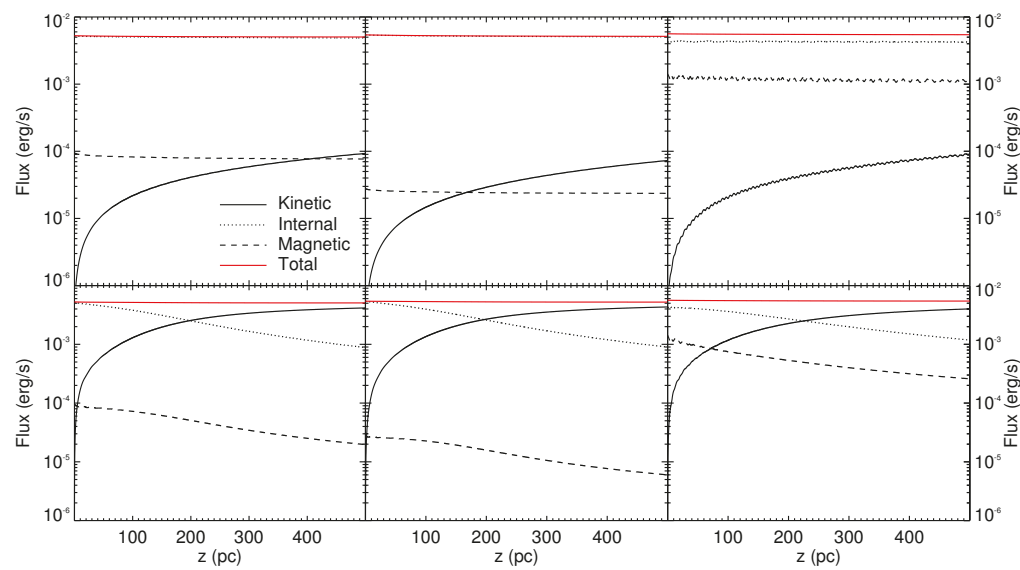

Figure 11. Evolution of the energy fluxes (in logarithmic scale) for steady-state, axisymmetric jet simulations for hot dilute, magnetized jets mildly (mean stellar mass-loss $\dot{M}_{w}=10^{-11} \mathrm{M}_{\odot} / \mathrm{yr}$, upper panels) and strongly mass-loaded $\left(\dot{M}_{w}=10^{-9} \mathrm{M}_{\odot} /\right.$ yr, lower panels). The jets also differ in the initial Lorentz factors 10 (left and right) and 6 (centre), and mean pitch angle (defined as $\tan ^{-1}\left(B^{\phi} / B^{z}\right)$ ) of $45^{\circ}$ (left and centre) and $75^{\circ}$ (right). The red line indicates the total flux, the dotted line stands the internal energy flux, the solid line the kinetic energy flux and the dashed line the magnetic energy flux. Perucho et al. (in preparation).

Both for cases 1 and 2, a cooled and decelerated jet leads to $h \gamma \rightarrow 1$, with the initial jet luminosity invested into kinetic energy flux (dominated by the entrained particles) and a part radiated away. In other words, the process of entrainment and deceleration leads to the depletion of the relativistic reservoir of the jet from parsec to kiloparsec scales. Once the jet becomes decelerated to subrelativistic speeds, the classical equations take over the description of jet evolution and the appropriate approach then becomes the one described in [28] and/or [36], including relevant terms such as buoyant forces and turbulent dissipation fed by further entrainment. Figure 12 shows a flow diagram of expanding jets, where I have indicated the processes that would correspond to the three mass-loading cases described here. Please note that this is independent from the origin of the entrainment. When following the diagram, it has to be kept in mind that different processes can take place at the same time. 


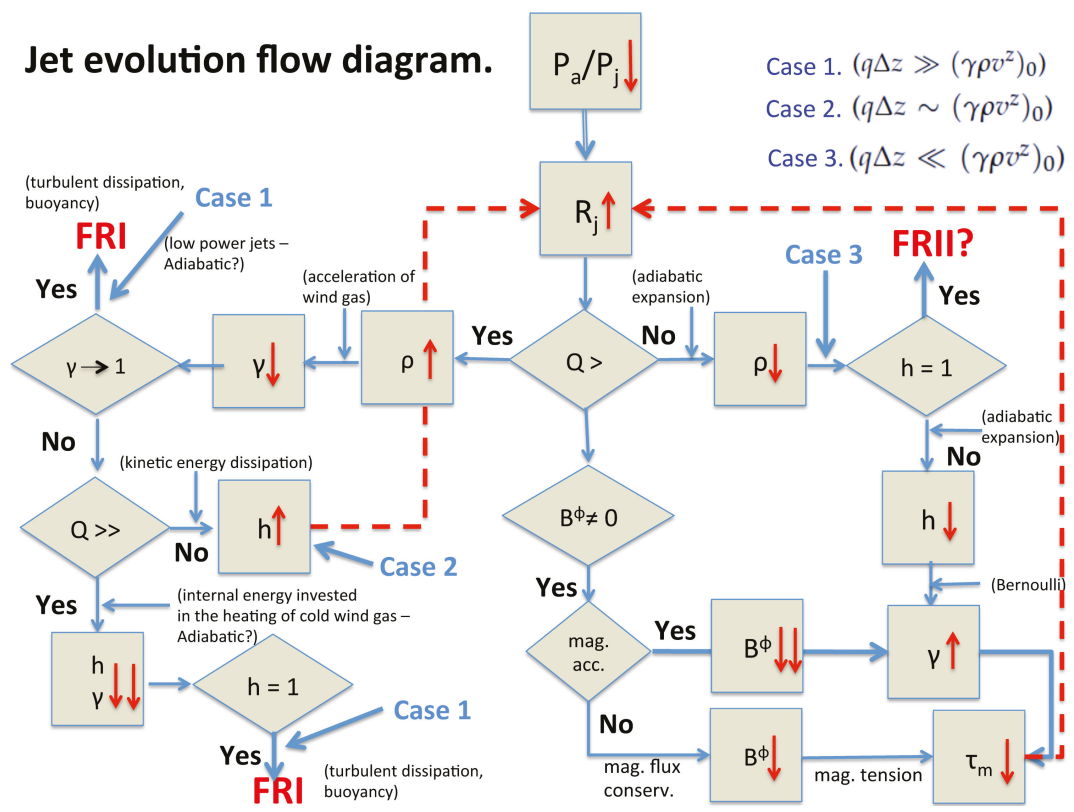

Figure 12. Flow diagram of jet evolution as it propagates through a galactic atmosphere. The rhombi indicate the bifurcations in the flow diagram depending on which processes dominate the dynamics, and the squares indicate the result of those processes. I have indicated the possible evolutionary options related to mass load in blue. Adapted from Perucho et al., in preparation (see also [164]). Please note that where case 1 is indicated, the jet evolution can be non-adiabatic [27]. Case 2 may imply particle acceleration and enhanced radiative output, still keeping an adiabatic behavior of the flow.

Finally, it is important to stress that independently from the dynamical role of entrainment, the entrainment of protons in the jet can significantly change the jet composition along its propagation.

\subsection{Jet Evolution: A Summary}

Jets are probably magnetically dominated at the formation region, with their mass flux dominated by pairs [4]. Magnetic acceleration and the Bernoulli effect convert part of the magnetic flux and internal energy into kinetic energy from the inner parsec to possibly hundreds of parsecs [165]. After the initial injection carves its way through the irregular inner galactic ambient medium, a channel forms that allows the particles to propagate at relativistic speeds along it. The CDI and KHI are assumed to develop at different scales along the jet, with the former acting while the jet is magnetically dominated and the KHI downstream of the magnetic acceleration region. The CDI (including the pressure-driven instability) could actually play a significant role in dissipating magnetic energy and trigger particle acceleration to relativistic energies, feeding the non-thermal population. As the jets expand in the external density/pressure gradients, the large-scale instabilities can be stabilized (see Section 2.3).

In parallel, as the jet flow propagates through the host galaxy, the interaction with stars and clouds can mass-load the jet, introducing protons and forcing the jet deceleration and the conversion of internal and/or magnetic energy flux into kinetic energy. In the case of fast jets, the deceleration caused by mass-load can produce an increase in the internal energy flux (e.g., [43,162], Perucho et al., in preparation). Deceleration of jets by stellar winds was proven to be efficient in low-power jets, 
even for old stellar populations with weak winds [162]. Jet/obstacle interactions also probably generate strong pressure and velocity inhomogeneities across the jet.

The expansion of a supersonic flow is followed by recollimation, a process that can trigger the development of RTI/CFI. Taking into account that these instabilities are enhanced in the case of larger opening angles $[85,108]$, we expect them to develop in slower and hotter jets (i.e, jets with smaller magnetosonic Mach numbers).

Although such short wavelength instabilities are favoured by the progressive deceleration that starts at the jet boundaries [27], other mechanisms can act in parallel, contributing to the FRI jet disruption process. Most FRI jets show a brightening region both in radio and X-rays, and, in some of them (see Figure 10 and, in particular, the case of NGC 315, discussed in [166]), there are clear hints of large-amplitude (i.e., probably disruptive) helical structures within the broader conically expanding jet, beyond the brightening region. Whether these helices are revealing the development of instabilities in the inner jet spine embedded within a broadening mixing layer is unclear, but it remains a plausible option. The symmetric brightening of the jet and counter-jet, prior to geometrical flaring could also be related a recollimation shock (e.g., [41]). The detection of high-energy emission from large-scales in FRIs requires that the decelerating process is accompanied by particle acceleration, and turbulence seems to be a good candidate to explain this.

Kinetically dominated jets (fast and cold) have smaller opening angles and dissipate kinetic energy mainly at the recollimation shocks and at their hotspots, but also possibly due to the development of small-scale instabilities in the boundary layer and at jet-obstacle interactions (see, e.g., [167] on Pictor A, where the X-rays were proposed to be synchrotron emission from the jet boundary layer). Although we do not observe clear hints of large-scale jet disruption at kpc-scales, we do observe helical structures (probably coupled to KHI modes) indicating that dissipation can take place both along the jet flow and at its head (via the dentist drill effect, $[128,129,168,169])$.

Recent simulations show that the propagation of FRII jets to hundreds of kiloparsecs can take place in 1-10 Myr, aided by the development through a decreasing density atmosphere and small scale oscillations of the jet head [20], until the oscillations grow to nonlinear amplitudes and then the dentist drill mechanism comes into play, decelerating the advance of the jet head. This could represent a transitory phase in the evolution of some FRII jets, most probably in those showing thin lobes and remarkable collimation. Instabilities would take long enough to grow to allow for large-scale jet propagation, and would start to contribute to strong kinetic energy dissipation only at those scales. Finally, from the lack of strong jet deceleration to subrelativistic speeds in FRII jets, we can infer that entrainment does not play a significant role in their evolution.

\subsection{A Final Comment on FRI/FRII Dichotomy and VHE Emission}

A conclusion that can be derived from this discussion is that dissipation is not acting as strongly in FRII jets as in FRIs at large scales. The dissipation in FRIs happens via processes that can significantly contribute to particle acceleration (e.g., strong shocks, shear, and turbulent mixing) and jet deceleration. Furthermore, larger opening angles in FRI jets after the flaring (which coincides with the beginning of deceleration, see, e.g., $[24,27]$ ) contribute to rapidly increase the entrainment rates and also the number of stars embedded in the jet, thus favoring further investment of jet kinetic flux into particle acceleration at interaction sites (e.g., [65]). Finally, deceleration can reduce the Doppler boosting and favor the detection in misaligned sources. In contrast, in more powerful FRII jets, the number of interactions is necessarily smaller because they are more collimated. Furthermore, the radiation that they trigger at interactions can be boosted far from our view. Altogether, this could contribute to explain why FRI radio galaxies are more numerous in the Fermi catalog than FRIIs, an observational fact that cannot be explained by means of simple population numbers (e.g., [48]).

The previous argumentation is valid for the diffuse HE radiation from FRI jets, and mass-loading was successfully used to reproduce the broad band synchrotron spectrum of radio galaxies from radio to X-rays, such as Centaurus A [64]. In fact, evidence is being found that shows the lack of correlation 
between Doppler boosting and gamma-ray emission in radiogalaxies ([170] and references therein), as opposed to blazars (see also [171,172]), where the fast variability of the detected VHE radiation seems to imply that it is generated at the most compact scales, via IC or even proton-proton interaction (see [49] for a recent review). This points towards a possible decoupling of the gamma-ray emission from radiogalaxies and their parsec-scale jets.

In this review, I have summarized candidate scenarios for energy dissipation and particle acceleration in radio galaxies. These scenarios are probably acting more efficiently in FRI jets, but the exact way in which $\mathrm{TeV}$ photons are produced in these sources remains to be determined, although it could be related to (1) enhanced efficiency (with respect to our current acceleration models) of particle acceleration in any of the aforementioned scenarios (via acceleration in turbulent flows), or (2) processes not taken into account in ideal RMHD, such as reconnection caused by the development of instabilities or jet-star/cloud interactions. The detailed multi-wavelength observational study and modeling of the dynamics of FRI radiogalaxies (e.g., [27,162], Perucho et al., in preparation) and nearby low-power, gamma-ray bright active galaxies like Centaurus A or NGC 1052 (e.g., [50,137-140,173-175]), plus particle-in-cell simulations (see, e.g., [176-178], and references therein), set the path to understanding the relation between jet deceleration and high-energy emission.

Funding: MP has been supported by the Spanish Ministerio de Ciencia y Universidades (grants AYA2015-66899-C2-1-P and AYA2016-77237-C3-3-P) and the Generalitat Valenciana (grant PROMETEOII/2014/069).

Acknowledgments: I thank José María Martí and Valentí Bosch-Ramon for the discussions that have contributed to the ideas that I expose in this review, and for comments to the original manuscript. I thank J. Matsumoto, K. Gourgouliatos, G. Bodo, M. Hardcastle, S. Komissarov, and R. Laing for giving permission to add figures for this publication. I also thank the graduate student Núria Chiquillo for providing tex equations that have eased the writing of part of the text.

Conflicts of Interest: The authors declare no conflict of interest. The funders had no role in the design of the study; in the collection, analyses, or interpretation of data; in the writing of the manuscript, or in the decision to publish the results.

\section{References}

1. Event Horizon Telescope Collaboration; Akiyama, K.; Alberdi, A.; Alef, W.; Asada, K.; Azulay, R.; Baczko, A.K.; Ball, D.; Baloković, M.; Barrett, J.; et al. First M87 Event Horizon Telescope Results. I. The Shadow of the Supermassive Black Hole. Astrophys. J. 2019, 875, L1. [CrossRef]

2. Event Horizon Telescope Collaboration; Akiyama, K.; Alberdi, A.; Alef, W.; Asada, K.; Azulay, R.; Baczko, A.K.; Ball, D.; Baloković, M.; Barrett, J.; et al. First M87 Event Horizon Telescope Results. V. Physical Origin of the Asymmetric Ring. Astrophys. J. 2019, 875, L5. [CrossRef]

3. Event Horizon Telescope Collaboration; Akiyama, K.; Alberdi, A.; Alef, W.; Asada, K.; Azulay, R.; Baczko, A.K.; Ball, D.; Baloković, M.; Barrett, J.; et al. First M87 Event Horizon Telescope Results. VI. The Shadow and Mass of the Central Black Hole. Astrophys. J. 2019, 875, L6. [CrossRef]

4. Blandford, R.D.; Znajek, R. Electromagnetic extraction of energy from Kerr black holes. Mon. Not. R. Astron. Soc. 1977, 179, 433-456. [CrossRef]

5. Tchekhovskoy, A.; Narayan, R.; Mckinney, J.C. Efficient generation of jets from magnetically arrested accretion on a rapidly spinning black hole. Mon. Not. R. Astron. Soc. 2011, 418, L79-L83. [CrossRef]

6. Komissarov, S.S. Central Engines. Acceleration, Collimation and Confinement of Jets. In Relativistic Jets from Active Galactic Nuclei; Boettcher, M., Harris, D.E., Krawczynski, H., Eds.; Wiley: Berlin, Germany, 2012; pp. 81-114.

7. Tchekhovskoy, A. Launching of Active Galactic Nuclei Jets. In The Formation and Disruption of Black Hole Jets; Contopoulos, I., Gabuzda, D., Kylafis, N., Eds.; Astrophysics and Space Science Library; Springer International: Cham, Switzerland, 2015; Volume 414, pp. 45-82.

8. Fanaroff, B.L.; Riley, J.M. The morphology of extragalactic radio sources of high and low luminosity. Mon. Not. R. Astron. Soc. 1974, 167, 31-36. [CrossRef]

9. Barkov, M.; Khangulyan, D.V. Direct wind accretion and jet launch in binary systems. Mon. Not. R. Astron. Soc. 2012, 421, 1351-1359. [CrossRef] 
10. Martí, J.; Luque-Escamilla, P.L.; Bosch-Ramon, V.; Paredes, J.M. A galactic microquasar mimicking winged radio galaxies. Nat. Co. 2017, 8, 1757. [CrossRef]

11. McNamara, B.R.; Nulsen, P.E.J. Heating hot atmospheres with active galactic nuclei. Ann. Rev. Astron. Astrophys. 2007, 45, 117-175. [CrossRef]

12. Fabian, A.C. Observational evidence of active galactic nuclei feedback. Ann. Rev. Astron. Astrophys. 2012, 50, 455-489. [CrossRef]

13. Perucho, M.; Quilis, V.; Martí, J.M. Intracluster medium reheating by relativistic jets. Astrophys. J. 2011, 743, 42-49. [CrossRef]

14. Wagner, A.Y.; Bicknell, G.V. Relativistic jet feedback in evolving galaxies. Astrophys. J. 2011, 728, 29-37. [CrossRef]

15. Wagner, A.Y.; Bicknell, G.V.; Umemura, M. Driving outflows with relativistic jets and the dependence of active galactic nucleus feedback efficiency on interstellar medium inhomogeneity. Astrophys. J. 2012, 757, 136-159. [CrossRef]

16. Perucho, M.; Martí, J.M.; Quilis, V.; Ricciardelli, E. Large-scale jets from active galactic nuclei as a source of intracluster medium heating: Cavities and shocks. Mon. Not. R. Astron. Soc. 2014, 445, 1462-1481. [CrossRef]

17. Mukherjee, D.; Bicknell, G.V.; Sutherland, R.; Wagner, A. Relativistic jet feedback in high-redshift galaxies-I. Dynamics. Mon. Not. R. Astron. Soc. 2016, 461, 967-983. [CrossRef]

18. Perucho, M.; Martí, J.M.; Quilis, V.; Borja-Lloret, M. Radio mode feedback: Does relativity matter? Mon. Not. R. Astron. Soc. 2017, 471, L120-L124. [CrossRef]

19. Bicknell, G.V.; Mukherjee, D.; Wagner, A.Y.; Sutherland, R.S.; Nesvadba, N.P.H. Relativistic jet feedback-II. Relationship to gigahertz peak spectrum and compact steep spectrum radio galaxies. Mon. Not. R. Astron. Soc. 2018, 475, 3493-3501. [CrossRef]

20. Perucho, M.; Martí, J.M.; Quilis, V. Long-term FRII jet evolution: Clues from three-dimensional simulations. Mon. Not. R. Astron. Soc. 2019, 482, 3718-3735. [CrossRef]

21. Ghisellini, G.; Celotti, A. The dividing line between FR I and FR II radio-galaxies. Astron. Astrophys. 2001, 379, L1-L4. [CrossRef]

22. Gopal-Krishna, Wiita, P.J. Extragalactic radio sources with hybrid morphology: Implications for the Fanaroff-Riley dichotomy. Astron. Astrophys. 2000, 363, 507-516.

23. Laing R.A. Large Scale Structure: Jets on kiloparsec Scales. In Energy Transport in Radio Galaxies and Quasars; Ekers, R.D., Fanti, C., Padrielli, L., Eds.; Proceedings of the 175th IAU Symp.; Kluwer Academic Publishers: Dordrecht, The Netherlands, 1996; pp. 147-152.

24. Laing R.A.; Bridle A.H. Relativistic models and the jet velocity field in the radio galaxy 3C 31. Mon. Not. R. Astron. Soc. 2002, 336, 328-352. [CrossRef]

25. Laing R.A.; Bridle A.H. Dynamical models for jet deceleration in the radio galaxy 3C 31. Mon. Not. R. Astron. Soc. 2002, 336, 1161-1180. [CrossRef]

26. Laing R.A.; Bridle A.H. Relativistic jet models for two low-luminosity radio galaxies: evidence for backflow? Mon. Not. R. Astron. Soc. 2012, 424, 1149-1169. [CrossRef]

27. Laing, R.A.; Bridle, A.H. Systematic properties of decelerating relativistic jets in low-luminosity radio galaxies. Mon. Not. R. Astron. Soc. 2014, 437, 3405-3441. [CrossRef]

28. Bicknell, G.V. A model for the surface brightness of a turbulent low Mach number jet. I-Theoretical development and application to 3C 31. Astrophys. J. 1984, 286, 68-87. [CrossRef]

29. Bicknell, G.V. A model for the surface brightness of a turbulent low Mach number jet. II-The global energy budget and radiative losses. Astrophys. J. 1986, 300, 591-604. [CrossRef]

30. Bicknell, G.V. A model for the surface brightness of a turbulent low Mach number jet. III-Adiabatic jets of arbitrary density ratio: Application to NGC 315. Astrophys. J. 1986, 305, 109-130. [CrossRef]

31. Bicknell, G.V. On the relationship between BL Lacertae objects and Fanaroff-Riley I radio galaxies. Astrophys. J. 1994, 422, 542-561. [CrossRef]

32. Bicknell, G.V. Relativistic jets and the Fanaroff-Riley classification of radio galaxies. Astrophys. J. Suppl. Ser. 1995, 101, 29-39. [CrossRef]

33. De Young, D.S. Mass entrainment in astrophysical jets. Astrophys. J. 1986, 307, 67-72. [CrossRef]

34. De Young, D.S. On the relation between Fanaroff-Riley Types I and II radio galaxies. Astrophys. J. 1993, 405, L13-L16. [CrossRef] 
35. Komissarov, S.S. Relativistic jet deceleration in weak extragalactic radio-sources. Astrophys. Space Sci. 1988, 150 59-64. [CrossRef]

36. Komissarov, S.S. A theoretical model of Fanaroff-Riley I jets. I-Equations of two-fluid approach and boundary conditions. Astrophys. Space Sci. 1990, 165 313-323. [CrossRef]

37. Komissarov, S.S. A theoretical model of Fanaroff-Riley I jets. II-Results of numerical calculations. Astrophys. Space Sci. 1990, 165 325-342. [CrossRef]

38. Rossi, P.; Mignone, A.; Bodo, G.; Massaglia, S.; Ferrari, A. Formation of dynamical structures in relativistic jets: the FRI case. Astron. Astrophys. 2008, 488, 795-806. [CrossRef]

39. Perucho, M.; Martí, J.M.; Cela, J.M.; Hanasz, M.; de la Cruz, R.; Rubio, F. Stability of three-dimensional relativistic jets: implications for jet collimation. Astron. Astrophys. 2010, 519, A41-A50. [CrossRef]

40. Tchekhovskoy, A.; Bromberg, O. Three-dimensional relativistic MHD simulations of Active Galactic Nuclei jets: Magnetic kink instability and Fanarofdf-Riley dichotomy. Mon. Not. R. Astron. Soc. 2016, 461, L46-L50. [CrossRef]

41. Perucho, M.; Martí, J.M. A numerical simulation of the evolution and fate of a Fanaroff-Riley type I jet. The case of 3C 31. Mon. Not. R. Astron. Soc. 2007, 382, 526-542. [CrossRef]

42. Komissarov, S.S. Mass-loaded relativistic jets. Mon. Not. R. Astron. Soc. 1994, 269, 394-402. [CrossRef]

43. Bowman, M.; Leahy, J.P.; Komissarov, S.S. The deceleration of relativistic jets by entrainment. Mon. Not. R. Astron. Soc. 1996, 279, 899-914. [CrossRef]

44. Hubbard, A.; Blackman, E.G. Active galactic nuclei jet mass loading and truncation by stellar winds. Mon. Not. R. Astron. Soc. 2006, 371, 1717-1721. [CrossRef]

45. Wang, Y.; Kaiser, C.R.; Laing, R.; Alexander, P.; Pavlovski, G.; Knigge, C. A relativistic mixing-layer model for jets in low-luminosity radio galaxies. Mon. Not. R. Astron. Soc. 2009, 397, 1113-1124. [CrossRef]

46. Kharb, P.; O’Dea, C.P.; Tilak, A.; Baum, S.A.; Haynes, E.; Noel-Storr, J.; Fallon, C.; Christiansen, K. VLBA and Chandra observations of jets in FRI radio galaxies: Constraints on jet evolution. Astrophys. J. 2012, 754, 1-17. [CrossRef]

47. Dermer, C.D.; Giebels, B. Active galactic nuclei at gamma-ray energies. C. R. Phys. 2016, 17, 594-616. [CrossRef]

48. Grandi, P. Gamma Rays from Radio Galaxies: FERMI/LAT Observations. Int. J. Mod. Phys. Conf. Ser. 2012, $8,25-30$.

49. Rieger, F.M.; Levinson, A. Radio Galaxies at VHE Energies. Galaxies 2018, 6, 116. [CrossRef]

50. The Fermi-LAT Collaboration. Fermi gamma-ray imaging of a radio galaxy. Science 2010, 328, 725-729. [CrossRef]

51. Rieger, F.M.; Mannheim, K. Particle acceleration in rotating and shearing jets from AGN. Astron. Astrophys. 2002, 396, 833-846. [CrossRef]

52. Stawarz, Ł, Ostrowski, M. Radiation from the relativistic jet: A role of the shear boundary layer. Astrophys. J. 2002, 578, 763-774. [CrossRef]

53. Rieger, F.M.; Duffy, P. Shear acceleration in relativistic astrophysical jets. Astrophys. J. 2004, 617, $155-161$. [CrossRef]

54. Lyutikov, M.; Ouyed, R. Inductive acceleration of UHECRs in sheared relativistic jets. Am. Phys. Soc. 2007, 27, 473-489. [CrossRef]

55. Sahayanathan, S. Boundary shear acceleration in the jet of MKN501. Mon. Not. R. Astron. Soc. 2009, 398, L49-L53. [CrossRef]

56. Rieger, F.M.; Duffy, P. Shear acceleration in expanding flows. Astrophys. J. 2016, 833, 34-39. [CrossRef]

57. Liu, R.-Y.; Rieger, F.M.; Aharonian, F.A. Particle acceleration in mildly relativistic shearing flows: The interplay of systematic and stochastic effects, and the origin of the extended high-energy emission in AGN jets. Astrophys. J. 2017, 842, 39-51. [CrossRef]

58. Kimura, S.S.; Murase, K.; Zhang, B.T. Ultrahigh-energy cosmic-ray nuclei from black hole jets: Recycling galactic cosmic rays through shear acceleration. Phys. Rev. D 2018, 97, 023026 [CrossRef]

59. Webb, G.M.; Barghouty, A.F.; Hu, Q.; le Roux, J.A. Particle acceleration due to cosmic-ray viscosity and fluid shear in astrophysical jets. Astrophys. J. 2018, 855, 31-53. [CrossRef]

60. Bednarek, W.; Protheroe, R.J. Gamma-rays from interactions of stars with active galactic nucleus jets. Mon. Not. R. Astron. Soc. 1997, 287, L9-L13. [CrossRef] 
61. Barkov, M.V.; Aharonian, F.A.; Bosch-Ramon, V. Gamma-ray flares from red giant/jet interactions in active galactic nuclei. Astrophys. J. 2010, 724, 1517-1523. [CrossRef]

62. Araudo, A.T.; Bosch-Ramon, V.; Romero, G. Gamma-ray emission from massive stars interacting with active galactic nuclei jets. Mon. Not. R. Astron. Soc. 2013, 436, 3626-3639. [CrossRef]

63. Wykes, S.; Croston, J.H.; Hardcastle, M.J.; Eilek, J.A.; Biermann, P.L.; Achterberg, A.; Bray, J.D.; Lazarian, A.; Haverkorn, M.; Protheroe, R.J.; et al. Mass entrainment and turbulence-driven acceleration of ultra-high energy cosmic rays in Centaurus A. Astron. Astrophys. 2013, 558, A19-A31. [CrossRef]

64. Wykes, S.; Hardcastle, M.J.; Karakas, A.I.; Vick, J.S. Internal entrainment and the origin of jet-related broad-band emission in Centaurus A. Mon. Not. R. Astron. Soc. 2015, 447, 1001-1013. [CrossRef]

65. Vieyro, F.L.; Torres-Albà, N.; Bosch-Ramon, V. Collective non-thermal emission from an extragalactic jet interacting with stars. Astron. Astrophys. 2017, 604, A57-A70. [CrossRef]

66. Torres-Albà, N.; Bosch-Ramon, V. Gamma rays from red giant wind bubbles entering the jets of elliptical host blazars. Astron. Astrophys. 2019, 623, A91-A98. [CrossRef]

67. Leismann, T.; Antón, L.; Aloy, M.A.; Müller, E.; Martí, J.M.; Miralles, J.A.; Ibáñez, J.M. Relativistic MHD simulations of extragalactic jets. Astron. Astrophys. 2005, 436, 503-526. [CrossRef]

68. Hardee, P.E. Stability properties of strongly magnetized spine-sheath relativistic jets. Astrophys. J. 2007, 664, 26-46. [CrossRef]

69. Bodo, G.; Mamatsashvili, G.; Rossi, P.; Mignone, A. Linear stability analysis of magnetized jets: the rotating case. Mon. Not. R. Astron. Soc. 2016, 462, 3031-3052. [CrossRef]

70. Hanasz, M.; Sol, H.; Sauty, C. On the local magnetorotational instability of astrophysical jets. Mon. Not. R. Astron. Soc. 2000, 316, 494-506. [CrossRef]

71. Begelman, M.C. Instability of toroidal magnetic field in jets and plerions. Astrophys. J. 1998, 493, 291-300. [CrossRef]

72. Turland, B.D.; Scheuer, P.A.G. Instabilities of Kelvin-Helmholtz type for relativistic streaming. Mon. Not. R. Astron. Soc. 1976, 176 421-441. [CrossRef]

73. Blandford, R.D.; Pringle, J.E. Kelvin-Helmholtz instability of relativistic beams. Mon. Not. R. Astron. Soc. 1976, 176, 443-454. [CrossRef]

74. Ferrari, A.; Trussoni, E.; Zaninetti, L. Relativistic Kelvin-Helmholtz instabilities in extragalactic radio-sources. Astron. Astrophys. 1979, 64, 43-52.

75. Hardee, P.E. On the configuration and propagation of jets in extragalactic radio sources. Astrophys. J. 1979, 234, 47-55. [CrossRef]

76. Ferrari, A.; Massaglia, S.; Trussoni, E. Magnetohydrodynamic Kelvin-Helmholtz instabilities in astrophysics. III-Hydrodynamic flows with shear layers. Mon. Not. R. Astron. Soc. 1982, 198, 1065-1079. [CrossRef]

77. Birkinshaw, M. The Kelvin-Helmholtz instability for relativistic particle beams. II-Flows bounded by a simple shear layer. Mon. Not. R. Astron. Soc. 1991, 252, 505-527. [CrossRef]

78. Hardee, P.E. On three-dimensional structures in relativistic hydrodynamic jets. Astrophys. J. 2000, 533, 176-193. [CrossRef]

79. Perucho, M.; Hanasz, M.; Martí, J.M.; Sol, H. Stability of hydrodynamical relativistic planar jets. I. Linear evolution and saturation of Kelvin-Helmholtz modes. Astron. Astrophys. 2004, 427, 415-430. [CrossRef]

80. Matsumoto, J.; Masada, Y. Two-dimensional numerical study for Rayleigh-Taylor and Richtmeyer-Meshkov instabilities in relativistic jets. Astrophys. J. 2013, 772, L1-L6. [CrossRef]

81. Matsumoto, J.; Aloy, M.A.; Perucho, M. Linear theory of the Rayleigh-Taylor instability at a discontinuous surface of a relativistic flow. Mon. Not. R. Astron. Soc. 2017, 472, 1421-1431. [CrossRef]

82. Toma, K.; Komissarov, S.S.; Porth, O. Rayleigh-Taylor instability in two-component relativistic jets. Mon. Not. R. Astron. Soc. 2017, 472, 1253-1258. [CrossRef]

83. Meliani, Z.; Keppens, R. Transverse stability of relativistic two-component jets. Astron. Astrophys. 2007, 475, 785-789. [CrossRef]

84. Meliani, Z.; Keppens, R. Decelerating relativistic two-component jets. Astrophys. J. 2009, 705, 1594-1606. [CrossRef]

85. Gourgouliatos, K.N.; Komissarov, S.S. Relativistic centrifugal instability. Mon. Not. R. Astron. Soc. 2018, 475, L125-L129. [CrossRef] 
86. Ferrari, A.; Trussoni, E.; Zaninetti, L. Magnetohydrodynamic Kelvin-Helmholtz instabilities in astrophysics. I-Relativistic flows-plane boundary layer in vortex sheet approximation. Mon. Not. R. Astron. Soc. 1980, 193, 469-486. [CrossRef]

87. Ferrari, A.; Trussoni, E.; Zaninetti, L. Magnetohydrodynamic Kelvin-Helmholtz instabilities in astrophysics. II Cylindrical boundary layer in vortex sheet approximation. Mon. Not. R. Astron. Soc. 1981, 196, 1051-1066. [CrossRef]

88. Bodo, G.; Mamatsashvili, G.; Rossi, P.; Mignone, A. Linear stability analysis of magnetized relativistic jets: The non-rotating case Mon. Not. R. Astron. Soc. 2013, 434, 3030-3046. [CrossRef]

89. Kim, J.; Balsara, D.S.; Lyutikov, M.; Komissarov, S.S. On the linear stability of magnetized jets without current sheets-relativistic case. Mon. Not. R. Astron. Soc. 2017, 467, 4647-4662. [CrossRef]

90. Kim, J.; Balsara, D.S.; Lyutikov, M.; Komissarov, S.S. On the linear stability of sheared and magnetized jets without current sheets-relativistic case. Mon. Not. R. Astron. Soc. 2018, 474, 3954-3966. [CrossRef]

91. Osmanov, Z.; Mignone, A.; Masaglia, S.; Bodo, G.; Ferrari, A. On the linear theory of Kelvin-Helmholtz instabilities of relativistic magnetohydrodynamic planar flows. Astron. Astrophys. 2008, 490, 493-500. [CrossRef]

92. Istomin, Y.N.; Pariev, V.I. Stability of a relativistic rotating electron-positron jet. Mon. Not. R. Astron. Soc. 1994, 267, 629-636. [CrossRef]

93. Istomin, Y.N.; Pariev, V.I. Stability of a relativistic rotating electron-positron jet: Non-axisymmetric perturbations. Mon. Not. R. Astron. Soc. 1996, 281, 1-26. [CrossRef]

94. Bodo, G.; Mamatsashvili, G.; Rossi, P.; Mignone, A. Linear stability analysis of magnetized relativistic rotating jets. Mon. Not. R. Astron. Soc. 2019, 485, 2909-2921. [CrossRef]

95. Lyubarskii, Y.E. Kink instability of relativistic force-free jets. Mon. Not. R. Astron. Soc. 1999, 308, 1006-1010. [CrossRef]

96. Gourgouliatos, K.N.; Fendt, C.; Clausen-Brown, E.; Lyutikov, M. Magnetic field structure of relativistic jets without current sheets. Mon. Not. R. Astron. Soc. 2012, 419, 3048-3059. [CrossRef]

97. Martí, J.M. The structure of steady, relativistic, magnetized jets with rotation. Mon. Not. R. Astron. Soc. 2015, 452, 3106-3123. [CrossRef]

98. Martí, J.M.; Perucho, M.; Gómez, J.L. The internal structure of overpressured, magnetized, relativistic jets. Astrophys. J. 2016, 831, 163-177. [CrossRef]

99. Urpin, V. Instability of relativistic sheared jets and distinction between FRI and FRII sources. Astron. Astrophys. 2002, 385, 14-20. [CrossRef]

100. Perucho, M.; Martí, J.M.; Hanasz, M. Nonlinear stability of relativistic sheared planar jets. Astron. Astrophys. 2005, 443, 863-881. [CrossRef]

101. Perucho, M.; Hanasz, M.; Martí, J.M.; Miralles, J.A. Resonant Kelvin-Helmholtz modes in sheared relativistic flows. Phys. Rev. E 2007, 75, 056312. [CrossRef]

102. Hardee, P.E. Helical twisting on an adiabatically expanding jet. II Spatial growth. Astrophys. J. 1986, 303, 111-118. [CrossRef]

103. Ferrari, A.; Trussoni, E.; Zaninetti, L. Magnetohydrodynamic instabilities and electron acceleration in extended extragalactic radio sources. Astron. Astrophys. 1979, 79, 190-196.

104. Mizuno, Y.; Gómez, J.; Nishikawa, K.-I.; Meli, A.; Hardee, P.E.; Rezzolla, L.; Singh, C.; Pino, E. Magnetic dissipation in relativistic jets. Galaxies 2016, 4, 40. [CrossRef]

105. Hardee, P.E. Helical twisting on an adiabatically expanding jet. I Propagation and temporal growth. Astrophys. J. 1984, 287, 523-533. [CrossRef]

106. Hardee, P.E. Spatial stability of relativistic jets-Application to 3C 345. Astrophys. J. 1987, 318, 78-92. [CrossRef]

107. Porth, O.; Komissarov, S.S. Causality and stability of cosmic jets. Mon. Not. R. Astron. Soc. 2015, 452, 1089-1104. [CrossRef]

108. Gourgouliatos, K.N.; Komissarov, S.S. Reconfinement and loss of stability in jets from active galactic nuclei. Nat. Astron. 2018, 2, 167-171. [CrossRef]

109. Blandford, R.D.; Payne, D.G. Hydromagnetic flows from accretion disks and the production of radio jets. Mon. Not. R. Astron. Soc. 1982, 199, 883-903. [CrossRef] 
110. Kim, J.; Balsara, D.S.; Lyutikov, M.; Komissarov, S.S.; George, D.; Siddireddy, P.K. On the linear stability of magnetized jets without current sheets-Non-relativistic case. Mon. Not. R. Astron. Soc. 2015, 450, 982-997. [CrossRef]

111. Mizuno, Y.; Lyubarsky, Y.; Nishikawa, K.-I.; Hardee, P.E. Three-dimensional relativistic magnetohydrodynamic simulations of current-driven instability. I. Instability of a static column. Astrophys. J. 2009, 700, 684-693. [CrossRef]

112. Mizuno, Y.; Hardee, P.E.; Nishikawa, K.-I. Three-dimensional relativistic magnetohydrodynamic simulations of current-driven instability with a sub-Alfvénic jet: temporal properties. Astrophys. J. 2011, 734, 19-36. [CrossRef]

113. Mizuno, Y.; Hardee, P.E.; Nishikawa, K.-I. Spatial growth of the current-driven instability in relativistic jets. Astrophys. J. 2014, 784, 167-182. [CrossRef]

114. Kersalé, E.; Longaretti, P.-Y.; Pelletier, G. Pressure- and magnetic shear-driven instabilities in rotating MHD jets. Astron. Astrophys. 2000, 363, 1166-1176.

115. Longaretti, P.-Y. Pressure-driven instabilities in astrophysical jets. Lect. Not. Phys. 2008, 754 131-151.

116. Tomimatsu, A.; Matsuoka, T.; Masaaki, T. Screw instability in black hole magnetospheres and a stabilizing effect of field-line rotation. Phys. Rev. D 2001, 64, 123003. [CrossRef]

117. Millas, D.; Keppens, R.; Meliani, Z. Rotation and toroidal magnetic field effects on the stability of two-component jets. Mon. Not. R. Astron. Soc. 2017, 470, 592-605. [CrossRef]

118. Lyutikov, M.; Pariev, V.I.; Gabuzda, D.C. Polarization and structure of relativistic parsec-scale AGN jets. Mon. Not. R. Astron. Soc. 2005, 360, 869-891. [CrossRef]

119. Fuentes, A.; Gómez, J.L.; Martí, J.M.; Perucho, M. Total and linearly polarized synchrotron emission from overpressured magnetized relativistic jets. Astrophys. J. 2018, 860, 121-146. [CrossRef]

120. Kadler, M.; Ros, E.; Perucho, M. The trails of superluminal jet components in 3C 111. Astrophys. J. 2008, 680, 867-884. [CrossRef]

121. Bridle, A.H.; Perley, R.A. Extragalactic radio jets. Ann. Rev. Astron. Astrophys. 1984, 22, 31-358. [CrossRef]

122. Bridle, A.H.; Hough, D.H.; Lonsdale, C.J.; Burns, J.O.; Laing, R.A. Deep VLA imaging of twelve extended 3CR quasars. Astron. J. 1994, 108, 766-820. [CrossRef]

123. Beuchert, T.; Kadler, M.; Perucho, M.; Großberger, C.; Schulz, R.; Agudo, I.; Casadio, C.; Gómez, J.L.; Gurwell, M.; Homan, D.; et al. VLBA polarimetric monitoring of 3C 111. Astron. Astrophys. 2018, 610, A32. [CrossRef]

124. Lobanov, A.P.; Zensus, J.A. A cosmic double helix in the archetypical quasar 3C273. Science 2001, 294, 128-131. [CrossRef] [PubMed]

125. Hardee, P.E. Modeling helical structures in relativistic jets. Astrophys. J. 2003, 597, 798-808. [CrossRef]

126. Hardee, P.E.; Walker, R.C.; Gómez, J.L. Modeling the 3C 120 radio jet from 1 to 30 Milliarcseconds. Astrophys. J. 2005, 620, 646-664. [CrossRef]

127. Hardee, P.E.; Eilek, J.A. Using twisted filaments to model the inner jet in M 87. Astrophys. J. 2011, 735, 61-80. [CrossRef]

128. Perucho, M.; Kovalev, Y.Y.; Lobanov, A.P.; Hardee, P.E.; Agudo, I. Anatomy of helical extragalactic jets: The case of S5 0836+710. Astrophys. J. 2012, 749, 55-72. [CrossRef]

129. Vega-García, L.; Perucho, M.; Lobanov, A. Derivation of the physical parameters of the jet in S5 $0836+710$ from stability analysis. Astron. Astrophys. 2019, 627, A79-A94. [CrossRef]

130. Perucho, M.; Lobanov, A.P.; Martí, J.M.; Hardee, P.E. The role of Kelvin-Helmholtz instability in the internal structure of relativistic outflows. The case of the jet in 3C 273. Astron. Astrophys. 2006, 456, 493-504. [CrossRef]

131. Hanasz, M. Kelvin-Helmholtz instability of relativistic jets-The transition from linear to nonlinear regime. In Relativistic Jets in AGNs, Proceedings of the International Conference; Ostrowski, M., Sikora, M., Madejski, G., Begelman, M., Eds.; Centre for Astronomy, Nicolaus Copernicus University: Łysomice, Poland, 1997; pp. 85-89.

132. Perucho, M.; Martí, J.M.; Hanasz, M. Stability of hydrodynamical relativistic planar jets. II. Long-term nonlinear evolution. Astron. Astrophys. 2004, 427, 431-444. [CrossRef]

133. Payne, D.G.; Cohn, H. The stability of confined radio jets-The role of reflection modes. Astrophys. J. 1985, 291, 655-667. [CrossRef] 
134. Hardee, P.E.; Hughes, P.A. The effect of external winds on relativistic jets. Astrophys. J. 2003, 583, $116-123$. [CrossRef]

135. Mizuno, Y.; Hardee, P.E.; Nishikawa, K.-I. Three-dimensional relativistic magnetohydrodynamic simulations of magnetized spine-sheath relativistic jets. Astrophys. J. 2007, 662, 835-850. [CrossRef]

136. Blandford, R.D.; Koenigl, A. A model for the knots in the M87 jet. Astrophys. Lett. 1979, 20, 15-21.

137. Müller, C.; Kadler, M.; Ojha, R.; Perucho, M.; Großberger, C.; Ros, E.; Wilms, J.; Blanchard, J.; Böck, M.; Carpenter, B.; et al. TANAMI monitoring of Centaurus A: The complex dynamics in the inner parsec of an extragalactic jet. Astron. Astrophys. 2014, 569, A115. [CrossRef]

138. Hardcastle, M.J.; Croston, J.H. Modelling TeV ?-ray emission from the kiloparsec-scale jets of Centaurus A and M87. Mon. Not. R. Astron. Soc. 2011, 415, 133-142. [CrossRef]

139. Worrall, D.M.; Birkinshaw, M.; Kraft, R.P.; Sivakoff, G.R.; Jordán, A.; Hardcastle, M.J.; Brassington, N.J.; Croston, J.H.; Evans, D.A.; Forman, W.R.; et al. Where Centaurus A gets its X-Ray knottiness. Astrophys. J. 2008, 673, L135-L138. [CrossRef]

140. Goodger, J.L.; Hardcastle, M.; Croston, J.H.; Kraft, R.P.; Birkinshaw, M.; Evans, D.A.; Jordán, A.; Nulsen, P.E.J.; Sivakoff, G.R.; Worrall, D.M.; et al. Long-term monitoring of the dynamics and particle acceleration of knots in the jet of Centaurus A. Astrophys. J. 2010, 708, 675-697. [CrossRef]

141. Bosch-Ramon, V. Non-thermal emission from standing relativistic shocks: An application to red giant winds interacting with AGN jets. Astron. Astrophys. 2015, A575, 109-114. [CrossRef]

142. Aharonian, F.; Akhperjanian, A.G.; Bazer-Bachi, A.R.; Beilicke, M.; Benbow, W.; Berge, D.; Bernlöhr, K.; Boisson, C.; Bolz, O.; Borrel, V.; et al. Fast variability of Tera-Electron Volt $\gamma$ rays from the radio galaxy M87. Science 2006, 314, 1424-1427. [CrossRef]

143. Albert, J.; Aliu, E.; Anderhub, H.; Antonelli, L.A.; Antoranz, P.; Backes, M.; Baixeras, C.; Barrio, J.A.; Bartko, H.; Bastieri, D.; et al. Very high energy gamma-ray observations of strong flaring activity in M87 in 2008 February. Astrophys. J. 2008, 685, L23-L26. [CrossRef]

144. VERITAS Collaboration. Radio imaging of the very-high-energy $\gamma$-ray emission region in the central engine of a radio galaxy. Science 2009, 325, 444-448. [CrossRef]

145. Aliu, E.; Arlen, T.; Aune, T.; Beilicke, M.; Benbow, W.; Bouvier, A.; Bradbury, S.M.; Buckley, J.H.; Bugaev, V.; Byrum, K.; et al. VERITAS Observations of day-scale flaring of M 87 in 2010 April. Astrophys. J. 2012, 746, 141-147. [CrossRef]

146. Abramowski, A.; Acero, F.; Aharonian, F.; Akhperjanian, A.G.; Anton, G.; Balzer, A.; Barnacka, A.; De Almeida, U.B.; Becherini, Y.; Becker, J.; et al. The 2010 very high energy $\gamma$-ray flare and 10 Years of multi-wavelength observations of M 87. Astrophys. J. 2012, 746, 151-158. [CrossRef]

147. Barkov, M.V.; Bosch-Ramon, V.; Aharonian, F.A. Interpretation of the flares of M87 at TeV energies in the cloud-jet Interaction scenario. Astrophys. J. 2012, 755, 170-177. [CrossRef]

148. Khangulyan, D.V.; Barkov, M.V.; Bosch-Ramon, V.; Aharonian, F.A.; Dorodnitsyn, A.V. Star-Jet Interactions and Gamma-Ray Outbursts from 3C454.3. Astrophys. J. 2013, 774, 113-127. [CrossRef]

149. Zacharias, M.; Böttcher, M.; Jankowsky, F.; Lenain, J.P.; Wagner, S.J.; Wierzcholska, A. Cloud Ablation by a Relativistic Jet and the Extended Flare in CTA 102 in 2016 and 2017. Astrophys. J. 2017, 851, 72-80. [CrossRef]

150. Bosch-Ramon, V.; Perucho, M.; Barkov, M.V. Clouds and red giants interacting with the base of AGN jets. Astron. Astrophys. 2012, 539, A69-A80. [CrossRef]

151. De la Cita, V.M.; Bosch-Ramon, V.; Paredes-Fortuny, X.; Khangulyan, D.; Perucho, M. Coupling hydrodynamics and radiation calculations for star-jet interactions in active galactic nuclei. Astron. Astrophys. 2016, A591, 15-27. [CrossRef]

152. Perucho, M.; Bosch-Ramon, V.; Barkov, M.V. Impact of red giant/AGB winds on active galactic nucleus jet propagation. Astron. Astrophys. 2017, 606, A40-A53. [CrossRef]

153. Perucho, M.; Bosch-Ramon, V. 3D simulations of microquasar jets in clumpy stellar winds. Astron. Astrophys. 2012, 539, A57-A71. [CrossRef]

154. Vieyro, F.L.; Bosch-Ramon, V.; Torres-Albà, N. Non-thermal emission resulting from a supernova explosion inside an extragalactic jet. Astron. Astrophys. 2019, 622, A175. [CrossRef]

155. Tavecchio, F.; Ghisellini, G. On the magnetization of BL Lac jets. Mon. Not. R. Astron. Soc. 2016, 456, 2374-2382. [CrossRef]

156. Giannios, D.; Uzdensky, D.A.; Begelman, M.C. Fast TeV variability in blazars: Jets in a jet. Mon. Not. R. Astron. Soc. 2009, 395, L29-L33. [CrossRef] 
157. Kadler, M.; Krauss, F.; Mannheim, K.; Ojha, R.; Müller, C.; Schulz, R.; Anton, G.; Baumgartner, W.; Beuchert, T.; Buson, S.; et al. Coincidence of a high-fluence blazar outburst with a PeV-energy neutrino event. Nat. Phys. 2016, 12, 807-814. [CrossRef]

158. IceCube Collaboration. Neutrino emission from the direction of the blazar TXS 0506+056 prior to the IceCube-170922A alert. Science 2018, 361, 147-151.

159. Keivani, A.; Murase, K.; Petropoulou, M.; Fox, D.B.; Cenko, S.B.; Chaty, S.; Coleiro, A.; DeLaunay, J.J.; Dimitrakoudis, S.; Evans, P.A.; et al. A multimessenger picture of the flaring blazar TXS 0506+056: Implications for high-energy neutrino emission and cosmic-ray acceleration. Astrophys. J. 2018, 864, 84-99. [CrossRef]

160. Murase, K.; Oikonomou, F.; Petropoulou, M. Blazar flares as an origin of high-energy cosmic neutrinos? Astrophys. J. 2018, 865, 124-133. [CrossRef]

161. Sahakyan, N. Lepto-hadronic $\gamma$-Ray and neutrino emission from the jet of TXS 0506+056. Astrophys. J. 2018, 866, 109-114. [CrossRef]

162. Perucho, M.; Martí, J.M.; Laing, R.A.; Hardee, P.E. On the deceleration of Fanaroff-Riley Class I jets: Mass loading by stellar winds. Mon. Not. R. Astron. Soc. 2014, 441, 1488-1503. [CrossRef]

163. Komissarov, S.S.; Porth, O.; Lyutikov, M. Stationary relativistic jets. ComAC 2015, 2, 9-24. [CrossRef]

164. Perucho, M. Radio source evolution and the interplay with the host galaxy. Proc. Sci. 2019. [CrossRef]

165. Homan, D.C.; Lister, M.L.; Kovalev, Y.Y.; Pushkarev, A.B.; Savolainen, T.; Kellermann, K.I.; Richards, J.L.; Ros, E. MOJAVE. XII. Acceleration and Collimation of Blazar Jets on Parsec Scales. Astrophys. J. 2015, 798, 134-149. [CrossRef]

166. Worrall, D.M.; Birkinshaw, M.; Laing, R.A.; Cotton, W.D.; Bridle, A.H. The inner jet of radio galaxy NGC 315 as observed with Chandra and the Very Large Array. Mon. Not. R. Astron. Soc. 2007, 380, 2-14. [CrossRef]

167. Hardcastle, M.J.; Lenc, E.; Birkinshaw, M.; Croston, J.H.; Goodger, J.L.; Marshall, H.L.; Perlman, E.S.; Siemiginowska, A.; Stawarz, Ł.; Worrall, D.M. Deep Chandra observations of Pictor A. Mon. Not. R. Astron. Soc. 2016, 455, 3526-3545. [CrossRef]

168. Scheuer, P.A.G. Morphology and power of radio sources. In Extragalactic Radio Sources; Proceedings of the Symposium; D. Reidel Publishing Co.: Dordrecht, The Netherlands, 1982; pp. 163-165.

169. Perucho, M.; Martí-Vidal, I.; Lebanon, A.P.; Hardee, P.E. S5 0836+710: An FRII jet disrupted by the growth of a helical instability? Astron. Astrophys. 2012, 545, A65-A68. [CrossRef]

170. Angioni, R.; Ros, E.; Kadler, M.; Ojha, R.; Müller, C.; Edwards, P.G.; Burd, P.R.; Carpenter, B.; Dutka, M.S.; Gulyaev, S.; et al. Gamma-ray emission in radio galaxies under the VLBI scope. I. Parsec-scale jet kinematics and high-energy properties of $\gamma$-ray-detected TANAMI radio galaxies. Astron. Astrophys. 2019, 627, A148-A182. [CrossRef]

171. Arshakian, T.G.; León-Tavares, J.; Böttcher, M.; Torrealba, J.; Chavushyan, V.H.; Lister, M.L.; Ros, E.; Zensus, J.A. Radio-optical-gamma-ray properties of MOJAVE AGN detected by Fermi/LAT. Astron. Astrophys. 2012, 537, A32-A41. [CrossRef]

172. Böck, M.; Kadler, M.; Müller, C.; Tosti, G.; Ojha, R.; Wilms, J.; Bastieri, D.; Burnett, T.; Carpenter, B.; Cavazzuti, E.; et al. Radio and gamma-ray properties of extragalactic jets from the TANAMI sample. Astron. Astrophys. 2016, 590, A40-A51. [CrossRef]

173. Fromm, C.M.; Perucho, M.; Porth, O.; Younsi, Z.; Ros, E.; Mizuno, Y.; Zensus, J.A.; Rezzolla, L. Jet-torus connection in radio galaxies. Relativistic hydrodynamics and synthetic emission. Astron. Astrophys. 2018, 609, A80-A85. [CrossRef]

174. Baczko, A.-K.; Schulz, R.; Kadler, M.; Ros, E.; Perucho, M.; Fromm, C.M.; Wilms, J. Asymmetric jet production in the active galactic nucleus of NGC 1052. Astron. Astrophys. 2019, 623, A27-A45. [CrossRef]

175. Fromm, C.M.; Younsi, Z.; Bazcko, A.; Mizuno, Y.; Porth, O.; Perucho, M.; Olivares, H.; Nathanail, A.; Angelakis, E.; Ros, E.; et al. Using evolutionary algorithms to model relativistic jets: Application to NGC 1052. Astron. Astrophys. 2019. [CrossRef]

176. Nishikawa,K.-I.; Mizuno, Y.; Gómez, J.L.; Dutan, I.; Meli, A.; Niemiec, J.; Kobzar, O.; Pohl, M.; Sol, H.; MacDonald, N.; et al. Relativistic Jet Simulations of the Weibel Instability in the Slab Model to Cylindrical Jets with Helical Magnetic Fields. Galaxies 2019, 7, 29. [CrossRef]

177. Martí, J.M. Numerical Simulations of Jets from Active Galactic Nuclei. Galaxies 2019, 7, 24. [CrossRef] 
178. Wong, K.; Zhdankin, V.; Uzdensky, D.A.; Werner, G.R.; Begelman, M.C. First-principles demonstration of diffusive particle acceleration in kinetic simulations of relativistic plasma turbulence. arXiv 2019, arXiv:1901.03439.

(c) (1)

(C) 2019 by the author. Licensee MDPI, Basel, Switzerland. This article is an open access article distributed under the terms and conditions of the Creative Commons Attribution (CC BY) license (http://creativecommons.org/licenses/by/4.0/). 


\title{
Radio Galaxies-The TeV Challenge
}

\author{
Bindu Rani ${ }^{\dagger}$ \\ NASA Goddard Space Flight Center, Greenbelt, MD 20771, USA; bindu.rani@nasa.gov \\ + NPP Fellow.
}

Received: 1 November 2018; Accepted: 15 January 2019; Published: 22 January 2019

\begin{abstract}
Over the past decade, our knowledge of the $\gamma$-ray sky has been revolutionized by groundand space-based observatories by detecting photons up to several hundreds of tera-electron volt (TeV) energies. A major population of the $\gamma$-ray bright objects are active galactic nuclei (AGN) with their relativistic jets pointed along our line-of-sight. Gamma-ray emission is also detected from nearby misaligned AGN such as radio galaxies. While the TeV-detected radio galaxies (TeVRad) only form a small fraction of the $\gamma$-ray detected AGN, their multi-wavelength study offers a unique opportunity to probe and pinpoint the high-energy emission processes and sites. Even in the absence of substantial Doppler beaming TeVRad are extremely bright objects in the TeV sky (luminosities detected up to $10^{45} \mathrm{erg} \mathrm{s}^{-1}$ ), and exhibit flux variations on timescales shorter than the event-horizon scales (flux doubling timescale less than $5 \mathrm{~min}$ ). Thanks to the recent advancement in the imaging capabilities of high-resolution radio interferometry (millimeter very long baseline interferometry, mm-VLBI), one can probe the scales down to less than 10 gravitational radii in TeVRad, making it possible not only to test jet launching models but also to pinpoint the high-energy emission sites and to unravel the emission mechanisms. This review provides an overview of the high-energy observations of TeVRad with a focus on the emitting sites and radiation processes. Some recent approaches in simulations are also sketched. Observations by the near-future facilities like Cherenkov Telescope Array, short millimeter-VLBI, and high-energy polarimetry instruments will be crucial for discriminating the competing high-energy emission models.
\end{abstract}

Keywords: active galactic nuclei; radio galaxies; gamma-rays; jets

\section{Introduction}

An exciting discovery enabled by space- and ground-based high-energy observations is the detection of $\gamma$-rays from over 3000 extragalactic sources. Active galactic nuclei (AGN, powered by accretion onto a few million to several billion solar mass black holes) form a major population of the $\gamma$-ray sky. AGN often produce collimated outflows, called relativistic jets (see Figure 1), whose beams of radiation pierce through the Universe and reach us from the past, offering a unique opportunity to study the Universe when it was an order of magnitude younger than today. Despite the intensive study of AGN, the location and origin of their $\gamma$-rays remains a mystery. Some studies imply the emission region is located close to the central black hole [1-5], while there are counterexamples which argue for $\gamma$-rays coming from farther out in the jet [6-8]. Extremely bright $\gamma$-ray flashes in AGN on time scales of minutes to hours have attracted the attention of the entire astronomical community, as this suggests that the particles that produce $\gamma$-rays can be accelerated with enormous efficiency in tiny regions within more extended sources. The question of what powers $\gamma$-ray AGN flares is ultimately related to the energy dissipation mechanism at work in their relativistic jets.

AGN jets are among the largest and most efficient particle accelerators in the Universe. However, where and how these particles produce high-energy radiation is still an open question. What are the particle acceleration processes, which type of particles are the primary energy drivers, and what are the high-energy interaction processes are key puzzles in high-energy astrophysics? 


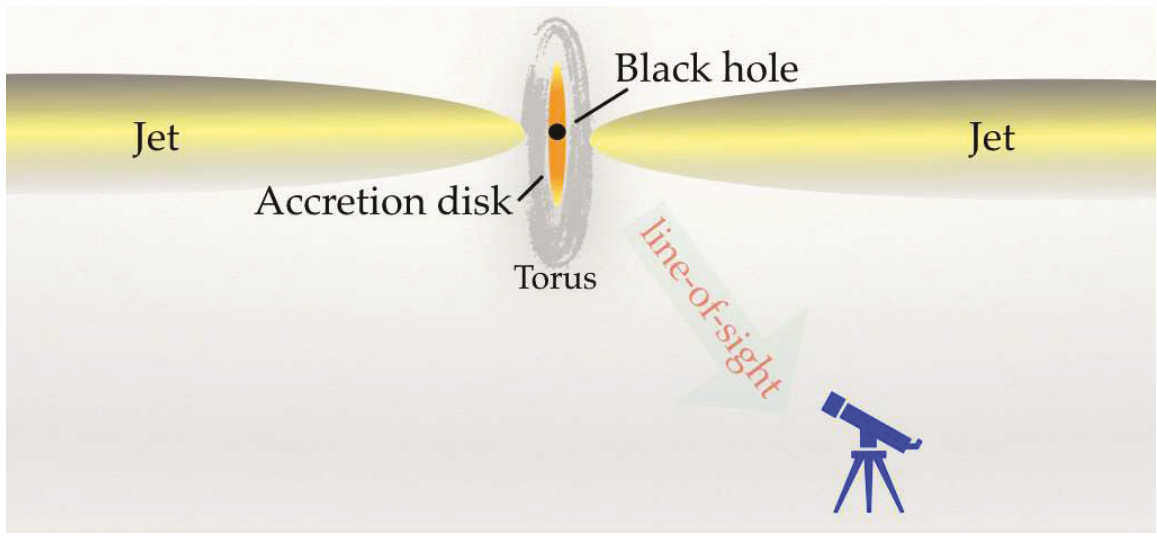

Figure 1. Sketch representing the mis-aligned radio emitting bipolar jets of a radio galaxy (not to scale). Jets typically extend up to a few hundreds of kilo-parsec to mega-parsec scales.

AGN having their jets pointed along our line-of-sight, called blazars, are the majority of the sources detected by the high-energy ground- and space-based telescopes. This goes along with the substantial Doppler boosting of their intrinsic emission. The radiation is beamed in our direction making these sources appear to be extremely bright and detectable even at larger redshifts. Blazars come in two flavors: BL Lacertae objects (BL Lacs) and flat spectrum radio quasars (FSRQs). In spite of the dissimilarity of their optical spectra-FSRQs show strong broad emission lines, while BL Lacs have only weak or no emission lines in their optical spectra-they share the same peculiar continuum properties (strong variability and high polarization). Most of the radiation we observe from these blazars is dominated by emission from the jet; however, characteristic signatures of emission from the immediate vicinity of the central black hole (accretion disk or corona) have also been observed, especially in FSRQs.

The radio to optical emission (and X-rays in some cases) from blazars is mostly synchrotron radiation from the jet, produced by relativistic electrons interacting with the jet's magnetic field. High-energy emission, which often dominates the spectral energy distribution (SED), is from inverse-Compton scattering of thermal photons (from the accretion disk, the broad-line region, or the molecular torus) and/or non-thermal photons (synchrotron emission from the jet) by the jet's relativistic electrons in case of leptonic models [1,9-12]. Gamma-ray emission could also be produced via photo-hadron interactions or hadron-nucleon (or proton-proton) collisions and subsequent cascades in the framework of hadronic models [9,12-14]. Detailed investigations of multi-wavelength flux and spectral variability of individual sources including cross-band (radio, optical, $X$-ray, $\gamma$-ray, and polarization), relative timing analysis of outbursts, and/or very long baseline interferometry (VLBI) component ejection/kinematics suggest that high-energy emission, especially $\gamma$ rays, are associated with the compact regions of relativistic jets energized by the central SMBHs (e.g., [1,2,6,15-22]). However, many critical details of particle acceleration mechanisms and radiation processes are still unknown.

Misaligned or non-blazar AGN, such as radio galaxies or Narrow line Seyfert 1 galaxies, although representing a small fraction $(<2 \%$, [23]) of the $\gamma$-ray detected sources, have emerged as an interesting class of $\gamma$-ray emitting AGN. Radio galaxies are a particularly interesting class of objects to understand several aspects of AGN physics and high-energy emission, i.e., how the relativistic outflows are launched and driven, what drives the particle acceleration, and how and where high-energy emission is produced in jets. Several of these key questions can be addressed via studying the TeVRad. This review paper summarizes the key observations of TeVRad, with an overview about the possible particle acceleration processes and emission mechanisms, and concentrates on the jet structure and 
multi-wavelength variability rather than spectral modeling, which is a separate topic. The interested reader is referred to the review article by Rieger and Levinson [24].

\section{Observations of the TeV Emitting Radio Galaxies}

Among the more than 30 radio galaxies observed by the Fermi/Large Area Telescope (LAT) reported in the LAT 8-year Point Source List (FL8Y: https: / fermi.gsfc.nasa.gov/ssc/data/access/lat/ $\mathrm{fl} 8 \mathrm{y} /$ ), six have also been detected at TeV energies (see Table 1). Most of these sources are relatively nearby; the closest is Centaurus A at a distance of 3.7 mega-parsec, while PKS 0625-35 (The source class for PKS 0625-35 is still under debate. The large-scale morphology of the source looks like that of radio galaxies, while its optical spectrum indicates a BL Lac classification [25].) at 220 mega-parsec distance is the farthest TeV-detected radio galaxy. The TeVRad, M87 and PKS 0625-35 have a black hole mass of a few $10^{9} \mathrm{M}_{\odot}$, which is similar to the average black hole mass of beamed radio galaxies a.k.a. blazars [26]. The other TeVRad host a smaller black hole, $\left(\mathrm{M}_{B H}=\mathrm{a}\right.$ few $\left.\times 10^{8} \mathrm{M}_{\odot}\right)$. The radio morphology of these sources resemble blazars when viewed at larger angles. The beaming effects are fairly moderate in TeVRad (apparent speed less than a few times the speed of light [27-29]).

Table 1. Radio galaxies detected at $\mathrm{TeV}$ energies.

\begin{tabular}{llccc}
\hline Source & Type & Redshift (Distance in Mpc) & $\mathbf{M}_{\boldsymbol{B H}}\left(\mathbf{M}_{\odot}\right)$ & $\mathbf{L}_{V H E}$ (erg s $^{-\mathbf{1}}$ ) \\
\hline Centaurus A & FR1 & $0.00183(3.7)[30]$ & $5 \times 10^{7}[31]$ & $10^{40}$ \\
M87 & FR1 & $0.0044(16)[32]$ & $6 \times 10^{9}[33]$ & $10^{41}$ \\
3C 84 & FR1 & $0.0177(71)[34]$ & $(3-8) \times 10^{8}[35,36]$ & $10^{45}$ \\
IC 310 & FR1 & $0.0189(80)[37]$ & $(1-7) \times 10^{8}[31,38]$ & $10^{44}$ \\
3C 264 & FR1 & $0.0217(95)[39]$ & $2.6 \times 10^{8}[40]$ & $6 \times 10^{43}$ \\
PKS 0625-35 & FR1/BL Lac & $0.05488(220)[41]$ & $3 \times 10^{9}[31]$ & $5 \times 10^{41}$ \\
\hline
\end{tabular}

The detection of the nearest TeVRad, Centaurus A core, at TeV energies was first reported by H.E.S.S. (High Energy Stereoscopic System) using more than $100 \mathrm{~h}$ of observations taken during 2004-2006 [42]. The source has been later detected also at GeV energies by the Fermi/LAT [43]. Fermi/LAT also discovered the spatial extension in the source, detecting $\gamma$-rays from both the core and from the extended giant lobes [43]. The combined GeV-TeV spectrum of the source shows a clear excess at $\mathrm{TeV}$ energies and cannot be fitted via a single zone emission model [44]. Moreover, the origin of the extended $\gamma$-ray emission still remains an open question, although several models were proposed, i.e., inverse-Compton scattering of relic radiation from the cosmic microwave background and the infrared-to-optical extragalactic background light [43].

At a distance of $\sim 16 \mathrm{Mpc}$, M87 was the first TeV detected radio galaxy [45]. M87 is the brightest galaxy in the Virgo cluster. The source has been detected on several occasions both in flaring and quiescent states [46-48]. The fastest variability timescale observed in the source is $\sim 1$ day [48]. Long-term variability on a few week timescales has also been reported for the source especially during quiescent states [47,49].

The Fermi telescope has detected 3C 84 since the beginning of its operation [50]. Fermi observations suggest that the $\mathrm{GeV}$ photon flux of the source has been continuously rising since then (see Section 4 for details). In 2009, the source was also detected at TeV energies [51,52]. In the end of 2016 and early 2017, the source has been through a dramatic flaring activity. During this epoch, the MAGIC telescopes detected the brightest flare ever observed in the source $\left(L_{\gamma-\text { ray }} \sim 10^{45} \mathrm{erg} \mathrm{s}^{-1}\right.$, [4] $)$ with a flux doubling timescale of $\sim 10 \mathrm{~h}$.

The radio galaxy IC 310 was first detected at TeV energies in 2009-2010 by the MAGIC telescope [53]. Variations on days to months timescales were observed in the source during this period. On 12-13 November 2012, a dramatic flare was observed in the source. During this exceptional variability phase, the observed flux doubling timescale of the observed $\mathrm{TeV}$ flares was as short as $5 \mathrm{~min}$. This is the fastest variability timescale observed in a radio galaxy up to now [38]. This extreme 
flare is quite challenging to be explained within the framework of any existing theoretical mechanism (see Section 6 for details).

At a distance of $95 \mathrm{Mpc}$, 3C 264 is rather a new member in the family. Nearly $12 \mathrm{~h}$ of VERITAS observations of the source between 9 February 2018 and 16 March 2018 confirms its detection at $\sim 5 \sigma$ level [54]. TeV emission above $250 \mathrm{GeV}$ from PKS 0625-35 was first reported in 2012 by H.E.S.S. [55]. Fermi observations of the source report variations on monthly timescales [56]. However, no variation has been seen at $\mathrm{TeV}$ energies. The source belongs to the category of low excitation line radio-loud AGN [57]. Radio observations find superluminal motion in the jet with apparent speeds up to $\sim 3 \mathrm{c}$ [58]. The evidence of a one-sided jet and superluminal motion suggests the source to be more like a blazar.

\section{Radio Properties}

\subsection{Parent Population}

All TeVRad are Fanaroff and Riley type I (FR1) sources. Moreover, most of the TeV-detected blazars (high-frequency peaked BL Lacs, HBL, in TeVcat: http://tevcat.uchicago.edu/) belong to the parent population of FR1 sources. Radio galaxies are classified as FR1 and FR2 [59] sources on the basis of their jet luminosity at $178 \mathrm{MHz}$ radio frequency. It was found that the sources with luminosity $\geq 5 \times 10^{24} \mathrm{~W} \mathrm{~Hz}^{-1} \mathrm{Sr}^{-1}$ belong to the FR2 class, while the rest were classified as FR1 type [59]. The key difference between the two populations is their radio morphology. FR2s are more powerful radio galaxies, and their radio morphology is characterized by powerful edge-brightened double lobes with prominent hot spots, while the FR1s are lower-power sources, which exhibit rather diffuse edge-darkened lobes. Moreover, FR2s tend to be found in less dense environments compared to FR1s. When seen at small inclinations, FR1 sources are BL Lacs while FR2s are radio-loud quasars (an excellent discussion about AGN unification can be found in Urry and Padovani [60]).

It was found that at a given radio power, FR1s have fainter nuclear X-ray and ultra-violet (UV) emission [61,62] compared to FR2s, which indicates that FR1s might have fainter accretion disks. For instance, the accretion rate for the brightest TeVRad 3C $84\left(L_{V H E}=10^{45} \mathrm{erg} \mathrm{s}^{-1}\right)$ is well below $10 \%$ of its Eddington accretion rate (Eddington ratio $\sim 3 \times 10^{-4},[63]$ ). The key question to answer is if this corresponds to different accretion modes in the two classes of sources [64]. If yes, it would be interesting to assess how it correlates to their observed TeV luminosity. Moreover, FR2s may occupy less dense environments, enabling lower $\gamma \gamma$-pair production opacity for the TeV photons. In dense environments, high-energy photons might get absorbed via a process called pair production when they interact with lower-energy (optical/UV) photons by producing electron-positron pairs.

\subsection{Jet Kinematics-Speeds on Parsec to Kilo-Parsec Scales}

The TeVRad M87 is the best studied radio galaxy because of its extremely bright and spectacular jet extending up to several hundreds of kilo-parsecs. Figure 2 depicts the kilo-parsec scale radio, optical, and X-ray images (from top to bottom) of the M87 jet. The high-resolution VLBI observations show very sub-luminal speeds $(\sim 0.01 \mathrm{c})$ on tens of parsec scales $[28,65,66]$, although recent higher-cadence VLBI monitoring programs have started to find faster motions at the similar scales [67,68]. Later, there is a sudden increase in the apparent speeds at a distance of $\sim 100$ parsecs close to the location of HST-1 feature (see Figure 3). Superluminal components with speeds up to $6 \mathrm{c}$ are detected after that (see Figure 3), which is followed by a smooth deceleration [69-71]. A similar velocity pattern has observed in 3C 264. The sub-luminal apparent speeds $(\leq 0.5 c)$ seemingly receive a kick at a distance of $\sim 100$ parsec where the jet has a stationary knot/feature. On kilo-parsec scales apparent speeds up to $7 c$ have been observed in the source [29]. Downstream acceleration with a change in speeds from $0.1-0.3$ to $0.5 c$ has also been observed in the kilo-parsec scale jet of Centaurus A [72-74]. How exactly these velocity fields develop and evolve along the jet is still an open issue. The stationary features are thought to be the sites of recollimation shocks, which might have a key role in accelerating the sub-luminal motion to super-luminal speeds. 

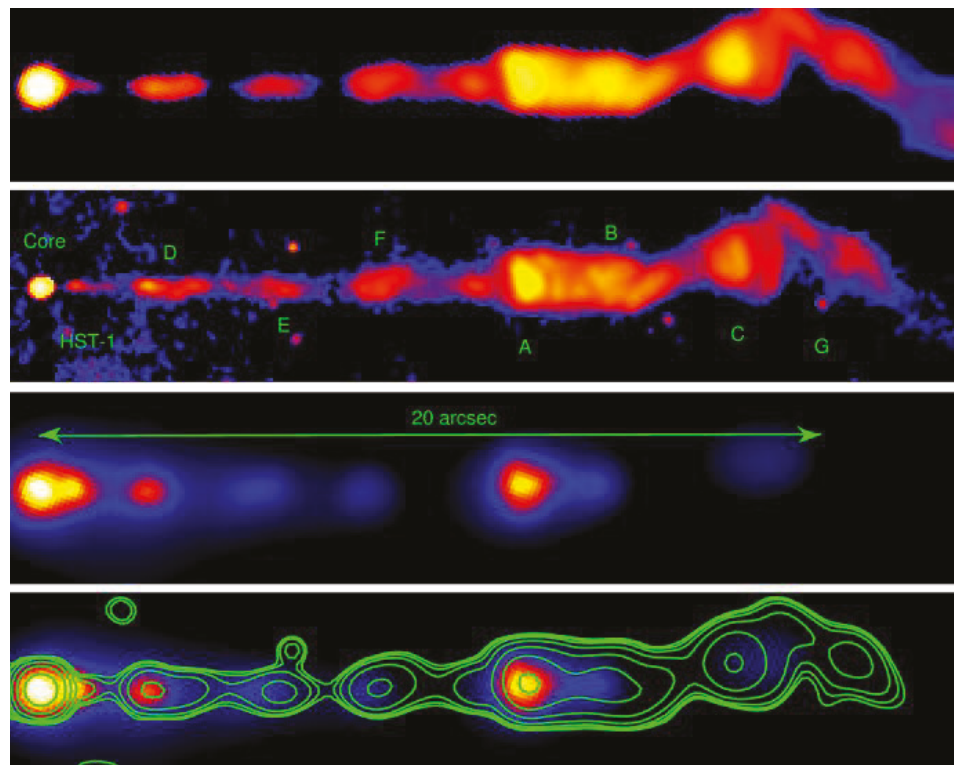

Figure 2. Multi-wavelength view of the M87 jet. From top to bottom: radio jet imaged by Very Large Array (VLA), optical jet seen by the Hubble Space Telescope, and X-ray image taken by the Chandra X-ray Observatory. The optical image (middle) marks the position of various bright knots/stationary features observed in the jet. (Credit: Marshall et al. [75], reproduced with permission (CAAS).

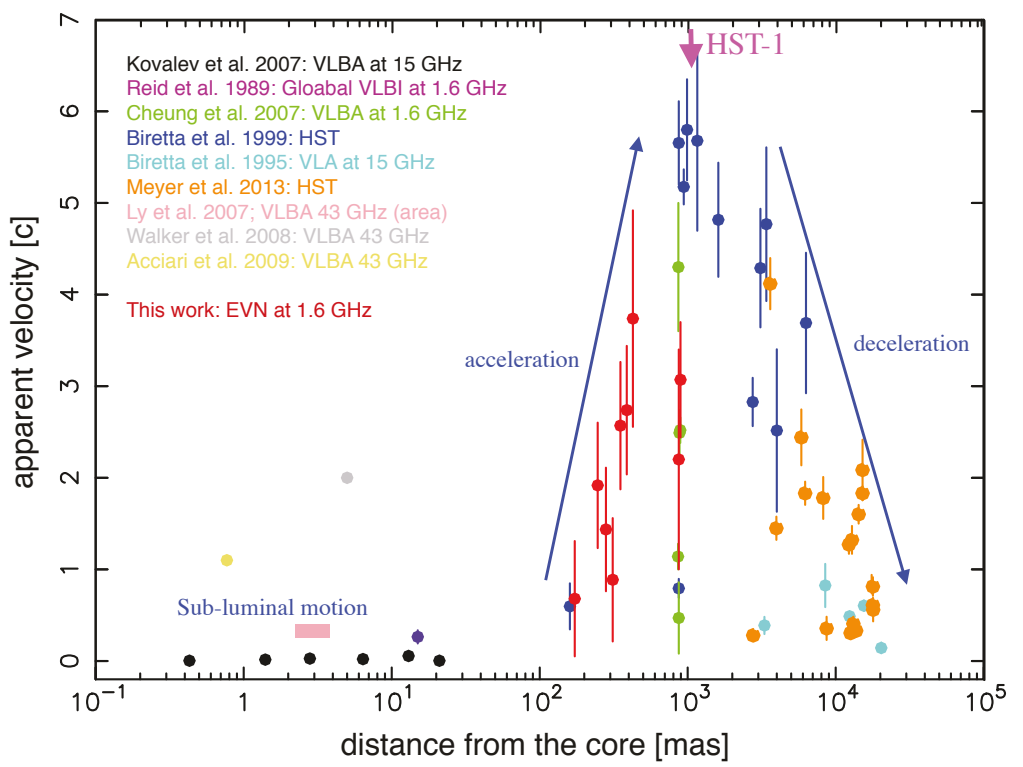

Figure 3. Velocity field measurements in the jet of M87 from parsec to kilo-parsec scales. The stationary feature HST- 1 is at a distance of $\sim 100$ parsecs from the central engine. The highest speeds are measured close to the position of HST-1. (Credit: Asada et al. [65], reproduced with permission (C)AAS). 
There has been no dramatic change noticed in the apparent speed of the moving features in the other TeVRad as a function of distance from the central engine. For instance, the observed speeds on parsec and kilo-parsec scales are sub-luminal $(\sim 0.2-0.9 c)$ in 3C 84 [8,76-78], suggesting a viewing angle $\geq 45^{\circ}$. The one-sided blazar like jet of IC 310 constrains the viewing angle to be $\leq 38^{\circ}$ implying a moderate beaming [79]. VLBI observations measure an apparent speed of $\sim 0.9-3 c$ in the PKS 0625-35 jet, which constrains the viewing angle in the source to be $\leq 13^{\circ}$ (Angioni et al. 2018, submitted).

Structured and stratified velocity patterns have also been observed in the parsec-scale jets of some of the TeVRad. For instance, a study by Mertens et al. [28] reports a stratification in the M87 jet with three different velocity patterns. The jet flow in the source has a slow, mildly relativistic layer, sheath, (apparent velocity $\sim 0.5 c$ ) and a fast spine (apparent velocity $\sim 0.9 c$ ). The study also discovered a significant difference in the apparent speeds observed in the northern $(0.5 c)$ and southern $(0.2 c)$ limbs of the jet (the bright limbs in the source can be seen in Figure 4). Likewise, limb brightening was discovered in the 3 C 84 jet using the space-VLBI observations [80]. Hints of differential motion were also observed in Centaurus A [72].

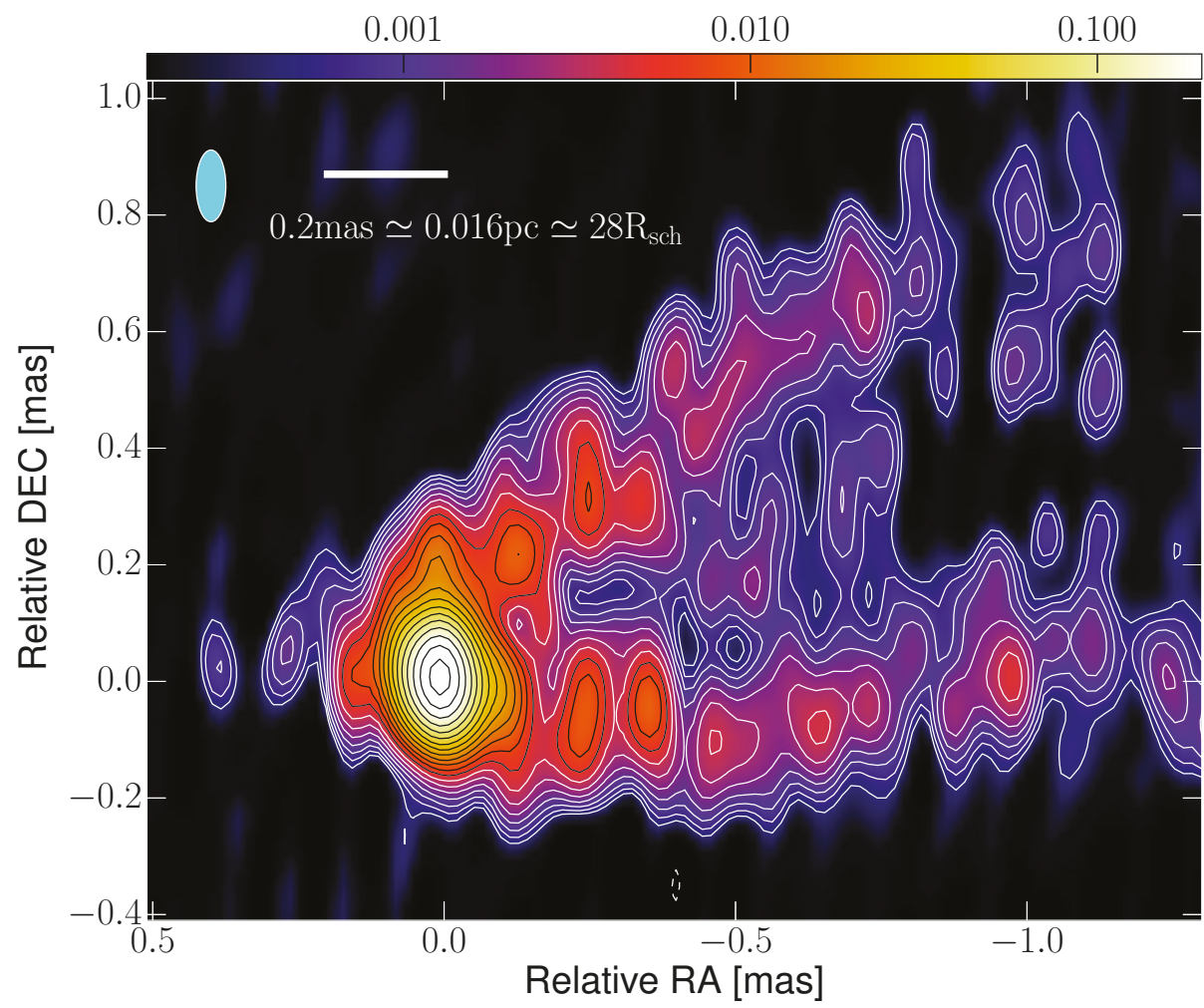

Figure 4. Stacked high-resolution radio image of M87 at $86 \mathrm{GHz}$ frequency (Credit: Kim et al. [81], reproduced with permission (C)ESO). The high-resolution radio imaging technique allows a zoom up to a scale of $\leq 10$ gravitational radii in the source frame.

\subsection{Zooming into the Event Horizon Scales}

AGN jets are the most spectacular structures in the Universe and represent the "extreme" and "largest" members of a wide family of jet-powered phenomena, including $\gamma$-ray bursts (GRBs), X-ray binaries (XRBs), and young stellar objects (YSOs). Over the past few decades, there has 
been remarkable progress in our understanding of AGN jet physics especially because of the development of high-resolution VLBI (for more details refer to the recent review on millimeter-VLBI by Boccardi et al. [82]). Millimeter-VLBI observations offer an angular resolution of a few tens of micro-arcseconds, which allows a probe of the inner jet region on scales $\leq 50$ gravitational radii $\left(R_{g}\right)$.

Given its proximity and fairly bright jet at millimeter radio frequencies, M87 is the best studied source using high-resolution VLBI $[28,65,81,83,84]$. Global millimeter VLBI observations at $86 \mathrm{GHz}$ radio frequency resolve a limb-brightened outflow down to $\sim 15 R_{g}$ [81,85]. A high-resolution image of M87 taken at $86 \mathrm{GHz}$ radio frequency is illustrated in Figure 4. The Event Horizon Telescope (EHT) observations at $230 \mathrm{GHz}$ and $345 \mathrm{GHz}$ frequencies constrain the size of the event horizon of the central black hole to be less than $10 R_{g}[84,86]$. These high-resolution images provided a unique tool to test the different jet-launching models $[83,84]$.

Another interesting study is made using the space-VLBI observations. The space-VLBI observations of $3 \mathrm{C} 84$ at $22 \mathrm{GHz}$ radio frequency using the RadioAstron mission probed the inner jet profile up to $100 \mathrm{R}_{g}$ [80]. The study discovered a very broad jet base with a transverse radius larger than about $250 R_{g}$ implying the jet to be more likely launched from the accretion disk. More interestingly, Global millimeter VLBI Array (GMVA) observations at $86 \mathrm{GHz}$ radio frequency discovered a double-nuclear structure at the jet base (Oh et al. 2018, in preparation).

High-resolution VLBI observations therefore offer an unparalleled opportunity to probe and pinpoint the high-energy emitting sites in the immediate vicinity of the central black hole. Moreover, high-resolution polarization imaging is a unique technique to unravel the magnetic field topology on scales less than a few hundreds of gravitational radii. The tiny magnetized regions in the extended jets are the potential sites of high-energy emission $[20,87,88]$.

\section{TeV Variability}

Like blazars, $\mathrm{TeV}$ Rad exhibit variability on multiple timescales i.e., sub-hour to days timescale flares are accompanied by long-term outbursts. For instance, multiple modes of flaring activity can be seen in the $\gamma$-ray light curve of 3C 84 observed by the Fermi/LAT. Figure 5 presents the rapid flares superimposed on top of the long-term rising trend in the Fermi photon flux light curve (in blue). At the end of 2016, several extremely bright and rapid flares were observed by Fermi/LAT. The fastest flares seen by Fermi /LAT have flux doubling timescale of 9-12 h. In 2016-2017, the source was also detected multiple times at TeV energies by the MAGIC telescope (see Figure 6) [4]. During the course of this period, MAGIC detected the fastest and most luminous flare from 3C 84. An extremely bright $\left(L_{\gamma} \simeq 10^{45} \mathrm{erg} \mathrm{s}^{-1}\right)$ flare with a flux doubling timescale of $\sim 10 \mathrm{~h}$ was observed on 1 January 2017 [4]. Flux variations on multiple timescales were also observed in IC 310 and M87. 


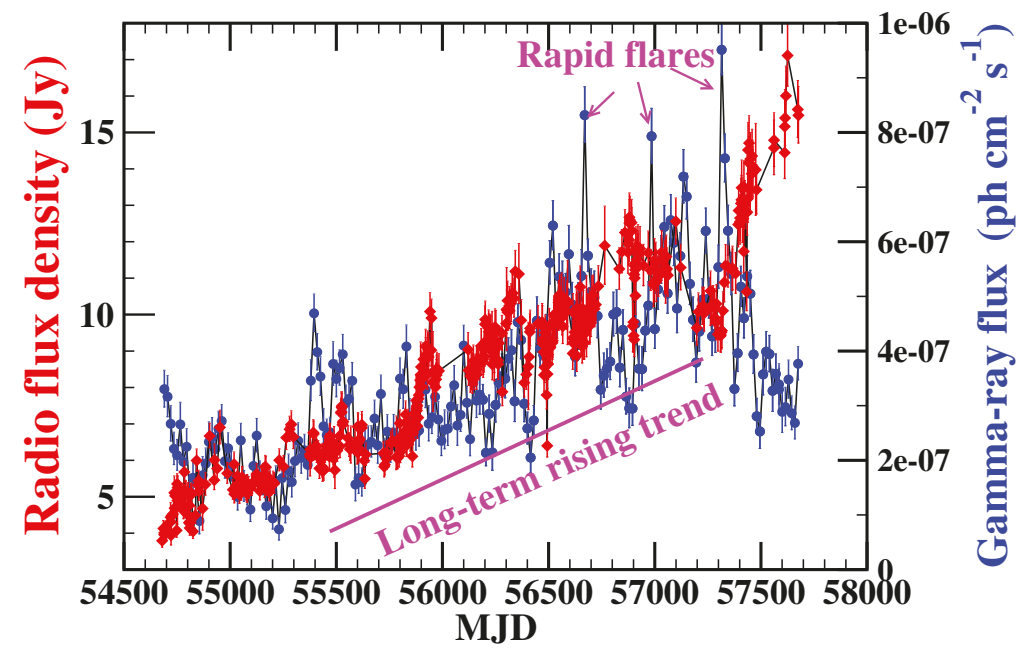

Figure 5. Gamma-ray photon flux (blue) and radio $230 \mathrm{GHz}$ (red) light curve of the TeVRad $3 \mathrm{C} 84$ at E $=0.1-300 \mathrm{GeV}$. The $\gamma$-ray light curve is produced by adaptive binning analysis method following Lott et al. [89] using the Fermi/LAT data from August 2008 until September 2017. The radio $230 \mathrm{GHz}$ light curve is from sub-millimeter array (SMA) AGN monitoring program (details are refereed to Hodgson et al. [8]). Rapid flares superimposed on top of the long-term rising trend can be seen both at $\gamma$-ray and radio frequencies.

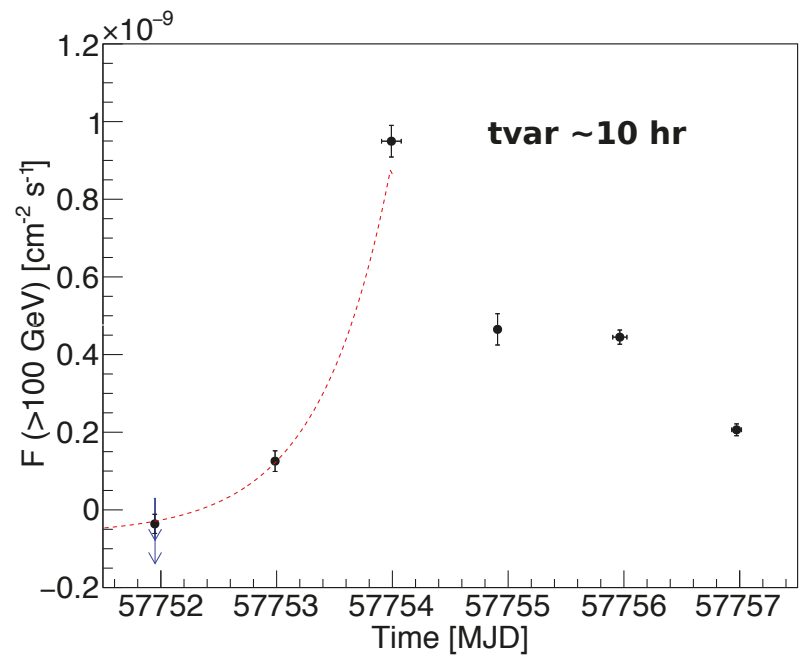

Figure 6. TeV light curve of 3C 84 observed by the MAGIC telescope [4]. The fastest flare observed around January 01,2017 has a flux doubling timescale of $\sim 10 \mathrm{~h}$. Long-term variations at $\gamma$-rays are shown in Figure 5.

Extremely short variability timescales in TeVRad have attracted the attention of astrophysicists, as the observed timescales can reach down to the light crossing time of the black hole event horizon, $t_{B H}=r_{g} / c$, where $r_{g}$ is the gravitational radius of the black hole and $c$ is the speed of light. For instance, the observed variability timescale $\left(t_{v a r}=5 \mathrm{~min}\right)$ in IC 310 is much smaller than the event horizon light crossing timescale, $t_{B H}=25 \mathrm{~min}$ [38]. The fastest variability timescale observed in M87 $\left(\mathrm{t}_{\text {var }} \sim\right.$ 
1 day) is comparable with the event horizon light crossing timescale ( $\left.t_{B H} \sim 60-90 \mathrm{~min}\right)$. Variations on sub-horizon scales have also been observed in blazars [90]. However, in case of blazars, one can always argue that beaming effects make the observed timescale appear shorter. This requires the Doppler factor to be greater than 50 , which has rarely been observed [27,78].

Understanding the physical processes responsible for the origin of the ultra-fast $\mathrm{TeV}$ flares is a key challenge in high-energy astrophysics. Sub-horizon scale variations and extremely luminous flares, especially in the absence of strong beaming effects, require (i) emission regions of extremely high electromagnetic energy of the emitting particles, (ii) efficient particle acceleration mechanisms which could channel the electromagnetic energy to particle energy, and (iii) less-dense photon environments so that the emitted $\mathrm{TeV}$ photons do not get self-absorbed by $\gamma \gamma$-pair production.

\section{High-Energy Emitting Sites}

Locating the $\gamma$-ray emitting sites is a key challenge not only in case of radio galaxies but also for blazars. When the emission region is closer to the central black hole, there is higher proportion of electromagnetic energy available in the jet, making it easy to account for the observed extremely bright $\gamma$-ray flashes. However, if the high-energy photons are produced too close to the black hole, the chances for them to escape through the rich photon environments are low. Nevertheless, at least some of these high-energy photons seem to survive even through dense environments. Photons produced further down in the jet have higher chances of not being absorbed; however, accelerating particles to extreme high-energy is a quite challenge there (see Section 6 for more details).

The sub-horizon scale emitting regions, detected in IC 310, 3C 84, and M87, could either be very close to the black hole or further down in the jet. The TeV flare in IC 310 was interpreted as originating from the magnetospheric gap of the black hole (see Section 6) as it is hard to achieve the high luminosity and rapid variability further down in the jet. The rapid TeV flare in $3 \mathrm{C} 84$ was also proposed to be produced in the magnetospheric gap; however, achieving the extremely high luminosity requires an extremely fast rotating black hole and magnetic field higher than its equipartition value [4]. However, these studies lack a conclusive observational evidence for the emission to be produced close to the black hole. Multi-frequency observations of M87 during an episode of extremely rapid flares provided an opportunity to study the $\mathrm{TeV}$ emitting regions in great detail. Strong $\mathrm{TeV}$ flares in the source were accompanied by an increase of the flux from the nucleus observed at $43 \mathrm{GHz}$ radio frequency [5], suggesting the emission region to be located within the radio core ( $\leq 50$ gravitational radii). The extreme compactness of the $\mathrm{TeV}$ emission region and the observed $\mathrm{TeV}$-radio connection in M87 conclusively infer that the VHE emission is indeed produced very close to the black hole [5]. This scenario has further been strengthened by more recent TeV-VLBI joint observations of M87 [91,92], where the authors also found the flux increase of the $43 \mathrm{GHz}$ core along with $\mathrm{TeV}$ flaring events.

Hints of multiple $\gamma$-ray emitting regions have also been observed in TeV Rad. For instance, a study performed using the KVN (Korean VLBI Network) and $\gamma$-ray observations indicate the presence of multiple $\gamma$-ray emitting regions in $3 C 84$ [8]. Figure 5 presents a comparison of the flux variations observed at $\gamma$-ray and $230 \mathrm{GHz}$ radio frequencies. The long-term rising trend is present in the flux variations seen both at radio and $\gamma$-ray frequencies. However, the rapid flares do not have a one-to-one correspondence at the two frequencies. A formal cross-correlation analysis indicates two prominent peaks at around -500 and +400 days, which implies that $\gamma$-rays variations lead those at radio by -500 days and also lag behind by +400 days (see Figure 7 ). A detailed investigation of the flux density variations in the spatially separated emission regions of the jet indicates the presence of multiple $\gamma$-ray emitting sites in the source. The study reported that there are two active regions producing $\gamma$-rays in the source, one very close to the black hole and another at a distance of $\sim 4$ parsec in the source frame. 


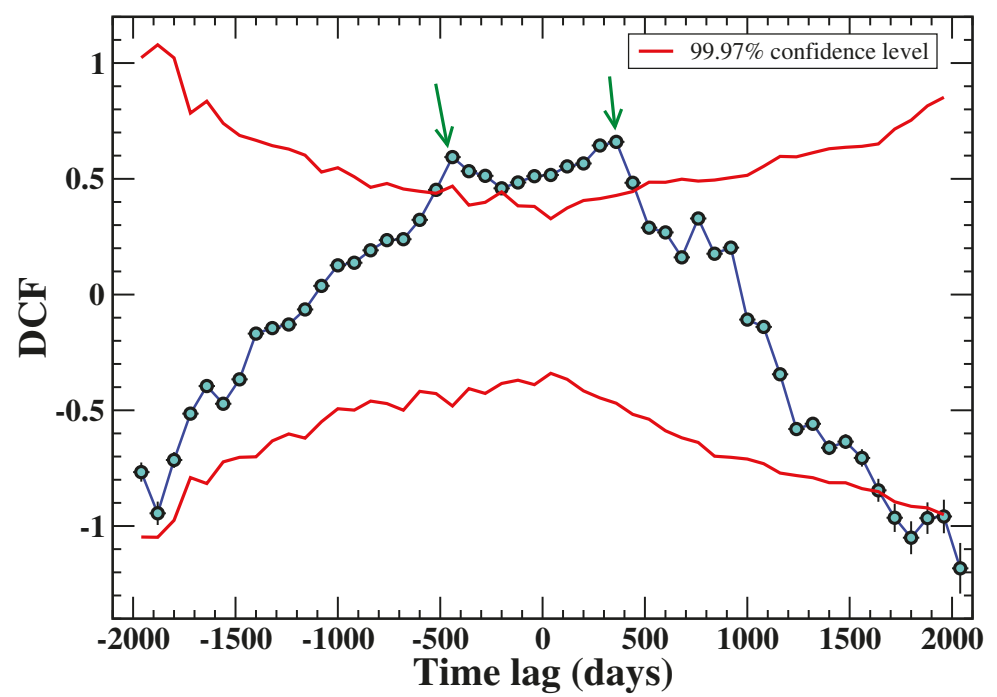

Figure 7. Cross-correlation analysis curve of the $\gamma$-ray and radio flux variations observed in 3C 84 (see Figure 5). The two peaks at -500 and +400 days (green arrows) imply $\gamma$-rays flux variations leading and lagging those at radio. A detailed analysis suggests the presence of multiple $\gamma$-ray emitting sites in the source [8].

Fermi has detected $\gamma$-rays both from the core and the extended lobes of the nearest TeVRad Centaurus A [43]. The source has a spatial extension of about 9 degrees across the sky, which corresponds to $\sim 600$ kilo-parsec, and the $\gamma$-ray morphology (Magill et al. 2019, in preparation) is quite similar to its radio jet morphology, which implies $\gamma$-rays coming from both the central AGN core and extended kilo-parsec scale lobes. Given the detection of extended $\gamma$-ray emission at GeV energies and the absence of $\mathrm{TeV}$ variability, the extension of $\mathrm{TeV}$ emission cannot be discarded. It is important to note that Fermi/LAT has detected extended $\mathrm{GeV}$ emission also from Fornax A. It might be interesting to see for how many of these sources spatial extension can be detected by using the future Cherenkov Telescope Array.

\section{Particle Acceleration Mechanisms}

The observed extremely rapid variability and enormously high $\gamma$-ray luminosity are clearly challenging to achieve in astrophysical jets as they require (i) particle acceleration to extreme high energies in the presence of strong radiative loss (ii) variation on sub-horizon timescales, and (iii) conversion of large volumes of electromagnetic energy with enormous efficiency. Several mechanisms have been proposed to address the $\mathrm{TeV}$ challenge in astrophysical sources. Some recent ideas are summarized below.

The most common mechanism is relativistic shock i.e., the flow is accelerated to supersonic speeds and strong shocks are formed in the relativistic outflow because of (i) faster moving outflow runs into slower flow resulting in the formation of internal shock, (ii) the outflow encounters an obstacle (perhaps a molecular cloud) on its way, (iii) any other perturbation happening at the base of the jet could also form a shock wave to form and propagate down the jet. Relativistic shocks [93] seem to be effective in low-magnetization regimes and could explain the observed long-term variability from AGN fairly well $[1,20,87,88,94-96]$. However, the luminous ultra-fast TeV flares observed from a number of AGN $[4,38,90,97]$ are difficult to explain via the relativistic shock model, especially in the absence of substantial Doppler beaming, and therefore challenge our understanding of particle acceleration processes. 
Magnetic reconnection, a process by which magnetic energy is transferred to particle heat, bulk energy and non-thermal energy, is proposed to be an effective mechanism for energy dissipation [98-102] in magnetically dominated relativistic outflows. During magnetic reconnection, opposite polarity magnetic field lines exchange partners at "X-points" leading to breaking of flux freezing and change in magnetic field topology, which results in an explosive release of energy. For instance, solar flares seem to be powered by magnetic reconnection. However, this picture is far too complex in case of relativistic outflows. Nevertheless, there has been great advancement in simulations to understand the relativistic magnetic reconnection. The particle-in-cell (PIC) magnetic reconnection simulations suggest that ultra-relativistic plasmoids generated by magnetic reconnection $[99,100]$ can power the ultra-fast flares observed from TeV AGN. An example $\gamma$-ray light curve (top) and snapshot of relativistic magnetic reconnection (bottom) by an integrated modeling relying on first-principle PIC kinetic simulation and an advanced polarized radiation transfer simulation from Zhang et al. [98] are illustrated in Figure 8. The study reports that plasmoid coalescences in the magnetic reconnection layer can produce highly variable multi-wavelength light curves, and also can explain polarization degree and polarization angle variations.
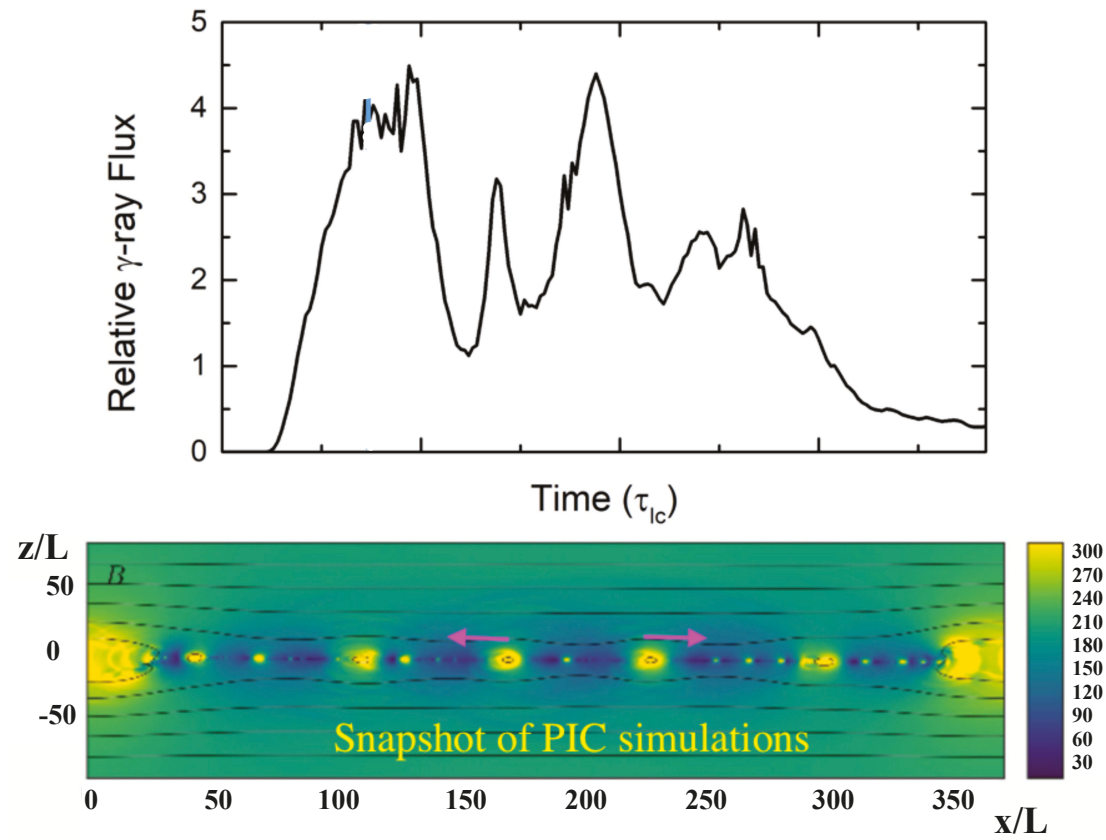

Figure 8. Radiative signatures of relativistic magnetic reconnection by an integrated modeling relying on first-principle particle-in-cell (PIC) kinetic simulation (Credit: Haocheng Zhang [98]). (Top) $\gamma$-ray light curve in units of light crossing time scale. (Bottom) reconnection layer in the unit of ion skin depth.

A magnetospheric gap model was invoked to explain the sub-horizon scale flux variations in $\mathrm{TeV}$ Rad $[4,38,103,104]$. It has been proposed that a rotating black hole embedded in a strong magnetic field (of the order of $10^{4}-10^{5} \mathrm{G}$ ) will induce an electric field, $E$, corresponding to a voltage drop across its event horizon, which facilitates an efficient electromagnetic extraction of the rotational energy of the black hole (for more details, see the review by Rieger [104]), and could be an efficient mechanism to power the TeV flares in AGN. The maximum extractable energy ( $L_{\text {magnetosphere }}$ ) from the black hole, however, depends on the details of the gap configuration. For instance, $L_{\text {magnetosphere }}$ scales as the height 
of the magnetospheric gap $(h)$ i.e., $L_{\text {magnetos phere }} \sim L_{B Z}\left(h / r_{g}\right)^{\beta}$, where $L_{B Z}$ is the maximum power from a fast rotating black hole [105] and $\beta$ depends on the gap setup. The size of the gap can be constrained by the observed $\mathrm{TeV}$ variations $\left(c . \delta t \leq r_{g}\right.$ ), which further reduces the maximum $L_{\text {magnetosphere }}$ (details are referred to $[104,106])$. Another important aspect of this model is the absorption of the emitted TeV photons by the thermal photon environment of the black hole. In order for the $\mathrm{TeV}$ photons to survive through the accretion disk environment, the magnetospheric gap model requires an under-luminous or radiatively inefficient accretion flow to avoid $\gamma \gamma$-pair production. This enforces accretion rates to be below $1 \%$ of the Eddington scale. Putting this in the context of current observations, the model could explain the observed TeV flares in M87 fairly well; however, the minute-scale TeV flare in IC 310 seems less likely to have a magnetospheric gap origin. The observed TeV luminosity in $3 \mathrm{C} 84$ is also hard to match in the framework of the magnetospheric gap model unless the magnetic field threading the accretion disk is a factor of 1000 greater than its equipartition value.

Magnetoluminescence $[107,108]$ has been recently proposed to explain the observed TeV flares from astrophysical sources. The basic idea is that rotating at the center of an AGN leads to writhing of magnetic flux ropes resulting in tangled and knotted magnetic field lines. The tightly wound magnetic flux ropes especially in the inner jet are highly dynamically unstable. The tangled/knotted flux lines when opened up or untangled could produce rapid $\gamma$-ray flares. It has been suggested that this phenomenon includes an implosion rather than explosion [108]. As a consequence, particles could be accelerated to significantly higher energies as the dissipating and cooling volume could be re-energized by the medium external to it. Electromagnetic detonation [108] is proposed as an alternative possibility, which might accelerate particles at the transition of high-magnetized outflow into low-magnetized outflow and energy-flux. Particles are subjected to unbalanced electric field at the transition and get accelerated (positive and negative charged particles are accelerated in opposite direction) to produced $\gamma$-rays. In comparison to magnetic reconnection, which requires a magnetic flux to channel into a small volume, electromagnetic detonation will have a larger volume for the conversion of electromagnetic energy into particle energy.

\section{Future Directions}

Pinpointing the sub-horizon scale $\gamma$-ray emitting sites in kilo-parsec to mega-parsec scales AGN jets is a key challenge in high-energy astrophysics. A coordinated multi-wavelength and multi-messenger effort is needed to better understand it. The TeV emitting radio galaxies ( $\mathrm{TeVRad}$ ) are a particularly interesting class of AGN to unravel the mystery of the origin of $\gamma$-ray emission. Some important considerations are:

- $\quad \mathrm{TeVRad}$ are nearby AGN. The farthest detected TeVRad is at a distance of $220 \mathrm{Mpc}$. Using the millimeter-VLBI observations, we can probe regions in the immediate vicinity of the central black hole i.e., down to a scale of less than 50 gravitational radii in the source frame (for more details about the imaging capabilities of the current and near-future high-resolution VLBI observations, check Boccardi et al. [82]). The mm-VLBI observations will provide an ultimate opportunity to probe the compact regions closer to the black hole, which are the potential sites of high-energy emission. High-resolution polarization imaging will probe the magnetic field strength and configuration of these compact emission regions, which is an essential piece of information in order to understand the production mechanisms of high-energy emission.

- $\quad$ Being seen off-axis, TeVRad offers a unique opportunity to transversely resolve the jet. Using high-resolution VLBI, one can transversely resolve the fine scale jet structure and can probe their flux density and polarization variations. VLBI astrometry observations could provide the exact location of the core. Having that, we could combine the high-resolution VLBI imaging and multi-wavelength observations to exactly pinpoint the location of $\gamma$-ray emitting sites.

- There have been great advances in the theory and simulation front. Several details about plasma physics under extreme conditions can be better understood via simulations i.e., radiative signatures of relativistic magnetic reconnection [98-102], formation of jets and expulsion of 
magnetic flux from the central black hole [109-111], high-energy polarization signatures of shocks and reconnection $[98,112,113]$. A comparison of predictions from simulations with measurements will be crucial to understanding the physical processes happening around black holes.

- Cherenkov Telescope Array (CTA) [114] with its sensitivity better than the existing GeV/TeV instruments and spatial resolution of the order of a couple of arc-minutes at $\mathrm{TeV}$ energies will provide observations up to $300 \mathrm{TeV}$. CTA will detect many more misaligned AGN and will also help us in separating nuclear and off-nuclear components especially for nearby sources. Having the potential to probe deeper into the spectral and variability characteristics of AGN, CTA will eventually revolutionize our understanding of the $\mathrm{TeV}$ sky.

- The question of what powers $\gamma$-ray AGN flares is ultimately related to the energy dissipation mechanism at work. High-energy polarization observations will deliver an ultimate test for probing the energetic particle-acceleration processes and emission mechanisms: (i) leptonic versus hadronic models and (ii) shocks versus magnetic reconnection. High-energy polarimetry missions are on their way to unravel how the most efficient particle accelerators in the Universe work. For instance, the All-sky Medium Energy Gamma-ray Observatory (AMEGO: https: / /asd. gsfc.nasa.gov/amego/) will offer $\mathrm{MeV}$ polarization observations of the $\gamma$-ray bright AGN. The Imaging X-ray Polarimetry Explorer (IXPE: https:/ / wwwastro.msfc.nasa.gov/ixpe/index.html) will observe polarization signatures from $X$-ray bright AGN.

- The recent observations of coincidence of a high-energy neutrino event with a flaring AGN, TXS 0506+056 indicate AGN as potential sources of high-energy neutrinos [115]. Multi-wavelength and multi-messenger observations of similar events will test if AGN jets are powerful sources of extra-galactic neutrinos and put constraints on lepto-hadronic models.

Given the flood of high quality data from the current and upcoming missions and great advances in theory/simulation, it is an exciting time for astronomers. There are several major discoveries to be made.

Acknowledgments: B.R. acknowledges the help of Dave Thompson, Chris Shrader, Lorentzo Sironi, Benoit Lott, Zorawar Wadiasingh, and Alice Harding for their fruitful discussions and comments that improved the manuscript.

Funding: This research was supported by an appointment to the NASA Postdoctoral Program at the Goddard Space Flight Center, administered by Universities Space Research Association through a contract with NASA.

Conflicts of Interest: The authors declare no conflicts of interest.

\section{References}

1. Rani, B.; Krichbaum, T.P.; Fuhrmann, L.; Böttcher, M.; Lott, B.; Aller, H.D.; Aller, M.F.; Angelakis, E.; Bach, U.; Bastieri, D.; et al. Radio to gamma-ray variability study of blazar S5 0716+714. Astron. Astrophys. 2013, 552, A11. [CrossRef]

2. Rani, B.; Krichbaum, T.P.; Marscher, A.P.; Jorstad, S.G.; Hodgson, J.A.; Fuhrmann, L.; Zensus, J.A. Jet outflow and gamma-ray emission correlations in S5 0716+714. Astron. Astrophys. 2014, 571, L2. [CrossRef]

3. Algaba, J.C.; Lee, S.S.; Kim, D.W.; Rani, B.; Hodgson, J.; Kino, M.; Trippe, S.; Park, J.H.; Zhao, G.Y.; Byun, D.Y.; et al. Exploring the Variability of the Flat Spectrum Radio Source 1633+382. I. Phenomenology of the Light Curves. Astrophys. J. 2018, 852, 30. [CrossRef]

4. MAGIC Collaboration; Ansoldi, S.; Antonelli, L.A.; Arcaro, C.; Baack, D.; Babić, A.; Banerjee, B.; Bangale, P.; Barres de Almeida, U.; Barrio, J.A.; et al. Gamma-ray flaring activity of NGC 1275 in 2016-2017 measured by MAGIC. Astron. Astrophys. 2018, 617, 91.

5. Acciari, V.A.; Aliu, E.; Arlen, T.; Bautista, M.; Beilicke, M.; Benbow, W.; Bradbury, S.M.; Buckley, J.H.; Bugaev, V.; Butt, Y.; et al. Radio Imaging of the Very-High-Energy $\gamma$-Ray Emission Region in the Central Engine of a Radio Galaxy. Science 2009, 325, 444. [PubMed]

6. Agudo, I.; Jorstad, S.G.; Marscher, A.P.; Larionov, V.M.; Gómez, J.L.; Lähteenmäki, A.; Gurwell, M.; Smith, P.S.; Wiesemeyer, H.; Thum, C.; et al. Location of $\gamma$-ray Flare Emission in the Jet of the BL Lacertae Object OJ287 More than 14 pc from the Central Engine. Astrophys. J. Lett. 2011, 726, L13. [CrossRef] 
7. Jorstad, S.G.; Marscher, A.P.; Smith, P.S.; Larionov, V.M.; Agudo, I.; Gurwell, M.; Wehrle, A.E.; Lähteenmäki, A.; Nikolashvili, M.G.; Schmidt, G.D.; et al. A Tight Connection between Gamma-Ray Outbursts and Parsec-scale Jet Activity in the Quasar 3C 454.3. Astrophys. J. 2013, 773, 147. [CrossRef]

8. Hodgson, J.A.; Rani, B.; Lee, S.S.; Algaba, J.C.; Kino, M.; Trippe, S.; Park, J.H.; Zhao, G.Y.; Byun, D.Y.; Kang, S.; et al. KVN observations reveal multiple $\gamma$-ray emission regions in 3C 84? Mon. Not. R. Astron. Soc. 2018, 475, 368-378. [CrossRef]

9. Böttcher, M.; Reimer, A.; Zhang, H. Leptonic and Hadronic Modeling of Fermi-Detected Blazars. Spectral Energy Distribution Modeling and High-Energy Polarization Predictions. Eur. Phys. J. Web Conf. 2013, 61, 05003. [CrossRef]

10. Böttcher, M. Modeling the emission processes in blazars. In The Multi-Messenger Approach to High-Energy Gamma-Ray Sources; Springer: Dordrecht, The Netherlands, 2007; pp. 95-104.

11. Rybicki, G.B.; Lightman, A.P. Radiative Processes in Astrophysics; Wiley: New York, NY, USA, 1986; p. 400.

12. Dermer, C.D.; Menon, G. High Energy Radiation from Black Holes: Gamma Rays, Cosmic Rays, and Neutrinos; Princeton Univerisity Press: Princeton, NJ, USA, 2009.

13. Zhang, H.; Böttcher, M. X-Ray and Gamma-Ray Polarization in Leptonic and Hadronic Jet Models of Blazars. Astrophys. J. 2013, 774, 18. [CrossRef]

14. De Angelis, A.; Mallamaci, M. Gamma-ray astrophysics. Eur. Phys. J. Plus 2018, 133, 324. [CrossRef]

15. Jorstad, S.G.; Marscher, A.P.; Mattox, J.R.; Aller, M.F.; Aller, H.D.; Wehrle, A.E.; Bloom, S.D. Multiepoch Very Long Baseline Array Observations of EGRET-detected Quasars and BL Lacertae Objects: Connection between Superluminal Ejections and Gamma-Ray Flares in Blazars. Astrophys. J. 2001, 556, 738-748. [CrossRef]

16. Jorstad, S.G.; Marscher, A.P.; Larionov, V.M.; Agudo, I.; Smith, P.S.; Gurwell, M.; Lähteenmäki, A.; Tornikoski, M.; Markowitz, A.; Arkharov, A.A.; et al. Flaring Behavior of the Quasar 3C 454.3 Across the Electromagnetic Spectrum. Astrophys. J. 2010, 715, 362-384. [CrossRef]

17. Marscher, A.P.; Jorstad, S.G.; Larionov, V.M.; Aller, M.F.; Aller, H.D.; Lähteenmäki, A.; Agudo, I.; Smith, P.S.; Gurwell, M.; Hagen-Thorn, V.A.; et al. Probing the Inner Jet of the Quasar PKS 1510-089 with Multi-Waveband Monitoring During Strong Gamma-Ray Activity. Astrophys. J. Lett. 2010, 710, L126-L131. [CrossRef]

18. Marscher, A.P.; Jorstad, S.G. The Megaparsec-scale X-ray Jet of The BL Lac Object OJ287. Astrophys. J. 2011, 729, 26. [CrossRef]

19. Schinzel, F.K.; Lobanov, A.P.; Taylor, G.B.; Jorstad, S.G.; Marscher, A.P.; Zensus, J.A. Relativistic outflow drives $\gamma$-ray emission in 3C 345. Astron. Astrophys. 2012, 537, A70. [CrossRef]

20. Rani, B.; Krichbaum, T.P.; Marscher, A.P.; Hodgson, J.A.; Fuhrmann, L.; Angelakis, E.; Britzen, S.; Zensus, J.A. Connection between inner jet kinematics and broadband flux variability in the BL Lacertae object S5 0716+714. Astron. Astrophys. 2015, 578, A123. [CrossRef]

21. Orienti, M.; Koyama, S.; D'Ammando, F.; Giroletti, M.; Kino, M.; Nagai, H.; Venturi, T.; Dallacasa, D.; Giovannini, G.; Angelakis, E.; et al. Radio and $\gamma$-ray follow-up of the exceptionally high-activity state of PKS 1510-089 in 2011. Mon. Not. R. Astron. Soc. 2013, 428, 2418-2429. [CrossRef]

22. Raiteri, C.M.; Villata, M.; D'Ammando, F.; Larionov, V.M.; Gurwell, M.A.; Mirzaqulov, D.O.; Smith, P.S.; Acosta-Pulido, J.A.; Agudo, I.; Arévalo, M.J.; et al. The awakening of BL Lacertae: observations by Fermi, Swift and the GASP-WEBT. Mon. Not. R. Astron. Soc. 2013, 436, 1530-1545. [CrossRef]

23. Ackermann, M.; Ajello, M.; Atwood, W.B.; Baldini, L.; Ballet, J.; Barbiellini, G.; Bastieri, D.; Becerra Gonzalez, J.; Bellazzini, R.; Bissaldi, E.; et al. The Third Catalog of Active Galactic Nuclei Detected by the Fermi Large Area Telescope. Astrophys. J. 2015, 810, 14. [CrossRef]

24. Rieger, F.M.; Levinson, A. Radio Galaxies at VHE energies. arXiv 2018. Available online: http:/ / xxx.lanl. gov/abs/1810.05409 (accessed on 22 January 2019).

25. Wills, K.A.; Morganti, R.; Tadhunter, C.N.; Robinson, T.G.; Villar-Martin, M. Emission lines and optical continuum in low-luminosity radio galaxies. Mon. Not. R. Astron. Soc. 2004, 347, 771-786. [CrossRef]

26. Falomo, R.; Carangelo, N.; Treves, A. Host galaxies and black hole masses of low- and high-luminosity radio-loud active nuclei. Mon. Not. R. Astron. Soc. 2003, 343, 505-511. [CrossRef]

27. Lister, M.L.; Aller, M.F.; Aller, H.D.; Homan, D.C.; Kellermann, K.I.; Kovalev, Y.Y.; Pushkarev, A.B.; Richards, J.L.; Ros, E.; Savolainen, T. MOJAVE: XIII. Parsec-scale AGN Jet Kinematics Analysis Based on 19 years of VLBA Observations at 15 GHz. Astron. J. 2016, 152, 12. [CrossRef] 
28. Mertens, F.; Lobanov, A.P.; Walker, R.C.; Hardee, P.E. Kinematics of the jet in M 87 on scales of 100-1000 Schwarzschild radii. Astron. Astrophys. 2016, 595, A54. [CrossRef]

29. Meyer, E.T.; Georganopoulos, M.; Sparks, W.B.; Perlman, E.; van der Marel, R.P.; Anderson, J.; Sohn, S.T.; Biretta, J.; Norman, C.; Chiaberge, M. A kiloparsec-scale internal shock collision in the jet of a nearby radio galaxy. Nature 2015, 521, 495-497. [CrossRef] [PubMed]

30. Harris, G.L.H.; Rejkuba, M.; Harris, W.E. The Distance to NGC 5128 (Centaurus A). Publ. Astron. Soc. Aust. 2010, 27, 457-462. [CrossRef]

31. Bettoni, D.; Falomo, R.; Fasano, G.; Govoni, F. The black hole mass of low redshift radiogalaxies. Astron. Astrophys. 2003, 399, 869-878. [CrossRef]

32. Bird, S.; Harris, W.E.; Blakeslee, J.P.; Flynn, C. The inner halo of M 87: A first direct view of the red-giant population. Astron. Astrophys. 2010, 524, A71. [CrossRef]

33. Walsh, J.L.; Barth, A.J.; Ho, L.C.; Sarzi, M. The M87 Black Hole Mass from Gas-dynamical Models of Space Telescope Imaging Spectrograph Observations. Astrophys. J. 2013, 770, 86. [CrossRef]

34. Strauss, M.A.; Huchra, J.P.; Davis, M.; Yahil, A.; Fisher, K.B.; Tonry, J. A redshift survey of IRAS galaxies. VII-The infrared and redshift data for the 1.936 Jansky sample. Astrophys. J. Suppl. Ser. 1992, 83, $29-63$. [CrossRef]

35. Wilman, R.J.; Edge, A.C.; Johnstone, R.M. The nature of the molecular gas system in the core of NGC 1275. Mon. Not. R. Astron. Soc. 2005, 359, 755-764. [CrossRef]

36. Scharwächter, J.; McGregor, P.J.; Dopita, M.A.; Beck, T.L. Kinematics and excitation of the molecular hydrogen accretion disc in NGC 1275. Mon. Not. R. Astron. Soc. 2013, 429, 2315-2332. [CrossRef]

37. Bernardi, M.; Alonso, M.V.; da Costa, L.N.; Willmer, C.N.A.; Wegner, G.; Pellegrini, P.S.; Rité, C.; Maia, M.A.G. Redshift-Distance Survey of Early-Type Galaxies. I. The ENEARc Cluster Sample. Astron. J. 2002, 123, 2990-3017. [CrossRef]

38. Aleksić, J.; Ansoldi, S.; Antonelli, L.A.; Antoranz, P.; Babic, A.; Bangale, P.; Barrio, J.A.; González, J.B.; Bednarek, W.; Bernardini, E.; et al. Black hole lightning due to particle acceleration at subhorizon scales. Science 2014, 346, 1080-1084. [CrossRef]

39. Smith, R.J.; Lucey, J.R.; Hudson, M.J.; Schlegel, D.J.; Davies, R.L. Streaming motions of galaxy clusters within 12,000 $\mathrm{kms}^{-1}$-I. New spectroscopic data. Mon. Not. R. Astron. Soc. 2000, 313, 469-490. [CrossRef]

40. van den Bosch, R.C.E. Unification of the fundamental plane and Super Massive Black Hole Masses. Astrophys. J. 2016, 831, 134. [CrossRef]

41. Jones, D.H.; Read, M.A.; Saunders, W.; Colless, M.; Jarrett, T.; Parker, Q.A.; Fairall, A.P.; Mauch, T.; Sadler, E.M.; Watson, F.G.; et al. The 6dF Galaxy Survey: Final redshift release (DR3) and southern large-scale structures. Mon. Not. R. Astron. Soc. 2009, 399, 683-698. [CrossRef]

42. Aharonian, F.; Akhperjanian, A.G.; Anton, G.; de Almeida, U.B.; Bazer-Bachi, A.R.; Becherini, Y.; Behera, B.; Benbow, W.; Bernlöhr, K.; Boisson, C.; et al. Discovery of Very High Energy $\gamma$-Ray Emission from Centaurus a with H.E.S.S. Astrophys. J. Lett. 2009, 695, L40-L44. [CrossRef]

43. Abdo, A.A.; Ackermann, M.; Ajello, M.; Atwood, W.B.; Baldini, L.; Ballet, J.; Barbiellini, G.; Bastieri, D.; Baughman, B.M.; Bechtol, K.; et al. Fermi Gamma-Ray Imaging of a Radio Galaxy. Science 2010, 328, 725.

44. Sahakyan, N.; Yang, R.; Aharonian, F.A.; Rieger, F.M. Evidence for a Second Component in the High-energy Core Emission from Centaurus A? Astrophys. J. Lett. 2013, 770, L6. [CrossRef]

45. Aharonian, F.; Akhperjanian, A.; Beilicke, M.; Bernlöhr, K.; Börst, H.G.; Bojahr, H.; Bolz, O.; Coarasa, T.; Contreras, J.L.; Cortina, J.; et al. Is the giant radio galaxy M 87 a TeV gamma-ray emitter? Astron. Astrophys. 2003, 403, L1-L5. [CrossRef]

46. Acciari, V.A.; Aliu, E.; Arlen, T.; Aune, T.; Beilicke, M.; Benbow, W.; Boltuch, D.; Bradbury, S.M.; Buckley, J.H.; Bugaev, V.; et al. Veritas 2008-2009 Monitoring of the Variable Gamma-ray Source M 87. Astrophys. J. 2010, 716, 819-824. [CrossRef]

47. Aleksić, J.; Alvarez, E.A.; Antonelli, L.A.; Antoranz, P.; Asensio, M.; Backes, M.; Barrio, J.A.; Bastieri, D.; Becerra González, J.; Bednarek, W.; et al. MAGIC observations of the giant radio galaxy M 87 in a low-emission state between 2005 and 2007. Astron. Astrophys. 2012, 544, A96. [CrossRef]

48. Aharonian, F.; Akhperjanian, A.G.; Bazer-Bachi, A.R.; Beilicke, M.; Benbow, W.; Berge, D.; Bernlöhr, K.; Boisson, C.; Bolz, O.; Borrel, V.; et al. Fast Variability of Tera-Electron Volt $\gamma$ Rays from the Radio Galaxy M87. Science 2006, 314, 1424-1427. [CrossRef] [PubMed] 
49. Beilicke, M.; VERITAS Collaboration. VERITAS Observations of M87 in 2011/2012. AIP Conf. Proc. 2012, 1505, 586-589.

50. Abdo, A.A.; Ackermann, M.; Ajello, M.; Asano, K.; Baldini, L.; Ballet, J.; Barbiellini, G.; Bastieri, D.; Baughman, B.M.; Bechtol, K.; et al. Fermi Discovery of Gamma-ray Emission from NGC 1275. Astrophys. J. 2009, 699, 31-39. [CrossRef]

51. Aleksić, J.; Alvarez, E.A.; Antonelli, L.A.; Antoranz, P.; Asensio, M.; Backes, M.; Barres de Almeida, U.; Barrio, J.A.; Bastieri, D.; Becerra González, J.; et al. Detection of very-high energy $\gamma$-ray emission from $<$ ASTROBJ>NGC $1275<$ /ASTROBJ> by the MAGIC telescopes. Astron. Astrophys. 2012, 539, L2.

52. Benbow, W.; VERITAS Collaboration. Highlights from the VERITAS AGN Observation Program. In Proceedings of the 34th International Cosmic Ray Conference (ICRC2015); The Hague, The Netherlands, 30 July-6 August 2015; Volume 34, p. 821.

53. Aleksić, J.; Antonelli, L.A.; Antoranz, P.; Backes, M.; Barrio, J.A.; Bastieri, D.; Becerra González, J.; Bednarek, W.; Berdyugin, A.; Berger, K.; et al. Detection of Very High Energy $\gamma$-ray Emission from the Perseus Cluster Head-Tail Galaxy IC 310 by the MAGIC Telescopes. Astrophys. J. Lett. 2010, 723, L207-L212. [CrossRef]

54. Mukherjee, R. VERITAS discovery of VHE emission from the FRI radio galaxy 3C 264. Astronomer's Telegr. 2018, 11436.

55. Dyrda, M.; Wierzcholska, A.; Hervet, O.; Moderski, R.; Janiak, M.; Ostrowski, M.; Stawarz, Ł.; for the H. E. S. S. Collaboration. Discovery of VHE gamma-rays from the radio galaxy PKS 0625-354 with H.E.S.S. ArXiv e-prints 2015, arXiv:1509.06851.

56. Fukazawa, Y.; Finke, J.; Stawarz, Ł.; Tanaka, Y.; Itoh, R.; Tokuda, S. Suzaku Observations of $\gamma$-Ray Bright Radio Galaxies: Origin of the X-Ray Emission and Broadband Modeling. Astrophys. J. 2015, 798, 74. [CrossRef]

57. Véron-Cetty, M.P.; Véron, P. A catalogue of quasars and active nuclei: 12th edition. Astron. Astrophys. 2006, 455, 773-777. [CrossRef]

58. Mueller, C.; Krauss, F.; Kadler, M.; Truestedt, J.; Ojha, R.; Ros, E.; Wilms, J.; Boeck, M.; Dutka, M.; Carpenter, B. The TANAMI program: Southern-hemisphere AGN on (sub-)parsec scales. In Proceedings of the 11th European VLBI Network Symposium AMP Users Meeting, Bordeaux, France, 9-12 October 2012; p. 20.

59. Fanaroff, B.L.; Riley, J.M. The morphology of extragalactic radio sources of high and low luminosity. Mon. Not. R. Astron. Soc. 1974, 167, 31P-36P. [CrossRef]

60. Urry, C.M.; Padovani, P. Unified Schemes for Radio-Loud Active Galactic Nuclei. Publ. Astron. Soc. Pac. 1995, 107, 803. [CrossRef]

61. Hardcastle, M.J.; Evans, D.A.; Croston, J.H. The X-ray nuclei of intermediate-redshift radio sources. Mon. Not. R. Astron. Soc. 2006, 370, 1893-1904. [CrossRef]

62. Baum, S.A.; Zirbel, E.L.; O'Dea, C.P. Toward Understanding the Fanaroff-Riley Dichotomy in Radio Source Morphology and Power. Astrophys. J. 1995, 451, 88. [CrossRef]

63. Sikora, M.; Stawarz, Ł.; Lasota, J.P. Radio Loudness of Active Galactic Nuclei: Observational Facts and Theoretical Implications. Astrophys. J. 2007, 658, 815-828. [CrossRef]

64. Fabian, A.C.; Rees, M.J. The accretion luminosity of a massive black hole in an elliptical galaxy. Mon. Not. R. Astron. Soc. 1995, 277, L55-L58.

65. Asada, K.; Nakamura, M.; Doi, A.; Nagai, H.; Inoue, M. Discovery of Sub- to Superluminal Motions in the M87 Jet: An Implication of Acceleration from Sub-relativistic to Relativistic Speeds. Astrophys. J. Lett. 2014, 781, L2. [CrossRef]

66. Kovalev, Y.Y.; Lister, M.L.; Homan, D.C.; Kellermann, K.I. The Inner Jet of the Radio Galaxy M87. Astrophys. J. Lett. 2007, 668, L27-L30. [CrossRef]

67. Hada, K.; Park, J.H.; Kino, M.; Niinuma, K.; Sohn, B.W.; Ro, H.W.; Jung, T.; Algaba, J.C.; Zhao, G.Y.; Lee, S.S.; et al. Pilot KaVA monitoring on the M 87 jet: Confirming the inner jet structure and superluminal motions at sub-pc scales. Publ. Astron. S. Jpn. 2017, 69, 71. [CrossRef]

68. Walker, R.C.; Hardee, P.E.; Davies, F.B.; Ly, C.; Junor, W. The Structure and Dynamics of the Subparsec Jet in M87 Based on 50 VLBA Observations over 17 Years at 43 GHz. Astrophys. J. 2018, 855, 128. [CrossRef]

69. Biretta, J.A.; Junor, W. The Parsec-Scale Jet in M87. Proc. Natl. Acad. Sci. USA 1995, 92, 11364-11367. [CrossRef] 
70. Meyer, E.T.; Sparks, W.B.; Biretta, J.A.; Anderson, J.; Sohn, S.T.; van der Marel, R.P.; Norman, C.; Nakamura, M. Optical Proper Motion Measurements of the M87 Jet: New Results from the Hubble Space Telescope. Astrophys. J. Lett. 2013, 774, L21. [CrossRef]

71. Giroletti, M.; Hada, K.; Giovannini, G.; Casadio, C.; Beilicke, M.; Cesarini, A.; Cheung, C.C.; Doi, A.; Krawczynski, H.; Kino, M.; et al. The kinematic of HST-1 in the jet of M 87. Astron. Astrophys. 2012, 538, L10. [CrossRef]

72. Müller, C.; Kadler, M.; Ojha, R.; Perucho, M.; Großberger, C.; Ros, E.; Wilms, J.; Blanchard, J.; Böck, M.; Carpenter, B.; et al. TANAMI monitoring of Centaurus A: The complex dynamics in the inner parsec of an extragalactic jet. Astron. Astrophys. 2014, 569, A115. [CrossRef]

73. Tingay, S.J.; Preston, R.A.; Jauncey, D.L. The Subparsec-Scale Structure and Evolution of Centaurus A. II. Continued Very Long Baseline Array Monitoring. Astron. J. 2001, 122, 1697-1706. [CrossRef]

74. Hardcastle, M.J.; Worrall, D.M.; Kraft, R.P.; Forman, W.R.; Jones, C.; Murray, S.S. Radio and X-Ray Observations of the Jet in Centaurus A. Astrophys. J. 2003, 593, 169-183. [CrossRef]

75. Marshall, H.L.; Miller, B.P.; Davis, D.S.; Perlman, E.S.; Wise, M.; Canizares, C.R.; Harris, D.E. A High-Resolution X-Ray Image of the Jet in M87. Astrophys. J. 2002, 564, 683-687. [CrossRef]

76. Lister, M.L.; Aller, M.F.; Aller, H.D.; Homan, D.C.; Kellermann, K.I.; Kovalev, Y.Y.; Pushkarev, A.B.; Richards, J.L.; Ros, E.; Savolainen, T. MOJAVE. X. Parsec-scale Jet Orientation Variations and Superluminal Motion in Active Galactic Nuclei. Astron. J. 2013, 146, 120. [CrossRef]

77. Dhawan, V.; Kellermann, K.I.; Romney, J.D. Kinematics of the Nucleus of NGC 1275 (3C 84). Astrophys. J. Lett. 1998, 498, L111-L114. [CrossRef]

78. Jorstad, S.G.; Marscher, A.P.; Morozova, D.A.; Troitsky, I.S.; Agudo, I.; Casadio, C.; Foord, A.; Gómez, J.L.; MacDonald, N.R.; Molina, S.N.; et al. Kinematics of Parsec-scale Jets of Gamma-Ray Blazars at $43 \mathrm{GHz}$ within the VLBA-BU-BLAZAR Program. Astrophys. J. 2017, 846, 98. [CrossRef]

79. Kadler, M.; Eisenacher, D.; Ros, E.; Mannheim, K.; Elsässer, D.; Bach, U. The blazar-like radio structure of the TeV source IC 310. Astron. Astrophys. 2012, 538, L1. [CrossRef]

80. Giovannini, G.; Savolainen, T.; Orienti, M.; Nakamura, M.; Nagai, H.; Kino, M.; Giroletti, M.; Hada, K.; Bruni, G.; Kovalev, Y.Y.; et al. A wide and collimated radio jet in 3C84 on the scale of a few hundred gravitational radii. Nat. Astron. 2018, 2, 472-477. [CrossRef]

81. Kim, J.Y.; Krichbaum, T.P.; Lu, R.S.; Ros, E.; Bach, U.; Bremer, M.; de Vicente, P.; Lindqvist, M.; Zensus, J.A. The limb-brightened jet of M87 down to the 7 Schwarzschild radii scale. Astron. Astrophys. 2018, 616, A188. [CrossRef]

82. Boccardi, B.; Krichbaum, T.P.; Ros, E.; Zensus, J.A. Radio observations of active galactic nuclei with mm-VLBI. Astron. Astrophys. Rev. 2017, 25, 4. [CrossRef]

83. Hada, K.; Doi, A.; Kino, M.; Nagai, H.; Hagiwara, Y.; Kawaguchi, N. An origin of the radio jet in M87 at the location of the central black hole. Nature 2011, 477, 185-187. [CrossRef] [PubMed]

84. Doeleman, S.S.; Fish, V.L.; Schenck, D.E.; Beaudoin, C.; Blundell, R.; Bower, G.C.; Broderick, A.E.; Chamberlin, R.; Freund, R.; Friberg, P.; et al. Jet-Launching Structure Resolved Near the Supermassive Black Hole in M87. Science 2012, 338, 355. [CrossRef] [PubMed]

85. Hada, K.; Kino, M.; Doi, A.; Nagai, H.; Honma, M.; Akiyama, K.; Tazaki, F.; Lico, R.; Giroletti, M.; Giovannini, G.; et al. High-sensitivity $86 \mathrm{GHz}(3.5 \mathrm{~mm})$ VLBI Observations of M87: Deep Imaging of the Jet Base at a Resolution of 10 Schwarzschild Radii. Astrophys. J. 2016, 817, 131. [CrossRef]

86. Lu, R.S.; Broderick, A.E.; Baron, F.; Monnier, J.D.; Fish, V.L.; Doeleman, S.S.; Pankratius, V. Imaging the Supermassive Black Hole Shadow and Jet Base of M87 with the Event Horizon Telescope. Astrophys. J. 2014, 788, 120. [CrossRef]

87. Rani1, B.; Jorstad, S.G.; Marscher, A.P.; Agudo, I.; Sokolovsky, K.V.; Larionov, V.M.; Smith, P.; Mosunova, D.A.; Borman, G.A.; Grishina, T.S. Exploring the connection between parsec-scale jet activity and broadband outbursts in 3C 279. Astrophys. J. 2018, 858, 80. [CrossRef]

88. Marscher, A.P.; Jorstad, S.G.; D'Arcangelo, F.D.; Smith, P.S.; Williams, G.G.; Larionov, V.M.; Oh, H.; Olmstead, A.R.; Aller, M.F.; Aller, H.D.; et al. The inner jet of an active galactic nucleus as revealed by a radio-to- $\gamma$-ray outburst. Nature 2008, 452, 966-969. [CrossRef]

89. Lott, B.; Escande, L.; Larsson, S.; Ballet, J. An adaptive-binning method for generating constant-uncertainty/constant-significance light curves with Fermi-LAT data. Astron. Astrophys. 2012, 544, A6. [CrossRef] 
90. Albert, J.; Aliu, E.; Anderhub, H.; Antoranz, P.; Armada, A.; Baixeras, C.; Barrio, J.A.; Bartko, H.; Bastieri, D.; Becker, J.K.; et al. Variable Very High Energy $\gamma$-Ray Emission from Markarian 501. Astrophys. J. 2007, 669, 862-883. [CrossRef]

91. Hada, K.; Kino, M.; Nagai, H.; Doi, A.; Hagiwara, Y.; Honma, M.; Giroletti, M.; Giovannini, G.; Kawaguchi, N. VLBI Observations of the Jet in M 87 during the Very High Energy $\gamma$-Ray Flare in 2010 April. Astrophys. J. 2012, 760, 52. [CrossRef]

92. Hada, K.; Giroletti, M.; Kino, M.; Giovannini, G.; D’Ammando, F.; Cheung, C.C.; Beilicke, M.; Nagai, H.; Doi, A.; Akiyama, K.; et al. A Strong Radio Brightening at the Jet Base of M 87 during the Elevated Very High Energy Gamma-Ray State in 2012. Astrophys. J. 2014, 788, 165. [CrossRef]

93. Marscher, A.P.; Gear, W.K. Models for high-frequency radio outbursts in extragalactic sources, with application to the early 1983 millimeter-to-infrared flare of 3C 273. Astrophys. J. 1985, 298, 114-127. [CrossRef]

94. Chidiac, C.; Rani, B.; Krichbaum, T.P.; Angelakis, E.; Fuhrmann, L.; Nestoras, I.; Zensus, J.A.; Sievers, A.; Ungerechts, H.; Itoh, R.; et al. Exploring the nature of the broadband variability in the flat spectrum radio quasar 3C 273. Astron. Astrophys. 2016, 590, A61. [CrossRef]

95. Hodgson, J.A.; Krichbaum, T.P.; Marscher, A.P.; Jorstad, S.G.; Rani, B.; Marti-Vidal, I.; Bach, U.; Sanchez, S.; Bremer, M.; Lindqvist, M.; et al. Location of $\gamma$-ray emission and magnetic field strengths in OJ 287. Astron. Astrophys. 2017, 597, A80. [CrossRef]

96. Karamanavis, V.; Fuhrmann, L.; Angelakis, E.; Nestoras, I.; Myserlis, I.; Krichbaum, T.P.; Zensus, J.A.; Ungerechts, H.; Sievers, A.; Gurwell, M.A. What can the 2008/10 broadband flare of PKS 1502+106 tell us? Nuclear opacity, magnetic fields, and the location of $\gamma$ rays. Astron. Astrophys. 2016, 590, A48. [CrossRef]

97. Aharonian, F.; Akhperjanian, A.G.; Bazer-Bachi, A.R.; Behera, B.; Beilicke, M.; Benbow, W.; Berge, D.; Bernlöhr, K.; Boisson, C.; Bolz, O.; et al. An Exceptional Very High Energy Gamma-Ray Flare of PKS 2155-304. Astrophys. J. Lett. 2007, 664, L71-L74. [CrossRef]

98. Zhang, H.; Li, X.; Guo, F.; Giannios, D. Large-amplitude Blazar Polarization Angle Swing as a Signature of Magnetic Reconnection. Astrophys. J. Lett. 2018, 862, L25. [CrossRef]

99. Petropoulou, M.; Giannios, D.; Sironi, L. Blazar flares powered by plasmoids in relativistic reconnection. Mon. Not. R. Astron. Soc. 2016, 462, 3325-3343. [CrossRef]

100. Giannios, D. Reconnection-driven plasmoids in blazars: Fast flares on a slow envelope. Mon. Not. R. Astron. Soc. 2013, 431, 355-363. [CrossRef]

101. Petropoulou, M.; Sironi, L. The Steady Growth of the High-Energy Spectral Cutoff in Relativistic Magnetic Reconnection. Mon. Not. Roy. Astron. Soc. 2018, 481, 4. [CrossRef]

102. Kagan, D.; Sironi, L.; Cerutti, B.; Giannios, D. Relativistic Magnetic Reconnection in Pair Plasmas and Its Astrophysical Applications. Space Sci. Rev. 2015, 191, 545-573. [CrossRef]

103. Hirotani, K.; Pu, H.Y. Energetic Gamma Radiation from Rapidly Rotating Black Holes. Astrophys. J. 2016, 818, 50. [CrossRef]

104. Rieger, F.M. Nonthermal Processes in Black Hole-Jet Magnetospheres. Int. J. Modern Phys. D 2011, 20, 1547-1596. [CrossRef]

105. Blandford, R.D.; Znajek, R.L. Electromagnetic extraction of energy from Kerr black holes. Mon. Not. R. Astron. Soc. 1977, 179, 433-456. [CrossRef]

106. Katsoulakos, G.; Rieger, F.M. Magnetospheric Gamma-Ray Emission in Active Galactic Nuclei. Astrophys. J. 2018, 852, 112. [CrossRef]

107. Blandford, R.; Yuan, Y.; Hoshino, M.; Sironi, L. Magnetoluminescence. Space Sci. Rev. 2017, 207, $291-317$. [CrossRef]

108. Blandford, R.; East, W.; Nalewajko, K.; Yuan, Y.; Zrake, J. Active Galactic Nuclei: The TeV Challenge. arXiv 2015, arXiv:1511.07515.

109. McKinney, J.C.; Tchekhovskoy, A.; Blandford, R.D. Alignment of Magnetized Accretion Disks and Relativistic Jets with Spinning Black Holes. Science 2013, 339, 49. [CrossRef] [PubMed]

110. Zamaninasab, M.; Clausen-Brown, E.; Savolainen, T.; Tchekhovskoy, A. Dynamically important magnetic fields near accreting supermassive black holes. Nature 2014, 510, 126-128. [CrossRef] [PubMed]

111. Liska, M.; Hesp, C.; Tchekhovskoy, A.; Ingram, A.; van der Klis, M.; Markoff, S. Formation of precessing jets by tilted black hole discs in 3D general relativistic MHD simulations. Mon. Not. R. Astron. Soc. 2018, 474, L81-L85. [CrossRef] 
112. Zhang, H.; Li, H.; Guo, F.; Taylor, G. Polarization Signatures of Kink Instabilities in the Blazar Emission Region from Relativistic Magnetohydrodynamic Simulations. Astrophys. J. 2017, 835, 125. [CrossRef]

113. Marscher, A.P. Turbulent, Extreme Multi-zone Model for Simulating Flux and Polarization Variability in Blazars. Astrophys. J. 2014, 780, 87. [CrossRef]

114. Acharya, B.S.; Agudo, I.; Al Samarai, I.; Alfaro, R.; Alfaro, J.; Alispach, C.; Alves Batista, R.; Amans, J.P.; Amato, E.; Ambrosi, G.; et al. Science with the Cherenkov Telescope Array. arXiv 2017, arXiv:1709.07997.

115. IceCube Collaboration; Aartsen, M.G.; Ackermann, M.; Adams, J.; Aguilar, J.A.; Ahlers, M.; Ahrens, M.; Al Samarai, I.; Altmann, D.; Andeen, K.; et al. Multimessenger observations of a flaring blazar coincident with high-energy neutrino IceCube-170922A. Science 2018, 361, eaat1378.

(C) 2019 by the authors. Licensee MDPI, Basel, Switzerland. This article is an open access article distributed under the terms and conditions of the Creative Commons Attribution (CC BY) license (http:/ / creativecommons.org/licenses/by/4.0/). 


\title{
Centaurus A: Hard X-ray and High-Energy Gamma-Ray Light Curve Correlation
}

\author{
Isak Delberth Davids 1,2, ${ }^{\text {, Markus Böttcher }}{ }^{2}$ and Michael Backes ${ }^{1,2}$ \\ 1 Department of Physics, University of Namibia, Private Bag 13301, Windhoek 12010, Namibia; \\ mbackes@unam.na \\ 2 Centre for Space Research, North-West University, Private Bag X6001, Potchefstroom 2520, South Africa; \\ Markus.Bottcher@nwu.ac.za \\ * Correspondence: isak.davids@gmail.com
}

Received: 26 February 2019; Accepted: 27 March 2019; Published: 4 April 2019

\begin{abstract}
Centaurus A, powered by a 55 million solar mass supermassive black hole, has been intensively monitored in all accessible wavelength ranges of the electromagnetic spectrum. However, its very-high energy gamma $(\gamma)$ ray flux (TeV photons), obtained from H.E.S.S. is relatively faint, hampering detailed light curve analyses in the most energetic energy band. Yet, the extensive long-term light curve data from Fermi-LAT and Swift-BAT (hard X-rays) allows for cross-correlation studies. We find a hint that $X$-ray emission from Centaurus A precedes the $\gamma$ rays by $25 \pm 125$ days. If this lag is real and related to a $\gamma \gamma$ absorption effect in the broad-line region (BLR) around the central source, we can constrain the size of the BLR using light-travel time arguments. These are first results of extended light curve correlation studies between high-energy $\gamma$ rays and $\mathrm{X}$-rays from Centaurus A.
\end{abstract}

Keywords: active galactic nucleus; radio galaxy; Centaurus $\mathrm{A} ; \mathrm{GeV} \gamma$-rays; $\mathrm{TeV} \gamma$-rays; light curve; discrete correlation function

\section{Introduction}

Active galactic nuclei (AGN) are a type of galaxies hosting extremely luminous central regions that can outshine the entire galaxy. It is customarily accepted that these active central regions are powered by a super-massive black hole (SMBH). The SMBH accretes matter from its surroundings through an accretion disk around it. AGN are known to feature a relativistic outflow, in the form of jets, of charged particles traveling in a highly collimated flow that slams into the intergalactic matter forming large radio lobes at the ends.

AGN can be classified based on the observer's viewing angle [1] with respect to the AGN jet orientation. Radio galaxies (RGs) belong to the type of AGN for which the jet viewing angle with respect to our line of sight is relatively large in contrast to blazars for which this angle is small. A well known radio galaxy is Centaurus A (Cen A), which is the source of interest in this study.

In general, radio-loud AGN feature a broadband (from radio to $\gamma$ rays) emission spectrum which is primarily attributed to non-thermal radiation. Two distinct peaks can be seen in such a spectral energy distribution (SED) where the lower energy peak (extending from radio to X-rays) is traditionally accepted as being due to synchrotron emission from relativistic electrons in the jets. In leptonic models, the second hump (from X-rays to $\gamma$ rays) in the SED is attributed to inverse-Compton upscattering of a soft target photon field by the same relativistic jet electron population.

Amongst the basic components of the structure of a typical AGN, are the fast-moving high-density clouds surrounding the central engine (but external to the jet) and located closer to the central SMBH than the optically obscuring dust torus. Due to the broad emission lines from this region, observed primarily in the optical and ultraviolet, these components are referred to as the broad line regions (BLRs) of the AGN (see [2] for a discussion on the possible origins of the BLR). The location of the 
BLR can be measured by the reverberation method employing the light travel time, the width of the emission line which in turn indicates the speed of the BLR clouds, and the observable temporal lag between the ionizing continuum radiation from the accretion disk and the line emission. The intense BLR radiation field is likely to be an efficient target photon field for $\gamma \gamma$-absorption of high-energy (HE; $E \sim 0.1-100 \mathrm{GeV}$ ) and / or very-high energy (VHE; $E \gtrsim 100 \mathrm{GeV}$ ) $\gamma$-rays producing electron-positron $\left(e^{-} e^{+}\right)$pairs $[3,4]$.

In this work, we report results of the time-series study using the discrete cross-correlation of light curves as applied to Swift-BAT X-ray and Fermi-LAT $\gamma$-ray long-term observations of the nearest radio galaxy, Cen A.

\section{Centaurus A}

The massive elliptical galaxy NGC 5128 (discovered in 1826, and also known as Cen A), is the closest radio galaxy, at a distance of $3.4 \mathrm{Mpc}$ [5]. This source is centrally powered by a SMBH of mass $5.5 \times 10^{7} \mathrm{M}_{\odot}$ (see e.g., [6,7]). The giant radio lobes of Cen A subtend $\sim 10^{\circ}$ on the sky due to its proximity to Earth. This Fanaroff-Riley Type I [8] radio galaxy has been observed from radio to VHE $\gamma$ rays, and is an ideal target for studying radio lobes and relativistic outflows.

Long-term multi-wavelength observations of Cen A from various astronomical facilities lead to high-quality long-term light curves in several wavebands across the electromagnetic spectrum.

\section{The Spectral Energy Distribution of Cen A}

Numerous extensive studies have so far been carried out on Cen A, aiming at characterizing the SED of the source (see e.g., [5,9-12]). As is seen with other active galaxies, the SED of Cen A is dominated by non-thermal radiative output originating mainly from synchrotron and inverse-Compton emission mechanisms.

In [9], the broadband SED of Cen A is reported, ranging from radio to VHE $\gamma$-rays modeled with various theoretical emission processes. A break in the $\gamma$-ray spectrum of the source indicating a spectral upturn above $2.4 \mathrm{GeV}$ has been established [10]. This break is not well modeled using a single leptonic synchrotron self-Compton model. Recent results, from joint observations by H.E.S.S. and Fermi-LAT [11], show that the VHE data points are consistently above the HE power-law extrapolation of the $\gamma$-ray spectrum measured below the break. There is clear evidence for a second spectral component in VHE $\gamma$ rays in the SED.

In the so-called one-zone model, one considers a single emission region (say a relativistic blob traveling along the jet) producing $\gamma$ rays by electron-induced upscattering of low energy photons through the inverse-Compton process. The target low energy photon populations can be internal or external to the jet system. The single-zone models generally fail to reproduce the VHE $\gamma$ ray data of Cen A.

Also shown in [9,12], one can invoke a second emission zone with differing input parameters to account for the VHE data points. Such a two-zone leptonic scenario has been recently employed by the Fermi-LAT and H.E.S.S. collaborations to fully explain the entire $\gamma$-ray spectrum of Cen A [11].

Alternatively, the hadronic explanation of the HE peak uses energetic protons (interacting with low-energy photons) that produce $\gamma$-rays after photo-pion production that leads to pion and muon decay processes (or from proton-synchrotron radiation). These hadronic models will typically require an input of rather large power in the proton population (see e.g., [13]), reaching $10^{47}-10^{49} \mathrm{erg} \cdot \mathrm{s}^{-1}$. The corresponding luminosity estimates are much lower, by even two or more orders of magnitude for leptonic models—-this is essentially attributed to electrons radiating more efficiently.

A combination of leptonic and hadronic (leptohadronic) models has also been used in a successful reproduction of the $\mathrm{HE}$ tail of the SED of Cen $\mathrm{A}$ where the TeV component is considered as the emission from a relativistic proton population that interacts with the photons (photohadronic interaction) produced by the primary leptonic component [12]. 


\section{$\gamma \gamma$ Absorption}

The $\mathrm{TeV}$ photons that result from inverse-Compton scattering can again undergo interactions with soft photons from various photon fields producing electron-positron pairs. The target photon field can be external to the jet-in particular an intense photon population is provided by the BLR of an AGN. Other external regions with soft photons include the accretion disk, the dusty torus, the cosmic microwave background, and the extragalactic background light.

Due to the potentially large density of the BLR radiation field, it is possible that $\gamma \gamma$ absorption by the BLR radiation field produces a discernible spectral signature. Indeed, $\gamma \gamma$ absorption in the BLR of blazars has been considered by several authors, see e.g., $[3,4,14]$, requiring that the $\gamma$-ray emission region must be located outside or at the outer edge of the BLR, as significant $\gamma \gamma$ absorption by the BLR can be avoided by having the $\gamma$-ray emission region outside the BLR.

\section{Multi-wavelength Observations}

To study the long-term activity of Cen A, we obtained contemporaneous data spanning about 6.8 years of hard $\mathrm{X}$-rays and high-energy $\gamma$ rays.

\subsection{X-ray Data}

Hard X-ray data (15-150 keV) from the Burst Alert Telescope (BAT) [15] on board the Neil Gehrels Swift Observatory was used to obtain a 6.8-year span light curve. While primarily designed for gamma-ray burst (GRB) detection and rapid triggering, BAT is an efficient hard X-ray monitor instrument $[16,17]$. These data are readily available from the BAT transient source online repository ${ }^{1}$, and have been used herein to produce the light curve binned in 14 day bins in the period from MJD 54703.6 to MJD 57195.6 (i.e., between 8 August 2008 and 22 June 2015). The starting date of this time window is set by the mission operation date of the Fermi-LAT, and the ending date by the time of analyses in the year 2015.

\subsection{HE Gamma-ray Data}

High-energy $\gamma$-ray $(100 \mathrm{MeV}-500 \mathrm{GeV})$ observations of Cen A that were contemporaneous to the Swift-BAT data, from the Large Area Telescope (LAT), the primary instrument on board the Fermi gamma-ray Space Telescope (Fermi) [18], were used to extract the $\gamma$-ray light curve. We employed the publicly available event reconstruction tool (Fermi-LAT Pass 8 event-level analysis) [19], which has been optimized for point-like sources after a comprehensive review of $\gamma$-ray event analysis. Released in 2015, Pass 8 is considered to be the event reconstruction and analysis realizing the full scientific potential of the instrument by increasing the effective area, improving the point spread function, and widening the energy range to which LAT is sensitive. We performed unbinned likelihood analysis (a maximum likelihood optimization technique) as described in the LAT Cicerone (see [20]) to obtain the light curve in the energy range 0.1 to $500 \mathrm{GeV}$ within a $10^{\circ}$ region of interest centered at the position of Cen A. The LAT science tools include all Pass 8 background models, such as the galactic diffuse emission and the extragalactic isotropic diffuse emission models.

Figure 1 shows the $\mathrm{X}$-ray and $\gamma$-ray light curves produced as described above. The light curves span 6.8 years of observations, sampled in 14 day bins.

1 The publicly available monitor web page, http://swift.gsfc.nasa.gov/docs/swift/results/transients/, provides light curves for astrophysical sources observed with Swift-BAT. 


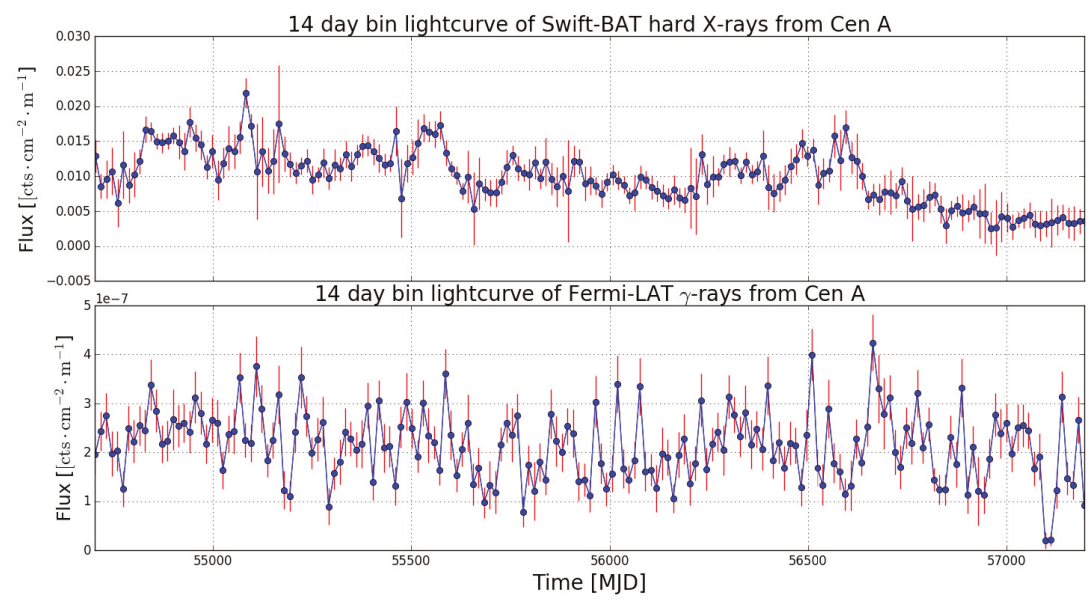

Figure 1. The Swift-burst alert telescope (BAT) and Fermi-large area telescope (LAT) light curves spanning 6.8 years between 2008 and 2015 (at a 14 day sample rate) is shown here.

\section{Time-lag Analysis}

\subsection{The Discrete Correlation Function}

We now test for the presence of a correlation (or absence thereof) between the two observed time varying signals from Cen A. For this purpose, we employ the discrete correlation function (DCF) analysis developed by [21]. One of the advantages of the DCF is that it does not interpolate data and can also handle sparse and / or unevenly sampled light curves.

Suppose we have recorded two time series of time-dependent data $a(t)$ and $b(t)$ (these can be in the form of astronomical fluxes), then the mathematical description of the set of unbinned discrete correlations is

$$
\mathrm{UDCF}_{i j}=\frac{\left(a_{i}-\bar{a}\right)\left(b_{j}-\bar{b}\right)}{\left[\left(\sigma_{a}^{2}-e_{a}^{2}\right)\left(\sigma_{a}^{2}-e_{b}^{2}\right)\right]^{1 / 2}},
$$

where $a_{i}$ and $b_{j}$ are observed flux pairs, such that the corresponding pairwise lag is $\Delta t_{i j}=t_{j}-t_{i}$. The averages of the series are denoted with $\bar{a}$ and $\bar{b}$, while $\sigma_{a}^{2}$ and $\sigma_{b}^{2}$ represent their variances. The errors in these measurements are denoted by $e_{a}$ and $e_{b}$. DCF values were computed for pairwise time-lags ( $M$ pairs in total) where each time-lag is given by $\Delta t_{i j}=t_{j}-t_{i}$. The DCF that we make use of here was then obtained through binning $U D C F_{i j}$ in time and averaging over all $M$ pairs of $U D C F_{i j}$ for which $\tau-\Delta \tau / 2 \leq\left|\Delta t_{i j}\right| \leq \tau+\Delta \tau / 2$ to get

$$
D C F(\tau)=\frac{U D C F_{i j}}{M} .
$$

As can be seen in [21], the uncertainty in the DCF is obtained by

$$
\sigma_{\mathrm{DCF}}(\tau)=\frac{1}{M-1} \sqrt{\sum\left(\mathrm{UDCF}_{i j}-\mathrm{DCF}(\tau)\right)^{2}}
$$

where the summation is over all series of unbinned discrete correlations obtained in Equation (1).

The algorithm employed progressively iterates through time-lags and computes a discrete correlation coefficient for each lag. This enables an evaluation of the correlation between the two 
time series as a function of difference in arrival time of the signals. The result can be interpreted as trailing or leading of the first input signal if the lag is positive or negative, respectively. The concept of auto-correlation is achieved if $a(t)=b(t)$.

\subsection{Monte Carlo Simulation of Light Curves}

In order to quantify the statistical significance of the DCF computed, we used the observed light curve in each band to simulate, via a Monte Carlo approach, a set of 20,000 artificial light curves. To accomplish this, an algorithm proposed by [22] and later improved by [23], was used. A Python implementation of this approach is published by [24].

For each observed light curve (the HE $\gamma$-ray and X-ray curves), we used the algorithm by [24] to extract the underlying power spectral densities (PSDs) and probability density functions (PDFs). These PSDs and PDFs are then used to simulate light curves that resemble the observations. At first, to get a PSD, the algorithm derives the associated periodogram (the power as function of frequency) which is in-turn fitted with a normalized (by $A$ ) smoothly bending power-law (around $v_{\text {low }}$ and $v_{\text {high }}$ ) to which a constant $c$ is added. Secondly, the PDF is obtained again from a best-fit (to its histogram) of a superposition of two distributions (a gamma-distribution $\Gamma(\kappa, \theta)$ and $\log$-normal distribution $\ln N\left(\mu, \sigma^{2}\right)$ ) whose contributions are weighted by $\omega_{\Gamma}$. The latter weighing takes care of the $\omega_{\ln N}=1-\omega_{\Gamma}$ partitioning of the mixing distributions towards the PDF. Table 1 shows the description and the numerical values of the above-mentioned parameters as used in this work.

Table 1. An illustration of the parameter set for simulating the $\gamma$-ray light curves in the Python implementation [24] of the algorithm by [23]. In this case, amongst others one, notes the over $80 \%$ contribution of the $\Gamma$-distribution to the probability density function (PDF) at the expense of the log-normal component.

\begin{tabular}{lcc}
\hline Parameter & Symbol & Numerical Value \\
\hline PSD normalizer & $A$ & $0.03 \mathrm{~Hz}^{-1}$ \\
Bending frequency of PSD & $v_{\mathrm{b}}$ & $2.3 \times 10^{-4} \mathrm{~Hz}$ \\
Low frequency slope in PSD & $v_{\text {low }}$ & 1.1 \\
High frequency slope in PSD & $v_{\text {high }}$ & 2.2 \\
Constant added for PSD & $c$ & 0.009 \\
Shape parameter of $\Gamma$-distribution for PDF & $\kappa$ & 5.67 \\
Scale parameter of $\Gamma$-distribution for PDF & $\theta$ & 5.96 \\
Mean of the log-normal distribution for PDF & $v$ & 2.14 \\
Variance of the log-normal distribution for PDF & $\sigma^{2}$ & 0.31 \\
Weight between the $\Gamma$ and log-normal distributions for PDF & $\omega_{\Gamma}$ & 0.82 \\
\hline
\end{tabular}

In this way, we simulated two pairs of 20,000 light curves resembling the $\gamma$-ray and the X-rays, respectively. We then obtained correlation coefficients (using the [21] framework) by correlating each of the 20,000 simulated light curves against the observed light curve in the other band. The simulated light curves feature the same variability and statistical properties as the observed light curves. Using the Monte Carlo simulated light curves, we sorted the lag-wise correlation coefficients and determined the upper $5 \%$ of these, which is used then to define the upper boundary of the $95 \%$ confidence level (CL). These limits derived from the contour boundaries of the CLs of the DCF process are used as a statistical significance check of the peak of the DCF distribution obtained.

\subsection{DCF Computation Result}

Figure 2 shows the discrete correlation function between hard X-rays and $\mathrm{HE} \gamma$ rays from Cen A. This is the correlation coefficient as a function of time-lag where the correlation was performed as described in Section 4.1. The shape of the peak in Figure 2 is approximately Gaussian for which the width is a measure of the uncertainty in the time lag. Overlaid on the correlation, is a band delimiting the $95 \%$ confidence level (see Section 4.2 ) which is significantly surpassed by the peak of the 
correlation function. It can be noticed that the result hints to a scenario where the X-rays observed from the Swift-BAT instrument lead the Fermi-LAT $\gamma$-rays by $25 \pm 125$ days (see the peak of the Gaussian fit which has a standard deviation of 125 days). The correlation coefficient at the peak is $0.35 \pm 0.14$. The result is established to have been significant above the $95 \% \mathrm{CL}$, but a correlation at a $99.7 \mathrm{CL}$ significance cannot be claimed due to the magnitude of the error bars.

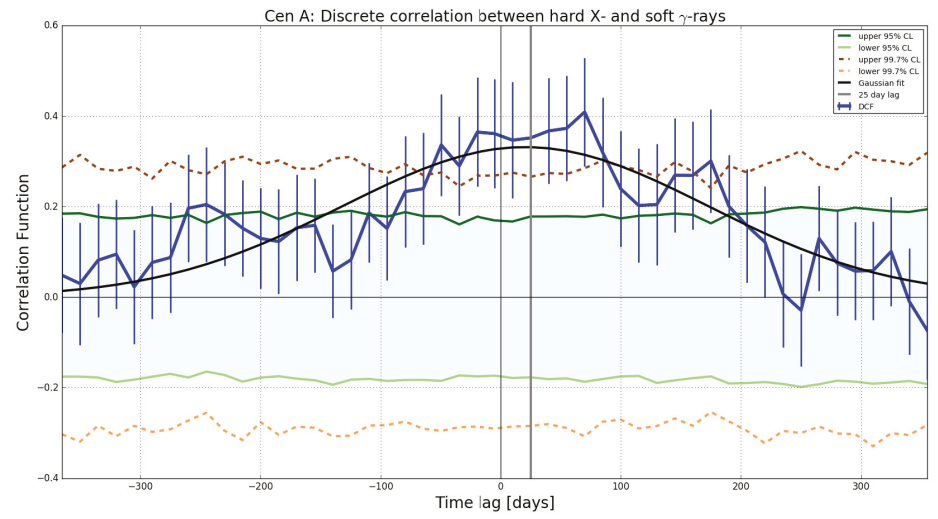

Figure 2. Discrete correlation function (DCF) result: the solid blue curve denotes the DCF as a function of time-lag (fitted with a Gaussian function in black), while the error bars on the correlation coefficients are propagated from the errors on the flux measurements. The peak at $25 \pm 125$ day time lag (indicated by the solid gray vertical line), is the deduced time-lag by which $\mathrm{HE} \gamma$-rays trail behind the X-rays. The solid dark green line traces out the upper 95\% confidence level (CL), while light green solid line marks the lower boundary of the $95 \%$ CL. Also shown are the upper and lower $99.7 \%$ CLs in dark brown and light brown, respectively. The lag bin width of the shown DCF is 15 days, constrained by the observation sampling rate and the duration of the observations. The latter also serves as a boundary to the lag range tested. This presented choice of the lag bin width and its range were arrived at by iterative visual inspection through these intervals. The correlation is insignificant on time scales larger than the time scale in this window.

\section{Summary and Discussion}

Cen A, the closest radio galaxy to Earth, has been observed in various wavelength bands across the electromagnetic spectrum over the years.

The light curve correlation between the 6.8 years of HE gamma-ray and the X-ray data of Cen A considered here, resulted in a hint of a possible time-lag of about $25 \pm 125$ days of the gamma-ray emission behind the X-rays. The discrete correlation function (DCF) associated with this lag peaked at the value of $0.35 \pm 0.14$. Although the time-lag is a mere hint (due to the very broad Gaussian width of the peak), the correlation is above the $95 \% \mathrm{CL}$ as obtained from the correlation studies done on the Monte Carlo simulated light curves.

In the event that further studies confirm a time-lag of this magnitude, then we can couple that to arguments of light-travel time, to set a constraint on the size of the BLR of the system. This is based on the argument that, while the emission region is within the BLR, X-rays produced by the relativistic particles escape immediately while $\gamma$ rays are $\gamma \gamma$ absorbed. The resulting lag $\left(t_{\text {lag }}\right)$ is then associated to the size of the BLR $\left(R_{\mathrm{BLR}}\right)$ through

$$
\begin{aligned}
\Delta R_{\mathrm{BLR}} & \sim c \Delta t_{\mathrm{lag}} \\
& \sim(6.5 \pm 32.4) \times 10^{16} \mathrm{~cm} .
\end{aligned}
$$


This (25 light-day distance) would then indicate that the size of the BLR near the central engine is about 0.02 parsec. In this estimate, we neglect relativistic beaming for a typical radio galaxy for which the jet is largely misaligned to our line of sight.

Estimates of $R_{\mathrm{BLR}}$ of AGN have been set through a relationship with the observed luminosity of the accretion disk (see e.g., [25]) here written in the form of

$$
R_{\mathrm{BLR}} \sim 3 \times 10^{17}\left(L_{d_{45}}\right)^{1 / 2} \mathrm{~cm},
$$

where $L_{d_{45}}$ is the disk luminosity in units of $10^{45} \mathrm{erg} / \mathrm{s}$. This relationship implies that AGN with luminosities in the range $10^{43} \mathrm{erg} / \mathrm{s} \lesssim L_{d} \lesssim 10^{45} \mathrm{erg} / \mathrm{s}$ feature BLR of sizes in the range of 12 to 116 light-days. This is consistent with the hint from the current work of the BLR in Cen A to be about 25 light-days.

Author Contributions: The formal analysis and the orgininal draft preparation was carried out by I.D.D., while M.B. (Michael Backes) and M.B. (Markus Böttcher) contributed with validation, conceptualization, reviewing, editing and supervision. Furthermore, M.B. (Markus Böttcher) was pivotal in funding acquisition for the project.

Funding: This project is sponsored by the German Academic Exchange Service (DAAD) (German: Deutscher Akademischer Austauschdienst), the National Research Foundation (NRF) and the Department of Science and Technology of the Republic of South Africa through the South African Research Chair Initiative under SARChI grant 64798, as well as the Department of Physics of the University of Namibia.

Conflicts of Interest: The authors declare no conflict of interest.

\section{Abbreviations}

The following abbreviations are used in this manuscript:

BAT Burst Alert Telescope (on-board Neil Gehrels Swift Observatory)

BLR Broad line region

CL Confidence level

DCF Discrete correlation function

HE High-energy (gamma-rays of energy 0.1-100 GeV)

H.E.S.S. High-Energy Stereoscopic System

IACT Imaging Atmospheric Cherenkov Telescope

LAT Large Area Telescope (on-board Fermi satellite)

RG Radio galaxy

SED Spectral energy distribution

VHE Very high-energy (gamma-rays of energy $\gtrsim 100 \mathrm{GeV}$ )

\section{References}

1. Urry, C.M.; Padovani, P. Unified Schemes for Radio-Loud Active Galactic Nuclei. Publ. Astron. Soc. Pac. 1995, 107, 803. [CrossRef]

2. Czerny, B.; Hryniewicz, K. The origin of the broad line region in active galactic nuclei. Astron. Astrophys. 2011, 525, L8. [CrossRef]

3. Roustazadeh, P.; Boettcher, M. Compton and Synchrotron Emission from Pair Cascades in Radio Galaxies. AAS/High Energy Astrophys. Div. 2011, 12, 4.08

4. Böttcher, M.; Els, P. Gamma-Gamma Absorption in the Broad Line Region Radiation Fields of Gamma-ray Blazars. Astrophys. J. 2016, 821, 102-106. [CrossRef]

5. Israel, F.P. Centaurus A - NGC 5128. Astron. Astrophys. Rev. 1998, 8, 237-278. [CrossRef]

2 Disclaimer: Any opinion, finding and conclusion or recommendation expressed in this material is that of the authors and the NRF does not accept any liability in this regard. 
6. Marconi, A.; Pastorini, G.; Pacini, F.; Axon, D. J.; Capetti, A.; Macchetto, D.; Koekemoer, A.M.; Schreier, E.J. The supermassive black hole in Centaurus A: A benchmark for gas kinematical measurements. Astron. Astrophys. 2006, 448, 921-953. [CrossRef]

7. Häring-Neumayer, N.; Cappellari, M.; Rix, H.-W.; Hartung, M.; Prieto, M.A.; Meisenheimer, K.; Lenzen, R. VLT Diffraction-limited Imaging and Spectroscopy in the NIR: Weighing the Black Hole in Centaurus A with NACO. Astrophys. J. 2006, 643, 226-237. [CrossRef]

8. Fanaroff, B.L.; Riley, J.M. The morphology of extragalactic radio sources of high and low luminosity. Mon. Not. R. Astron. Soc. 1974, 167, 31P-36P. [CrossRef]

9. Abdo, A.A.; Ackermann, M.; Ajello, M.; Atwood, W.B.; Baldini, L.; Ballet, J.; Barbiellini, G.; Bastieri, D.; Baughman, B.M.; Bechto, K.; et al. Fermi Large Area Telescope View of the Core of the Radio Galaxy Centaurus A. Astrophys. J. 2010, 719, 1433-1444. [CrossRef]

10. Graham, J.A.; Brown, A.M.; Boehm, C.; Lacroix, T.; Chadwick, P.M.; Joseph, S. The Fermi-LAT spectrum of Centaurus A-Analysis and interpretations. AIP Conf. Proc. 2017, 1792, 050004.

11. Abdalla, H.; Abramowski, A.; Aharonian, F.; Benkhali, F.A.; Angüner, E.O.; Arakawa, M.; Armand, C.; Arrieta, M.; Backes, M.; Balzer, A.; et al. The $\gamma$-ray spectrum of the core of Centaurus A as observed with H.E.S.S. and Fermi-LAT. Astron. Astrophys. 2018, 619, A71.

12. Petropoulou, M.; Lefa, E.; Dimitrakoudis, S.; Mastichiadis, A. One-zone synchrotron self-Compton model for the core emission of Centaurus A revisited. Astron. Astrophys. 2014, 562, A12. [CrossRef]

13. Böttcher, M.; Reimer, A.; Sweeney, K.; Prakash, A. Leptonic and Hadronic Modeling of Fermi-detected Blazars. Astrophys. J. 2013, 768, 54. [CrossRef]

14. Protheroe, R.J.; Biermann, P.L. Photon-photon absorption above a molecular cloud torus in blazars. Astropart. Phys. 1997, 6, 293-300. [CrossRef]

15. Gehrels, N.; Chincarini, G.; Giommi, P.; Mason, K.O.; Nousek, J.A.; Wells, A.A.; White, N.E.; Barthelmy, S.D.; Burrows, D.N.; Cominsky, L.R.; et al. The Swift Gamma-Ray Burst Mission. Astrophys. J. 2004, 611, 1005-1020. [CrossRef]

16. Krimm, H.A.; Barthelmy, S.D.; Markwardt, C.B.; Sanwal, D.; Tueller, J.; Gehrels, N.; Swift/BAT Team. The Swift-BAT Hard X-ray Transient Monitor. Bull. Am. Astron. Soc. 2006, 38, 374.

17. Krimm, H.A.; Holland, S.T.; Corbet, R.H.D.; Pearlman, A.B.; Romano, P.; Kennea, J.A.; Bloom, J.S.; Barthelmy, S.D.; Baumgartner, W.H.; Cummings, J.R.; et al. The Swift/BAT Hard X-Ray Transient Monitor. Astrophys. J. Suppl. Ser. 2013, 209, 14. [CrossRef]

18. Abdo, A.A.; Ackermann, M.; Althouse, W.; Anderson, B.; Axelsson, M.; Baldini, L.; Ballet, J.; Band, D.L.; Barbiellini, G.; et al. The Large Area Telescope on the Fermi Gamma-Ray Space Telescope Mission. Astrophys. J. 2009, 697, 1071-1102.

19. Atwood, W.; Albert, A.; Baldini, L.; Tinivella, M.; Bregeon, J.; Pesce-Rollins, M.; Sgrò, C.; Bruel, P.; Charles, E.; Drlica-Wagner, A.; et al. Pass 8: Toward the Full Realization of the Fermi-LAT Scientific Potential. arXiv 2013, arXiv:1303.3514.

20. Abdo, A.A.; Ackermann, M.; Ajello, M.; Atwood, W.B.; Axelsson, M.; Baldini, L.; Ballet, J.; Band, D.L.; Barbiellini, G.; Bastieri, D.; et al. Fermi/Large Area Telescope Bright Gamma-Ray Source List. Astrophys. J. Suppl. Ser. 2009, 183, 46-66. [CrossRef]

21. Edelson, R.A.; Krolik, J.H. The discrete correlation function: A new method for analyzing unevenly sampled variability data. ESA Spec. Public. 1988, 281, 646-659. [CrossRef]

22. Timmer, J.; Koenig, M. On generating power law noise. Astron. Astrophys. 1995, 300, 707.

23. Emmanoulopoulos, D.; McHardy, I.M.; Papadakis, I.E. Generating artificial light curves: Revisited and updated. Mon. Not. R. Astron. Soc. 2013, 433, 907-927. [CrossRef]

24. Connolly, S. A Python Code for the Emmanoulopoulos et al. [arXiv:1305.0304] Light Curve Simulation Algorithm. arXiv 2015, arXiv:1503.06676.

25. Bentz, M.C.; Peterson, B.M.; Netzer, H.; Pogge, R.W; Vestergaard, M. The Radius-Luminosity Relationship for Active Galactic Nuclei: The Effect of Host-Galaxy Starlight on Luminosity Measurements. II. The Full Sample of Reverberation-Mapped AGNs. Astrophys. J. 2009, 697, 160. [CrossRef]

(C) 2019 by the authors. Licensee MDPI, Basel, Switzerland. This article is an open access article distributed under the terms and conditions of the Creative Commons Attribution (CC BY) license (http:/ / creativecommons.org/licenses/by/4.0/). 


\title{
3C 84: Observational Evidence for Precession and a Possible Relation to TeV Emission
}

\author{
Silke Britzen ${ }^{1, *}$, Christian Fendt ${ }^{2}$, Michal Zajaček ${ }^{1,3,4}$, Frédéric Jaron ${ }^{1,5}$, Ilya Pashchenko ${ }^{6}$, \\ Margo F. Aller ${ }^{7}$ and Hugh D. Aller ${ }^{7}$ \\ 1 Max-Planck-Institut für Radioastronomie, Auf dem Hügel 69, 53121 Bonn, Germany \\ 2 Max Planck Institute for Astronomy, Königstuhl 17, 69117 Heidelberg, Germany \\ 3 I. Physikalisches Institut, Universität Köln, Zülpicher Str. 77, 50937 Köln, Germany \\ 4 Center for Theoretical Physics, Polish Academy of Sciences, Al. Lotników 32/46, 02-668 Warsaw, Poland \\ 5 Institute of Geodesy and Geoinformation, University of Bonn, Nußallee 17, 53115 Bonn, Germany \\ 6 Astro Space Center, Lebedev Physical Institute, Russian Academy of Sciences, 84/32 Profsoyuznaya st., \\ 117997 Moscow, Russia \\ 7 Astronomy Department, University of Michigan, Ann Arbor, MI 48109-1107, USA \\ * Correspondence: sbritzen@mpifr.de
}

Received: 13 February 2019; Accepted: 28 July 2019; Published: 14 August 2019

\begin{abstract}
C 84 (NGC 1275, Perseus A) is a bright radio source at the center of an ongoing merger, where HST observations show two colliding spiral galaxies. 3C 84 holds promise to improve our understanding about how of the activity of active galactic nuclei, the formation of supermassive binary black holes, feedback processes, and galaxy collisions are interrelated. 3C,84 is one of only six radio galaxies, which reveal $\mathrm{TeV}$ emission. The origin of this $\mathrm{TeV}$ emission is still a matter of debate. Our present study is based on high resolution radio interferometric observations (15 GHz) of the pc-scale jet in this complex radio galaxy. We have re-modeled and re-analyzed 42 VLBA observations of 3C 84, performed between 1999.99 and 2017.65. In order to enable a proper alignment of the VLBA observations, we developed a method of a "differential" alignment whereby we select one reference point and minimize the deviations from this reference point in the remaining epochs. As a result, we find strong indication for a precession of the $3 C 84$ jet-not only for its central regions, but also for the outer lobe at 10 mas distance. These findings are further supported by our kinematic precession modeling of the radio flux-density monitoring data provided by the University of Michigan Radio Observatory and the Owens Valley Radio Observatory, which yields a precession time scale of about $40 \mathrm{yr}$. This time scale is further supported by literature maps obtained about $40 \mathrm{yr}$ ago (1973 and 1974.1) which reveal a similar central radio structure. We suggest that the TeV flare detected by MAGIC may correlate with the precession of $3 \mathrm{C} 84$, as we disentangle a projected reversal point of the precessing motion that correlates with the flaring time. This may physically be explained by a precessing jet sweeping over a new region of so far undisturbed X-ray gas which would then lead to shock-produced TeV-emission. In addition, we perform a correlation analysis between the radio data and $\mathrm{GeV}$ data obtained by the Fermi Gamma-ray Space Telescope and find that the $\gamma$-ray data are lagging the radio data by $300-400$ days. A possible explanation could be that the radio and the $\mathrm{GeV}$ data stem from different emission regions. We discuss our findings and propose that the detected jet precession can also account for the observed cavities in the X-ray emission on kpc-scales.
\end{abstract}

Keywords: radio galaxies; $3 \mathrm{C}$ 84; radio interferometry; VLBA; TeV emission

\section{Introduction}

3C 84 has long been an enigmatic example of an AGN. The physical nature of this particular AGN is of interest because of its very powerful radio jet. For some time it has been the brightest 
extragalactic radio source at high frequencies (e.g., [1]).The physical nature is also important with regard to understanding the relationship between the radio jet and the feedback in the cluster cooling flow (e.g., [2]). The classification of this AGN is still unclear. It has been classified as a broad emission line Seyfert galaxy, an obscured Seyfert galaxy, a narrow line radio galaxy, or a BL Lac object (for an overview please see [3]). Ref [3] showed that the ionizing continuum resembles that of a weak broad line Seyfert galaxy but with the hard ionizing continuum somewhat suppressed. They also found that the "innermost jet (within $0.1 \mathrm{pc}$ from the source) is best interpreted as a slightly off-angle BL Lac object jet".

\subsection{VLBI Observations of $3 C 84$}

The complex pc-scale radio structure of $3 \mathrm{C} 84$ has been investigated by many authors using VLBI observations at different observing frequencies (e.g., [4-9]). The radio structure of 3C 84 does not resemble the typical core-jet sources mapped with VLBI observations (e.g., [10]). The source structure in early VLBI observations can be seen in Figure 1 in an image taken from [11]. The morphology in the radio band can be nicely seen in the map by [12]. Highest resolution-observations with the RadioAstron space telescope resolved the edge-brightened radio jet down to only $30 \mu$ as from the core and revealed the position of the core [13]. The core is also named " $\mathrm{C} 1$ " in several papers.

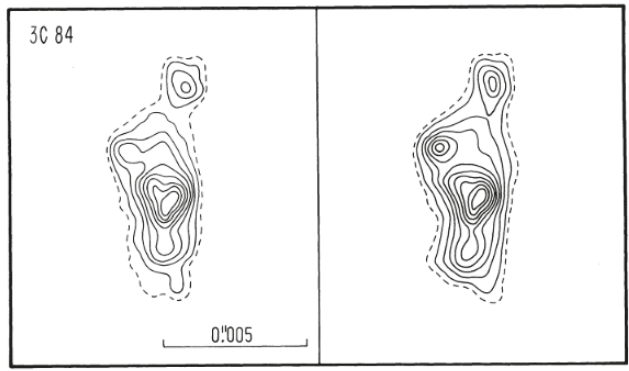

Figure 1. Two maps of 3C84 observed at $6 \mathrm{~cm}$ wavelength and taken from [11]. The map on the left shows the source structure in 1973.0 and the map on the right in 1974.1.

Nagai et al. [14] studied the sub-pc scale jet in 14 epochs of VLBI observations with the Japanese VLBI Network and the VLBI Exploration of Radio Astrometry (VERA) array between 2006 and 2009. They present evidence for a recurrence of jet activity and the emergence of a new component $\mathrm{C} 3$ associated with the radio outburst which started in 2005. Reference [15] perform a detailed analysis of the kinematics and light curve of the central sub-pc scale structure using archival VLBA data obtained at $43 \mathrm{GHz}$ and covering the period between January 2002 and November 2008. They find that C3 (relative to $\mathrm{C} 1$ ) shows moderate acceleration from $0.10 \mathrm{c}$ to $0.47 \mathrm{c}$. A very detailed investigation of the motion in 3C 84 has been performed by [5]. The authors use VERA at $22 \mathrm{GHz}$ and observed 3C 84 in 80 epochs between October 2007 and December 2013. They determine the averaged radial velocity of component $\mathrm{C} 3$ relative to the radio core to be $0.27 \pm 0.02 \mathrm{c}$. They explain the constant velocity of $\mathrm{C} 3$ as due to the advancing motion of the head of a mini-radio lobe. In addition, they find a non-linear component in the motion of $\mathrm{C} 3$ relative to the core. According to [5], C3 is the head of a radio lobe including hot spots at an early stage of the evolution. This finding is based on VLBA images obtained at $43 \mathrm{GHz}$ which show that $\mathrm{C} 3$ is embedded in a very complex structure [9].

\subsection{TeV Emission from $3 C 84$}

$\mathrm{TeV}$ emission from AGN is mostly found from blazars (e.g., [16]). Only six radio galaxies so far have been observed in the $\mathrm{TeV}$ regime (e.g., $[17,18]$ ) - $3 \mathrm{C} 84$ being one of them. While beaming is thought to play an important role with regard to the $\mathrm{TeV}$ energy production in blazars, the angle 
to the line of sight for radio galaxies seems to be unfavourable. The physical processes causing very-high-energy (VHE) radiation in radio galaxies are thus still unclear. There have been various attempts to investigate a possible connection and correlation between the radio emission sites and the $\mathrm{TeV}$ emission (e.g., for M 87: $[19,20])$. Several authors presented direct evidence that the $\mathrm{TeV}$ emission in the radio galaxy M87 originates from the core region [21-23]. The TeV emitting radio galaxy IC 310 is most likely a blazar (e.g., [24]) and HESS 0625-354 (e.g., [25]) as well.

In case of a blazar-like jet in the inner regions, $\mathrm{TeV}$ emission can be explained by Doppler boosting, caused by relativistic beaming of Inverse Compton (IC) scattered synchrotron radiation (e.g., [26-28]).

In the case of 3C 84, several authors have studied and discussed possible mechanisms that might explain the $\mathrm{TeV}$ emission. Reference [29] study multifrequency observations and modeled the overall spectral energy distribution (SED). They find that a single-zone synchrotron-self-Compton model, with an electron spectrum following a power-law with an exponential cutoff can explain the broadband SED and the multifrequency behavior of the source. This model, however, suggests an atypical low bulk-Lorentz factor or a velocity alignment closer to the line of sight than derived for the parsec-scale radio jet. Reference [30] study different scenarios to explain the fast gamma-ray variability. The authors prefer as the only plausible model the production of gamma rays in a magnetospheric gap around the central black hole. In addition, an enhancement of the magnetic field threading the hole from its equipartition value with the gas pressure in the accretion flow is required. The studies mentioned before encounter discrepancies.

We investigate 3C 84 to understand better the physical processes producing the $\mathrm{TeV}$ emission in this radio galaxy. Throughout the paper we adopt the following parameters: a luminosity distance $\mathrm{D}_{\mathrm{L}}=78.7 \mathrm{Mpc}$, at the source redshift of $\mathrm{z}=0.0176$ [31] with cosmological parameters corresponding to a $\Lambda$ CDM universe with $\Omega_{m}=0.308, \Omega_{\lambda}=0.692$, and $\mathrm{H}_{0}=67.8 \mathrm{~km} \mathrm{~s}^{-1} \mathrm{Mpc}^{-1}$ [32]. A proper motion of 1 mas yr$^{-1}$ corresponds to an apparent superluminal speed of $1.21 \mathrm{c}$, while 1 mas $=0.368 \mathrm{pc}$.

\section{Methods}

This manuscript relies on a re-analysis of observational data observed in the low energy (radio) and high energy (GeV-data) regime. In the following we describe the methods and tools used and developed in our data analysis.

\subsection{Re-Analysis of VLBA Data}

The MOJAVE ${ }^{1}$ (Monitoring Of Jets in Active galactic nuclei with VLBA Experiments) Program provides excellent VLBA monitoring data of pc-scale jets in AGN. We re-modeled 42 VLBA observations (15 GHz) of 3C 84 performed between 1999.99 and 2017.65. Gaussian circular components were fitted to the data to obtain the optimum set of parameters within the difmap-modelfit programme [33]. Every epoch was fitted independently from all the other epochs. Thus, the model-fitting procedure was performed blindly to not impose any specific outcome. We show the central structure of all the images with Gaussian model fit components superimposed in the Appendix A in Figures A1-A5.

Several authors (e.g., [4]) have mentioned that the well-known radio structure of $3 \mathrm{C} 84$ contains substructure. This substructure is usually not modeled. In our studies presented here, we also model the substructure to trace the details of the radio structural evolution better.

3C 84 is a very complex radio galaxy with large-scale jet emission on pc-scales. In addition, the source structure evolved significantly with time. The proper alignment of the individual components within the central region would require phase-referencing observations since the information about the absolute positions of the components is lost in the VLBI data reduction. However

1 https://www.physics.purdue.edu/MOJAVE/. 
and to our knowledge, phase referencing observations are not available for the time span analyzed in this manuscript. What we can measure, are relative offsets with regard to a chosen reference point.

We used a similar approach as described in [5]. The structure of the central region in 3C 84 changes significantly between 1999.99 and 2017.65. The core has only been observed and identified once in RadioAstron observations (in September 2013) [13]. We checked whether this core position would be detectable in all the epochs and could serve as a reference point. However, this core position could not be traced reliably across all the epochs and thus could not serve as a proper reference point. For a very crude alignment we used the separation between the larger lobe-structures (at about 10mas separation from the central structure) and the central part. Since the lobes were only detectable in a subset of the data sets, this provided only a coarse first step in the alignment of the maps.

Therefore we chose as the reference point the central position at epoch 1999.99 and kept this reference position through the epochs. This seemed to us the most promising and feasible approach to trace the structural changes in the central region as reliably as possible. By doing this, we did not impose any pre-knowledge and allowed an unambiguous analysis of the kinematics. Special care has been taken to correctly align the maps from epoch to epoch in the time sequence. The reference position (the core at $(x, y)=(0,0))$ has been chosen from the first epoch of the data we re-modeled and re-analyzed. This reference position has been kept by a very careful alignment of all the data from all the epochs and by checking the source evolution with time. The xy-positions of all jet components have been compared to the xy-positions of those same components that had been modeled in the epoch before and after. We aimed at minimizing the motion in $\mathrm{x}$ and $\mathrm{y}$ for each component across the epochs.

To summarize the basic procedure of our approach:

- We only use data from the MOJAVE survey to ensure comparing data obtained at the same frequency and similar quality of the data sets regarding the number of data points and the resolution of the observations.

- By fitting circular Gaussian components we model the details of the complex radio morphology and the substructure, which is usually not modeled due to the complexity of the source structure. Despite the complexity, our goal is to make robust component identifications which are not artifacts of the modeling procedure and then to follow and cross-identify these same components in the imaging time sequence. The model fitting has been performed blindly-each epoch has been modeled independently of other epochs. This enabled an unambiguous analysis of the kinematics.

- $\quad$ Since the "core" position determined in observations at $43 \mathrm{GHz}$ by Giovannini et al. [13] could not be identified unambiguously in all the epochs obtained at $15 \mathrm{GHz}$, we developed a "differential" alignment method to analyse the complex kinematics in 3C 84. To our knowledge, this differential analysis has not been applied in VLBI data analysis before.

- Within this differential alignment, the central position at epoch 1999.99 has been kept as reference position through the epochs. The deviation in xy-positions of all jet components across the epochs has been minimized.

Uncertainties of the modelfit component parameters were determined using bootstrap [34] For bootstrap applications to estimate uncertainties of VLBI results see [35]. We bootstrapped the adjusted residuals between self-calibrated interferometric visibilities and the best-fit difmap model. The residuals were first filtered from outliers, centered and distributions of the adjusted residuals were fitted using kernel density estimates (KDE). It was done independently for each baseline, correlation and frequency sub-band. We then added samples of the residuals from the fitted KDE to the model visibilities obtained from our best difmap model. The resulting bootstrapped visibility data sets were fitted in difmap using original best-fit model as the initial guess. It was done 300 times for each epoch. Thus we obtained a distribution for each parameter. The standard deviation was used for estimating the corresponding uncertainty. 


\subsection{Single-Dish Radio Flux-Densities}

For the study presented here, we make use of the light-curves provided by the University of Michigan Radio Observatory monitoring program (UMRAO) providing long-term single-dish radio observations taken at three frequencies $(4.8,8.0$, and $14.5 \mathrm{GHz})$. We combine the $14.5 \mathrm{GHz}$ data from the UMRAO with light-curve data obtained in $15 \mathrm{GHz}$ radio observations by the Owens Valley Radio Observatory $\left(\mathrm{OVRO}^{2}\right)$.

\subsection{Fermi-LAT Data Analysis}

We compare the light-curve evolution of $3 \mathrm{C} 84$ at low energies in the radio regime with high energy GeV data provided by the Fermi Gamma-ray Space Telescope (Fermi-LAT). To generate the $\mathrm{GeV}$ light curves we analyzed Pass 8 photon data ${ }^{3}$ using version v11r5p3 of the Fermi ScienceTools ${ }^{4}$, including all photon data within $15^{\circ}$ around the position of $3 \mathrm{C} 84$. We fit $3 \mathrm{C} 84$ with a log-parabola of the form

$$
\frac{\mathrm{d} N}{\mathrm{~d} E}=N_{0}\left(\frac{E}{E_{b}}\right)^{-\left(\alpha+\beta \log \left(E / E_{b}\right)\right)},
$$

with normalization factor $N_{0}, \alpha$, and $\beta$ left free for the fit, while the scale parameter $E_{b}$ was fixed to its catalog value. The parameters of sources with a separation less than $3^{\circ}$ from $3 \mathrm{C} 84$ where left free for the fit as well. All parameters of all sources between $3^{\circ}$ and $20^{\circ}$ where included in the analysis with their parameters fixed to their catalog values. Time epochs during which 3C84 was observed at a zenith angle greater than $90^{\circ}$ were excluded in order to avoid contamination from the Earth limb. The Galactic diffuse emission was modeled using gll_iem_v06.fits and the template iso_P9R2_SOURCE_V6_v06.txt. We performed an unbinned likelihood analysis, as described above, for every time bin of width 5 days in the interval MJD 54683-58428 (2008-08-05 until 2018-11-06).

In the following, we discuss the results of the re-analysis of the VLBA observations of the jet of 3C 84 (Section 3.1). We will present strong indication for precession based on the observations. We find some indication for a correlation between the appearance of the $\mathrm{TeV}$ flare and the phase of the precession. To check for further support of a precession, we apply a precession model to single-dish radio light-curves (UMRAO + OVRO data) to test whether the radio flux-density evolution is consistent with precession (Section 3.3). We compare the OVRO light-curve with $\mathrm{GeV}$ data obtained by Fermi and perform a correlation analysis (Section 3.4). We briefly discuss the different time-scales for precession found in observations at different wavelengths and spatial scales (Section 4.5). We discuss the indications for precession in 3 C 84 presented in this paper and in the literature. Finally we consider possible implications regarding cosmological questions (Section 4.6) and present our conclusions in Section 5.

\section{Results}

3C 84 reveals a complex radio structure on milli-arcsecond scales. In Figure 2a we show the radio structure visible in $15 \mathrm{GHz}$ observations with the VLBA. The central region reveals significant changes of the radio structure between 1999.99 and 2017.65 (for comparison please see a map from Jan. 2000 in Figure $2 \mathrm{~b}$ and from Apr. 2017 in Figure 2c. At the same time, epochs close in time to each other reveal comparable structure that confirms our modeling (see Figure 3). While the radio morphology in the central region consists of one curved chain of jet components in 1999.99, about $18 \mathrm{yr}$ later there are components next to each other in a much broader jet.

\footnotetext{
https://www.astro.caltech.edu/ovroblazars.

available from the Fermi-LAT data server at https://fermi.gsfc.nasa.gov/ssc/data/access/.

https://fermi.gsfc.nasa.gov/ssc/data/analysis/software/.
} 


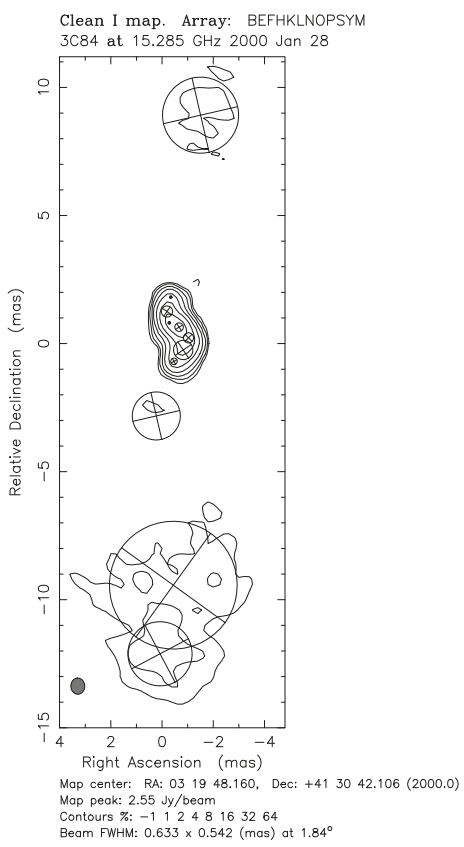

(a)

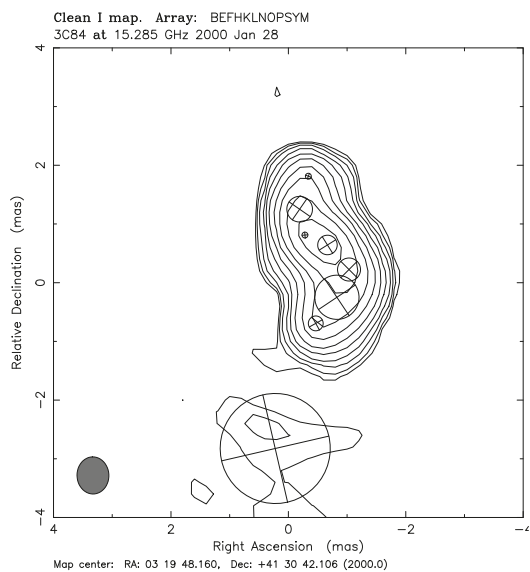

Wap center: RA: 0319 48.160, Dec: +41 30 42.106 (2000.0)

Contours \%: 0.61 .021 .732 .955 .018 .5214 .524

Contours \% 41.71 Fint $0.633 \times 0.542$ (mas) at $1.84^{\circ}$

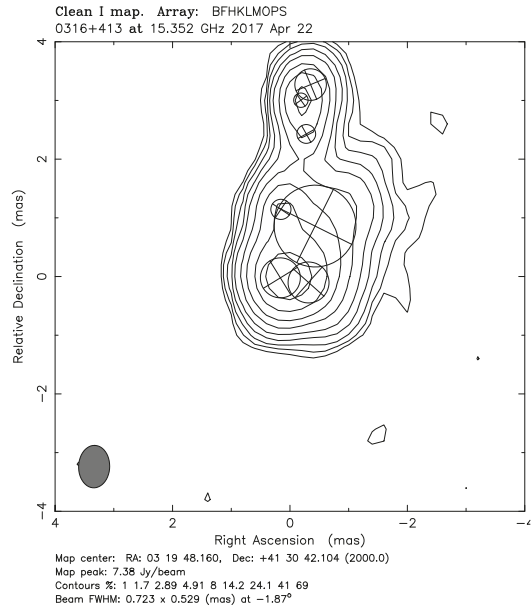

(c)

(b)
)

Figure 2. (a) A map of $3 C 84$ with the Gaussian components superimposed showing the structure in 15 GHz VLBA observations (January 2000). (b) a map of the central region of 3C 84 with the Gaussian components superimposed ((January 2000). (c) $17 \mathrm{yr}$ later the same central region is not one chain of components any more but the overall structure is broader as the morphology has evolved (April 2017). 


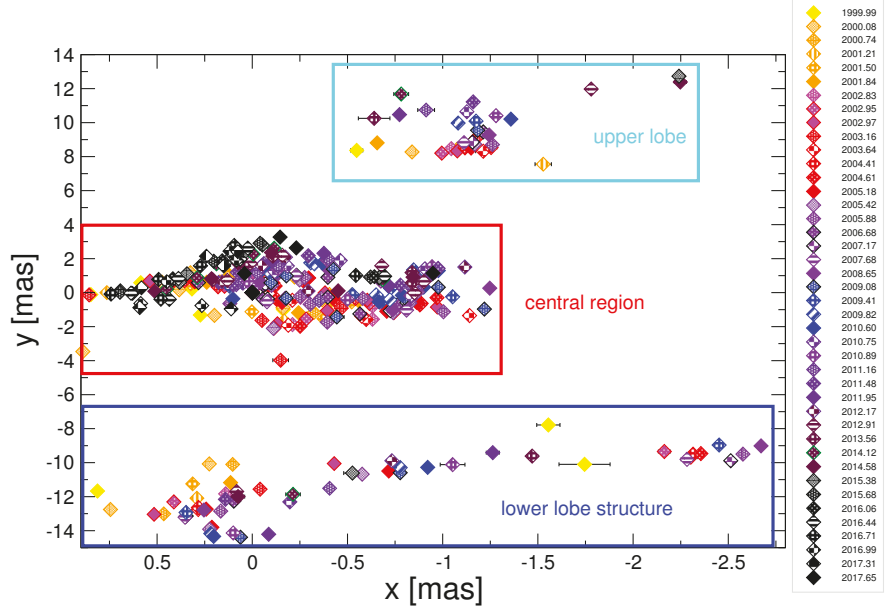

Figure 3. All the jet component positions of all the re-analyzed epochs are shown. We mark with a box (cyan) those components that belong to the upper lobe. The box in red marks the components that belong to the central region (zoom-in in Figure 4). The blue box marks the region with the lower lobe structure components.

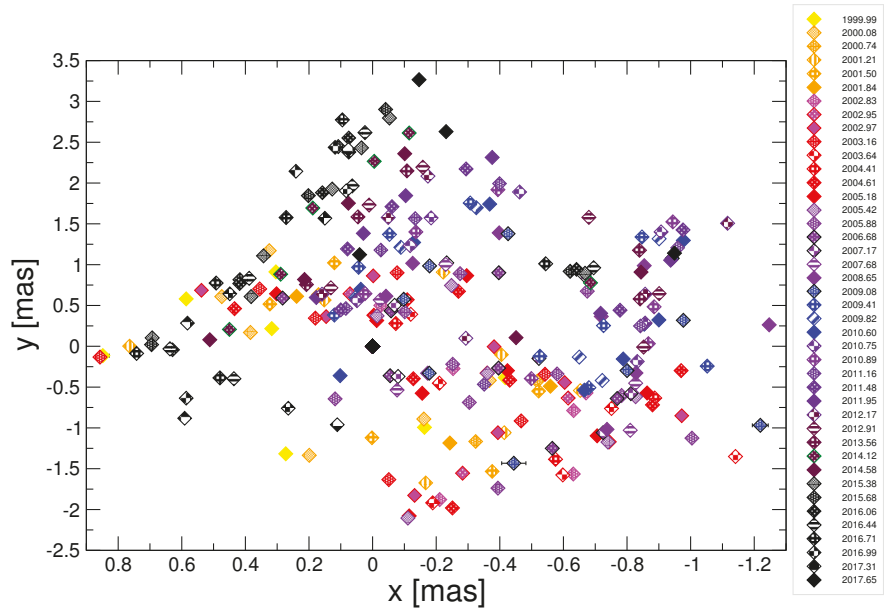

Figure 4. Jet components within the central region of 3C 84 between 1999.99 and 2017.65 (those within the red box in Figure 3.

\subsection{Evidence for Jet Precession Based on VLBA Data Analysis}

To determine the origin of these morphological changes, we studied the different parts of the pc-scale structure. In Figure 3 we show the positions of all the jet components which have been derived from the model-fitting of the VLBA data. The components are shown in an xy-plot and are displayed with their error bars. In most cases, the error bar is smaller than the symbol used to display the component. With boxes, we mark those components that will be discussed further within this paper. The red box marks the central region of $3 \mathrm{C} 84$, while the cyan box marks the jet features of the northern lobe. The components of the southern lobe are marked with the blue box.

In Figure 4 we show the xy-coordinates of the jet components which were detected within the inner region of 3C 84 (red box of Figure 3). The data points are displayed in 42 different colors and 
styles, marking the 42 epochs that were covered by the observations. The first epoch is shown in yellow. Darker colors mark the data from later years. With time, a displacement of the jet components relative to the early data is visible from this figure.

To trace the direction of the displacement, we calculate the arithmetic mean and uncertainty for each yearly interval of the 18 year period in Figure $5 \mathrm{a}$. In Figure $5 \mathrm{~b}$ we show the same relation but calculated based on two-year averaging. The "uncertainty" in both figures is a measure for the angular distribution of the jet components and their motion within the one year of binning. Obviously, the displacement follows a clear trend. The paths in Figure $5 \mathrm{a}, \mathrm{b}$ are indicated by green arrows. In addition, we performed a flux-density weighted averaging on yearly and bi-yearly timescale shown in Figure $5 c$,d respectively. The dominant trend of motion on a curve remains. The displacement with regard to the $x$-axis is similar as in the non flux-density weighted averaging. The main difference occurs with regard to the y-coordinate of the distribution. The flux-density weighted curve is constrained to a smaller range of (-0.3-0.6 mas) compared to (0.5-1) without flux-density weighting. At the end of this curved displacement (corresponding to the turn of the year 2016/17), the direction of motion seems to change (swings back). A similar motion and backswing is seen in the upper lobe in Figure 6.

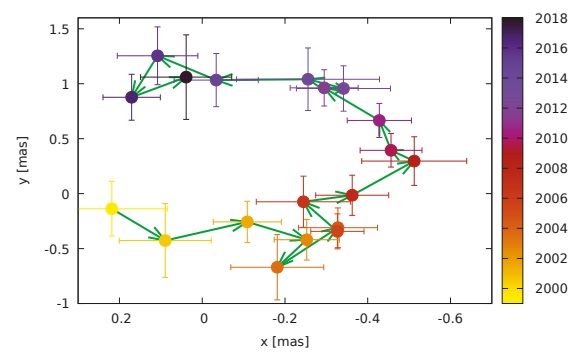

(a)

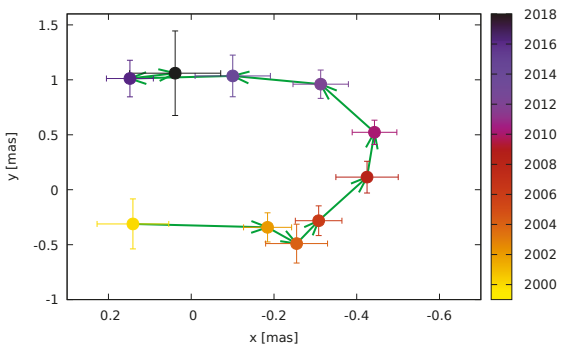

(c)

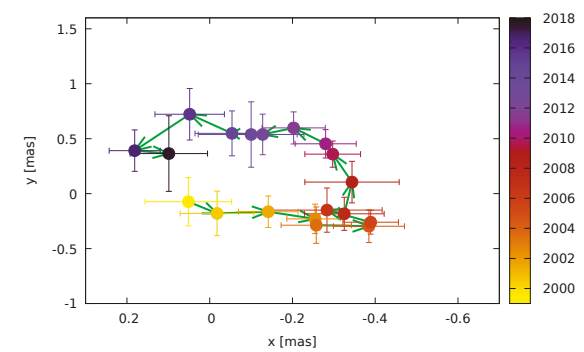

(b)

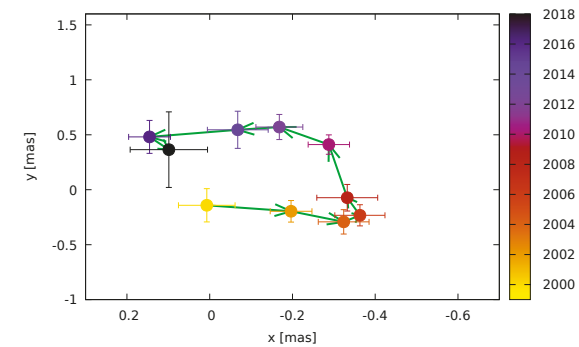

(d)

Figure 5. (a) Average values for the data in yearly intervals shown in Figure 4. To indicate the amount of source structure that moved, we show the uncertainties as well. The green arrows indicate the direction of the precessing motion. (b) The same relation as in (a) but averaged over two years in time. (c) Flux-density weighted average values in yearly intervals and averaged in two years in (d). The TeV flare observed by the MAGIC collaboration [30] occurs at the time of the projected reversal of the precessing motion-between the last two data points (dark blue and black). 


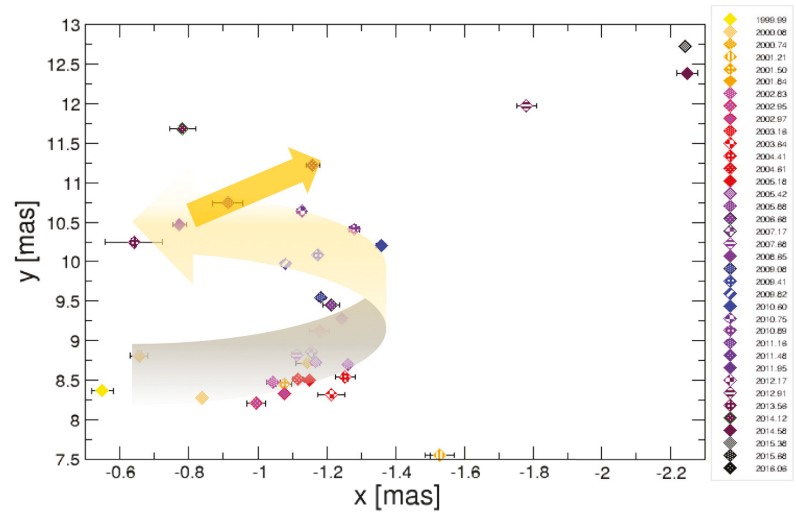

Figure 6. A zoom into the cyan box from Figure 3. An arrow is superimposed which indicates the general trend of motion (from grey to yellow). Again, there is some hint for the most recent data points to move "backwards" (indicated by the small orange arrow).

\subsection{VLBA Flux-Density Evolution of the Source Structure}

Figure 7 shows the flux-density of the upper lobe (cyan), the lower lobe (blue), the central region (red), and of the reference point/component (grey). The flux of the reference component is for most of the time period analyzed much brighter than the upper lobe or the lower lobe. Most flux is contained in the central region for the whole observing period. The flux-density of the central region reveals an increase starting around 2006-2007 and so does the flux-density of the reference component. Around 2017 , roughly $50 \%$ of the single-dish flux can be found within the reference component. At that time, the lobes are not detectable and the central region contains the total flux. We interpret the observed temporal and spatial structure of the jet evolution as clear evidence for a jet precession. In the following, we will provide further tests and in Section 4 we will discuss our findings and further arguments that this motion is consistent with precession.

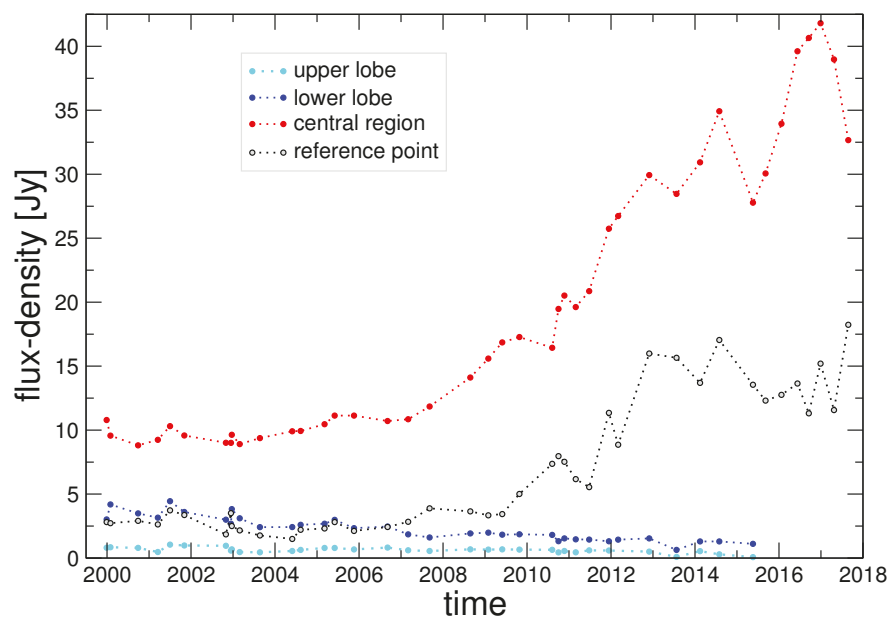

Figure 7. The flux-densities of the upper lobe, lower lobe, central region, and the reference component. The flux density of the central region is the brightest part of $3 \mathrm{C} 84$. 
The wealth of data observed for 3C 84 allows us to study the jet kinematics in great detail. This is, however, still work in progress, and the presentation of the full kinematic analysis is foreseen for a future paper.

\subsection{Radio Variability Consistent with Inner Jet Precession on Time-Scales of $\sim 100 \mathrm{yr}$}

The radio light curve based on the combined UMRAO (at $14.5 \mathrm{GHz}$ ) and OVRO data (monitoring with $40 \mathrm{~m}$ telescope at $15 \mathrm{GHz}$, Figure 8) shows first a gradual decrease from the peak at 1981.7 to the minimum at $\sim 2005$ and a subsequent gradual increase [6]. Such a gradual, coherent change in flux densities is qualitatively consistent with the precession of the jet bulk motion, where the underlying non-thermal continuum of the jet can generally be expressed as $S_{\text {jet }}(v) \propto v^{-\alpha}$ with $\alpha$ being the spectral index. The flux density is Doppler-boosted as the viewing angle decreases during the jet precession. The time-dependent flux density in the observer's frame can then be expressed as (see e.g., [36,37]),

$$
S_{\text {obs }}(t, v)=S_{\text {jet }}(v) \delta(\gamma, \phi)^{\xi}
$$

where $\delta(\gamma, \phi)$ is the time-dependent Doppler-boosting factor given by $\delta(\gamma, \phi)=[\gamma(1-\beta \cos \phi)]^{-1}$ with $\gamma$ being the Lorentz factor, $\beta$ the velocity of the moving jet components, and $\phi$ represents the angle with respect to the line of sight. The $\xi$ is the exponent of Doppler beaming and it is given by $\xi=p+\alpha$, where $p$ is the factor expressing the geometry of the emission region: $p=2$ corresponds to the case of a continuous, cylindrical jet emission, while $p=3$ expresses the contribution from discrete spherical components [38,39]; see also the original derivation of Doppler-beaming by [40,41].

We fit the composite function of the flux variability caused by the precessing jet, see Equation (2), to the combined radio light curve from UMRAO and OVRO monitoring. In total, we are searching for eight parameters- $S_{\text {jet }}, \xi, t_{\text {ref }}, P_{\text {prec }}, \gamma, \Omega, \phi_{0}, \eta_{0}$-where $t_{\text {ref }}$ is the reference epoch, $P_{\text {prec }}$ is the precession period in the observer's frame, $\Omega$ is the half-opening angle of the precession-cone axis, $\phi_{0}$ is the viewing angle of the precession-cone axis, and $\eta_{0}$ is the position angle of the precession axis on the sky plane (for a detailed description of the precession model see [37]). Since we have eight free parameters and we fit only the flux density evolution, the model is not constrained completely. However, at this stage, we are primarily interested in the qualitative assessment whether the radio variability is consistent with a realistic precession set-up. Further constraints on the model will come in combination with a detailed component analysis, as was previously done for OJ 287 [37].

In the fitting procedure, we make use of previous indications that the jet in $3 C 84$ should be precessing. This is based on the detection of colder bubbles or cavities that have different position angles with respect to the Perseus cluster core. They were detected as X-ray depressions in surface brightness maps [42]. Based on the position analysis and the estimates of the bubble buoyant rising time and their expansion speed, reference [43] constrained the half-opening angle of the precession cone, $\Omega_{\mathrm{DFS}}=50^{\circ}$, the viewing angle of the precession axis $\phi_{0}^{\mathrm{DFS}}=120^{\circ}$, and the position angle of the precession axis $\eta_{0}^{\mathrm{DFS}}=10^{\circ}$, and finally the jet precession period of $P_{\text {prec }}^{\mathrm{DFS}}=3.3 \times 10^{7} \mathrm{yr}$. We use these constraints to restrict the initial bounds of the fitted parameters shown in Table 1.

Based on only the radio light curve, the precession model can only be weakly constrained. However, the precession parameters obtained in this analysis can be used as initial values for a further analysis with the support of the kinematics of jet components, which is in preparation. That is why we decided, as a first approach, to limit the bounds of the basic precession angles based on the X-ray model and search for the best fit within these constraints, see Table 1 for a summary. Alternatively, one can fix the precession angles as well as the spectral index at specific values and search for the best solution for the other parameters. Concerning the precession period, we obtained two solutions as was already outlined and we summarize the best-fitted values in Table 1 . The solution with a longer period of $P_{\text {prec }} \simeq 88$ years (displayed in the top panel of Figure 8), with two different flux-density peaks, and the solution with a shorter period of $P_{\text {prec }} \simeq 40.5$ years with two equal flux-density peaks (lower panel of Figure 8). These two fits had a different upper bound for the searched precession period $t_{\max }$ 
in Table 1: $t_{\max }=200$ years for the obtained period of 88 years and $t_{\max }=50$ years for the obtained period of 40.5 years.

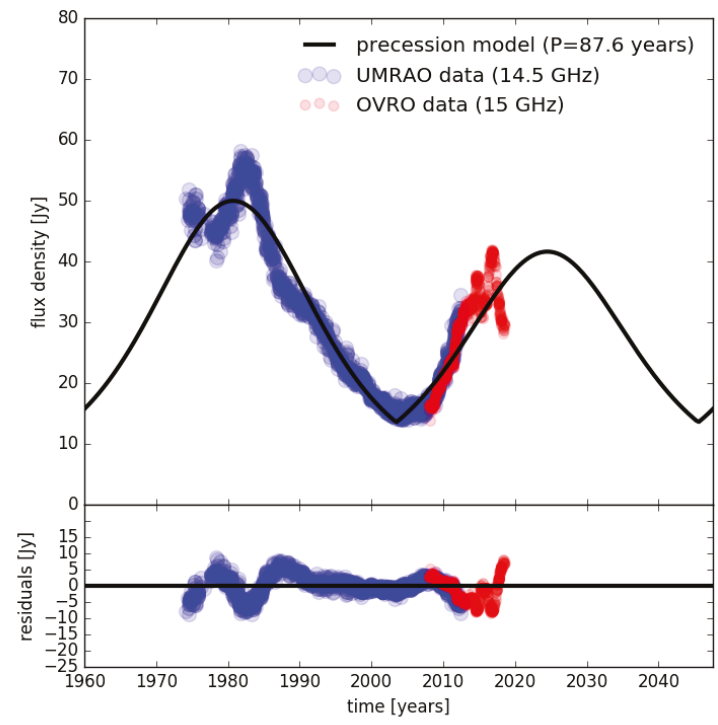

(a)

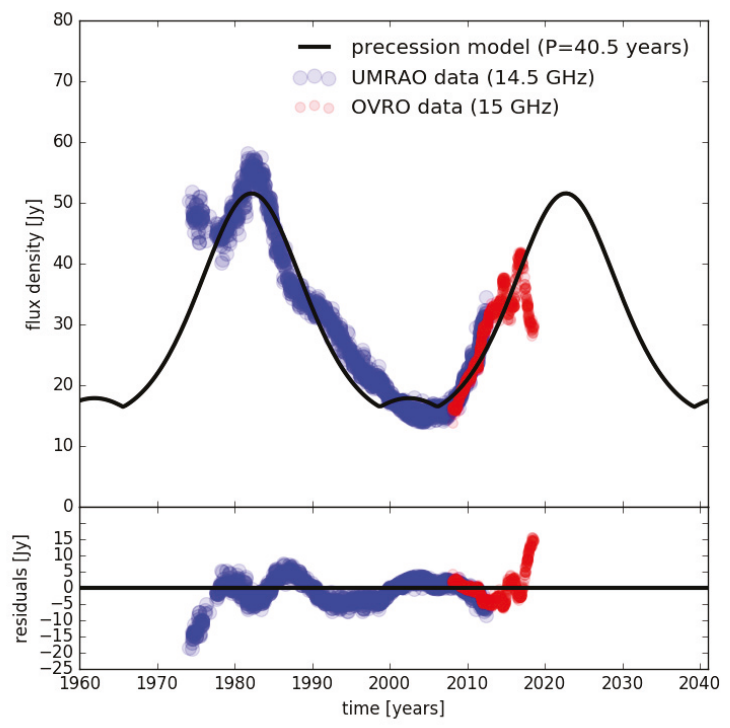

(b)

Figure 8. The jet precession model with the initial parameter bounds according to X-ray data, see also Table 1. (a) The model with two unequal flux-density peaks and the longer precession period of $\sim 88$ years. (b) The model with two equal flux-density peaks and the shorter precession period of $\sim 41$ years. The goodness of fit is nearly identical for the two models. 
Table 1. Table of fitted parameters in the precession model with the initial parameter bounds as indicated in the third column. The first column contains the parameter name, the second column lists the notation, the third one contains the initial bounds for the Levenberg-Marquardt algorithm [44,45], and the fourth and the fifth columns contain the fitted values for the longer and the shorter precession periods, respectively.

\begin{tabular}{ccccc}
\hline Parameter & Notation & Initial Bounds & $\boldsymbol{P}_{\text {prec }} \simeq \mathbf{8 8 ~ y r}$ & $\boldsymbol{P}_{\text {prec }} \simeq \mathbf{4 1} \mathbf{~ y r}$ \\
\hline Intrinsic jet flux density & $S_{\text {jet }}$ & $(1,100) \mathrm{Jy}$ & $17.8 \mathrm{Jy}$ & $19.3 \mathrm{Jy}$ \\
Exponent of Doppler boosting & $\xi$ & $(0,3)$ & $3.0 \pm 0.4$ & $3.0 \pm 0.6$ \\
Reference epoch & $t_{\text {ref }}$ & $(1900,2020) \mathrm{yr}$ & $1958.8 \pm 0.4 \mathrm{yr}$ & $1931.6 \pm 0.3$ \\
Precession period & $P_{\text {prec }}$ & $\left(1, t_{\text {max }}\right) \mathrm{yr}$ & $(88 \pm 1) \mathrm{yr}$ & $(40.5 \pm 0.2) \mathrm{yr}$ \\
Lorentz factor & $\gamma$ & $(1,20)$ & 1.09 & 1.05 \\
Half-opening angle of the precession cone & $\Omega$ & $\left(45^{\circ}, 55^{\circ}\right)$ & $55.0^{\circ}$ & $45.0^{\circ}$ \\
Viewing angle of the precession axis & $\phi_{0}$ & $\left(95^{\circ}, 130^{\circ}\right)$ & $95.0^{\circ}$ & $130^{\circ}$ \\
Position angle of the precession axis & $\eta_{0}$ & $\left(-30^{\circ}, 30^{\circ}\right)$ & $-6.1^{\circ}$ & $30.0^{\circ}$ \\
\hline
\end{tabular}

Afterwards, we broadened the initial bounds significantly to include all physically plausible values. The fitting procedure converged to the one with the precession period of $P_{\text {prec }} \simeq 101$ years. The best-fit values converged to values within the initial bounds, not at their limits as for the cases motivated by X-ray studies. Within the uncertainties, all the solutions can be considered consistent and can be further constrained by the future flux-density evolution.

In all the fitted cases, we obtain fit residuals with the maximum value of $\sim 15 \mathrm{Jy}$. These residuals could hint at a more complex kinematical model, for instance a smaller nutation-like motion as studied in the case of the blazar OJ287 [37]. The model, by default, assumes the constant intrinsic jet emission over the studied time interval $S_{\text {jet }}(v) \approx$ const, which, if variable, could be another source of variability in addition to the Doppler beaming effect.

The precession model with the initial bounds as listed in Table 1 converged with the precession periods of $P_{\text {prec }} \simeq 88 \pm 1 \mathrm{yr}$ and $P_{\text {prec }} \simeq 40.5 \pm 0.2 \mathrm{yr}$, respectively, the moderate Lorentz factor of $\gamma \simeq 1.1$, the base jet flux of $S_{\text {jet }} \simeq 18-19 \mathrm{Jy}$, and the Doppler boosting exponent of $\xi \simeq 3.0$, which implies the radio spectral index of $\alpha=1.0$ (continuous jet) or $\alpha=0$ (discreet jet) representing a steep or flat synchrotron spectrum, respectively. The precession angles are within the uncertainties consistent with the values inferred by [43]. We also tried the fitting procedure with the precession period within the interval $\left(10^{7}, 10^{8}\right) \mathrm{yr}$, but the fit did not converge. The precession period is approximately 90 years since the two flux-density peaks are not of the same amplitude- the earlier peak appears to be larger than the following one based on observed data, see Figure 8 (upper panel). In case the two peaks would be comparable, as may be refined by future radio observations, the precession period may be shortened by approximately a factor of two to approximately 40 years, as shown in Figure 8 (lower panel).

In addition, we analyze the evolution of the viewing angle for the three precession models with three different periods: 41,88 , and 101 years, see Figure 9 for the temporal evolution of the viewing angle, the temporal evolution of the apparent velocity, and the temporal evolution of the Doppler factor. Depending on the best-fit precession angles, the maximum viewing angle of the jet is close to $90^{\circ}$. The minimum viewing angle is for the solution with the shortest precession period of 41 years, $\phi_{\min } \sim 5$ degrees. This solution is also consistent with the viewing angle of $\sim 11$ degrees as inferred by Lister et al. [46] from the MOJAVE survey in the years 2002-2007. The precession period of 41 years is also preferred due to the temporal evolution of the apparent velocity which remains below $0.4 \mathrm{c}$ (in accordance with the results by $[5,6])$. According to this precession model, the viewing angle for the epoch 2019 should be $\sim 70^{\circ}$. 


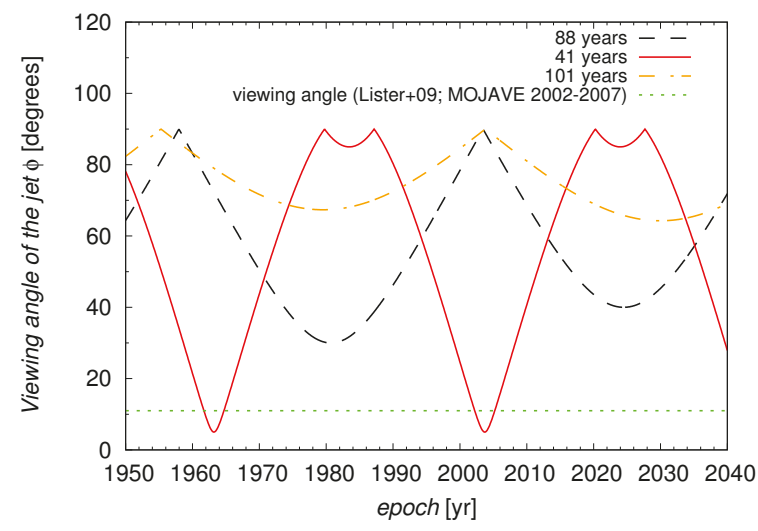

(a)

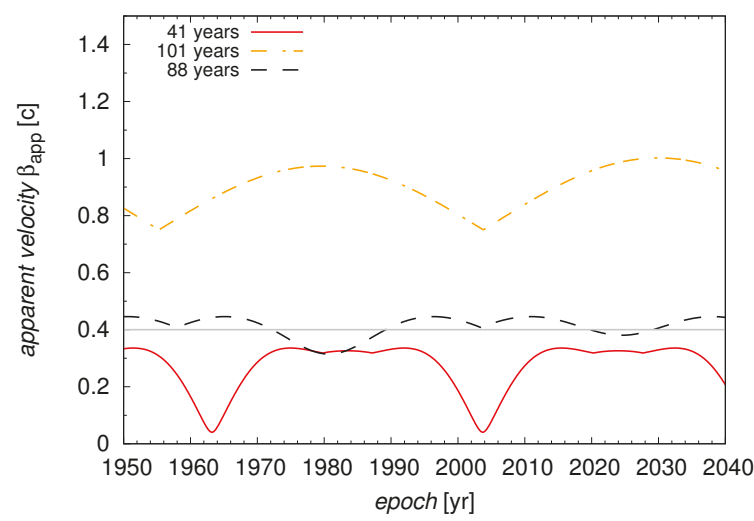

(b)

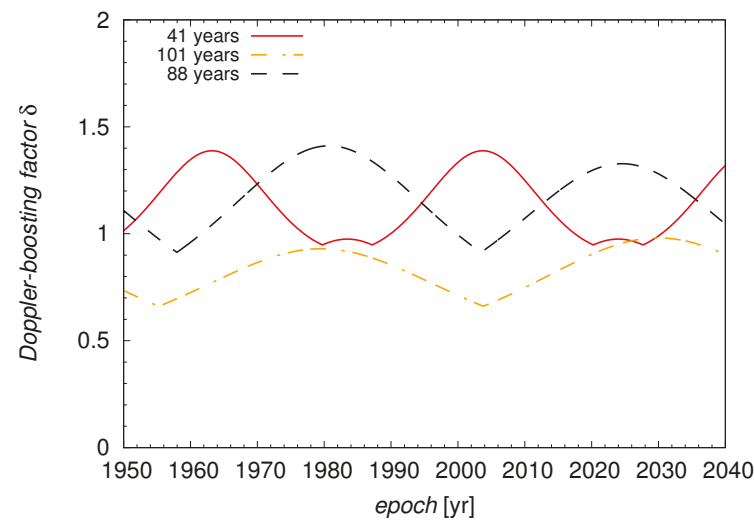

(c)

Figure 9. (a) Temporal evolution of the jet viewing angle $\phi(t)$ based on three precession models labelled according to different precession periods of 41,88 , and 101 years. The dotted horizontal line stands for the viewing angle inferred by Lister et al. [46] from the MOJAVE survey (2002-2007). (b) Temporal evolution of the apparent velocity which remains below $0.4 \mathrm{c}$ (for the precession model with $41 \mathrm{yr}$ ). (c) Temporal evolution of the Doppler factor which is less than 1.5 for all the precession models. 


\subsection{Correlation between Single-Dish Radio Flux-Density Data and Fermi Gamma-Ray Data}

A correlation analysis has been performed between the OVRO radio data and the $\mathrm{GeV}$ data observed by Fermi. For the Fermi data, 5-day binning was applied. The correlation analysis was performed in different ways. One approach similar to that described in [47] was used. In Figure 10b we show the normalized radio and $\mathrm{GeV}$ data, with the radio data shifted in time by +310 days (+meaning that the radio leads the $\mathrm{GeV}$ emission). Please note the different time axes for radio (upper axis) and $\mathrm{GeV}$ (lower axis). The data have been normalized by subtracting their average values and dividing them by their standard deviation. In addition, we applied the DCF routine by [48]. We find a moderate degree of correlation between the two light-curves with a peak value of $\sim 0.7$ (Figure 10c) (1.0 would be perfect correlation). We find a delay between 300 and 400 days and checked the significance of the DCF peak using $\mathbf{3 0 0 0}$ simulated noise-like light curves with the same probability density function (PDF) and power spectral density (PSD) as the original gamma- and radio light curves according to [49]. This method allows accounting for red-noise leakage and aliasing, see [49] for detail. Figure 10c shows the DCF and the range of DCF values that are not significant at a $3 \sigma$ level. We also fitted the gamma- and radio light curves with Gaussian Processes (GP) accounting for uncertainty in the parameters that determine the characteristic time scale and amplitude variation of the fitted function (i.e., hyperparameters). We applied Rational Quadratic kernel [50] that can account for different time scales of variability. An additional white noise kernel was used to describe the possible unaccounted noise. The posterior distribution of hyperparameters was obtained using the Marcov chain Monte Carlo (MCMC) method. For that purpose we applied an emcee sampler [51] and george Python library [52]. For the gamma-ray light curve data points with test statistics value "ts" $<25$ were filtered out. This corresponds to a threshold in a detection significance equal to 5 [53]. Using realizations of the fitted GPs with hyperparameters drawn from their posterior distribution (Figure 10f,g) it is straightforward to calculate the uncertainty of the time lag. Both ordinal CCF (on a fine regular grid interpolated by GP) and DCF were calculated. The result is that for ordinal CCF $68 \%$ the credible interval is $[-266,234] \mathrm{d}$ with median $-251 \mathrm{~d}$ (Figure $10 \mathrm{~d}$. For DCF the conservative $68 \%$ confidence interval is $[-509,-249] \mathrm{d}$ with median $-395 \mathrm{~d}$. The $\gamma$-rays are significantly delayed relative to the radio data. We discuss this finding in Section 4.

In the following, we briefly summarize the content of this section. We presented first details of our study of the long-term kinematic evolution of the central radio structure of $3 \mathrm{C} 84$. We find evidence for a smooth evolution with time which seems to be best described by precession. To our knowledge, this is the first time that such a continuous displacement has been reported. The appearance of the $\mathrm{TeV}$ flare reported by MAGIC seems to be correlated with a certain precession phase. In addition, we present the results of fitting a precession model to the radio flux-density data and show that a simple model fits the data. We derive a precession period in the range of $\sim 40-100 \mathrm{yrs}$, which depends on the characteristics of the precession model, and compare this to the much longer precession period derived earlier based on the observations of the X-ray cavities. A correlation analysis has been performed to determine the lag between the radio data (OVRO) and the GeV-data observed by the Fermi satellite. The $\mathrm{GeV}$-data lag the radio data by $300-400$ days. 


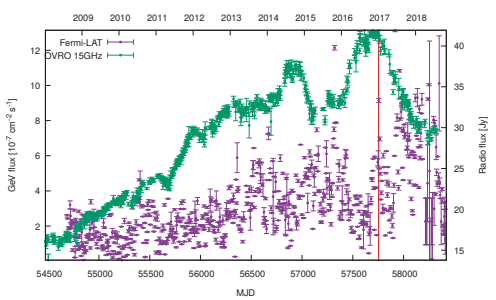

(a)

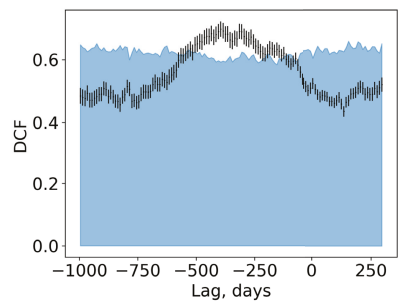

(c)

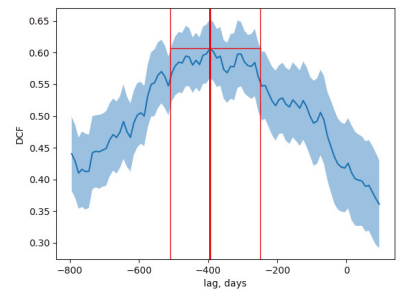

(e)

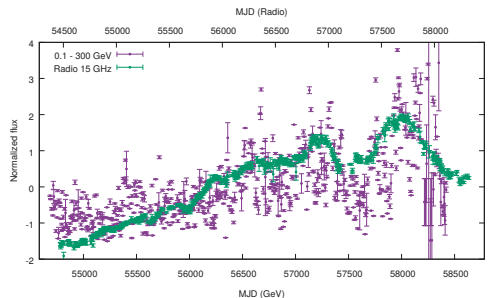

(b)

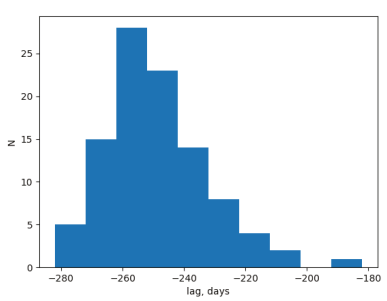

(d)

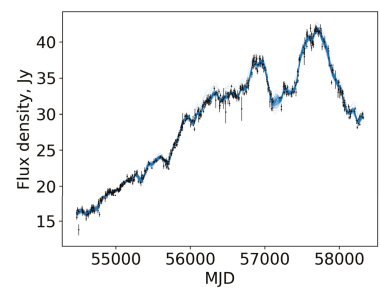

(f)

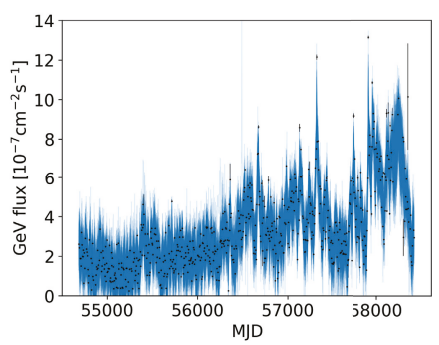

(g)

Figure 10. (a) OVRO monitoring data (green) and gamma-ray data from the Fermi satellite (violet). The red vertical line marks the time of the TeV flare [30]. (b) Superposition of the OVRO and Fermi data (mean-subtracted and time-shifted). (c) Significance analysis of the DCF results. The blue shaded region corresponds to a range of the possible DCF-peaks that occur due to a chance at $3 \sigma$ level. It was obtained using realizations of the noise-like light curves according to [49]. Black points and error bars shows the obtained DCF. (d) Distribution of the Cross-Correlation Function (CCF) peaks obtained using realizations of the Gaussian Processes fitted to the radio and gamma data. (e) Mean DCF and corresponding simultaneous $68 \%$-confidence band obtained using realizations of the Gaussian processes fitted to the original radio and gamma data sets. Red lines describe the procedure used to obtain the uncertainty of the DCF maximum. (c-e) show the results of a correlation analysis (based on the DCF-routine by [48]). (f,g) show the realizations of the fitted Gaussian Processes obtained from the posterior distribution of the hyperparameters for radio (f) and gamma (g) light-curves. 


\section{Discussion}

The radio structure of 3C 84 has long been a subject of debate. Reference [10] already discussed that the radio structure differs significantly from other objects mapped with VLBI. The main difference being that 3C 84 shows neither a linear structure nor the typical core-jet structure. The core has only recently been detected in high resolution observations with RadioAstron [13] within the central region.

Our detailed study of almost 18 yr of high-resolution observations of 3C 84 reveals essentially a smooth evolution of the radio structure. We do not find any evidence for dramatic changes in the outflow kinematics. Instead, it seems that the different appearance of the source structure is due to a change in the viewing angle-indicating that the source itself is moving or rotating with regard to the observer. As a consequence, different parts of the source structure become visible and detectable to the observer for different times of observation.

We therefore conclude, that the observed kinematic evolution provides a strong indication for precession of the central region (and upper lobe) in the pc-scale radio jet of 3C 84-based on a first analysis of the overall motion of all the jet features in 3C 84. Precession in 3C 84 has been claimed before by several authors based on simulations (e.g., $[43,54])$ to explain the Chandra observations of the X-ray cavities (e.g., [42]).

With the new results based on the long-term kinematics of the pc-scale jet, observational support for the precession origin is provided. The precessing motion of the jet features also quite naturally explains the long-term flux-density variations seen in the radio. Independent support for a scenario with a varying viewing angle (thus supporting the precession model) comes from the detection of a change in the $\gamma$-ray spectral properties for which variations in the Lorentz factor and/or the viewing angle are required to explain the observed variability from February 2011 onwards [55].

\subsection{Origin of Radio Variability in 3C 84: Variable Jet Activity or Variable Doppler Beaming?}

Several authors discuss the physical origin of the long-term (decades) radio variability in $3 \mathrm{C}$ 84 (e.g., [14]). The standard interpretation so far is that this variability is due to a variation of the jet activity or even restart of the jet activity. This interpretation is also based on the presence of the multiple radio-lobe structures on 10 mas scale. The double lobes on the 10 mas scale might originate in the 1959 outburst [56]. Nagai et al. [9] link the emergence of C3 with the outburst starting around 2005. C3 has also been identified as a hotspot of the innermost radio structure [9]. The understanding of these authors is, that the lobes account for most of the radio flux at centimeter wavelengths which is not correct, as we showed in Figure 7. The flux-density is dominated by the flux of the central region and the reference component contributes about $50 \%$ of the total flux. The general understanding is that the lobes are decelerated components. According to this standard interpretation, 3C 84 should have experienced several outbursts in its jet activity which lead to the formation of these lobes. Based on this argumentation, the jet power is variable on the timescale of decades or longer. The long-term radio light curve would then be explained by the variable jet activity alone. This standard interpretation takes only the variations of the intrinsic jet activity into account but not changes due to a varying Doppler factor that arise due to the bulk motion of the jet.

However, based on the data and analysis presented in this paper, we argue that the long-term radio variability is most likely due to the changes in the Doppler factor $\delta$. We further illustrate this in Figure 11 where we plot the normalized Doppler factor $\delta_{\text {norm }}=\delta_{\max } / \delta_{\min }$ as a function of the precession phase for the three different precession solutions (see Tables 1 and 2 for details). The ratio of the maximum to the minimum Doppler factor is relatively small $\sim 1.4-1.5$, but it is sufficient to account for the flux variability, with the variability amplitude given by $A=S_{\max } / S_{\min }=\left(\delta_{\max } / \delta_{\min }\right)^{p+\alpha}$. We do not exclude that the intrinsic jet activity is variable in addition and that the outer lobes might have been created in outbursts. But the current radio variability on the timescale of 10 years seems to be related to and caused by changes in the Doppler beaming and can be modeled as such. Our reasoning is based on the kinematics presented in this paper, the radio variability which can be explained by the precession model, the additional evidence for precession discussed and modeled for the X-ray 
emission, and to the SED modeling (discussed later in this paper) which requires a change in the angle to the line of sight.

Table 2. Table of fitted parameters in the precession model with the broadened initial parameter bounds. The first column contains the parameter name, the second column lists the notation, the third one contains the initial bounds for the Levenberg-Marquardt algorithm, and the fourth column contains the fitted values.

\begin{tabular}{cccc}
\hline Parameter & Notation & Initial Bounds & Fitted Value \\
\hline Intrinsic jet flux density & $S_{\text {jet }}$ & $(1,100) \mathrm{Jy}$ & $64.7 \mathrm{Jy}$ \\
Exponent of Doppler boosting & $\xi$ & $(0,5)$ & $3.8 \pm 0.7$ \\
Reference epoch & $t_{\text {ref }}$ & $(1900,2020) \mathrm{yr}$ & $1954.3 \pm 0.7 \mathrm{yr}$ \\
Precession period & $P_{\text {prec }}$ & $(1,200) \mathrm{yr}$ & $(101 \pm 2) \mathrm{yr}$ \\
Lorentz factor & $\gamma$ & $(1,20)$ & 1.5 \\
Half-opening angle of the precession cone & $\Omega$ & $\left(5^{\circ}, 90^{\circ}\right)$ & $24.2^{\circ}$ \\
Viewing angle of the precession axis & $\phi_{0}$ & $\left(0^{\circ}, 180^{\circ}\right)$ & $88.5^{\circ}$ \\
Position angle of the precession axis & $\eta_{0}$ & $\left(-180^{\circ}, 180^{\circ}\right)$ & $54.5^{\circ}$ \\
\hline
\end{tabular}

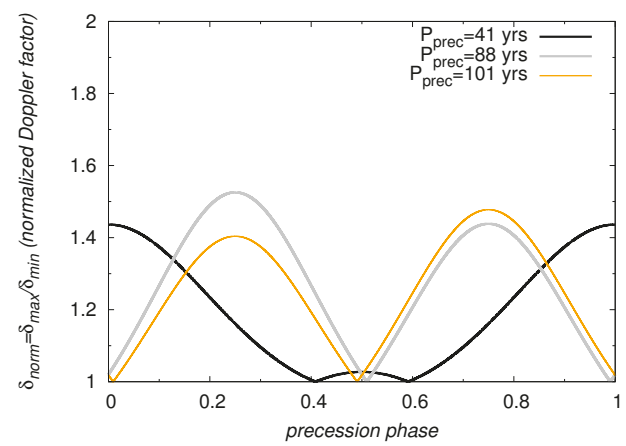

Figure 11. The normalized Doppler-beaming factor $\delta_{\text {norm }}=\delta_{\max } / \delta_{\min }$ as a function of the precession phase for three different precession solutions indicated in the key according to the precession period (see Tables 1 and 2 for details).

\subsection{Possible Causes of the Jet Precession in $3 C 84$}

Two possible scenarios are usually invoked to explain the precessing motion (see also discussion by [43]):

1 A binary black hole at the center of the NGC 1275 galaxy, which would cause a precession of the primary black hole disc [57] is the first and most often suggested explanation.

2 An instability in the disc that could warp the disc and cause the precession [58].

Independent support for a binary origin of the precession comes from optical observations of 3C 84. For example, [59] have identified two spiral galaxy systems in the host galaxy of 3C 84 and suggested that they are two colliding galaxies. Note however, that [60] have shown that one of the two systems is probably an unrelated spiral galaxy seen in projection against NGC 1275. Detailed observations of NGC 1275 with the HST reveal an ongoing collision between a spiral system seen nearly edge-on and a giant elliptical with peculiar faint spiral structure in its nucleus [61], indicating that both galaxies are colliding at over $2700 \mathrm{~km} / \mathrm{s}$. Theoretical modeling by [62] provides a general explanation of jet precession as the result of the merger of two galaxies.

In a subsequent paper we will present a more detailed analysis of the overall kinematics, hopefully being able to place constraints on the binary black hole system parameters of the system. 


\subsection{GeV-Emission from 3C 84}

Previous studies suggested that the increase in the $\gamma$-ray flux may be correlated with the radio flux densities in 3 C $84[6,63]$. We study in Section 3.4 a possible correlation between the $\mathrm{GeV}$ light-curve obtained with Fermi and the radio light-curve observed within the OVRO monitoring program including the most recent data (see Figure 10a-c). We confirm the earlier findings of a correlated variability between the high energies $(\mathrm{GeV})$ and low energies $(15 \mathrm{GHz})$. In addition we find that the $\gamma$-ray data lag the radio data by about 300-400 days. It is usually assumed in models of fast particle acceleration (e.g., [64]) that the relation is the opposite: the radio data lag the high-frequency data. Fuhrmann et al. [65] performed a cross-correlation analysis between radio and $\gamma$-ray light-curves of 54 Fermi-bright blazars based on a source stacking analysis. They found an evidence for the radio data lagging the $\gamma$-rays when averaging over the whole sample. Reference [66] performed a cross-correlation analysis using discrete correlation functions between the radio and the $\gamma$-ray light curves. They found 26 sources with significant correlations where in most of the sources the $\gamma$-ray peaks lead the radio.However, this relation seems not to be consistently present even for blazars, as a study shows [67]. The results of their investigation show that correlations between radio and gamma-ray light curves of blazars are only found in a minority of the sources (over a 4 yr period). The conclusion of this study is that most likely the connection between both frequency regimes is of more complex nature. Currently there is no accepted explanation why the gamma-ray data lag the radio data by a significant amount of time in $3 C 84$. We speculate that the lag could be related to the precession reported in this current paper and the geometry of the system, e.g., the radio emission and gamma-ray emission might be produced in different regions of the jet. Due to the precessing motion, these regions would be detectable at different times.

3C 84 shows a significant brightness increase in the radio since 2005 [68]. The maximum of this brightness increase seems to be reached in 2017 (Figure 10). The $\gamma$-ray flux has steadily increased between 2008 and 2016. For the period between 2013 and 2015, a correlated brightening of the nucleus in the X-rays and $\gamma$-ray regime has been reported [69]. Between 2016 and 2017 a dip in the $\gamma$-ray flux is observed, which seems to follow a similar dip seen before in the radio light-curve. The $\gamma$-ray flux of 3C 84 is known to be highly variable on short ( days to weeks) time-scales (e.g., [55]).

\subsection{Potential Origin of the TeV Emission in 3C 84}

3C 84 was detected in two observational campaigns by MAGIC between 2009 October and 2010 February and between 2010 August and 2011 February and by VERITAS in ToO observations between 2012 December and 2013 February as well as between 2013 October and November [29,70,71]. A bright TeV flare has been observed in the night from 2016 December 31 to 2017 January 1 by [30]. The MAGIC collaboration has investigated different models to explain the $\mathrm{TeV}$ emission observed in $3 C$ 84. According to these authors, the only mechanism to account for the observed luminosities in the brightest $\mathrm{TeV}$ flare is the generation of gamma-rays in a magnetospheric gap around the central black hole. In addition, an enhancement of the magnetic field threading the black hole from its equipartition value with the gas pressure in the accretion flow is required. This explanation refers to the brightest TeV-flare. In the following, we report on results from previous studies with regard to lower-level emission. The TeV emission observed in 3C 84 before the 2016/17 event has been modeled by several authors (e.g., $[55,69])$ within a one-zone synchrotron self-Compton model. A change in the spectral behavior in the LAT energy band has been found around 2011 February [55]. The authors suggest that at earlier times the flux changes were caused by high-energy electron injections into the jet. After February 2011, the $\gamma$-ray flares were either caused by a changing Doppler factor due to variations of the jet Lorentz factor and/or the angle to our line of sight. A precessing system leads to changes of the angle to the line of sight.

The studies by $[55,69]$ point towards a change in the $\gamma$-ray spectral properties which can be explained (after February 2011) by a changing Lorentz factor and/or the viewing angle. This finding supports our result of detecting precession for 3C 84 . 
Interestingly, we find that the time of the $\mathrm{TeV}$ flare seems to correlate with the (projected) reversal point of the precessing motion of the components. The flaring activity appears to have occurred over only several days. Ahnen et al. [72] report on MAGIC telescope observations at very-high energies for a total of $253 \mathrm{~h}$ from 2009 to 2014 and no flares seem to have occurred during this time. Also, the complete data observed for the Perseus cluster by MAGIC between 2009 and 2017 are presented by [73]. It seems that 3C 84 flared only once at TeV-energies. Our study and correlation analysis will have to be continued in the future with more data.

In summary, we don't know what the specific physical conditions at this position are, or whether the flare is caused by intrinsic or environmental effects, or by a geometric effect. It is possible, that the sites producing the radio, $\mathrm{GeV}$, and $\mathrm{TeV}$ emission in $3 \mathrm{C} 84$ are not cospatial. As already mentioned, the precession has been invoked by simulations based on the X-ray data to explain the cavities. As [74] have confirmed based on 900 ks Chandra X-ray observations, there is a strong hard X-ray excess within the central region of the Perseus cluster. We suggest, that the precessing motion of the radio jet might lead to an interaction with ambient $\mathrm{X}$-ray gas. This could explain the TeV-flare. Especially, since the $\mathrm{TeV}$-flare seems to correlate with the projected turning point of the precessing motion. Another possibility, which we will explore with the detailed kinematic analysis planned for a future paper, whether the TeV-flare can be related to specific jet component behaviour. However, we may conclude that the origin of the $\mathrm{TeV}$ emission in $3 \mathrm{C} 84 \mathrm{might}$ be related to the jet precession. We assume- this will have to be confirmed by modeling - that the source is pointing towards us with a smaller viewing angle at that time. Further support for our findings stems from the studies of [3] who suggest that the innermost jet resembles a slightly off-angle BL Lac object.

\subsection{The Derived Time-Scales for Precession in 3C 84}

With this paper, we provide strong indication for a precessing jet in $3 \mathrm{C} 84$ based on almost 18 years of VLBA observations. This finding is based on the highest resolution observations discussed for this source in this paper. This is a first analysis of the overall kinematics and most likely can be improved by studying the detailed individual component motions.

\subsubsection{Time-Scales for Precession in 3C 84: VLBI Maps}

In case, precession plays an important role for the source structure of $3 \mathrm{C} 84$, then older maps should reveal a similar source structure as some of the maps we show in this manuscript. We searched the archives for earlier maps of 3C84. In Figure 1 we show two maps obtained in $6 \mathrm{~cm}$ VLBI observations in 1973 (left) and 1974.1 (right). Both reveal a central structure which seems to consist of two features. This is similar to what we find around the time of 2014 and later (see Figures A4). Although the observation from 1973 and 1974.1 have been performed at a lower frequency, the basic central structure looks similar. This finding provides an estimate for the precession time-scale of the order of about 40 years.

\subsubsection{Time-Scales for Precession in 3C 84: Single-Dish Radio Flux Modeling}

To check for consistency of the finding of precession based on the source kinematics, we fitted a precession model to the so far available single dish radio observations (at 14.5 and $15 \mathrm{GHz}$ ). Indeed, a precession model nicely reproduces the observed flux-density evolution and thus provides further support for the precessing motion deduced based on the VLBA jet observations. Although we made use of all radio data available since the beginning of UMRAO-monitoring, it is clear from Figure 8 that we can not cover several cycles. This fit supports our finding from the morphological studies of the jet but can not be regarded as a firm proof of the precession model. Several precession cycles will have to be observed in order to establish the precession unambiguously. This, however, will most likely be the work for future generations of astronomers given the precession period of $\sim 88-101 \mathrm{yrs}$. As discussed before, a value of $\sim 41 \mathrm{yrs}$ is also possible, which could be tested in the near future. Based on the VLBA data analysis of the kinematics of $3 C 84$ presented in this paper, the precession time-scale seems 
to be shorter. From Figure $5 b$ it seems that one half of an orbit (blue arrow from about 2007-2017) takes about $10 \mathrm{yr}$. This VLBI precession period seems to be inconsistent with the precession fit to the single-dish light curve. This inconsistency can be softened by considering the time after 2010 when the radio curve rose more rapidly. From Figure 8 this seems to fit the OVRO data light-curve. This might imply a change in the value of the precession period.

\subsubsection{Time-Scales for Precession in 3C 84: X-Ray Cavity Simulations}

Additional support for the precession model comes from the SED-modeling of the multi-wavelength observations [55] and the simulations reproducing the X-ray cavities (e.g., [43,54]). Several groups have nicely shown that precession is capable of reproducing the $\mathrm{X}$-ray observations. The modeling of the required precession based on the X-ray cavities leads to a different value for the precession time-scale.

The apparent discrepancy between the precession time-scale of the inner jet, $P_{\text {prec }} \simeq 40-90 \mathrm{yrs}$ based on our analysis of the kinematics, and the precession period based on the cavities, $P_{\text {prec }}^{\text {DFS }}=3.3 \times 10^{7} \mathrm{yr}$, implies a difference of five orders of magnitude. This difference can be explained by one of the three following effects or by their combination,

- The longer precession period is based on the X-ray observations of larger, kpc structures where cavities or bubbles can be resolved. These structures come from older times, when the supermassive black holes of the merging two galaxies were further apart from each other. The shorter precession period is related to the inner jet, which reflects the more recent activity. In the meantime, the two black holes could have approached significantly. Typical orbital decay time-scales from modelling are $10^{7}-10^{9} \mathrm{yr}$ for gas dynamical friction (e.g., [75]). The shortening of the precession period depends on the merging time-scale of the binary system of $3 \mathrm{C} 84$.

- The X-ray cavities could be created only occassionally during epochs of increased intrinsic jet emission $S_{\text {jet }}$, which can be time-variable. The sparse ejection of rising bubbles, i.e., not during every precession period, would prolong the periodicity in case the precession period determination is based on the resolved pair of bubbles. In other words, smaller bubbles could be beyond the detection threshold, or more bubbles could be blended into an apparent larger one.

- Faster precession motion may be superposed on the slower motion. Faster precession on the time-scales of $\sim 100 \mathrm{yr}$ could be driven by a secondary black hole, while the slower precession could be due to the warped disc on larger scales, which leads to the misalignment of angular momenta and the Lense-Thirring precession of either the inner portion of the disc and the black hole [76] or the precession of an outer disc [54], which can affect the jet kinematics depending on where it originates (Blandford-Znajek [77] or Blandford-Payne [78] process).

Dunn et al. [43] already discuss whether the different pairs of X-ray cavities/bubbles belong to different "generations" and whether the precession is speeding up. A speeding up would explain a decrease in the precession time scale. It seems likely that the precession angle is changing (from kpc-scales to pc-scales). However, in the current modeling presented in this paper, the precession angle is kept fixed to get a first rough idea on the parameters of the system. We plan to take care of this point in the future kinematic study. More data and simulations are clearly needed to answer this question.

We want to stress, that the multi-wavelength observations performed with different telescopes providing different resolutions all seem to come to the same conclusion, that precession seems to play a dominant role in explaining the different physical phenomena which make 3C 84 so special. It is not really a surprise that the shortest time-scale is found based on the highest resolution observations (radio). This would have been expected since these observations probe the region of the most recent and youngest activity. In case the precession model is correct and originates in a binary black hole scenario of approaching black holes, the orbital evolution of the binary system should lead to changing precession time-scales with time. 
Of course, much more data and detailed modeling of those is required to connect the different spatial scales and the proper time-scales of precession potentially at work in $3 \mathrm{C} 84$ to finally prove the precession model and decode the dynamics of this source.

\subsection{Implications for Cosmology}

With this paper we present another important piece to understand the relation between galaxy mergers and the triggering of AGN jet activity. It has long been derived (by analysis and numerical modeling) that mergers of galaxies enable the removal of angular momentum and the transport of material to the central engine within the hierarchical galaxy formation model (e.g., $[79,80])$. The collision of galaxies can lead to the formation of binary black hole systems (e.g., [80]). The so-called final parsec problem [81] results from an obvious conflict between the observational finding that most supermassive binary black holes (SMBBH) in nearby galaxies seem to have coalesced quickly and the theoretical expectation of a stall on a time-scale longer than the Hubble time. Unbound AGN binary systems with separations of the order of kiloparsecs have been found (e.g., [82]). Many authors have presented indirect evidence for supermassive binary black holes based on pc-scale jet precession (e.g., OJ287: [37,38]; 1928+738: [83]; 3C 454.3: [84]; 3C 120: [36]; PG 1553+113: [85]).

NGC 1275-as Hubble observations show-is an ongoing merger of two spiral systems and 3C 84 seems to be precessing. This latter finding provides further strong support for the more general hypothesis that AGN activity is related to galaxy mergers and the consequent formation of supermassive black hole pairs (e.g., [86-88]). Precession is a fingerprint to discover these systems. Precession is also a tool to derive information on the physical parameters of the binary system, e.g., as was done for OJ 287 [37], for the other sources by the authors listed at the end of the previous paragraph and for many more sources. X-ray cavities in addition provide an independent proof for the validity of this concept and can help to study the long-time cosmic evolution of these systems.

More: In case our interpretation is correct and the flux-density evolution is due to precession, then the claim of restarting activity [14] is probably not corret.

\section{Conclusions}

By re-modeling and re-analyzing 42 epochs of $15 \mathrm{GHz}$ VLBA observations of 3C 84, we find evidence for a long-term continuous displacement of the central radio structure. This long-term variation has not been shown before and can best be explained by precession. We thus find clear indication for precession of the jet source in $3 \mathrm{C} 84$. The derived precession period (based on fitting the UMRAO and OVRO radio data) is in the range of $\sim 41-101$ yrs. A comparison with maps of $3 \mathrm{C}$ 84 taken in 1973 and 1974.1 which show a similar central readio structure support a value of $41 \mathrm{yrs}$ for the precession period. The derived values for the apparent speeds also seem to favor a precession period of abour 40 yrs. This value differs from the precession period derived earlier to explain the $\mathrm{X}$-ray cavities, which is five orders of magnitude larger. We discuss several possible explanations. The most plausible reason to explain the difference is the evolution of the merging process of a binary black hole system. In this paper, we study the evolution of the inner jet, while the X-ray cavities most likely provide information about an earlier phase of the approaching black holes. $3 \mathrm{C} 84$ seems to show a change in the precession period with time-this result and its implications should be studied in more detail.

To our knowledge, this is the first time that observational evidence for precession is presented in $3 \mathrm{C} 84$. We tentatively suggest that the $\mathrm{TeV}$-flaring coincides with a specific precession phase. Our results indicate that the dynamical evolution of the jet (and the beaming factor) might play a role in explaining the $\mathrm{TeV}$ emission in this source. SED-modeling of multi-frequency data (e.g., $[55,69]$ support our findings that the angle to the line of sight is changing. Beaming thus plays a role in this AGN where the innermost jet resembles a slightly off-angle BL Lac object [3]. The accordance of the direction of the precession with the sizes and shapes of the X-ray cavities supports our claims of the reality of the three-dimensional motion of the radio jet in $3 \mathrm{C} 84$. 
Author Contributions: S.B. prepared most of the text in collaboration with C.F. and M.Z. S.B. performed the VLBA data re-analysis. M.Z. provided the precession analysis. The Fermi-LAT Data Analysis was done by F.J. The correlation analysis was contributed by M.Z., I.P., and F.J. UMRAO data were provided by M.A. and H.A. All co-authors contributed in the discussion of the presented results.

Funding: M.Z. acknowledges the support by the National Science Centre, Poland, grant No. 2017/26/A/ST9/00756 (Maestro 9). This research has made use of data from the OVRO 40-m monitoring program [89] which is supported in part by NASA grants NNX08AW31G, NNX11A043G, and NNX14AQ89G and NSF grants AST-0808050 and AST-1109911. This research has made use of data from the University of Michigan Radio Astronomy Observatory which has been supported by the University of Michigan and by a series of grants from the National Science Foundation, most recently AST-0607523 and by NASA Fermi GI grants.

Acknowledgments: We thank M. Böttcher, R. Lico, T. Reiprich, E. Prandini, A. Witzel, Gopal-Krishna, and P. Biermann for very insightful discussions. A. Roy's comments helped to significantly improve this paper. The comments of both anonymous referees provided very constructive help. Michal Zajaček acknowledges the support by the National Science Centre, Poland, grant No. 2017/26/A/ST9/00756 (Maestro 9). This research has made use of data from the MOJAVE database that is maintained by the MOJAVE team [90]. This research has made use of data from the OVRO 40-m monitoring program [89] which is supported in part by NASA grants NNX08AW31G, NNX11A043G, and NNX14AQ89G and NSF grants AST-0808050 and AST-1109911. This research has made use of data from the University of Michigan Radio Astronomy Observatory which has been supported by the University of Michigan and by a series of grants from the National Science Foundation, most recently AST-0607523 and by NASA Fermi GI grants. This work has made use of public Fermi data obtained from the High Energy Astrophysics Science Archive Research Center (HEASARC), provided by NASA Goddard Space Flight Center.

Conflicts of Interest: The authors declare no conflict of interest. 


\section{Appendix A. Difmap Model-Fitting Parameters}
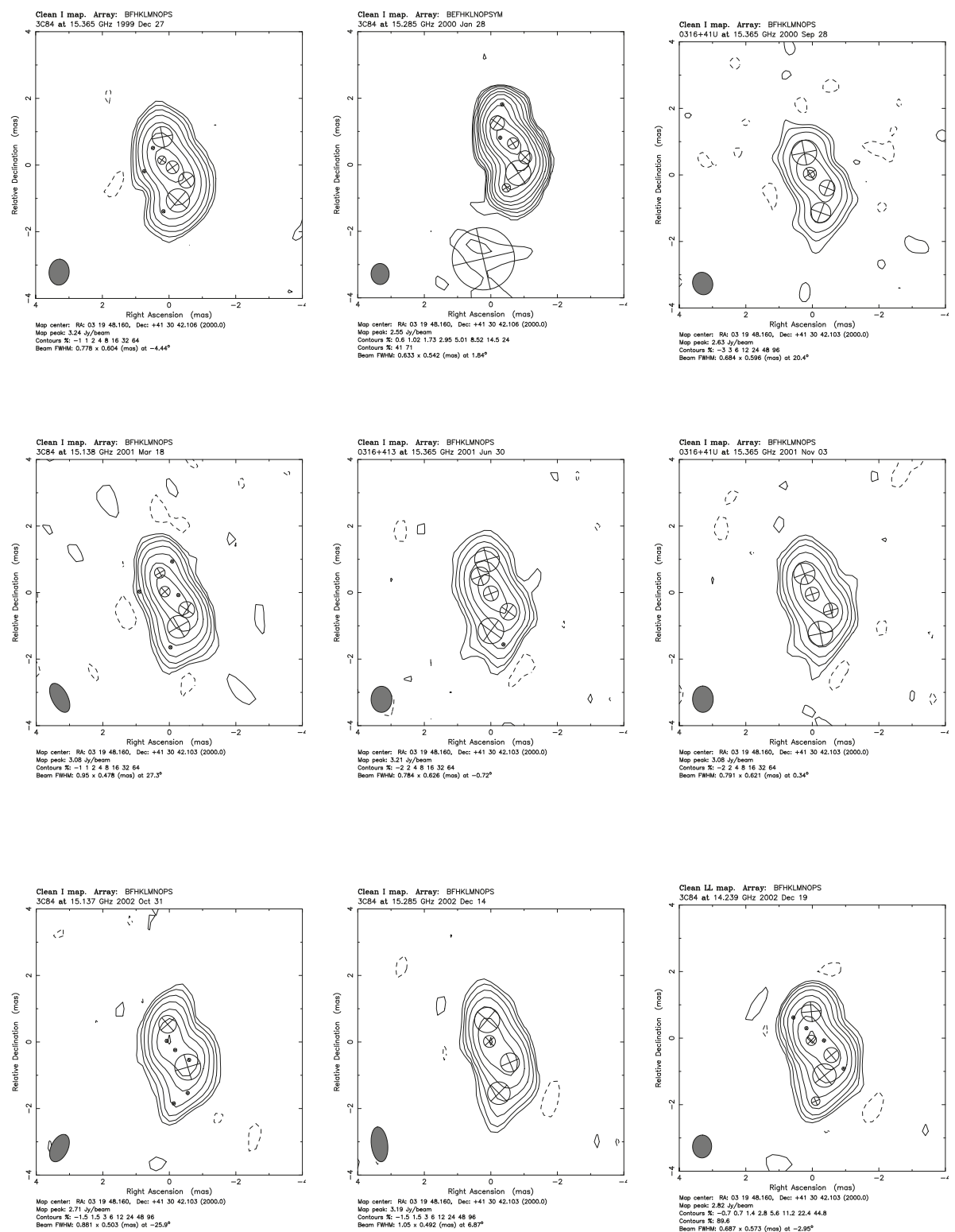

Figure A1. VLBA images superimposed by Gaussian circular components. Their parameters have been derived within the difmap-modelfit programme. The epochs are: December 1999-February 2002. 


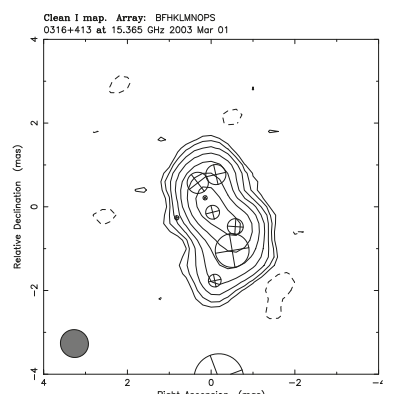

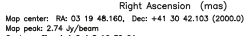

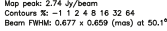

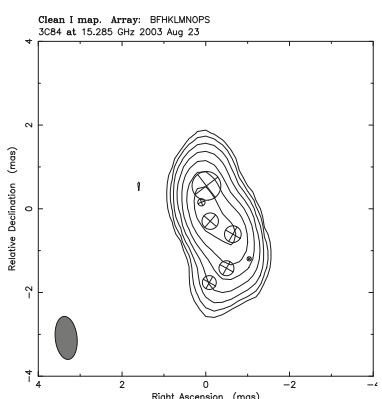

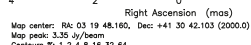

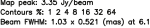

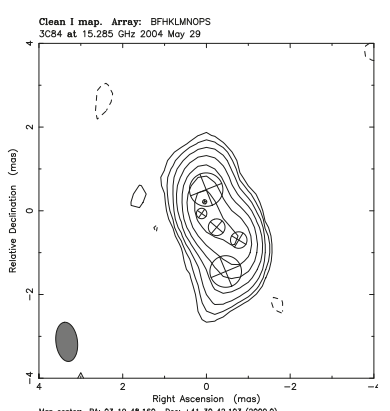

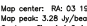

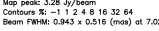

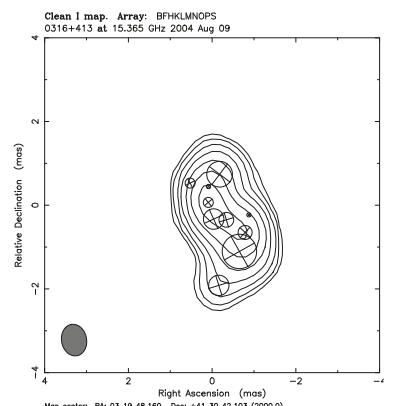

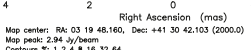

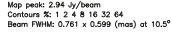

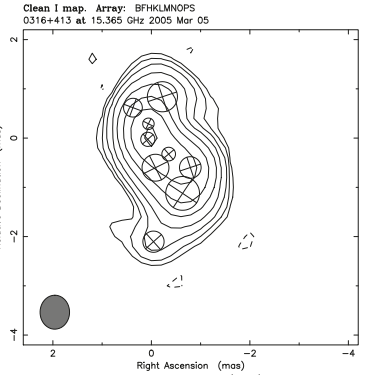

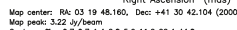

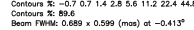

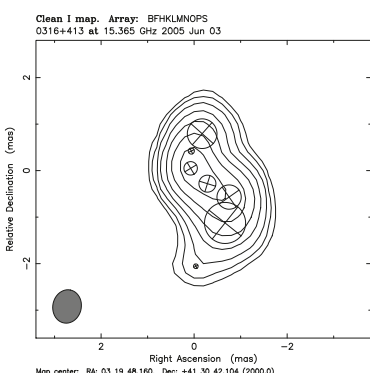

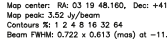

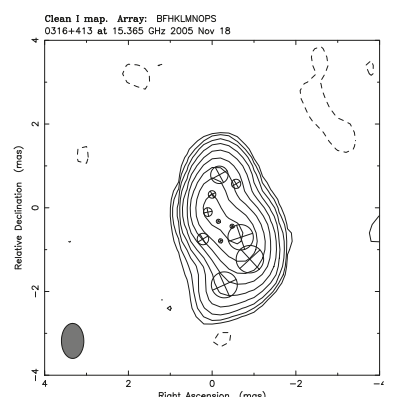

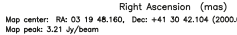

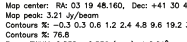

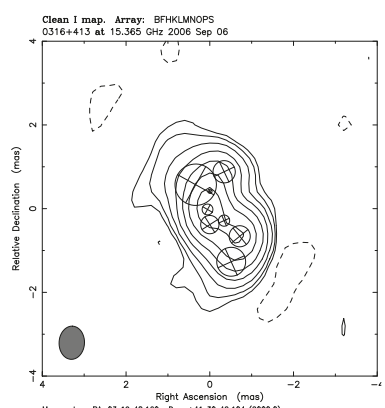

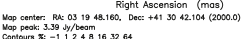

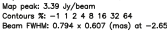

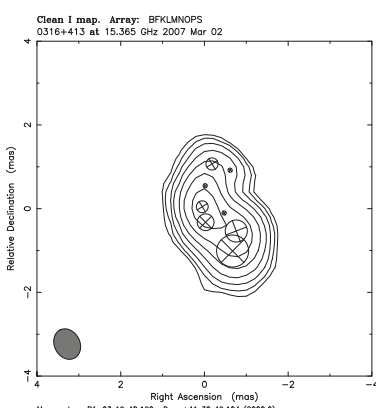

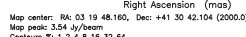

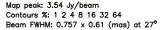

Figure A2. Same as Figure A1 for epochs: March 2003-March 2007. 


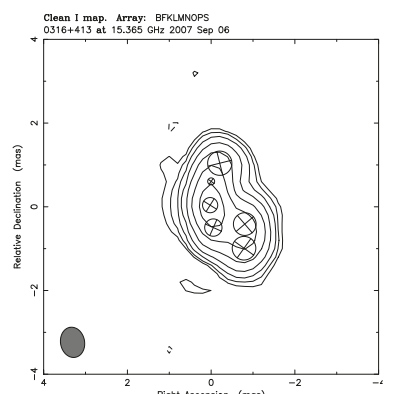

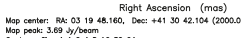

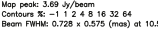

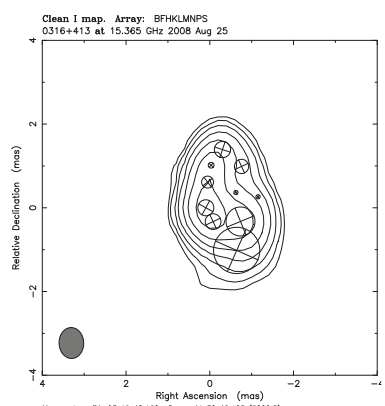

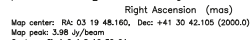

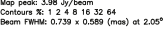

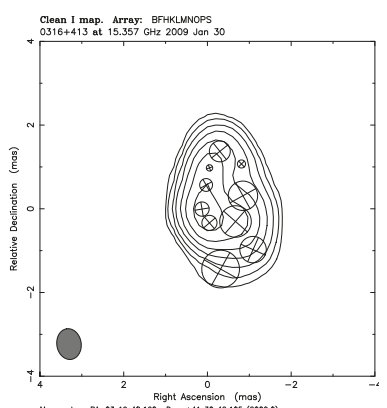

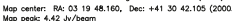

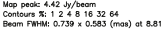

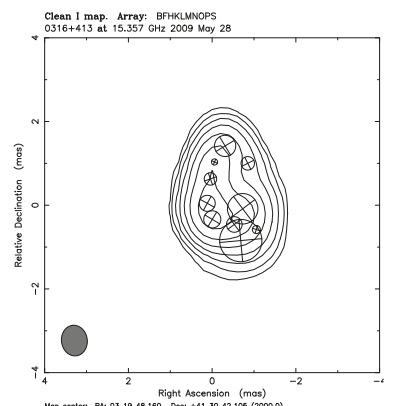

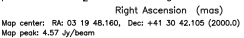

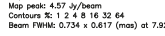

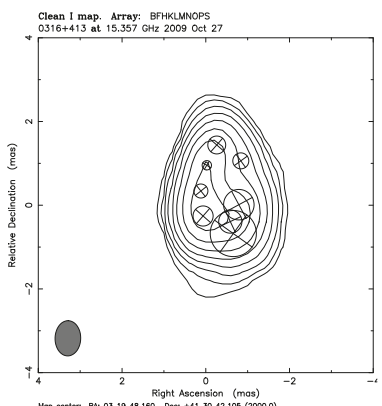

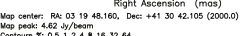

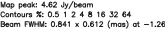

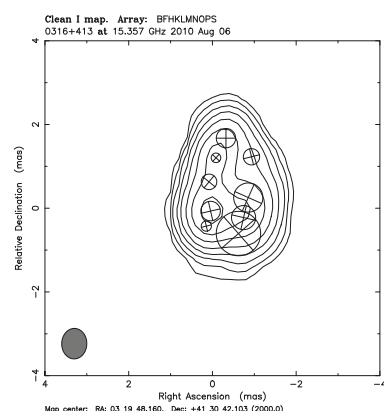

ut

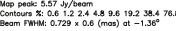

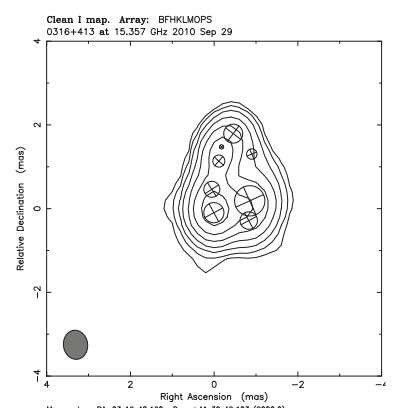

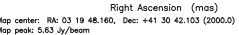

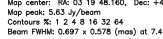

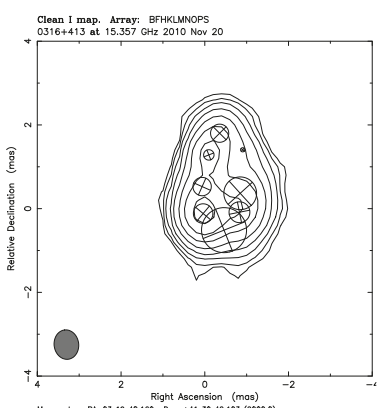

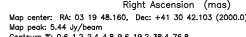

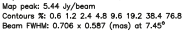

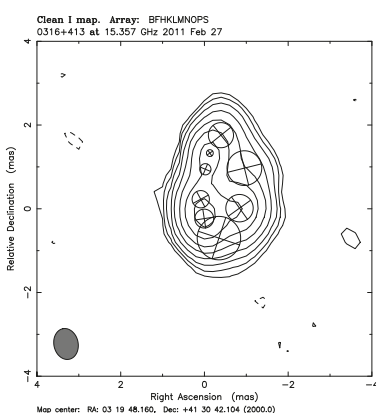

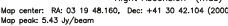
Contraus $x$. 76.8

Figure A3. Same as Figures A1 and A2 for epochs: September 2007-February 2011. 


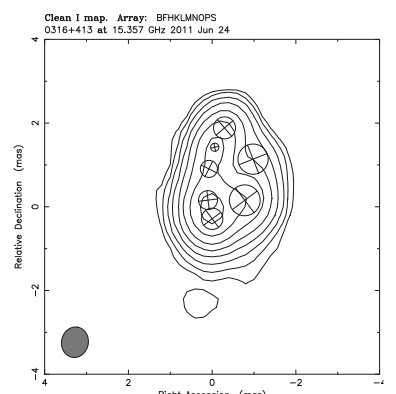

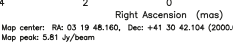

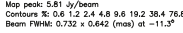

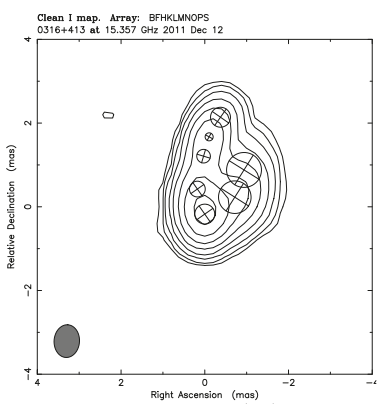

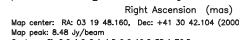

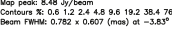

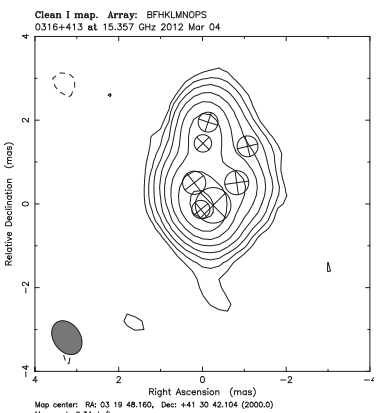

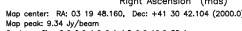

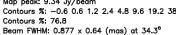

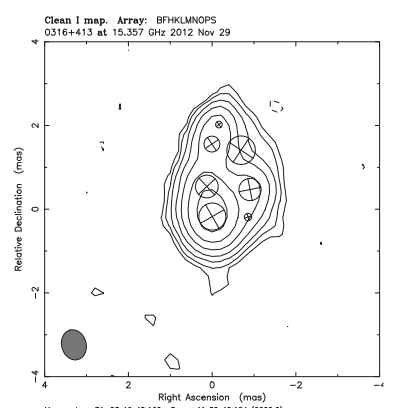

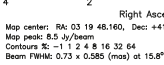

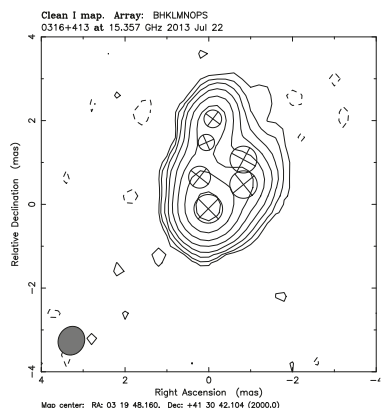

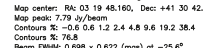
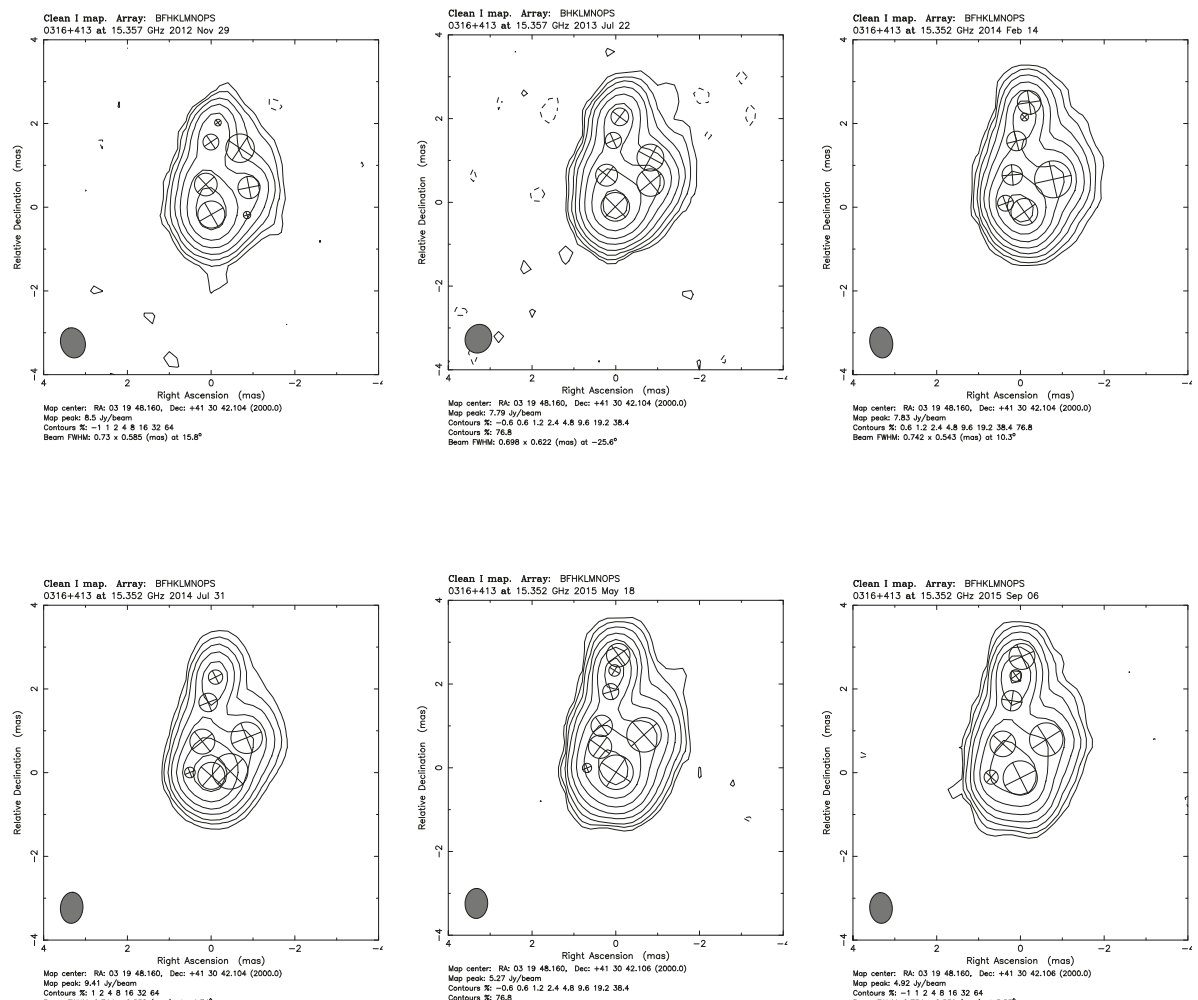

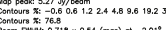

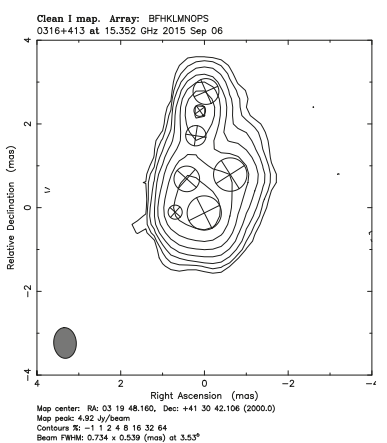

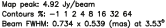

Figure A4. Same as Figures A1-A3 for epochs: June 2011—September 2015. 

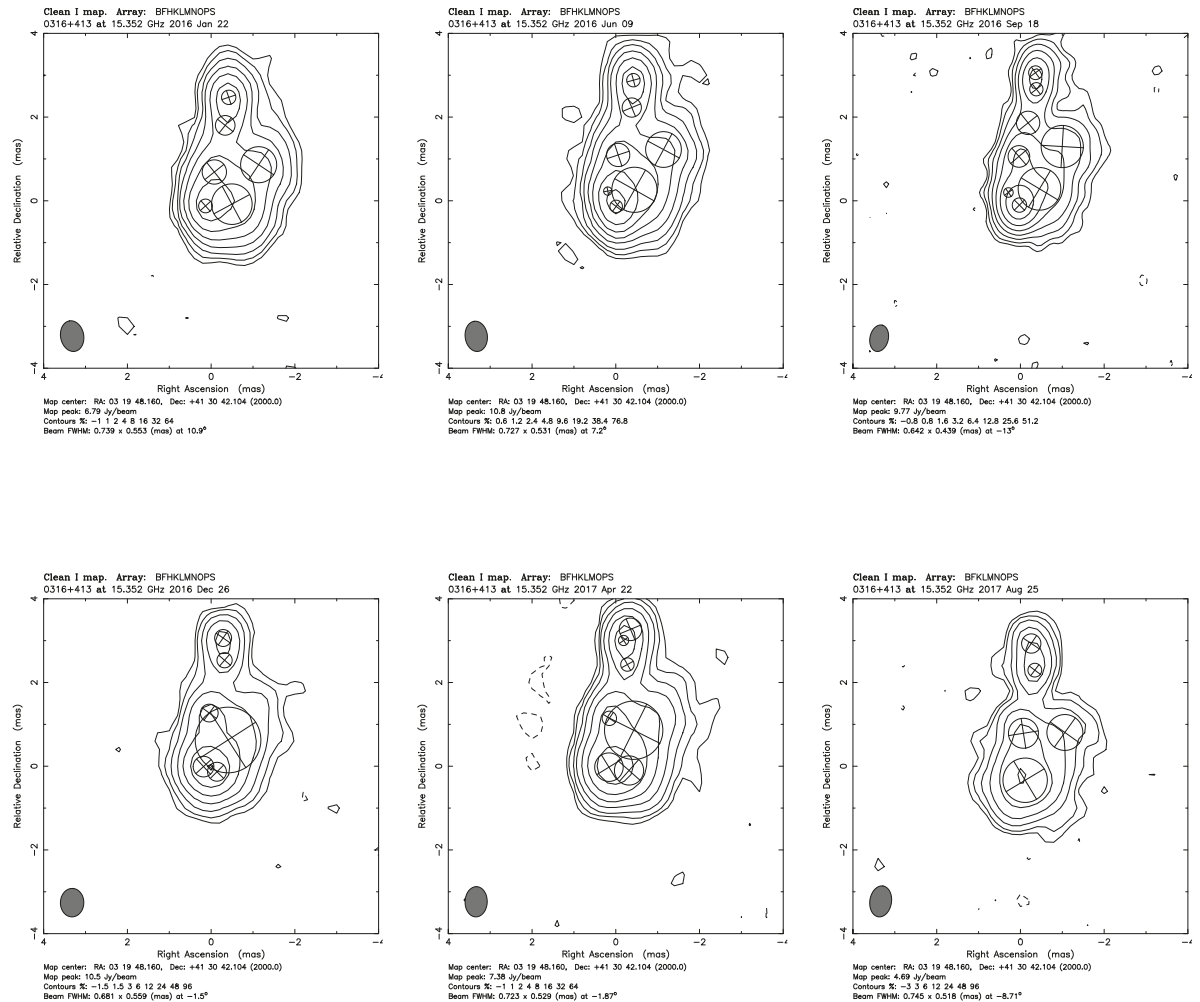

Figure A5. Same as Figures A1-A4 for epochs: January 2016-August 2017.

\section{References and Notes}

1. Nesterov, N.S.; Lyuty, V.M.; Valtaoja, E. Radio and optical evolution of the Seyfert galaxy NGC 1275. Astron. Astrophys. 1995, 296, 628-632.

2. Bridges, T.J.; Irwin, J.A. Molecular gas in the Perseus cooling flow galaxy, NGC 1275. Mon. Not. R. Astron. Soc. 1998, 300, 967-976. [CrossRef]

3. Punsly, B.; Marziani, P.; Bennert, V.N.; Nagai, H.; Gurwell, M.A. Revealing the Broad Line Region of NGC 1275: The Relationship to Jet Power. Astrophys. J. 2018, 869, 143. [CrossRef]

4. Kino, M.; Wajima, K.; Kawakatu, N.; Nagai, H.; Orienti, M.; Giovannini, G.; Hada, K.; Niinuma, K.; Giroletti, M. Evidence of Jet-Clump Interaction: A Flip of the Radio Jet Head of 3C 84. Astrophys. J. 2018, 864, 118. [CrossRef]

5. Hiura, K.; Nagai, H.; Kino, M.; Niinuma, K.; Sorai, K.; Chida, H.; Akiyama, K.; D’Ammando, F.; Giovannini, G.; Giroletti, M.; et al. VERA monitoring of the radio jet 3C 84 in the period of 2007-2013: Detection of non-linear motion. Publ. Astron. Soc. Jpn. 2018, 70, 83. [CrossRef]

6. Hodgson, J.A.; Rani, B.; Lee, S.S.; Algaba, J.C.; Kino, M.; Trippe, S.; Park, J.H.; Zhao, G.Y.; Byun, D.Y.; Kang, S.; et al. KVN observations reveal multiple $\gamma$-ray emission regions in 3C 84? Mon. Not. R. Astron. Soc. 2018, 475, 368-378. [CrossRef]

7. Jorstad, S.G.; Marscher, A.P.; Morozova, D.A.; Troitsky, I.S.; Agudo, I.; Casadio, C.; Foord, A.; Gómez, J.L.; MacDonald, N.R.; Molina, S.N.; et al. Kinematics of Parsec-scale Jets of Gamma-Ray Blazars at 43 GHz within the VLBA-BU-BLAZAR Program. Astrophys. J. 2017, 846, 98. [CrossRef] 
8. Lister, M.L.; Aller, M.F.; Aller, H.D.; Homan, D.C.; Kellermann, K.I.; Kovalev, Y.Y.; Pushkarev, A.B.; Richards, J.L.; Ros, E.; Savolainen, T. MOJAVE: XIII. Parsec-scale AGN Jet Kinematics Analysis Based on 19 years of VLBA Observations at $15 \mathrm{GHz}$. Astron. J. 2016, 152, 12. [CrossRef]

9. Nagai, H.; Haga, T.; Giovannini, G.; Doi, A.; Orienti, M.; D’Ammando, F.; Kino, M.; Nakamura, M.; Asada, K.; Hada, K.; et al. Limb-brightened Jet of 3C 84 Revealed by the $43 \mathrm{GHz}$ Very-Long-Baseline-Array Observation. Astrophys. J. 2014, 785, 53. [CrossRef]

10. Pearson, T.J.; Readhead, A.C.S. The milli-arcsecond structure of a complete sample of radio sources. I. VLBI maps of seven sources. Astrophys. J. 1981, 248, 61-81. [CrossRef]

11. Pauliny-Toth, I.I.K.; Preuss, E.; Witzel, A.; Graham, D.; Kellerman, K.I.; Ronnang, B. 6-cm VLBI observations of compact radio sources. Astron. J. 1981, 86, 371-385. [CrossRef]

12. Walker, R.C.; Anantharamaiah, K.R. A VLBA Search for a Stimulated Recombination Line from the Accretion Region in NGC 1275. Astron. J. 2003, 125, 1756-1761. [CrossRef]

13. Giovannini, G.; Savolainen, T.; Orienti, M.; Nakamura, M.; Nagai, H.; Kino, M.; Giroletti, M.; Hada, K.; Bruni, G.; Kovalev, Y.Y.; et al. A wide and collimated radio jet in 3C84 on the scale of a few hundred gravitational radii. Nat. Astron. 2018, 2, 472-477. [CrossRef]

14. Nagai, H.; Suzuki, K.; Asada, K.; Kino, M.; Kameno, S.; Doi, A.; Inoue, M.; Kataoka, J.; Bach, U.; Hirota, T.; et al. VLBI Monitoring of 3C 84 (NGC 1275) in Early Phase of the 2005 Outburst. Publ. Astron. Soc. Japan 2010, 62, L11. [CrossRef]

15. Suzuki, K.; Nagai, H.; Kino, M.; Kataoka, J.; Asada, K.; Doi, A.; Inoue, M.; Orienti, M.; Giovannini, G.; Giroletti, M.; et al. Exploring the Central Sub-parsec Region of the $\gamma$-Ray Bright Radio Galaxy 3C 84 with VLBA at $43 \mathrm{GHz}$ in the Period of 2002-2008. Astrophys. J. 2012, 746, 140. [CrossRef]

16. Madejski, G.G.; Sikora, M. Gamma-Ray Observations of Active Galactic Nuclei. Annu. Rev. Astron. Astrophys. 2016, 54, 725-760. [CrossRef]

17. Rani, B. Radio Galaxies-The TeV Challenge. Galaxies 2019, 7, 23. [CrossRef]

18. Rieger, F.; Levinson, A. Radio Galaxies at VHE Energies. Galaxies 2018, 6, 116. [CrossRef]

19. Kadowaki, L.H.S.; de Gouveia Dal Pino, E.M.; Singh, C.B. The Role of Fast Magnetic Reconnection on the Radio and Gamma-ray Emission from the Nuclear Regions of Microquasars and Low Luminosity AGNs. Astrophys. J. 2015, 802, 113. [CrossRef]

20. Britzen, S.; Fendt, C.; Eckart, A.; Karas, V. A new view on the M 87 jet origin: Turbulent loading leading to large-scale episodic wiggling. Astron. Astrophys. 2017, 601, A52. [CrossRef]

21. Acciari, V.A.; Aliu, E.; Arlen, T.; Bautista, M.; Beilicke, M.; Benbow, W.; Bradbury, S.M.; Buckley, J.H.; Bugaev, V.; Butt, Y.; et al. Radio Imaging of the Very-High-Energy $\gamma$-Ray Emission Region in the Central Engine of a Radio Galaxy. Science 2009, 325, 444. [CrossRef]

22. Abramowski, A.; Acero, F.; Aharonian, F.; Akhperjanian, A.G.; Anton, G.; Balzer, A.; Barnacka, A.; Barres de Almeida, U.; Becherini, Y.; Becker, J.; et al. The 2010 Very High Energy $\gamma$-Ray Flare and 10 Years of Multi-wavelength Observations of M 87. Astrophys. J. 2012, 746, 151. [CrossRef]

23. Hada, K.; Giroletti, M.; Kino, M.; Giovannini, G.; D’Ammando, F.; Cheung, C.C.; Beilicke, M.; Nagai, H.; Doi, A.; Akiyama, K.; et al. A Strong Radio Brightening at the Jet Base of M 87 during the Elevated Very High Energy Gamma-Ray State in 2012. Astrophys. J. 2014, 788, 165. [CrossRef]

24. Kadler, M.; Eisenacher, D.; Ros, E.; Mannheim, K.; Elsässer, D.; Bach, U. The blazar-like radio structure of the TeV source IC 310. Astron. Astrophys. 2012, 538, L1. [CrossRef]

25. HESS Collaboration; Abdalla, H.; Abramowski, A.; Aharonian, F.; Ait Benkhali, F.; Akhperjanian, A.G.; Andersson, T.; Angüner, E.O.; Arrieta, M.; Aubert, P.; et al. H.E.S.S. discovery of very high energy $\gamma$-ray emission from PKS 0625-354. Mon. Not. R. Astron. Soc. 2018, 476, 4187-4198. [CrossRef]

26. Tavecchio, F.; Ghisellini, G. On the spine-layer scenario for the very high-energy emission of NGC 1275. Mon. Not. R. Astron. Soc. 2014, 443, 1224-1230. [CrossRef]

27. De Angelis, A.; Mallamaci, M. Gamma-ray astrophysics. Eur. Phys. J. Plus 2018, 133, 324. [CrossRef]

28. Sikora, M.; Madejski, G. Blazars; American Institute of Physics Conference Series; Aharonian, F.A., Völk, H.J., Eds.; American Institute: New York, NY, USA, 2001; Volume 558, pp. 275-288. [CrossRef]

29. Aleksić, J.; Alvarez, E.A.; Antonelli, L.A.; Antoranz, P.; Asensio, M.; Backes, M.; Barres de Almeida, U.; Barrio, J.A.; Bastieri, D.; Becerra González, J.; et al. Detection of very-high energy $\gamma$-ray emission from $<$ ASTROBJ>NGC 1275</ASTROBJ> by the MAGIC telescopes. Astron. Astrophys. 2012, 539, L2. [CrossRef] 
30. MAGIC Collaboration; Ansoldi, S.; Antonelli, L.A.; Arcaro, C.; Baack, D.; Babić, A.; Banerjee, B.; Bangale, P.; Barres de Almeida, U.; Barrio, J.A.; et al. Gamma-ray flaring activity of NGC1275 in 2016-2017 measured by MAGIC. Astron. Astrophys. 2018, 617, A91. [CrossRef]

31. Strauss, M.A.; Huchra, J.P.; Davis, M.; Yahil, A.; Fisher, K.B.; Tonry, J. A Redshift Survey of IRAS Galaxies. VII. The Infrared and Redshift Data for the 1.936 Jansky Sample. Astrophys. J. Suppl. 1992, 83, 29. [CrossRef]

32. Ade, P.A.; Aghanim, N.; Arnaud, M.; Ashdown, M.; Aumont, J.; Baccigalupi, C.; Banday, A.J.; Barreiro, R.B.; Bartlett, J.G., Bartolo, N.; et al. Planck 2015 results-xiii. cosmological parameters. Astron. Astrophys. 2016, 594, A13.

33. Shepherd, M.C. Difmap: An Interactive Program for Synthesis Imaging. Bull. Am. Astron. Soc. 1997, 125, 987-989.

34. Efron, B. Bootstrap methods: Another look at the jackknife. Ann. Stat. 1979, 7, 1-26. [CrossRef]

35. Pashchenko, I.N. Using the bootstrap to assess uncertainties of VLBI results-I. The method and image-based errors. Mon. Not. R. Astron. Soc. 2019, 482, 1955-1965. [CrossRef]

36. Caproni, A.; Abraham, Z. Can long-term periodic variability and jet helicity in 3C 120 be explained by jet precession? Mon. Not. R. Astron. Soc. 2004, 349, 1218-1226 [CrossRef]

37. Britzen, S.; Fendt, C.; Witzel, G.; Qian, S.J.; Pashchenko, I.N.; Kurtanidze, O.; Zajacek, M.; Martinez, G.; Karas, V.; Aller, M.; et al. OJ287: Deciphering the 'Rosetta stone of blazars. Mon. Not. R. Astron. Soc. 2018, 478, 3199-3219. [CrossRef]

38. Abraham, Z. Precession, beaming and the periodic light curve of OJ287. Astron. Astrophys. 2000, 355, 915-921.

39. Abraham, Z. Beaming in Jets with Evolving Relativistic Features. Blazar Demogr. Phys. 2001, 227, 108.

40. McCrea, W.H. Relativistic beaming. Mon. Not. R. Astron. Soc. 1972, 157, 359. [CrossRef]

41. Blandford, R.D.; Königl, A. Relativistic jets as compact radio sources. Astrophys. J. 1979, 232, 34-48. [CrossRef]

42. Fabian, A.C.; Sanders, J.S.; Allen, S.W.; Canning, R.E.A.; Churazov, E.; Crawford, C.S.; Forman, W.; Gabany, J.; Hlavacek-Larrondo, J.; Johnstone, R.M.; et al. A wide Chandra view of the core of the Perseus cluster. Mon. Not. R. Astron. Soc. 2011, 418, 2154-2164. [CrossRef]

43. Dunn, R.J.H.; Fabian, A.C.; Sanders, J.S. Precession of the super-massive black hole in NGC 1275 (3C 84)? Mon. Not. R. Astron. Soc. 2006, 366, 758-766. [CrossRef]

44. Levenberg, K. A method for the solution of certain problems in least squares. Quat. J. Appl. Math. 1944, 27 164-168. [CrossRef]

45. Marquardt, D.W. An Algorithm for Least-Squares Estimation of Nonlinear Parameters. J. Soc. Ind. Appl. Math. 1963, 11, 431-441. [CrossRef]

46. Lister, M.L.; Cohen, M.H.; Homan, D.C.; Kadler, M.; Kellermann, K.I.; Kovalev, Y.Y.; Ros, E.; Savolainen, T.; Zensus, J.A. MOJAVE: Monitoring of Jets in Active Galactic Nuclei with VLBA Experiments. VI. Kinematics Analysis of a Complete Sample of Blazar Jets. Astron. J. 2009, 138, 1874-1892. [CrossRef]

47. Britzen, S.; Fendt, C.; Böttcher, M.; et al. Astron. Astrophys. 2019, submitted.

48. Edelson, R.A.; Krolik, J.H. The Discrete Correlation Function: A New Method for Analyzing Unevenly Sampled Variability Data. Astrophys. J. 1988, 333, 646, doi:10.1086/166773. [CrossRef]

49. Emmanoulopoulos, D.; McHardy, I.M.; Papadakis, I.E. Generating artificial light curves: revisited and updated. Mon. Not. R. Astron. Soc. 2013, 433, 907-927. [CrossRef]

50. Rasmussen, C.E.; Williams, C.K.I. Gaussian Processes for Machine Learning; MIT Press: Cambridge, MA, USA, 2006

51. Foreman-Mackey, D.; Hogg, D. W.; Lang, D.; Goodman, J.; emcee: The MCMC Hammer. Publ. Astron. Soc. Pac. 2013. [CrossRef]

52. Ambikasaran, S.; Foreman-Mackey, D.; Greengard, L.; Hogg, D. W.; O’Neil, M. Fast Direct Methods for Gaussian Processes. IEEE Trans. Pattern Anal. Mach. Intell. 2015. [CrossRef]

53. Mattox, J.R.; Bertsch, D.L.; Chiang, J.; Dingus, B.L.; Digel, S.W.; Esposito, J.A.; Fierro, J.M.; Hartman, R.C.; Hunter, S.D.; Kanbach, G.; et al. The Likelihood Analysis of EGRET Data. Astrophys. J. 1996, 461, 396. [CrossRef]

54. Falceta-Gonçalves, D.; Caproni, A.; Abraham, Z.; Teixeira, D.M.; de Gouveia Dal Pino, E.M. Precessing Jets and X-ray Bubbles from NGC 1275 (3C 84) in the Perseus Galaxy Cluster: A View from Three-dimensional Numerical Simulations. Astrophys. J. Lett. 2010, 713, L74-L78. [CrossRef] 
55. Tanada, K.; Kataoka, J.; Arimoto, M.; Akita, M.; Cheung, C.C.; Digel, S.W.; Fukazawa, Y. The Origins of the Gamma-Ray Flux Variations of NGC 1275 Based on Eight Years of Fermi-LAT Observations. Astrophys. J. 2018, 860, 74. [CrossRef]

56. Asada, K.; Kameno, S.; Shen, Z.Q.; Horiuchi, S.; Gabuzda, D.C.; Inoue, M. The Expanding Radio Lobe of 3C 84 Revealed by VSOP Observations. Publ. Astron. Soc. Japan 2006, 58, 261-270. [CrossRef]

57. Katz, J.I. A Precessing Disk in OJ 287? Astrophys. J. 1997, 478, 527-529. [CrossRef]

58. Pringle, J.E. Self-induced warping of accretion discs: non-linear evolution and application to AGN. Mon. Not. R. Astron. Soc. 1997, 292, 136-147. [CrossRef]

59. Minkowski, R. Optical Investigations of Radio Sources (Introductory Lecture); van de Hulst, H.C., Ed.; Radio astronomy; Cambridge University Press: Cambridge, UK, 1957; Volume 4, p. 107.

60. Kent, S.M.; Sargent, W.L.W. Ionization and excitation mechanisms in the filaments around NGC 1275. Astrophys. J. 1979, 230, 667-680. [CrossRef]

61. Donahue, M.; Trauger, J.; 2003, STScI-2003-14.

62. Liu, F.K.; Chen, X. Evolution of Supermassive Black Hole Binaries and Acceleration of Jet Precession in Galactic Nuclei. Astrophys. J. 2007, 671, 1272-1283. [CrossRef]

63. Dutson, K.L.; Edge, A.C.; Hinton, J.A.; Hogan, M.T.; Gurwell, M.A.; Alston, W.N. A non-thermal study of the brightest cluster galaxy NGC 1275-the Gamma-Radio connection over four decades. Mon. Not. R. Astron. Soc. 2014, 442, 2048-2057. [CrossRef]

64. Marscher, A.P.; Gear, W.K. Models for high-frequency radio outbursts in extragalactic sources, with application to the early 1983 millimeter-to-infrared flare of 3C 273. Astrophys. J. 1985, 298, 114-127. [CrossRef]

65. Fuhrmann, L.; Larsson, S.; Chiang, J.; Angelakis, E.; Zensus, J.A.; Nestoras, I.; Krichbaum, T.Â.P.; Ungerechts, H.; Sievers, A.; Pavlidou, V.; et al. Detection of significant $\mathrm{cm}$ to sub-mm band radio and $\gamma$-ray correlated variability in Fermi bright blazars. Mon. Not. R. Astron. Soc. 2014, 441, 1899-1909. [CrossRef]

66. Ramakrishnan, V.; Hovatta, T.; Nieppola, E.; Tornikoski, M.; Lähteenmäki, A.; Valtaoja, E. Locating the $\gamma$-ray emission site in Fermi/LAT blazars from correlation analysis between $37 \mathrm{GHz}$ radio and $\gamma$-ray light curves. Mon. Not. R. Astron. Soc. 2015, 452, 1280-1294. [CrossRef]

67. Max-Moerbeck, W.; Hovatta, T.; Richards, J.L.; King, O.G.; Pearson, T.J.; Readhead, A.C.S.; Reeves, R.; Shepherd, M.C.; Stevenson, M.A.; Angelakis, E.; et al. Time correlation between the radio and gamma-ray activity in blazars and the production site of the gamma-ray emission. Mon. Not. R. Astron. Soc. 2014, 445, 428-436. [CrossRef]

68. Abdo, A.A.; Ackermann, M.; Ajello, M.; Asano, K.; Baldini, L.; Ballet, J.; Barbiellini, G.; Bastieri, D.; Baughman, B.M.; Bechtol, K.; et al. Fermi Discovery of Gamma-ray Emission from NGC 1275. Astrophys. J. 2009, 699, 31-39. [CrossRef]

69. Fukazawa, Y.; Shiki, K.; Tanaka, Y.; Itoh, R.; Takahashi, H.; Imazato, F.; D'Ammando, F.; Ojha, R.; Nagai, H. X-Ray and GeV Gamma-Ray Variability of the Radio Galaxy NGC 1275. Astrophys. J. 2018, 855, 93. [CrossRef]

70. Aleksić, J.; Ansoldi, S.; Antonelli, L.A.; Antoranz, P.; Babic, A.; Bangale, P.; Barres de Almeida, U.; Barrio, J.A.; Becerra González, J.; Bednarek, W.; et al. Contemporaneous observations of the radio galaxy NGC 1275 from radio to very high energy $\gamma$-rays. Astron. Astrophys. 2014, 564, A5. [CrossRef]

71. Benbow, W.; VERITAS Collaboration. Highlights from the VERITAS AGN Observation Program. In Proceedings of the 34th International Cosmic Ray Conference (ICRC2015), The Hague, The Netherlands, 30 July-6 August 2015; Volume 34, p. 821.

72. Ahnen, M.L.; Ansoldi, S.; Antonelli, L.A.; Antoranz, P.; Babic, A.; Banerjee, B.; Bangale, P.; Barres de Almeida, U.; Barrio, J.A.; Becerra González, J.; et al. Deep observation of the NGC 1275 region with MAGIC: search of diffuse $\gamma$-ray emission from cosmic rays in the Perseus cluster. Astron. Astrophys. 2016, 589, A33. [CrossRef]

73. Acciari, V.A.; Ansoldi, S.; Antonelli, L.A.; Arbet Engels, A.; Arcaro, C.; Baack, D.; Babić, A.; Banerjee, B.; Bangale, P.; Barres de Almeida, U.; et al. Constraining dark matter lifetime with a deep gamma-ray survey of the Perseus galaxy cluster with MAGIC. Phys. Dark Univ. 2018, 22, 38-47. [CrossRef]

74. Sanders, J.S.; Fabian, A.C. Hard X-ray emission from the core of the Perseus cluster and the thermal content of the radio bubbles. In Heating Versus Cooling in Galaxies and Clusters of Galaxies; Böhringer, H., Pratt, G.W., Finoguenov, A., Schuecker, P., Eds.; Springer: Berlin/Heidelberg, Germany, 2007; p. 74. [CrossRef] 
75. Dosopoulou, F.; Antonini, F. Dynamical Friction and the Evolution of Supermassive Black Hole Binaries: The Final Hundred-parsec Problem. Astrophys. J. 2017, 840, 31. [CrossRef]

76. Lu, J.F. $\alpha$-Disks and the precession of extragalactic jets. Acta Astrophys. Sin. 1992, 12, 9. [CrossRef]

77. Blandford, R.D.; Znajek, R.L. Electromagnetic extraction of energy from Kerr black holes. Mon. Not. R. Astron. Soc. 1977, 179, 433-456. [CrossRef]

78. Blandford, R.D.; Payne, D.G. Hydromagnetic flows from accretion disks and the production of radio jets. Mon. Not. R. Astron. Soc. 1982, 199, 883-903. [CrossRef]

79. Naab, T.; Ostriker, J.P. Theoretical Challenges in Galaxy Formation. Annu. Rev. Astron. Astrophys. 2017, 55, 59-109. [CrossRef]

80. Begelman, M.C.; Blandford, R.D.; Rees, M.J. Massive black hole binaries in active galactic nuclei. Nature 1980, 287, 307-309. [CrossRef]

81. Merritt, D.; Milosavljević, M. Massive Black Hole Binary Evolution. Liv. Rev. Relat. 2005, 8, 8. [CrossRef]

82. Komossa, S.; Burwitz, V.; Hasinger, G.; Predehl, P.; Kaastra, J.S.; Ikebe, Y. Discovery of a Binary Active Galactic Nucleus in the Ultraluminous Infrared Galaxy NGC 6240 Using Chandra. Astrophys. J. Lett. 2003, 582, L15-L19. [CrossRef]

83. Kun, E.; Gabányi, K.É.; Karouzos, M.; Britzen, S.; Gergely, L.Á. A spinning supermassive black hole binary model consistent with VLBI observations of the S5 $1928+738$ jet. Mon. Not. R. Astron. Soc. 2014, 445, 1370-1382. [CrossRef]

84. Qian, S.J.; Kudryavtseva, N.A.; Britzen, S.; Krichbaum, T.P.; Gao, L.; Witzel, A.; Zensus, J.A.; Aller, M.F.; Aller, H.D.; Zhang, X.Z. A Possible Periodicity in the Radio Light Curves of 3C 454.3. Chin. J. Astron. Astrophys. 2007, 7, 364-374. [CrossRef]

85. Caproni, A.; Abraham, Z; Motter, J.C.; Monteiro, H. Jet Precession Driven by a Supermassive Black Hole Binary System in the BL Lac Object PG 1553+113. Astrophys. J. Lett. 2017, 851, L39. [CrossRef]

86. Treister, E.; Schawinski, K.; Urry, C.M.; Simmons, B.D. Major Galaxy Mergers Only Trigger the Most Luminous Active Galactic Nuclei. Astrophys. J. Lett. 2012, 758, L39. [CrossRef]

87. Ramos Almeida, C.; Tadhunter, C.N.; Inskip, K.J.; Morganti, R.; Holt, J.; Dicken, D. The optical morphologies of the 2 Jy sample of radio galaxies: evidence for galaxy interactions. Mon. Not. R. Astron. Soc. 2011, 410, 1550-1576. [CrossRef]

88. Chiaberge, M.; Gilli, R.; Lotz, J.M.; Norman, C. Radio Loud AGNs are Mergers. Astrophys. J. 2015, 806, 147. [CrossRef]

89. Richards, J.L.; Max-Moerbeck, W.; Pavlidou, V.; King, O.G.; Pearson, T.J.; Readhead, A.C.S.; Reeves, R.; Shepherd, M.C.; Stevenson, M.A.; Weintraub, L.C.; et al. Blazars in the Fermi Era: The OVRO 40 m Telescope Monitoring Program. Astrophys. J. Suppl. 2011, 194, 29. [CrossRef]

90. Lister, M.L.; Aller, M.F.; Aller, H.D.; Hodge, M.A.; Homan, D.C.; Kovalev, Y.Y.; Pushkarev, A.B.; Savolainen, T. MOJAVE. XV. VLBA 15 GHz Total Intensity and Polarization Maps of 437 Parsec-scale AGN Jets from 1996 to 2017. Astrophys. J. Suppl. 2018, 234, 12. [CrossRef]

(C) 2019 by the authors. Licensee MDPI, Basel, Switzerland. This article is an open access article distributed under the terms and conditions of the Creative Commons Attribution (CC BY) license (http:/ / creativecommons.org/licenses/by/4.0/). 


\title{
The High Energy View of FR0 Radio Galaxies
}

\author{
Ranieri Diego Baldi ${ }^{1,2, *}$, Eleonora Torresi ${ }^{3}$, Giulia Migliori ${ }^{4,5}$ and Barbara Balmaverde ${ }^{6}$ \\ 1 Department of Physics and Astronomy, University of Southampton, Highfield SO171BJ, UK \\ 2 Department of Physics, University of Turin, via Pietro Giuria 1, I-10125 Torino, Italy \\ 3 INAF-Astrophysics and Space Science Observatory of Bologna, Via Gobetti 101, I-40129 Bologna, Italy; \\ eleonora.torresi@inaf.it \\ 4 Department of Physics and Astronomy, University of Bologna, Via Gobetti 93/2, 40129 Bologna, Italy; \\ giulia.migliori@inaf.it \\ 5 INAF-Institute of Radio Astronomy, Bologna, Via Gobetti 101, I-40129 Bologna, Italy \\ 6 INAF-Observatory of Turin, via Osservatorio 20, 10025 Pino Torinese, Italy; barbara.balmaverde@inaf.it \\ * Correspondence: r.baldi@soton.ac.uk
}

Received: 22 June 2019; Accepted: 2 September 2019; Published: 5 September 2019

\begin{abstract}
A new class of low-power compact radio sources with limited jet structures, named FR 0, is emerging from recent radio-optical surveys. This abundant population of radio galaxies, five times more numerous than FR Is in the local Universe $(z<0.05)$, represent a potentially interesting target at high and very-high energies (greater than $100 \mathrm{GeV}$ ), as demonstrated by a single case of Fermi detection. Furthermore, these radio galaxies have been recently claimed to contribute non-negligibly to the extra-galactic $\gamma$-ray background and to be possible cosmic neutrino emitters. Here, we review the radio through $\mathrm{X}$-ray properties of $\mathrm{FR} 0$ s to predict their high-energy emission (from $\mathrm{MeV}$ to $\mathrm{TeV}$ ), in light of the near-future facilities operating in this band.
\end{abstract}

Keywords: active galactic nuclei; radio galaxies; gamma-rays; jets; emission: non-thermal; $\mathrm{GeV}$ $\gamma$-rays; $\mathrm{TeV} \gamma$-rays

\section{Introduction}

Radio galaxies (RGs) associated with the most massive black holes (BHs) in the Universe, are important laboratories to study the jet launching mechanism and the production of high and very high energy ( $\mathrm{HE}>100 \mathrm{MeV}$; VHE $>100 \mathrm{GeV}$ ) radiation by Compton scattering due to the presence of relativistic particles.

Although compact RGs were already known to be associated with massive early-type galaxies (ETGs) since the early 1970s (e.g., [1-7]), studies of much brighter extended RGs prevailed because of their more interesting and richer jetted structures. These RGs are classified based on their radio morphology, distinguishing between edge-darkened (FR I type) and edge-brightened (FR II type) sources [8] (Figure 1). Nevertheless, in the last decade, a renewed interest towards low-luminosity RGs (e.g., [9]) is driven by the advent of large-area high-sensitivity optical and radio surveys: the cross-correlation of the Sloan Digital Sky Survey (SDSS, [10]), the Faint Images of the Radio Sky at Twenty centimetres survey (FIRST, [11]) and the National Radio Astronomy Observatory Very Large Array (VLA) Sky Survey (NVSS, [12]) allowed unbiased statistical studies of the radio-AGN activity at low luminosities. With this technique, the authors in $[13,14]$ selected a sample of low-power $\sim 18,000$ RGs up to $\mathrm{z} \sim 0.3$ with radio fluxes higher than $5 \mathrm{mJy}$ at $1.4 \mathrm{GHz}$, covering the range of radio luminosity $L_{1.4 \mathrm{GHz}} \sim 10^{22}-10^{26} \mathrm{~W} \mathrm{~Hz}^{-1}$. All radio morphologies are represented, including twin-jets and core-jet FR Is, narrow and wide angle tails, and FR IIs. However, most of them $(\sim 80 \%)$ appear unresolved or barely resolved at the $5^{\prime \prime}$ FIRST resolution, corresponding to a size limit of $\sim 10 \mathrm{kpc}$. This enormous population of low-luminosity compact RGs, different from FR I/IIs, are still virtually unexplored. Recently, the authors in $[15,16]$ concluded (confirmed later by other independent radio 
studies [17-19]) that this new population of RGs, characterized by a paucity of extended radio emission, dominates over FR Is and FR IIs in the local Universe. For their radio morphological peculiarity, this class of sources has been named as FR 0 in contrast to the classical Fanaroff-Riley classes [20-22]. This new class represents a radical change in our view of the radio sky because our past knowledge about RGs was biased towards powerful extended (from hundreds of $\mathrm{kpc}$ to $\mathrm{Mpc}$ ) radio sources, as selected by low-frequency high-flux surveys (see e.g., the Third Cambridge (3C) catalogue ${ }^{1}$ [23]). However, up to now, the paucity of detailed multi-band information on FR 0s limits our comprehension on the origin of this class and the cause of their confined jet structures.
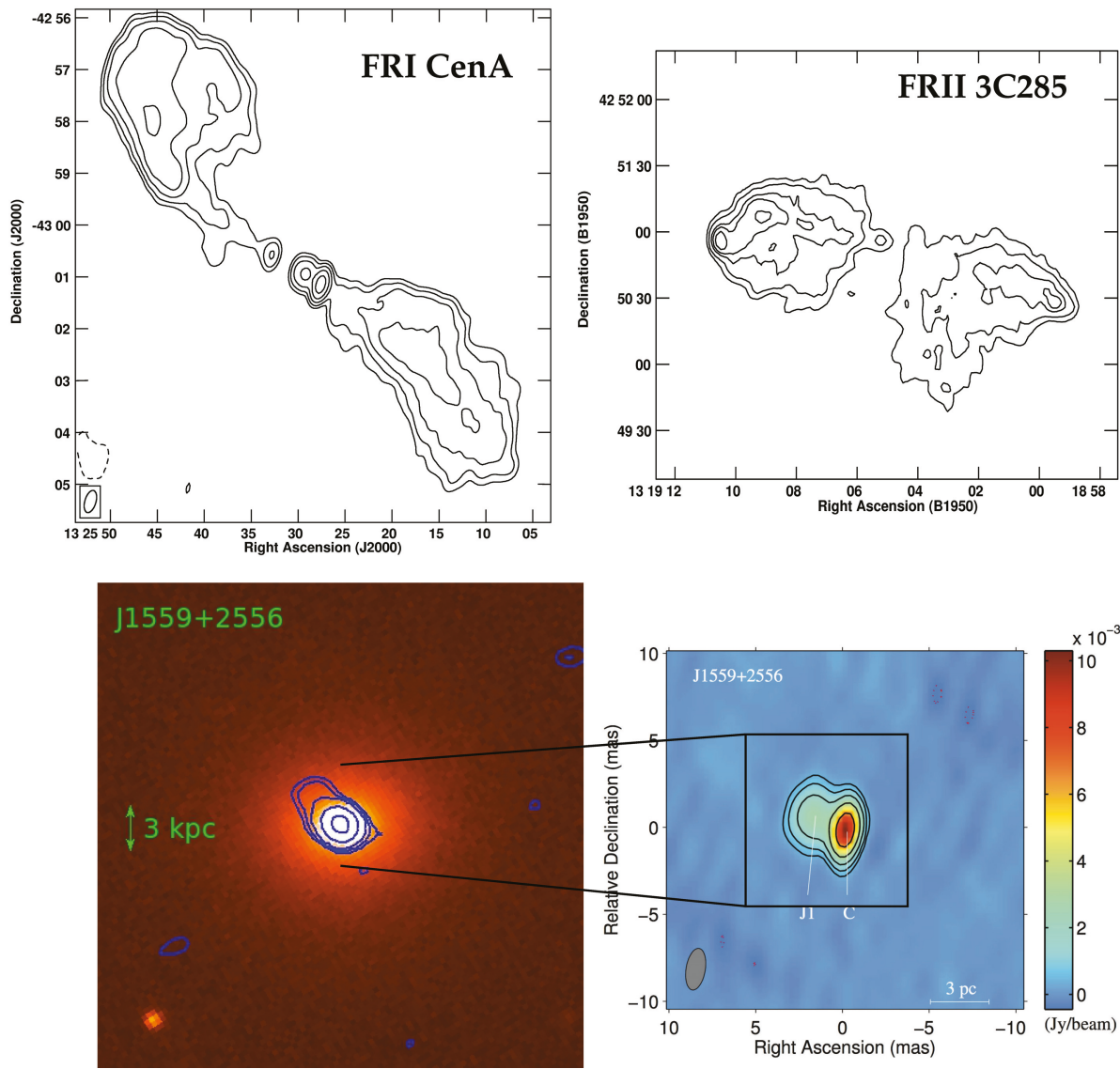

Figure 1. Multi-band composite panel of radio galaxies. On the top are two examples of radio morphologies of a core-brightened FR I (Centaurus A [24] at 1.4 GHz) and an edge-brightened FR II (3C 285, [25] at $1.4 \mathrm{GHz}$ ). On the bottom, we show an example of FR 0, J1559 + 2556. The left panel displays the r-band SDSS image of the elliptical galaxy which hosts the FR 0 with the blue $4.5-\mathrm{GHz}$ radio contours from $V L A$ taken from [26] (3 kpc scale set by the green arrow). The right panel represents the high-resolution zoom on the radio core (on the scale of 3 pc) provided by the VLBI image from [27].

1 The 3C catalogue selects radio sources with flux densities higher than $9 \mathrm{Jy}$ at $178 \mathrm{MHz}$. 
In this review, we summarize the state-of-the-art of multi-wavelength studies of FR 0s, lingering on their emission at high energies from $\mathrm{keV}$ to $\mathrm{TeV}$. In particular, we will focus on the FR 0 population as possible $\gamma$-ray emitters based on one case of HE detection (Tol 1326-379, [28]), current models and facilities, and how their limited jets could accelerate particles that up-scatter low-energy photons or collide to eventually produce HE electromagnetic cascades.

\section{Broadband Properties: From Radio to X-rays}

These compact radio sources have been carefully selected and later included in a catalogue, named FR0CAT [29]. The selected 104 FR 0 s with $\mathrm{z} \leq 0.05$, live in red massive $\left(\gtrsim 10^{11} \mathrm{M}_{\odot}\right)$ ETGs with large $\mathrm{BH}$ masses $\left(\sim 10^{7.5}-10^{9} \mathrm{M}_{\odot}\right)$, and are spectroscopically classified as Low Excitation Galaxies (LEG) [16]: these host properties are similar to those seen for the hosts of 3C/FR Is, but they are on average a factor $\sim 1.6$ less massive $[19,29]$. The radio, optical line, $X$-ray luminosities of FR 0 s generally match those of the 3C/FR Is $[21,26,30]$. The only feature distinguishing the two classes is the paucity of extended radio emission of FR 0s, which turns in a core dominance ${ }^{2}$ higher than classical FR Is by a factor $\sim 30$. They show a strong deficit of total radio emission with respect to the $3 \mathrm{C}$ sources, being 100 times fainter at the same level of (O III) line luminosity, a proxy of the bolometric AGN power [16] (Figure 2). A similar high core dominance has been also observed in nearby giant ETGs that harbour low-power radio-loud AGN (10 ${ }^{36-38} \mathrm{erg} \mathrm{s}^{-1}$ [15,22]), named Core Galaxies (CoreG [31]). These CoreG are basically miniature RGs with nuclei that are the scaled-down version of those of FR Is in terms of AGN bolometric luminosity, jet power and accretion rates $[32,33]$

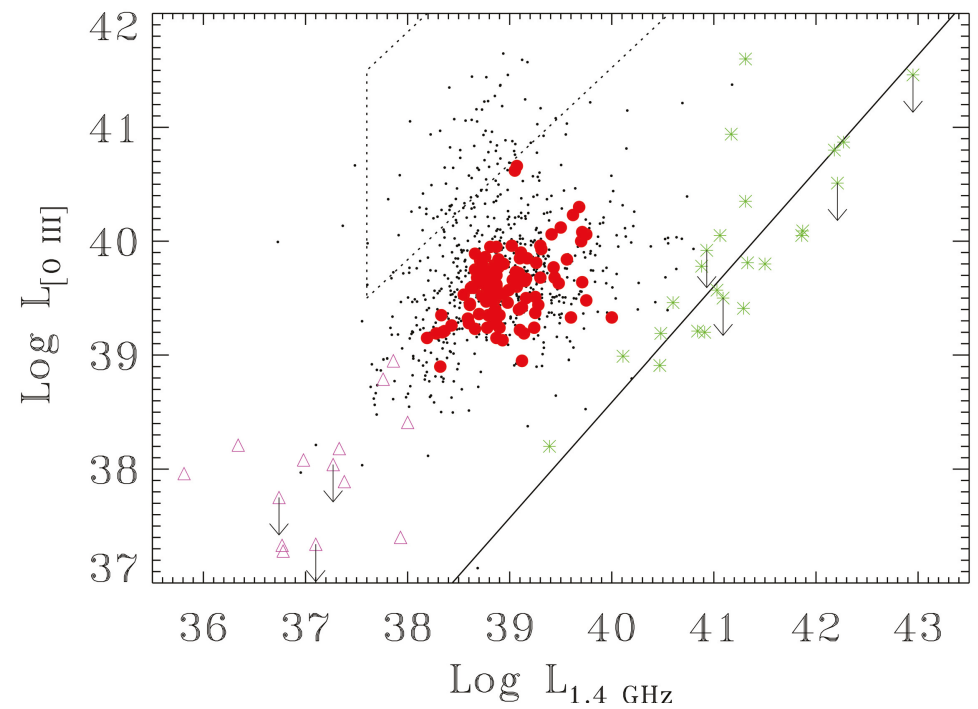

Figure 2. FIRST 1.4-GHz radio vs. [O III] line luminosity $\left(\mathrm{erg} \mathrm{s}^{-1}\right)$. The small points correspond to the low-luminosity RG sample selected by [14]. The solid line represents the correlation between line and radio-luminosity derived for the 3CR/FR I sample (green stars). The dotted lines include the region where radio-quiet Seyfert galaxies are found. The red points are the FR 0 included in the FR0CAT [29] and the violet triangles are the CoreG.

However, the available multi-band data for FR 0s to study their nuclear activity are extremely limited. In the radio band, generally only $1.4 \mathrm{GHz}$ FIRST and NVSS images with shallow angular

2 The core dominance is the ratio between the emission from the unresolved radio core and the total radio emission of the RG. 
resolution ( $5^{\prime \prime}$ and $45^{\prime \prime}$, respectively) were available. Hence, in the last five years, several campaigns with the VLA have been performed to zoom in the GHz-band emission at higher resolution, reaching $0.2^{\prime \prime}[21,26]$. At the kpc scale, the VLA analysis reveals that FR 0s remain compact below $\lesssim 1 \mathrm{kpc}$, with only $\sim 25 \%$ of the sample able to emanate twin or one-sided jets extended for at most a few kpc (Figure 1 as an example). At the parsec scale, higher-resolution radio observations with the Very Large Baseline Interferometer (VLBI) [27] show resolved radio jets for $80 \%$ of the sample. The VLBI multi-epoch data and the symmetry of the radio structures indicate that the jet bulk speed is mildly relativistic (between $0.23 c$ and $0.49 c$ ) with Doppler-boosting factors ranging from 1.7 to 6 .

Including low-frequency radio data-points from the Low-Frequency Array (LOFAR) and the Giant Metrewave Radio Telescope (GMRT) surveys, the FR 0 radio spectra from hundreds of $\mathrm{MHz}$ to several GHz are typically flat $([21,26,34])$. However, their SEDs can be generally described as a gentle spectral curvature towards higher radio frequencies, broader than what is seen in young radio sources. The flat-spectrum radio core powers tightly correlate with the AGN bolometric luminosities, following the relation of FR Is and CoreG [21,26]: this clearly indicates a common central engine, where the jet is the main contributor to the whole bolometric power. At higher radio frequencies, a survey at $15 \mathrm{GHz}$ has been planned with the Arcminute Microkelvin Imager (AMI) array.

In the optical band, the continuum and spectral information of the nuclei are limited to the SDSS data, which yield to a low luminosity of the central source and a prominent radio loudness ${ }^{3}$.

\subsection{X-ray Properties of FR Os}

At higher energies, the properties of FR 0s are almost unexplored. The authors in [30] performed the first systematic study in the X-ray $(2-10 \mathrm{keV})$ band of a sample of 19 FR 0 galaxies selected from [14] on the basis of: (i) redshift $\mathrm{z} \leq 0.15$, (ii) radio size $\leq 10 \mathrm{kpc}$ (in FIRST images); (iii) FIRST flux >30 mJy; (iv) LEG optical classification; (v) having available X-ray data in the public archives of the XMM-Newton, Chandra and Swift satellites. The aforementioned criteria guaranteed that the considered objects are classified as FR 0s, although at higher radio flux densities than the FR0CAT sources. Finally, Tol 1326-379, the first FR 0 radio galaxy detected in $\gamma$-rays (see Section 4), was added to the sample. The principal results of this study are:

1. The X-ray spectra of FR 0s are generally well represented by a power-law absorbed by a Galactic column density. An additional intrinsic absorber is not required by the data suggesting that, in these sources, the circum-nuclear environment is depleted of cold matter (e.g., the dusty torus is missing). In some cases, the addition of a thermal component is required by the data: this soft $\mathrm{X}$-ray emission could be related to extended intergalactic medium or to the hot corona typical of ETGs [36]. The spectral slope of the power-law is generally steep, $\langle\Gamma\rangle=1.9 \pm 0.3$. Only in two cases, which includes Tol 1326-379, is the photon index flatter, $~ 1.2$;

2. FR 0s span a range in X-ray luminosity $\mathrm{L}_{X}=10^{40}-10^{43} \mathrm{erg} \mathrm{s}^{-1}$, similar to FR Is. When the X-ray luminosity is compared to the radio core one, a clear correlation is attested. This points towards a non-thermal origin (i.e., the jet) of the X-ray emission in FR 0s as commonly believed in FR Is (e.g., $[32,37,38])$ (Figure 3);

3. the central engine of FR Os is probably powered by radiatively-inefficient accretion disc (i.e., Advection Dominated Accretion Flow [ADAF] model, [39]), as suggested by the small values of the Eddington-scaled luminosities, $\dot{L}=\mathrm{L}_{b o l} / \mathrm{L}_{E d d} \sim 10^{-3}-10^{-5}$.

3 Radio loudness, $R$, is defined as ratio between the flux densities in the radio $(6 \mathrm{~cm}, 5 \mathrm{GHz})$ and in the optical band (4400 $\AA$ ) [35]; where radio-loud AGN have $R>10$. Assuming the optical galaxy emission as an upper limit on the optical nuclear component and $5 \mathrm{mJy}$ as minimum radio flux, the radio loudness of FR $0 \mathrm{~s}$ is at least $>11$. 


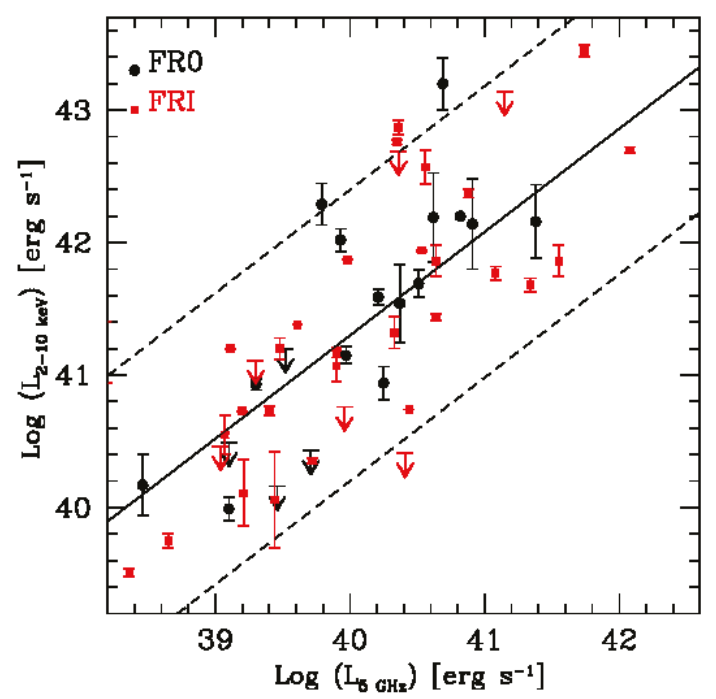

Figure 3. X-ray (2-10 keV) luminosity versus radio core $(5 \mathrm{GHz})$ luminosity (in erg s ${ }^{-1}$ ) for FR 0s (black circles) and FR Is (red squares). Upper limits are represented by arrows. The black solid line is the linear regression for FR 0s + FR Is: $\log L_{X}=(7.8 \pm 0.6)+(0.8 \pm 0.1) \log L_{5} \mathrm{GHz}$. Black dashed lines are uncertainties on the slope, taken from [30].

\subsection{The Uniqueness of the FR 0 Class}

What makes FR 0 class important is their large abundance, being five times more numerous than $3 C / F R$ Is at $z<0.05$. Even at low radio frequencies where optically-thin radio jet emission is expected to dominate over the flat-spectrum core emission, in the LOFAR survey (the Low-Frequency Array Two-Metre Sky Survey LoTSS, [40]), 70\% of RGs appears to be low-luminosity unresolved sources $[41,42]$ at the same angular resolution of FIRST images $\left(5^{\prime \prime}\right)$ : about a few thousand of these compact RGs are potentially FR 0 candidates with respect to a comparable number of extended FR Is and FR IIs [43].

What makes this class of RGs unique is their lack of substantial extended radio emission and consequent high core dominance although they harbour an FR I-like nucleus. Are they low-power blazars? No, the more-preferentially symmetric jet structures of the FR 0s on a kpc scale rule out a significant projection effect [21,26]. The similar radio core-bolometric luminosity correlations, derived from [O III], X-ray, of FR 0s and FR Is yield to their common radio misalignment, since emission line and $\mathrm{HE}$ emission are typically considered independent of orientation. Another proof of the misaligned nature of FR 0s comes from the distribution of the ratio between [O III] and X-ray luminosities $\left(\mathrm{R}_{[O I I I] / X}\right)$. While FR 0 s and FR Is show similar values of $\mathrm{R}_{[O I I I] / X} \sim-1.7$ (e.g., [38,44]), low-luminosity blazars have $\mathrm{R}_{[O I I I] / X} \sim-3.3$ (e.g., [30,45]) as expected if the $\mathrm{X}$-ray emission is beamed. Are they small fading FR Is? A past FR I radio activity is ruled out by the non-detection of extended diffuse emission at low radio frequencies and low angular resolutions (LOFAR and GMRT [34]. The FR 0 nuclear affinity with FR Is is, furthermore, downsized by the the mildly relativistic jet speed measured from VLBI with respect to the still relativistic speeds at parsec scales of FR Is [46-48]. Are they young FR Is? An age scenario, for which FR 0s are young RGs that will all eventually evolve into extended radio sources cannot be generally reconciled with the larger space density of FR 0s than FR Is [26]. Nevertheless, a possible contribution of $\sim 10 \%$ of genuinely young radio sources cannot be excluded among FR 0s (see [17]), based on the fraction of sources with inverted radio spectra [34]. Unlike FR 0s, genuinely young radio sources show different multi-band characteristics: (i) GHz-peaked radio spectra 
due to strong synchrotron self-absorption [49]; (ii) typically brighter in radio and optical line due to the enhanced emission caused by an expanding radio cocoon in a dense gas-rich environment [50]; (iii) FR II-like bolometric and HE powers associated with a radiatively efficient accretion disc (e.g., [51-56]); (iv) often show signatures of intrinsic H I and X-ray absorption [56,57], which suggest that these sources are typically embedded in a rich interstellar medium.

All these characteristics and differences with respect to the other FR classes, blazars, young sources and radio-quiet AGNs point to FR0s as a stand-alone class, which does not fully fit in a orientation-dependent AGN unification scheme [58] a priori, but does in a possible evolutionary AGN scheme [59]. Furthermore, no clear incongruity in the nuclear and host properties of the FR 0s with respect to the other RGs can account for the different radio properties.

A similar class of low-luminosity compact RGs (LLC) have been recently addressed by [60], as kpc-scale radio sources with possibly fading steep-spectrum disrupted jets and short-lived activity because of a lack of significant fuelling onto the BH [61,62]. These sources appear more consistent with an interpretation of FR I/FR II short-lived progenitors, rather than an intrinsically different radio class as it emerges from the multi-band studies of LCC. Nevertheless, a large overlap between the FR 0 and LLC populations cannot be excluded.

\section{What Causes the Deficit of Extended Radio Emission in FR 0s?}

While VLBI observations [27] and forthcoming enhanced Multi-Element Radio Linked Interferometer Network (eMERLIN) [63] observations show a larger fraction of asymmetric pc-scale radio structures, in JVLA maps $[21,26]$, the jets appear more symmetric at larger scales. In addition, the nuclear luminosities of FR 0s reconcile with those of FR Is, suggesting similar AGN energetics. Hence, these results concur on the picture that FR 0 jets are probably launched relativistic at pc scale, while, at a larger scale, they decelerate showing smaller Lorentz factors $\Gamma_{j e t} 4$.

Assuming a stationary jet scenario, the idea of a jet stratification with different velocity patterns [64] seems to better reproduce the general properties of the FR 0 class. The similarity of the nuclear properties of FR Is and FR 0s suggests that, within a few parsecs, the jet physics of the two FR classes need to be analogous. Therefore, as valid for FR Is, we speculate that a inner relativistic spine is likely to be responsible to produce the multi-band properties of the unresolved radio core and observed pc-scale jet asymmetry. However, to account for the confined jet capabilities of the FR 0s, the inner spine should be limited in length along the jet $(\lesssim 1 \mathrm{kpc})$. The outer layer travelling with a mild relativistic speed $(\sim 0.3 c)$ will dominate on a kpc scale and will account for the observed extended jet morphologies. The mild relativistic jet bulk speed and a possible intrinsic spine weakness together with entrainment of the galaxy medium could concur to a possible loss of jet stability. In this scenario, the jets would suffer from deceleration and premature disruption before escaping the host core radius, slowly burrowing their way into the external medium. Nevertheless, the optical host magnitudes of the FR 0s, a proxy for local density, comparable with those of FR Is, do not concur with the idea of a dense galaxy-scale environment which could cause the jet frustration through the interaction with interstellar density $[65,66]$. Alternatively, the scarcity of sites of plasma accelerations along the jet could also account for their restrained jet capabilities.

In a time-dependent jet scenario, an intermittent nuclear activity injects sporadically relativistic plasma in the jet and hence does not provide sufficient bulk flow to sustain the jet along large distances from the $\mathrm{BH}$. This lack of plasma injection might reduce the jet momentum and cause significant jet deceleration within the galaxy. To infer the duty cycles of FR 0s by comparing the space densities of FR 0s with respect to the extended FR Is, short active phases of a few thousands years are strongly favoured with respect to longer ones [29]. Analogously to FR 0s, LLC have also been interpreted

4 The Lorentz factor for jets is $\Gamma_{j e t}=\frac{1}{\sqrt{1-\left(v_{j e t} / c\right)^{2}}}$ where $\mathrm{v}_{j e t}$ is the jet speed. 
as powered by a short-lived outburst of the central activity, which may turn in compact radio jets [61]. Deep Low-frequency radio observations with LOFAR could reveal past radio activities of FR 0s and measure their activity recurrence. Nevertheless, a preliminary analysis on a small number of sources in the LoTSS survey seems to reject this hypothesis.

A further scenario proposes that FR 0s have low, prograde $\mathrm{BH}$ spin, which would limit the extracting energy to launch the jet and eventually evolve into full-fledged FR Is thanks to the increasing angular momentum of the accreting material [59]. Assuming a $\mathrm{BH}$ spin- $\Gamma_{j e t}$ dependence (e.g., $[67,68])$, the low BH spin would be the ultimate reason for the lower bulk Lorentz factor, reducing the ability of the FR $0 s^{\prime}$ jets to penetrate the galaxy medium. The low $\mathrm{BH}$ spin could be caused by a possibly poorer large-scale environment than that of FR Is [69], which sets (i) a less intense accretion history than FR Is, or (ii) a less frequent merger history [70] to favor BH-BH coalescence, which spins up the central $\mathrm{BH}$, a condition required to produce extended relativistic jets.

\section{High Energy (Gev-Tev) View of FR 0s}

While the previous $\gamma$-ray mission, EGRET, detected a few RGs (e.g., [71,72]), the Fermi $\gamma$-ray space telescope [73] has revolutionised our knowledge of the sky above $100 \mathrm{MeV}$. Among the outstanding results reached in ten years of survey, there is certainly the discovery of RGs as an whole class of GeV emitters [74]. Contrarily to blazars, where relativistic effects boost and blue-shift the emission of the jet that is closely aligned to the observer's line of sight, in RGs, where the viewing angle of the jet is large $\left(>1 / \Gamma_{j e t}\right)$, the detection of such sources at high energies is disfavoured. However, misaligned RGs were also detected by Fermi-LAT in only 15 months of survey [74] and their number continues to increase [75,76]. A few of them are also known as VHE emitters including M87 ( $\sim 16 \mathrm{Mpc})$, the first extra-galactic source detected at VHE energies, and Centaurus A (CenA), the nearest ( $\sim 4$ Mpc) AGN to $\mathrm{us}^{5}$. Although misaligned RGs represent $2 \%$ of the entire extra-galactic $\gamma$-ray population that is dominated by blazars $[77,78]$, they are extremely relevant in addressing problems related to the jet structure, the particle acceleration mechanism and the location of the acceleration sites. Moreover, RGs have been known to significantly contribute to the extra-galactic diffuse $\gamma$-ray background $[79,80]$ and, interestingly, they are often invoked as possible sources of high-energy neutrinos and ultra-high energy cosmic-rays [81,82].

Being an emerging class of low-luminosity RGs, so far, only a few studies have focused on FR 0s in the $\mathrm{GeV}$ domain. Currently, only one FR 0 has been associated with a $\gamma$-ray counterpart by Fermi, i.e., Tol 1326-379 [28]. The source is characterized by a GeV luminosity $\left(\mathrm{L}_{>1 \mathrm{GeV}}=2 \times 10^{42} \mathrm{erg} \mathrm{s}^{-1}\right)$ lower than blazars, consistent with FR Is but with a slightly steeper photon index $\left(\Gamma_{\gamma}=2.8\right)$ than classical FR Is. The radio-GeV spectral energy distribution (SED) of the core is double-humped (see Figure 4a) similar to other jet-dominated radio-loud AGN. Despite the similar radio luminosity, M 87 is less luminous than Tol 1326-379 by a factor of 30 at $1 \mathrm{GeV}$ and has a flatter SED in the $\gamma$-ray domain. In contrast, the SEDs of CenA and Tol 1326-379 are quite similar in shape with a steep $\gamma$-ray trend, but the former source is about two orders of magnitude fainter.

At low energies, the non-thermal synchrotron component of Tol 1326-379 dominates over any accretion-related contribution, further supporting the case of an ADAF disc suggested by the X-ray study (see [30] and Section 2.1). The peak at the high-energies (so-called Compton peak) appears to be relatively prominent, comparable with or even higher than the synchrotron one, differently from BL Lac sources. The whole SED can be consistently reproduced by a synchrotron and synchrotron-self-Compton (SSC) model assuming either an aligned $\left(\theta_{v}<1 / \Gamma_{j e t}\right)$ or a misaligned $\left(\theta_{v} \sim 30^{\circ}\right.$ [83]) jet, with a total jet energy flux of the order of few $10^{44} \mathrm{erg} \mathrm{s}^{-1}$ [83].

5 In addition to M 87 and CenA, there are other four radio galaxies detected at TeV energies, i.e., NGC 1275 (d $~ 76.7 \mathrm{Mpc})$, IC $310(\mathrm{~d} \sim 82.8 \mathrm{Mpc})$, PKS 0625-35 (d $\sim 245 \mathrm{Mpc})$ and 3C $264(\mathrm{~d} \sim 95.1 \mathrm{Mpc})$. 
Is Tol 1326-379 a unique $\gamma$-ray case? It has been estimated that core-dominated RGs nearby, i.e., FR0s and Core Galaxies [31], can account for $\sim 4-18 \%$ of the unresolved $\gamma$-ray background below $50 \mathrm{GeV}$ observed by the LAT instrument on-board Fermi [84]. Unfortunately, no FR 0 is listed among the $\sim 37$ non-blazar AGN in the recently released Fourth LAT AGN Catalog (4LAC, [76]). Nevertheless, one should note that Tol 1326-379 was initially classified as a blazar and a careful analysis of its multi-wavelength properties revealed its FR 0 nature [28]. Furthermore, the 4LAC includes a large number ( 1090) of sources defined as blazar candidates of uncertain type (BCUs), which could hide new $\gamma$-ray FR 0s. The range of $\gamma$-ray fluxes and photon indexes of BCUs $\left(\Gamma_{\gamma}=1.5-3.0\right)$ are also compatible with those of Tol 1326-379, which can be taken as a reference for FR 0s at high energies. The cross-correlation of the BCUs from the 4LAC with the FR0CAT did not return any match. However, the FR0CAT selected only sources in the northern hemisphere, while more than $50 \%$ of the BCUs are in the less explored southern sky, where Tol 1326-379 itself has been found. Thus, future efforts must be devoted to extend the search for $\gamma$-ray FR 0 sources to this part of the sky (see [17]).

Interestingly, spectral fitting of the synchrotron peak of the SED of the BCUs in the 4LAC reveals an emerging population of faint, BL Lac-like sources characterized by a low synchrotron peak $\left(10^{13-14} \mathrm{~Hz}\right.$, [76]). A fraction of these sources could be truly faint low-power BL Lacs (rather than located at high redshift) and possibly represent the aligned counterparts of FR 0s. The joint study of the two classes can be an effective way to explore the jet formation and the emission processes at the lowest levels of accretion onto the supermassive $\mathrm{BH}$ (see [45]).

Finally, it is important to stress that the Fermi catalogues place a $5 \sigma$ threshold to the detection of a source, which might leave out inherently faint $\gamma$-ray emitters (see [85] for an example). Therefore, progress in the characterization of the FR 0 class in the $\gamma$-ray window requires ad hoc analysis of the individual targets, exploiting the whole Fermi dataset, the most up-to-date calibrations and data selection, to look for sub-threshold emission. Besides the likelihood analysis of individual sources, the $\gamma$-ray properties of FR 0s can also be studied collectively by means of techniques searching for a signal in excess over the background in stacked data of a large sample (see [86] as example). These procedures could unearth a non-negligible population of low-luminosity RGs and FR 0s emitting at HE [87].

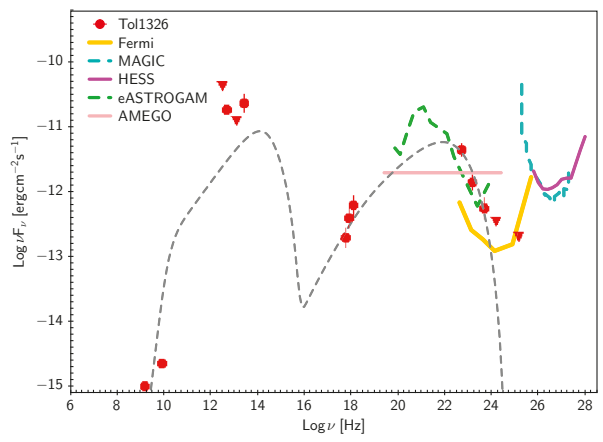

(a)

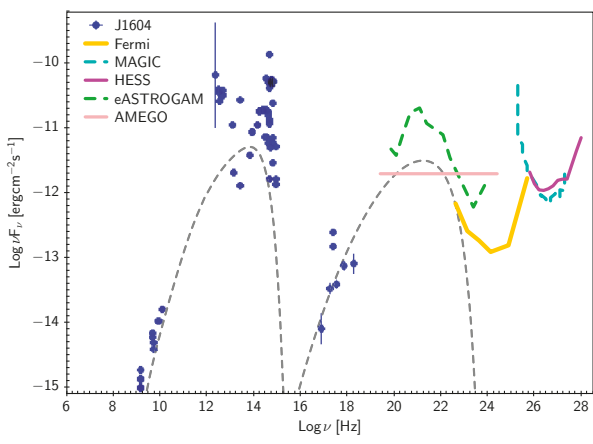

(b)

Figure 4. (a) the SED of Tol 1326-379 (red points), the only FR 0 observed by Fermi so far, is modelled with a one-zone synchrotron-SSC model (grey dashed line). Following the modelling presented in [83], a moderate viewing angle $\left(\sim 30^{\circ}\right)$ and bulk motion $\left(\Gamma_{\text {jet }} \sim 2\right)$ have been assumed. $(\mathbf{b})$ the SED of another FR 0, J160426.51 + 17 (blue points) with a model similar to that applied to Tol 1326-379 is shown (data from [28]). The expected sensitivity curves of current and future instruments in the MeV to $\mathrm{TeV}$ window are plotted (see the legend). While, in the framework of standard, one-zone leptonic models, a detection in the VHE range seems unlikely, both sources could be detected at MeV energies by future missions such as AMEGO [88] and e-ASTROGAM [89] (see Section 4.1). 


\subsection{Perspectives with Upcoming MeV-TeV Observatories}

Here, we test whether FR 0s could be detected by current and future generations of HE and VHE telescopes. We use two FR 0s as reference (Figure 4): while one is Tol 1326-379, detected by Fermi, the other source is a nearby $(z=0.04)$ FR 0 radio galaxy, J160426.51+17. It shows a flat X-ray spectral index $\left(\Gamma_{X}=1.1 \pm 0.3\right)$ and X-ray luminosity similar to Tol 1326-379 (see [30]) that make it a potential candidate for a $\gamma$-ray detection. As an exercise, we adjusted the model adopted for Tol 1326-379 to reproduce the SED of J160426.51+17 (Figure 4b): the output in the GeV window could be just at the Fermi detection limit.

Of equal importance for the characterization of the HE output of these sources will be the synergies between the VHE and the rest of the HE window. In particular, the Fermi observations have shown that, in RGs, the $0.1-100 \mathrm{GeV}$ band probes the Compton component beyond the peak, i.e., the decaying tail, while the peak likely falls in the MeV energy range. Therefore, missions such as AMEGO [88] and $e$-ASTROGAM [89], proposed with the goal of exploring the $\mathrm{MeV}$ sky, will be crucial to make progress in our knowledge of the processes producing the most energetic radiation. As an example, in Figure 4, we plotted the expected sensitivity curves for the two instruments on the SEDs of Tol 1326-379 and J160426.51+17. In both cases, the two telescopes would detect the two FR 0s at $\mathrm{MeV}$ energies. By filling the gap between the $\mathrm{X}$-ray and $\mathrm{GeV}-\mathrm{TeV}$ band, $\mathrm{MeV}$ measurements will also play an important role to discriminate between leptonic and hadronic models (see, e.g., [90]).

In a $\mathrm{TeV}$ regime, we compare the flux sensitivity curves of current Cherenkov telescopes (MAGIC [91], VERITAS [92], H.E.S.S. [93]) with the SEDs of the two FR 0s cases (Figure 4). The Inverse Compton bump is modelled with a power-law component [28] that fits the measured points in the Fermi band; above $10^{24} \mathrm{~Hz}$ the source is undetected. The predicted SED in the VHE regime is well below the sensitivity of all the Cherenkov telescopes in $50 \mathrm{~h}$ of observation (see Figure 5 for a zoom of the SED of Tol 1326-379 at VHE). A major step further will be possible thanks to the forthcoming Cherenkov Telescope Array (CTA) [94]. Thanks to the improvement in sensitivity by a factor 5-20 (depending on the energy range $10 \mathrm{GeV}-300 \mathrm{TeV}$ ) with respect to current Cherenkov telescopes, CTA will enable population studies and allow us to verify whether or not FR 0s are VHE emitters. Moreover, CTA will work as an observatory in synergy with other HE facilities, providing simultaneous data from a few $\mathrm{MeV}$ up to hundreds of TeV. This will guarantee the opportunity to test different mechanisms of VHE photon production in RGs in general $[95,96]$ and FR 0s in particular, as outlined in the next section.

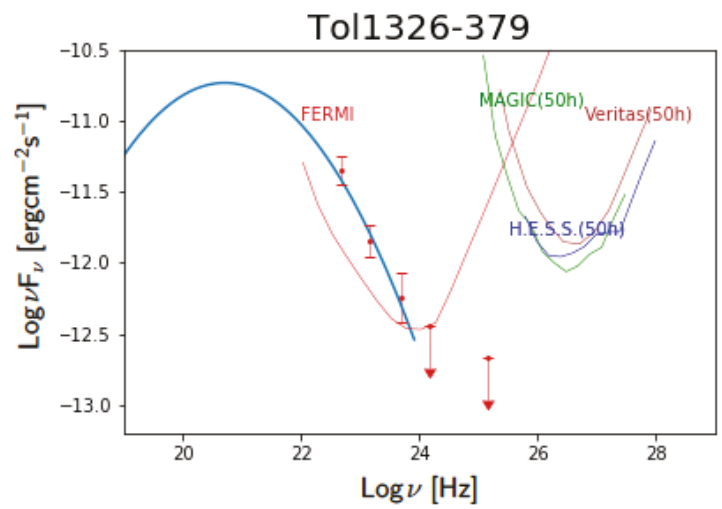

Figure 5. SED of Tol 1326-379 in the high energy band. The red points are Fermi-LAT measurements (with the corresponding sensitivity curve) fitted by a power-law component that reproduces the inverse Compton bump. We plot the expected differential sensitivity curves for $50 \mathrm{~h}$ of exposure of MAGIC (in green) [91], VERITAS (in brown) [92] and H.E.S.S telescopes (in blue) [93]. 


\section{Are FR 0s VHE Candidate Sources?}

The lack of evident FR 0s in the Fermi/LAT catalogues and in the TeV catalogues (e.g., TevCat and $\mathrm{TeGeV}$ catalogues $^{6}$ ) is not surprising. However, it is worth noting that the current Imaging Atmospheric Cherenkov Telescopes (IACT) operate in pointing mode, generally triggered by external alerts (e.g., the observation of an optical, $\mathrm{X}$-ray or $\gamma$-ray flare). Consequently, their $\mathrm{TeV}$ catalogues might include a bias towards blazars.

The question is therefore whether these sources may or may not be considered candidate $\mathrm{TeV}$ emitters, and which scenarios would imply their detection. In the case of Tol 1326-379, a detection $>100 \mathrm{GeV}$ would indicate the presence of a distinct radiative component, with respect to the SSC one, which should remain sub-dominant in the $0.1-10 \mathrm{GeV}$ band. Thus far, indications that such components could actually exist have been only found in the SED of the core of the radio galaxy Centaurus A [97,98]. Interestingly, its emission deviates from the simple extrapolation of the $<3 \mathrm{GeV}$ spectrum observed by Fermi, displaying a clear hardening at $<250 \mathrm{GeV}$ ([99] and references therein). A comprehensive discussion concerning the processes that could explain the origin of a $\mathrm{TeV}$ component is presented in [96]. Here, we review the different models of VHE photon production in AGN in relation to the FR 0 class.

The conventional leptonic jet (one-zone) SSC models predicts that X-ray-to- $\gamma$-ray part of the SED originates from inverse Compton (IC) scattering of low energy photons by relativistic electrons in a single emitting region within the inner jet. The scattered photons may have an internal origin, produced by the synchrotron mechanism (SSC [100]), or external origin, i.e., seed photons coming from the accretion disc, the broad line region, or the dusty torus (external Compton, EC, e.g., [101,102]). Still in the framework of leptonic models, a jet with a stratified kinematic structure opens to multi-zone emission models. In its simplest realisation, this could be a two-zone model with a inner fast spine $\left(\Gamma_{1} \gg 1\right)$ nested in a outer slower sheath $\left(\Gamma_{2}<\Gamma_{1}\right)$ [64]. Depending on the observer's line of sight, the emission of one of the two zones prevails, with the spine dominating at small angles (i.e., in blazars) and the layer at larger angles (in misaligned AGN). The two emitting regions may radiatively interact, by providing each other additional target synchrotron photons to be Compton scattered. Because of the velocity gradient between the two emitting regions, the synchrotron emission coming from one region is boosted in the other, making this an efficient way to produce HE emission.

Hadronic models, in which relativistic protons within the jet are ultimately responsible for the observed emission, have been also proposed (e.g., [103,104]). These relativistic protons can interact with the soft synchrotron radiation and produce electromagnetic cascades above several tens of $\mathrm{TeV}$ and be responsible for the neutrino production. FR 0 s have been recently proposed as cosmic neutrino emitters because of their $\gamma$-ray emission $[83,105]$.

In the $\mathrm{BH}$ proximity, the $\mathrm{BH}$ gap model predicts that particles streaming along the magnetic field lines in the magnetosphere can be accelerated to very high energies due to the formation of (partially) unscreened electric field regions (gaps) $[106,107]$. The width of the gap $h$ and the BH mass set the maximum $\gamma$-ray luminosity that can be emitted with Compton peaks in the range $10-100 \mathrm{TeV}$. In this gap model (stationary or time-dependent), very low accretion rates, or the presence of a radiatively-inefficient disc, represent a necessary condition for the detectability of magnetospheric VHE emission. This requires the accretion rate to be below a critical value, typically $\sim 0.01$ (e.g., [108]) and in turn leads to a constraint on the average jet power. Assuming a rapidly spinning $\mathrm{BH}$, the constraints on the gap size and accretion rate thus translate into a characteristic upper limit on the extractable VHE power of $L_{g a p}^{V H E} \propto 2 \times 10^{46} \dot{m}_{E} M_{B H}\left(h / r_{g}\right)$ [109] where $\dot{m}_{E}$ is the accretion rate scaled at the rate at the Eddington limit and $r_{g}$ is the gravitational radius. Assuming that the gap width in FR 0s is consistent with the gravitational radius as found from the VHE variability

6 TevCat (http://tevcat.uchicago.edu/) and TGeVCat (https://www.ssdc.asi.it/tgevcat/) are online catalogues for $\mathrm{TeV}$ astronomy. 
of M 87 (e.g., [110]) and assuming similar accretion rates to M 87 as supported by the results for CoreG [33] and for FR 0s [30], the expected VHE luminosities for FR 0s from the gap models are $10^{42-43} \mathrm{erg} \mathrm{s}^{-1}$. For M87, the VHE luminosity is 3-10 $\times 10^{40} \mathrm{erg} \mathrm{s}^{-1}$ (e.g., [111]), which is three orders below the expected VHE gap luminosity. Therefore, since FR 0s and M 87 have similar bolometric AGN power, we could expect TeV luminosities for the FR 0s to be an order of magnitude lower because of their on average one-magnitude lower $\mathrm{BH}$ masses $\left(\sim 10^{8} \mathrm{M}_{\odot}\right)$ than $\mathrm{M} 87$.

Relativistic jets are generally expected to be initially magnetically supported and models of collisionless magnetic reconnection are known to accelerate non-thermal electrons and produce HE photons by IC scattering (e.g., [112,113]) in the jets. Magnetic reconnection energy, when released, may provide an additional relativistic velocity component of the ejected plasma relative to the mean bulk flow of the jet, in any direction. This Doppler-boosted effect may lead to VHE emission even in misaligned AGN, including FR 0s. If the released magnetic power is responsible for the acceleration of the radiating relativistic particles, then the radio synchrotron radiation sets the minimum value to this power. Figure 6 compares the magnetic reconnection power driven by turbulence derived by [114] with the observed core radio luminosities of FR 0s taken from the FR0CAT [29]. We find that the magnetic reconnection power extracted from reconnection of the magnetic lines in the inner coronal region around the $\mathrm{BH}$ s of FR0s could be energetically sufficient to explain the core synchrotron radio emission from them, as also found valid for low-luminosity AGN [114,115]. Figure 6 also includes the $\gamma$-ray luminosity of Tol 1326-379, indicating that the magnetic reconnection produces enough power to emit $\mathrm{HE} \gamma$-ray radiation.

The scenario where $\gamma$-ray emission is produced by isotropically distributed relativistic electrons in the kpc-scale jet [116] has been raised to reconcile with the extended HE detection from the jet lobes of CenA [117] and with the possibility that X-ray jets could emit at VHE [118,119]. Relativistic electrons in the extended jets or lobes could Comptonise soft non-thermal X-ray radiation and CMB photons, which are beamed due to electron relativistic motion. The mild relativistic jet speed at a kpc scale expected for the FR 0s and their limited jet sizes disfavour but do not fully reject the possibility of VHE emission originated on a large jet scale from the FR 0 population.

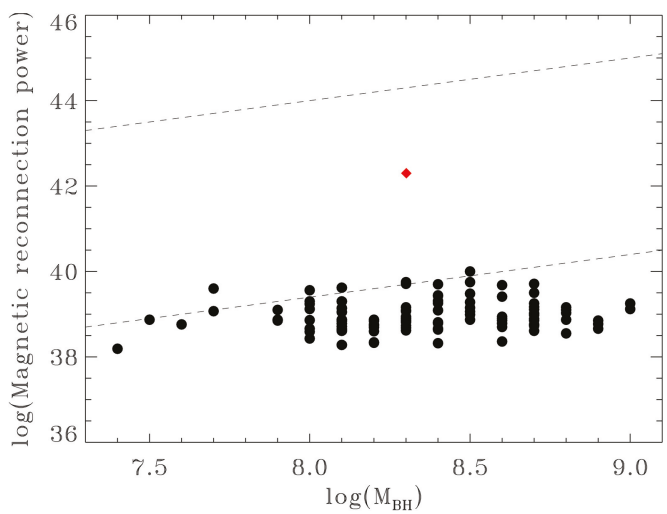

Figure 6. Turbulent driven magnetic reconnection power $\left(\mathrm{erg} \mathrm{s}^{-1}\right)$ against $\mathrm{BH}$ masses $\left(\mathrm{M}_{\odot}\right)$ compared to the observed radio emission from FIRST catalogue for the 104 FR 0s from the FR0CAT (black points). For Tol 1326-379, we also plot the high-energy ( $>1 \mathrm{GeV}$ ) luminosity (red diamond). The region enclosed between the two dashed lines represents the maximum range of magnetic reconnection power predicted for RGs for supermassive BHs with different internal conditions (see [114]).

On the whole, the VHE-emission models predict that FR 0s are TeV emitters with luminosities comparable or within an order of magnitude less than those observed from the core of local FR Is (i.e., M 87 and CenA). Nevertheless, the low brightness and further distance of the FR 0 population than the known TeV-emitting misaligned RGs (at least a factor of 2 in distance) hampers their detection 
at VHE even with future sensitive instruments. All the more, because it is unexpected, the detection of even a single FR 0 would open up interesting scenarios on the jet physics (radiative processes and jet accelerations) in these peculiar RGs.

\section{Summary, Conclusions and Future Perspectives}

The ongoing progress over the last few decades with consecutive Fermi catalogues and the upcoming advent of high-sensitivity $\mathrm{MeV}-\mathrm{TeV}$ facilities make the $\mathrm{HE}$ astronomy accessible to a large part of the community and these observatories play a unique scientific role in the comprehension of the jet physics in radio-loud AGN. In such a framework, FR 0s, compact RGs with a substantial deficit of extended radio emission [22], are catching the attention of the community because of their large abundance with respect to the other FR classes and their potentiality of being HE emitters as a new class.

In this work, we reviewed the multi-band properties of this class of RGs, from radio to X-rays. Then, based on the unique Fermi detection of the FR 0 population (i.e., Tol 1326-379 [28]) so far, we explored the possibility of $\mathrm{GeV}-\mathrm{TeV}$ emission of this abundant class of RGs, considering current (conventional and more exotic) models for $\gamma$-ray emission in AGN. Indeed, the Fermi detection of an FR 0 has opened up several scenarios to interpret its multi-wavelength SED. Whereas the similar broadband spectral nuclear properties of FR 0s and FR Is suggest common jet physics in the proximity to the $\mathrm{BH}$, the indisputable differences of the jet structures at large scales between the two classes might imply intrinsic variations in the particle accelerations and in HE emitting processes. A weak/short fast spine and the scarcity of sites of particle accelerations, limited to several pc along the jet, reconcile with the global multiband properties of FR 0s, the confined jet structures and the kpc-scale jet symmetry. In a time-dependent scenario, a nuclear recurrent activity could lead to a discontinuous plasma injection in the jet and its possible deceleration. In the framework of HE emitting sites, the core region appears to be most plausible scene where $\gamma$-ray radiation can be produced because of the dominant role of the fast spine in the jet, which can relativistically accelerate particles.

Several mechanisms can be proposed to address the particle acceleration in the inner part of the FR0 jets and their $\gamma$-ray production. Relativistic shocks between jet layers moving at different speeds are responsible for accelerating particles along the shock front and could produce significant $\gamma$-ray emission by IC. A magnetospheric gap model followed by a radiatively inefficient accretion flow to avoid $\gamma \gamma$-pair production seems to reconcile with the expected HE luminosities of FR 0s. Magnetic reconnection, where magnetic energy is transferred to accelerating particles, predicts non-thermal synchrotron powers that are sufficient to justify the observed radio core luminosities of FR 0s.

Detection of new FR 0s at HE and VHE bands in the next future or stringent upper limits could help to set the jet parameter space of this peculiar class of RGs and constrain models on particle acceleration and HE emission processes. In this framework, to test whether FR 0s could be detected at $\mathrm{HE}$, we compare the broadband SEDs of two cases of FR 0s with the sensitivity curves of MeV-GeV satellites and $\mathrm{TeV}$ Cherenkov telescopes. At MeV energies, the possible detection of the FR 0s will cover the gap between the X-ray and the VHE emission, crucial for having a comprehensive view of the jet properties. Below a few $\mathrm{GeV}$, the detection of new FR 0s appears to be feasible with current and future $\gamma$-ray facilities, and more sources could possibly hide among the unknown blazar candidates in the Fermi catalogue. At VHE, the steep GeV spectrum and low brightness of Tol 1326-379 make a detection unlikely. However, our knowledge of this class at high energies is still very limited, hence it will be important to investigate the FR 0s with more sensitive observatories, such as CTA in the near future.

The FR 0 population, which outnumbers the FR Is, is expected to contribute up to $\sim 18 \%$ to the extra-galactic $\gamma$-ray background [84]. Recently, TeV-emitting candidates associated with low-power compact radio sources hosted in red ETGs have been selected by [120]. The authors predict that a few hundred of these candidates will be detectable by new generations of Cherenkov telescopes in $50 \mathrm{~h}$ 
in the entire extra-galactic sky in the local Universe. We speculate that these $\mathrm{TeV}$ emitters could be the parent aligned population of the FR 0 s .

The optimal already-tested synergy between current radio, X-ray and $\gamma$-ray telescopes in the last decade and the upcoming generations of high-sensitivity and high-resolution facilities in such bands (i.e, SKA, LOFAR, ngVLA, eROSITA, Athena, Lynx, CTA) will offer unique insights into jet physics and particle acceleration mechanisms in the vicinity of the $\mathrm{BH}$. These new observatories will probe the very low and high energy bands of the SEDs of the RGs which populate the low luminosity tail of the local radio AGN luminosity [14]. In this very low-power regime, an enormous population of compact radio sources, which generally includes all nearby giant elliptical galaxies [121-125] consistent with an FR 0 classification, could accelerate jets and potentially be $\gamma$-ray emitters. CTA, which will unearth such a population of low-power HE sources, will provide important progress in our understanding of the AGN phenomena, by providing robust constraints on the $\mathrm{BH}$-jet coupling at a low-luminosity regime.

Author Contributions: R.D.B. has led several reference works on FR 0s, developing Sections 1, 2.2, 3, 5 and 6, and coordinated this review. E.T. has led the work on the results presented in Section 2.1 and contributed to Section 4, G.M. and B.B. has led the discussion in Section 4. All the authors contributed to the discussion and writing of this work.

Funding: R.D.B. acknowledges the support of STFC under grant ST/R000638/1 and the Italian grant (Progetto di Ateneo/CSP 2016). E.T acknowledges the financial contribution from the agreement ASI-INAF n.2017-14-H.0.

Acknowledgments: We thank Alessandro Capetti and Paola Grandi for the discussions that have contributed to the ideas that we expose in this review, and for sharing the multi-band data for the SED of Tol 1326-379. We thank the referees for providing useful comments to the original manuscript and X.P. Cheng for giving us permission to add his figure into this publication. Part of this work is based on archival data, software or online services provided by the Space Science Data Center - ASI. This research has made used of TOPCAT ${ }^{7}$ [126] for the preparation and manipulation of the tabular data.

Conflicts of Interest: The authors declare no conflict of interest. The founders had no role in the design of the study; in the collection, analyses, or interpretation of data; in the writing of the manuscript, or in the decision to publish the results.

\section{References}

1. Rogstad, D.H.; Ekers, R.D. Radio sources and elliptical galaxies. Astrophys. J. 1969, 157, 481. [CrossRef]

2. Heeschen, D.S. Radio observations of E and SO galaxies. Astron. J. 1970, 75, 523-529. [CrossRef]

3. Ekers, R.D.; Ekers, J.A. A survey of elliptical galaxies at $6 \mathrm{~cm}$. Astron. Astrophys. 1973, 24, 247.

4. Sadler, E.M. Radio and optical observations of a complete sample of $\mathrm{E}$ and $\mathrm{SO}$ galaxies. III. A radio continuum survey at 2.7 and 5.0 GHz. Astron. J. 1984, 89, 53. [CrossRef]

5. Kellermann, K.I.; Pauliny-Toth, I.I.K. Compact radio sources. Annu. Rev. Astron. Astr. 1981, 19, $373-410$. [CrossRef]

6. Slee, O.B.; Sadler, E.M.; Reynolds, J.E.; Ekers, R.D. Parsec-scale radio cores in early-type galaxies. Mon. Not. R. Astron. Soc. 1994, 269, 928-946. [CrossRef]

7. Wrobel, J.M.; Heeschen, D.S. Radio continuum sources in nearby and bright E/S0 galaxies: Active nuclei versus star formation. Astron. J. 1991, 101, 148-169. [CrossRef]

8. Fanaroff, B.L.; Riley, J.M. The morphology of extragalactic radio sources of high and low luminosity. Mon. Not. R. Astron. Soc. 1974, 167, 31-36. [CrossRef]

9. Nyland, K.; Harwood, J.J.; Mukherjee, D.; Jagannathan, P.; Rujopakarn, W.; Emonts, B.; Alatalo, K.; Bicknell, G.V.; Davis, T.A.; Greene, J.E.; et al. Revolutionizing our understanding of AGN feedback and its importance to galaxy evolution in the era of the next generation very large array. Astrophys. J. 2018, 859, 23. [CrossRef]

7 http://www.star.bris.ac.uk/ mbt/topcat/ 
10. Abazajian, K.N.; Adelman-McCarthy, J.K.; Agüeros, M.A.; Allam, S.S.; Allende Prieto, C.; An, D.; Anderson, K.S.J.; Anderson, S.F.; Annis, J.; Bahcall, N.A.; et al. The seventh data release of the Sloan Digital Sky Survey. Astrophys. J. Suppl. Ser. 2009, 182, 543. [CrossRef]

11. Becker, R.H.; White, R.L.; Helfand, D.J. The FIRST Survey: Faint Images of the Radio Sky at Twenty Centimeters. Astrophys. J. 1995, 450, 559. [CrossRef]

12. Condon, J.J.; Cotton, W.D.; Greisen, E.W.; Yin, Q.F., Perley; R.A., Taylor, G.B.; Broderick, J.J. The NRAO VLA Sky Survey. Astrophys. J. 1998, 115, 1693. [CrossRef]

13. Best, P.N.; Kauffmann, G.; Heckman, T.M.; Ivezić, Ž. A sample of radio-loud active galactic nuclei in the Sloan Digital Sky Survey. Mon. Not. R. Astron. Soc. 2005, 362, 9-24. [CrossRef]

14. Best, P.N.; Heckman, T.M. On the fundamental dichotomy in the local radio-AGN population: Accretion, evolution and host galaxy properties. Mon. Not. R. Astron. Soc. 2012, 421, 1569-1582. [CrossRef]

15. Baldi, R.D.; Capetti, A. Radio and spectroscopic properties of miniature radio galaxies: Revealing the bulk of the radio-loud AGN population. Astron. Astrophys. 2009, 508, 603-614. [CrossRef]

16. Baldi, R.D.; Capetti, A. Spectro-photometric properties of the bulk of the radio-loud AGN population. Astron. Astrophys. 2010, 519, A48. [CrossRef]

17. Sadler, E.M.; Ekers, R.D.; Mahony, E.K.; Mauch, T.; Murphy, T. The local radio-galaxy population at $20 \mathrm{GHz}$. Mon. Not. R. Astron. Soc. 2014, 438, 796-824. [CrossRef]

18. Whittam, I.H.; Riley, J.M.; Green, D.A.; Jarvis, M.J. The faint source population at 15.7 GHz-III. A high-frequency study of HERGs and LERGs. Mon. Not. R. Astron. Soc. 2016, 462, 2122-2137. [CrossRef]

19. Miraghaei, H.; Best, P.N. The nuclear properties and extended morphologies of powerful radio galaxies: The roles of host galaxy and environment. Mon. Not. R. Astron. Soc. 2017, 466, 4346-4363. [CrossRef]

20. Ghisellini, G. Extragalactic relativistic jets. Am. Inst. Phys. Conf. Ser. 2011, 1381, 180-198.

21. Baldi, R.D.; Capetti, A.; Giovannini, G. Pilot study of the radio-emitting AGN population: The emerging new class of FR 0 radio-galaxies. Astron. Astrophys. 2015, 576, A38. [CrossRef]

22. Baldi, R.D.; Capetti, A.; Giovannini, G. The new class of FR 0 radio galaxies. Astron. Nachr. 2016, 337, 114-119. [CrossRef]

23. Spinrad, H.; Djorgovski, S.; Marr, J.; Aguilar, L. A third update of the status of the 3 CR sources: Further new redshifts and new identifications of distant galaxies. Publ. Astron. Soc. Pac. 1985, 97, 932. [CrossRef]

24. Burns, J.O.; Feigelson, E.D.; Schreier, E.J. The inner radio structure of Centaurus A: Clues to the origin of thejet X-ray emission. Astrophys. J. 1983 273, 128-153. [CrossRef]

25. Alexander, P.; Leahy, J.P. Ageing and speeds in a representative sample of 21 classical double radio sources. Mon. Not. R. Astron. Soc. 1987, 225, 1-26. [CrossRef]

26. Baldi, R.D.; Capetti, A.; Giovannini, G. High-resolution VLA observations of FR0 radio galaxies: The properties and nature of compact radio sources. Mon. Not. R. Astron. Soc. 2019, 482, 2294-2304. [CrossRef]

27. Cheng, X.-P.; An, T. Parsec-scale Radio Structure of 14 Fanaroff-Riley Type 0 Radio Galaxies. Astrophys. J. 2018, 863, 155. [CrossRef]

28. Grandi, P.; Capetti, A.; Baldi, R.D. Discovery of a Fanaroff-Riley type 0 radio galaxy emitting at $\gamma$-ray energies. Mon. Not. R. Astron. Soc. 2016, 457, 2-8. [CrossRef]

29. Baldi, R.D.; Capetti, A.; Massaro, F. FR0CAT: A FIRST catalog of FR 0 radio galaxies. Astron. Astrophys. 2018, 609, A1. [CrossRef]

30. Torresi, E.; Grandi, P.; Capetti, A.; Baldi, R.D.; Giovannini, G. X-ray study of a sample of FR0 radio galaxies: Unveiling the nature of the central engine. Mon. Not. R. Astron. Soc. 2018, 476, 5535-5547. [CrossRef]

31. Balmaverde, B.; Capetti, A. The host galaxy/AGN connection in nearby early-type galaxies. Is there a miniature radio-galaxy in every "core" galaxy? Astron. Astrophys. 2006, 447, 97-112. [CrossRef]

32. Balmaverde, B.; Capetti, A.; Grandi, P. The Chandra view of the 3C/FR I sample of low luminosity radio-galaxies. Astron. Astrophys. 2006, 451, 35-44. [CrossRef]

33. Balmaverde, B.; Baldi, R.D.; Capetti, A. The accretion mechanism in low-power radio galaxies. Astron. Astrophys. 2008, 486, 119-130. [CrossRef]

34. Capetti A.; Baldi R.D.; Brienza M.; Morganti R.; Giovannini G. The low-frequency properties of FR 0 radio galaxies. Astron. Astrophys. submitted.

35. Kellermann, K.I.; Sramek, R.; Schmidt, M.; Shaffer, D.B.; Green, R. VLA Observations of Objects in the Palomar Bright Quasar Survey. Astron. J. 1989, 98, 1195. [CrossRef] 
36. Fabbiano, G.; Kim, D.-W.; Trinchieri, G. An X-ray Catalog and Atlas of Galaxies. Astrophys. J. Suppl. Ser. 1992, 80, 531. [CrossRef]

37. Hardcastle, M.J.; Worrall, D.M. Radio, optical and X-ray nuclei in nearby 3CRR radio galaxies. Mon. Not. R. Astron. Soc. 2000, 314, 359. [CrossRef]

38. Hardcastle, M.J.; Evans, D.A.; Croston, J.H. The active nuclei of $\mathrm{z}<1.03 \mathrm{CRR}$ radio sources. Mon. Not. R. Astron. Soc. 2009, 396, 1929.

39. Narayan, R.; Yi, I. Advection-dominated Accretion: Self-Similarity and Bipolar Outflows. Astrophys. J. 1995, 444, 231. [CrossRef]

40. Shimwell, T.W.; Tasse, C.; Hardcastle, M.J.; Mechev, A.P.; Williams, W.L.; Best, P.N.; Röttgering, H.J.A.; Callingham, J.R.; Dijkema, T.J.; de Gasperin, F.; et al. The LOFAR Two-metre Sky Survey. II. First, data release. Astron. Astrophys. 2019, 622, A1. [CrossRef]

41. Hardcastle, M.J.; Williams, W.L.; Best, P.N.; Croston, J.H.; Duncan, K.J.; Röttgering, H.J.A.; Sabater, J.; Shimwell, T.W.; Tasse, C.; Callingham, J.R.; et al. Radio-loud AGN in the first LoTSS data release. The lifetimes and environmental impact of jet-driven sources. Astron. Astrophys. 2019, 622, A12. [CrossRef]

42. Sabater, J.; Best, P.N.; Hardcastle, M.J.; Shimwell, T.W.; Tasse, C.; Williams, W.L.; Brüggen, M.; Cochrane, R.K.; Croston, J.H.; de Gasperin, F.; et al. The LoTSS view of radio AGN in the local Universe. The most massive galaxies are always switched on. Astron. Astrophys. 2019, 622, A17. [CrossRef]

43. Mingo, B.; Croston, J.H.; Hardcastle, M.J.; Best, P.N.; Duncan, K.J.; Morganti, R.; Rottgering, H.J.A.; Sabater, J.; Shimwell, T.W.; Williams, W.L.; et al. Revisiting the Fanaroff-Riley dichotomy and radio-galaxy morphology with the LOFAR Two-Metre Sky Survey (LoTSS). Mon. Not. R. Astron. Soc. 2019, 488, 2701. [CrossRef]

44. Buttiglione, S.; Capetti, A.; Celotti, A.; Axon, D.J.; Chiaberge, M.; Macchetto, F.D.; Sparks, W.B. An optical spectroscopic survey of the 3 CR sample of radio galaxies with $\mathrm{z}<0.3$. II. Spectroscopic classes and accretion modes in radio-loud AGN. Astron. Astrophys. 2010, 509, A6.

45. Capetti, A.; Raiteri, C.M. Looking for the least luminous BL Lacertae objects. Astron. Astrophys. 2015, 580, A73. [CrossRef]

46. Venturi, T.; Castaldini, C.; Cotton, W.D.; Feretti, L.; Giovannini, G.; Lara, L.; Marcaide, J.M.; Wehrle, A.E. VLBI Observations of a Complete Sample of Radio Galaxies. VI. The Two FR I Radio Galaxies B2 $0836+29$ and 3C 465. Astrophys. J. 1995, 454, 735. [CrossRef]

47. Giovannini, G.; Cotton, W.D.; Feretti, L.; Lara, L.; Venturi, T. VLBI Observations of a Complete Sample of Radio Galaxies: 10 Years Later. Astrophys. J. 2010, 552, 508. [CrossRef]

48. Kharb, P.; O’Dea, C.P.; Tilak, A.; Baum, S.A.; Haynes, E.; Noel-Storr, J.; Fallon, C.; Christiansen, K. VLBA and Chandra Observations of Jets in FR I Radio Galaxies: Constraints on Jet Evolution. Astrophys. J. 2012, 754, 1. [CrossRef]

49. O'Dea, C.P.; Baum, S.A.; Stanghellini, C. What Are the Gigahertz Peaked-Spectrum Radio Sources? Astrophys. J. 1991, 380, 66.

50. O'Dea, C.P. The Compact Steep-Spectrum and Gigahertz Peaked-Spectrum Radio Sources. Publ. Astron. Soc. Pac. 1998, 110, 493. [CrossRef]

51. Guainazzi, M.; Siemiginowska, A.; Stanghellini, C.; Grandi, P.; Piconcelli, E.; Azubike Ugwoke, C. A hard $\mathrm{X}$-ray view of giga-hertz peaked spectrum radio galaxies .Astron. Astrophys. 2006, 446, 87. [CrossRef]

52. Labiano, A. Tracing jet-ISM interaction in young AGN: Correlations between [O III] $\lambda 5007 \AA$, and 5-GHz emission. Astron. Astrophys. 2008, 488, L59. [CrossRef]

53. Migliori, G.; Siemiginowska, A.; Kelly, B.C.; Stawarz, Ł.; Celotti, A.; Begelman, M.C. Jet Emission in Young Radio Sources: A Fermi Large Area Telescope Gamma-Ray View. Astrophys. J. 2014, 780, 165. [CrossRef]

54. Siemiginowska, A.; Sobolewska, M.; Guainazzi, M.; Hardcastle, M.; Migliori, G.; Ostorero, L.; Stawarz, L. X-ray Properties of the Gigahertz Peaked and Compact Steep Spectrum Sources. Astrophys. J. 2008, 684, 811. [CrossRef]

55. Tengstrand, O.; Guainazzi, M.; Siemiginowska, A.; Fonseca Bonilla, N.; Labiano, A.; Worrall, D.M.; Grandi, P.; Piconcelli, E. The X-ray view of giga-hertz peaked spectrum radio galaxies. Astron. Astrophys. 2009, 501, 89. [CrossRef]

56. Vink, J.; Snellen, I.; Mack, K.-H.; Schilizzi, R. The X-ray properties of young radio-loud AGN. Mon. Not. R. Astron. Soc. 2006, 367, 928. [CrossRef]

57. Morganti, R.; Oosterloo, T. The interstellar and circumnuclear medium of active nuclei traced by H I $21 \mathrm{~cm}$ absorption. Astron. Astrophys. Rev. 2018, 26, 4. [CrossRef] 
58. Urry, C.M.; Padovani, P. Unified schemes for radio-loud active galactic nuclei. Publ. Astron. Soc. Pac. 1995, 107, 803-845. [CrossRef]

59. Garofalo, D.; Singh, C.B. FR 0 Radio Galaxies and Their Place in the Radio Morphology Classification. Astrophys. J. 2019, 871, 259. [CrossRef]

60. Kunert-Bajraszewska, M.; Thomasson, P. A survey of Low Luminosity Compact sources. Astron. Nachr. 2009, 330, 210. [CrossRef]

61. Kunert-Bajraszewska, M.; Gawroński, M.P.; Labiano, A.; Siemiginowska, A. A survey of low-luminosity compact sources and its implication for the evolution of radio-loud active galactic nuclei-I. Radio data. Mon. Not. R. Astron. Soc. 2010, 408, 2261. [CrossRef]

62. Kunert-Bajraszewska, M.; Labiano, A.; Siemiginowska, A.; Guainazzi, M. First, X-ray observations of low-power compact steep spectrum sources. Mon. Not. R. Astron. Soc. 2014, 437, 3063. [CrossRef]

63. Baldi, R.D.; Capetti, A.; Giovannini, G. The eMERLIN view of FR 0 radio galaxies. In preparation.

64. Ghisellini, G.; Tavecchio, F.; Chiaberge, M. Structured jets in TeV BL Lac objects and radiogalaxies. Implications for the observed properties. Astron. Astrophys. 2005, 432, 401. [CrossRef]

65. Ledlow, M.J.; Owen, F.N. 20 cm VLA Survey of Abell Clusters of Galaxies. VI. Radio/Optical Luminosity Functions. Astrophys. J. 1996, 112, 9. [CrossRef]

66. Kaiser, C.R.; Best, P.N. Luminosity function, sizes and FR dichotomy of radio-loud AGN. Mon. Not. R. Astron. Soc. 2007, 381, 1548. [CrossRef]

67. Tchekhovskoy, A.; Narayan, R.; McKinney, J.C. Black Hole Spin and The Radio Loud/Quiet Dichotomy of Active Galactic Nuclei. Astrophys. J. 2010, 711, 50. [CrossRef]

68. Maraschi, L.; Colpi, M.; Ghisellini, G.; Perego, A.; Tavecchio, F. On the role of black hole spin and accretion in powering relativistic jets in AGN. J. Phys. Conf. Ser. 2012, 355, 012016. [CrossRef]

69. Capetti A.; Massaro F.; Baldi R.D. The large-scale environment of FR 0 radio galaxies. Astron. Astrophys. 2019, submitted.

70. Dubois, Y.; Volonteri, M.; Silk, J. Black hole evolution-III. Statistical properties of mass growth and spin evolution using large-scale hydrodynamical cosmological simulations. Mon. Not. R. Astron. Soc. 2014, 440, 1590. [CrossRef]

71. Steinle, H.; Bennett, K.; Bloemen, H.; Collmar, W.; Diehl, R.; Hermsen, W.; Lichti, G.G.; Morris, D.; Schonfelder, V.; Strong, A.W.; et al. COMPTEL observations of Centaurus A at MeV energies in the years 1991 to 1995. Astron. Astrophys. 1998, 330, 97.

72. Mukherjee, R.; Halpern, J., Mirabal, N.; Gotthelf, E.V. Is the EGRET Source 3EG J1621+8203 the Radio Galaxy NGC 6251? Astrophys. J. 2002, 574, 693. [CrossRef]

73. Atwood, W.B.; Abdo, A.A.; Ackermann, M.; Althouse, W.; Anderson, B.; Axelsson, M.; Baldini, L.; Ballet, J.; Band, D.L.; Barbiellini, G.; et al. The Large Area Telescope on the Fermi Gamma-Ray Space Telescope Mission. Astrophys. J. 2009, 697, 1071. [CrossRef]

74. Abdo, A.A.; Ackermann, M.; Ajello, M.; Baldini, L.; Ballet, J.; Barbiellini, G.; Bastieri, D.; Bechtol, K.; Bellazzini, R.; Berenji, B.; et al. Fermi Large Area Telescope Observations of Misaligned Active Galactic Nuclei. Astrophys. J. 2010, 720, 912. [CrossRef]

75. Sahakyan, N.; Baghmanyan, V.; Zargaryan, D. Fermi-LAT observation of nonblazar AGNs. Astron. Astrophys. 2018, 614, A6. [CrossRef]

76. The Fermi-LAT collaboration. Fermi Large Area Telescope Fourth Source Catalog. arXiv 2019, arXiv:1902.10045.

77. Ackermann, M.; Ajello, M.; Atwood, W.B.; Baldini, L.; Ballet, J.; Barbiellini, G.; Bastieri, D.; Becerra Gonzalez, J.; Bellazzini, R.; Bissaldi, E.; et al. The Third Catalog of Active Galactic Nuclei Detected by the Fermi Large Area Telescope. Astrophys. J. 2015, 810, 14. [CrossRef]

78. Acero, F.; Ackermann, M.; Ajello, M.; Albert, A.; Atwood, W.B.; Axelsson, M.; Baldini, L.; Ballet, J.; Barbiellini, G.; Bastieri, D.; et al. Fermi Large Area Telescope Third Source Catalog Astrophys. J. Suppl. Ser. 2015, 218, 23. [CrossRef]

79. Stawarz, Ł.; Kneiske, T.M.; Kataoka, J. Kiloparsec-Scale Jets in FR I Radio Galaxies and the $\gamma$-Ray Background. Astrophys. J. 2006, 637, 693. [CrossRef]

80. Di Mauro, M.; Manconi, S.; Zechlin, H.-S.; Ajello, M.; Charles, E.; Donato, F. Deriving the Contribution of Blazars to the Fermi-LAT Extragalactic $\gamma$-ray Background at $\mathrm{E}>10 \mathrm{GeV}$ with Efficiency Corrections and Photon Statistics. Astrophys. J. 2018, 856, 106. [CrossRef] 
81. Eichmann, B.; Rachen, J.P.; Merten, L.; van Vliet, A.; Becker Tjus, J. Ultra-high-energy cosmic rays from radio galaxies. J. Cosmol. Astropart. Phys. 2018, 2018, 036. [CrossRef]

82. Mannheim, K. High-energy neutrinos from extragalactic jets. Astropart. Phys. 1995, 3, 295. [CrossRef]

83. Tavecchio, F.; Righi, C.; Capetti, A.; Grandi, P.; Ghisellini, G. High-energy neutrinos from FR 0 radio galaxies? Mon. Not. R. Astron. Soc. 2018, 475, 5529. [CrossRef]

84. Stecker, F.W.; Shrader, C.R.; Malkan, M.A. The Extragalactic Gamma-Ray Background from Core Dominated Radio Galaxies. Astropart. Phys. 2019, 879, 68. [CrossRef]

85. Migliori, G.; Siemiginowska, A.; Sobolewska, M.; Loh, A.; Corbel, S.; Ostorero, L.; Stawarz, Ł. First, Detection in Gamma-Rays of a Young Radio Galaxy: Fermi-LAT Observations of the Compact Symmetric Object PKS 1718-649. Astrophys. J. Lett. 2016, 821, L31. [CrossRef]

86. Ackermann, M.; Ajello, M.; Anderson, B.; Atwood, W.B.; Axelsson, M.; Baldini, L.; Barbiellini, G.; Bastieri, D.; Bellazzini, R.; Bhat, P.N.; et al. Fermi LAT Stacking Analysis of Swift Localized GRBs Astrophys. J. 2016, 822, 68. [CrossRef]

87. Best, S.; Bazo, J. Search for gamma-ray counterparts of newly discovered radio astrophysical sources. arXiv 2019, arXiv:1906.01664.

88. Moiseev, A.; Amego Team. All-Sky Medium Energy Gamma-ray Observatory (AMEGO). In Proceedings of the 35th International Cosmic Ray Conference (ICRC2017), Busan, Korea, 10-20 July 2017; p. 798.

89. De Angelis, A.; Tatischeff, V.; Tavani, M.; Oberlack, U.; Grenier, I.; Hanlon, L.; Walter, R.; Argan, A.; von Ballmoos, P.; Bulgarelli, A.; et al. The e-ASTROGAM mission. Exploring the extreme Universe with gamma rays in the $\mathrm{MeV}-\mathrm{GeV}$ range. Exp. Astron. 2017, 44, 25. [CrossRef]

90. Petropoulou, M.; Dimitrakoudis, S.; Padovani, P.; Mastichiadis, A.; Resconi, E. Photohadronic origin of $\gamma$-ray BL Lac emission: Implications for IceCube neutrinos. Mon. Not. R. Astron. Soc. 2015, 448, 2412. [CrossRef]

91. Sitarek, J.; Carmona, E.; Colin, P.; Mazin, D.; Tescaro, D. Performance of the MAGIC telescopes after the major upgrade. In Proceedings of the 34th International Cosmic Ray Conference (ICRC2015), The Hague, The Netherlands, 30 July-6 August 2015; p. 981.

92. Park, N.; VERITAS Collaboration. Performance of the VERITAS experiment. In Proceedings of the 34th International Cosmic Ray Conference (ICRC2015), The Hague, The Netherlands, 30 July-6 August 2015; p. 771.

93. Holler, M.; de Naurois, M.; Zaborov, D.; Balzer, A.; Chalmé-Calvet, R. Photon Reconstruction for H.E.S.S. Using a Semi-Analytical Model. In Proceedings of the 34th International Cosmic Ray Conference (ICRC2015), The Hague, The Netherlands, 30 July-6 August 2015; p. 980.

94. Actis, M.; Agnetta, G.; Aharonian, F.; Akhperjanian, A.; Aleksić, J.; Aliu, E.; Allan, D.; Allekotte, I.; Antico, F.; Antonelli, L.A.; et al. Design concepts for the Cherenkov Telescope Array CTA: An advanced facility for ground-based high-energy gamma-ray astronomy. Exp. Astron. 2011, 32, 193. [CrossRef]

95. Angioni, R.; Grandi, P.; Torresi, E.; Vignali, C.; Knödlseder. Radio galaxies with the Cherenkov Telescope Array. Astropart. Phys. 2017, 92, 42. [CrossRef]

96. Rieger, F.; Levinson, A. Radio Galaxies at VHE Energies. Galaxies 2018 6, 116. [CrossRef]

97. Aharonian, F.; Akhperjanian, A.G.; Anton, G.; de Almeida, U.B.; Bazer-Bachi, A.R.; Becherini, Y.; Behera, B.; Benbow, W.; Bernlöhr, K.; Boisson, C.; et al. Discovery of Very High Energy $\gamma$-Ray Emission from Centaurus a with H.E.S.S. Astrophys. J. 2009, 695, L40. [CrossRef]

98. Sahakyan, N.; Yang, R.; Aharonian, F.A.; Rieger, F.M. Evidence for a Second Component in the High-energy Core Emission from Centaurus A? Astrophys. J. Lett. 2013, 770, L6. [CrossRef]

99. H. E. S. S. Collaboration; Abdalla, H.; Abramowski, A.; Aharonian, F.; Ait Benkhali, F.; Angüner, E.O.; Arakawa, M.; Armand, C.; Arrieta, M.; Backes, M.; et al. The $\gamma$-ray spectrum of the core of Centaurus A as observed with H.E.S.S. and Fermi-LAT. Astron. Astrophys. 2018, 619, A71.

100. Maraschi, L.; Ghisellini, G.; Celotti, A. A Jet Model for the Gamma-Ray-emitting Blazar 3C 279. Astrophys. J. Lett. 1992, 397, L5. [CrossRef]

101. Dermer, C.D. On the Beaming Statistics of Gamma-Ray Sources. Astrophys. J. 1995, 446, L63. [CrossRef]

102. Ghisellini, G.; Madau, P. On the origin of the gamma-ray emission in blazars. Mon. Not. R. Astron. Soc. 1996, 280, 67. [CrossRef]

103. Böttcher, M. Modeling the emission processes in blazars. Astrophys. Space Sci. 2007, 309, 95. 
104. Rani, B.; Petropoulou, M.; Zhang, H.; D'Ammando, F.; Finke, J.; Baring, M.; Boettcher, M.; Dimitrakoudis, S.; Gan, Z.; Giannios, D.; et al. Multi-Physics of AGN Jets in the Multi-Messenger Era. Bull. Am. Astron. Soc. 2019, 51, 92.

105. Jacobsen, I.B.; Wu, K.; On, A.Y.L.; Saxton, C.J. High-energy neutrino fluxes from AGN populations inferred from X-ray surveys. Mon. Not. R. Astron. Soc. 2015, 451, 3649. [CrossRef]

106. Ruderman, M.A.; Sutherland, P.G. Theory of pulsars: Polar gaps, sparks, and coherent microwave radiation. Astrophys. J. 1975, 196, 51. [CrossRef]

107. Gil, J.; Melikidze, G.I.; Geppert, U. Drifting subpulses and inner acceleration regions in radio pulsars. Astron. Astrophys. 2003, 407, 315. [CrossRef]

108. Yuan, F.; Narayan, R. On the Nature of X-ray-Bright, Optically Normal Galaxies. Astrophys. J. 2004, 612, 724. [CrossRef]

109. Katsoulakos, G.; Rieger, F.M. Magnetospheric Gamma-Ray Emission in Active Galactic Nuclei. Astrophys. J. 2018, 852, 112. [CrossRef]

110. Albert, J.; Aliu, E.; Anderhub, H.; Antonelli, L.A.; Antoranz, P.; Backes, M.; Baixeras, C.; Barrio, J.A.; Bartko, H.; Bastieri, D.; et al. Very High Energy Gamma-Ray Observations of Strong Flaring Activity in M 87 in 2008 February. Astrophys. J. Lett. 2008, 685, L23. [CrossRef]

111. Aharonian, F.; Akhperjanian, A.; Beilicke, M.; Bernlöhr, K.; Börst, H.-G.; Bojahr, H.; Bolz, O.; Coarasa, T.; Contreras, J.L.; Cortina, J.; et al. Is the giant radio galaxy M 87 a TeV gamma-ray emitter? Astron. Astrophys. 2003, 403, L1. [CrossRef]

112. Nalewajko K.; Giannios D.; Begelman M. C.; Uzdensky D. A.; Sikora M. Radiative properties of reconnection-powered minijets in blazars. Mon. Not. R. Astron. Soc. 2011, 413, 333. [CrossRef]

113. Cui, Y.-D.; Yuan, Y.-F.; Li, Y.-R.; Wang, J.-M. A General Relativistic External Compton-Scattering Model for TeV Emission from M 87. Astrophys. J. 2012, 746, 177. [CrossRef]

114. Kadowaki, L.H.S.; de Gouveia Dal Pino, E.M.; Singh, C.B. The Role of Fast Magnetic Reconnection on the Radio and Gamma-ray Emission from the Nuclear Regions of Microquasars and Low Luminosity AGNs. Astrophys. J. 2015, 802, 113. [CrossRef]

115. De Gouveia Dal Pino, E.M.; Piovezan, P.P.; Kadowaki, L.H.S. The role of magnetic reconnection on jet/accretion disk systems. Astron. Astrophys. 2010, 518, A5. [CrossRef]

116. Bednarek, W. GeV-TeV $\gamma$-rays produced by electrons in the kpc-scale jet as a result of Comptonization of the inner jet emission. Mon. Not. R. Astron. Soc. 2019, 483, 1003. [CrossRef]

117. Abdo, A.A.; Ackermann, M.; Ajello, M.; Atwood, W.B.; Baldini, L.; Ballet, J.; Barbiellini, G.; Bastieri, D.; Baughman, B.M.; Bechtol, K.; et al. Fermi Gamma-Ray Imaging of a Radio Galaxy. Science 2010, $328,725$. [PubMed]

118. Hardcastle, M.J.; Croston, J.H. Modelling TeV $\gamma$-ray emission from the kiloparsec-scale jets of Centaurus A and M87. Mon. Not. R. Astron. Soc. 2011, 415, 133. [CrossRef]

119. Liu, R.-Y.; Rieger, F.M.; Aharonian, F.A. Particle Acceleration in Mildly Relativistic Shearing Flows: The Interplay of Systematic and Stochastic Effects, and the Origin of the Extended High-energy Emission in AGN Jets. Astrophys. J. 2017, 842, 39. [CrossRef]

120. Balmaverde, B.; Caccianiga, A.; Della Ceca, R.; Wolter, A.; Belfiore, A.; Ballo, L.; Berton, M.; Gioia, I.; Maccacaro, T.; Sbarufatti, B. Te-REX: A sample of extragalactic TeV-emitting candidates. Astron. Astrophys. submitted.

121. Nagar, N.M.; Falcke, H.; Wilson, A.S.; Ho, L.C. Radio Sources in Low-Luminosity Active Galactic Nuclei. I. VLA Detections of Compact, Flat-Spectrum Cores. Astrophys. J. 2000, 542, 186. [CrossRef]

122. Falcke, H.; Nagar, N.M.; Wilson, A.S.; Ulvestad, J.S. Radio Sources in Low-Luminosity Active Galactic Nuclei. II. Very Long Baseline Interferometry Detections of Compact Radio Cores and Jets in a Sample of LINERs. Astrophys. J. 2000, 542, 197. [CrossRef]

123. Filho, M.E.; Barthel, P.D.; Ho, L.C. The Radio Properties of Composite LINER/H II Galaxies. Astrophys. J. Suppl. Ser. 2003, 142, 223. [CrossRef]

124. Nyland, K.; Young, L.M.; Wrobel, J.M.; Sarzi, M.; Morganti, R.; Alatalo, K.; Blitz, L.; Bournaud, F.; Bureau, M.; Cappellari, M.; et al. The ATLAS ${ }^{3 D}$ Project-XXXI. Nuclear radio emission in nearby early-type galaxies. Mon. Not. R. Astron. Soc. 2016, 458, 2221. [CrossRef] 
125. Baldi, R.D.; Williams, D.R.A.; McHardy, I.M.; Beswick, R.J.; Argo, M.K.; Dullo, B.T.; Knapen, J.H.; Brinks, E.; Muxlow, T.W.B.; Aalto, S.; et al. LeMMINGs-I. The eMERLIN legacy survey of nearby galaxies. 1.5-GHz parsec-scale radio structures and cores. Mon. Not. R. Astron. Soc. 2018, 476, 3478. [CrossRef]

126. Taylor, M. B. TOPCAT \& STIL: Starlink Table/VOTable Processing Software. Astronomical Data Analysis Software and Systems XIV. Publ. Astron. Soc. Pac. Conf. Series. 2005, 347, 29.

(C) 2019 by the authors. Licensee MDPI, Basel, Switzerland. This article is an open access article distributed under the terms and conditions of the Creative Commons Attribution (CC BY) license (http:/ / creativecommons.org/licenses/by/4.0/). 

MDPI

St. Alban-Anlage 66

4052 Basel

Switzerland

Tel. +41616837734

Fax +41 613028918

www.mdpi.com

Galaxies Editorial Office

E-mail: galaxies@mdpi.com www.mdpi.com/journal/galaxies

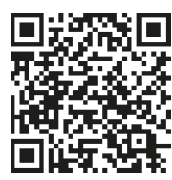



MDPI

St. Alban-Anlage 66

4052 Basel

Switzerland

Tel: +41 616837734

Fax: +41 613028918

www.mdpi.com 